Florida International University FIU Digital Commons

3-13-2007

\title{
Physical dynamic simulation of shipboard power system components in a distributed computational environment
}

Nagy Youssef Abed

Florida International University

DOI: $10.25148 /$ etd.FI13101509

Follow this and additional works at: https://digitalcommons.fiu.edu/etd

Part of the Electrical and Computer Engineering Commons

\section{Recommended Citation}

Abed, Nagy Youssef, "Physical dynamic simulation of shipboard power system components in a distributed computational environment" (2007). FIU Electronic Theses and Dissertations. 1100.

https://digitalcommons.fiu.edu/etd/1100 
Miami, Florida

PHYSICAL DYNAMIC SIMULATION OF SHIPBOARD POWER SYSTEM COMPONENTS IN A DISTRIBUTED COMPUTATIONAL ENVIRONMENT

A dissertation submitted in partial fulfillment of the

requirements for the degree of

DOCTOR OF PHILOSOPHY

in

ELECTRICAL ENGINEERING

by

Nagy Youssef Abed 
To: Dean Vish Prasad

College of Engineering and Computing

This dissertation, written by Nagy Youssef Abed, and entitled Physical Dynamic Simulation of Shipboard Power System Components in a Distributed Computational Environment, having been approved in respect to style and intellectual content, is referred to you for judgment.

We have read this dissertation and recommend that it be approved.

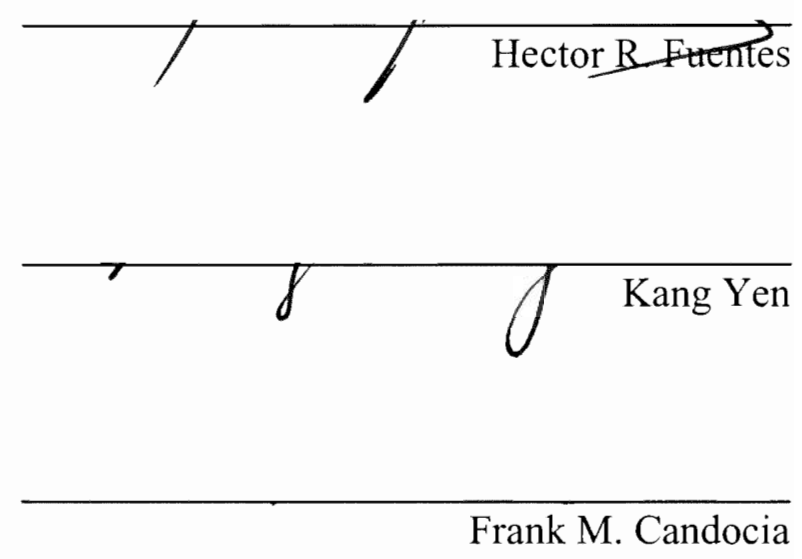

Osama Mohammed, Major Professor

Date of Defense: March 13, 2007

The dissertation of Nagy Youssef Abed is approved.

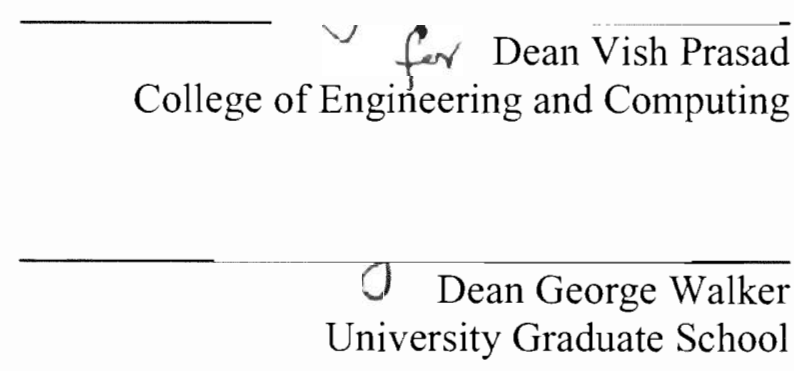

Florida International University, 2007 


\section{DEDICATION}

I dedicate this dissertation to my parents. Without their patience, understanding, support, and most of all love, the completion of this work would not have been possible. 


\section{ACKNOWLEDGMENTS}

I would like to express my sincere appreciation to my major professor and advisor,

Dr. Osama A. Mohammed, for his guidance, patience, support and research financial support he provided me throughout the course of this research. Without his aid and ideas, this dissertation would not be possible. I would like to thank my colleague Shreerang Ganu for his valuable help and discussion throughout the course of this dissertation. I would also like to thank Dr. Shuo Liu and Dr. Zhiqiang Liu for their helpful comments, help, and suggestions on the topics throughout the course of this research. I wish to thank the members of my dissertation committee for their valuable support, patience, and assistance.

Thanks are also due to all my friends who have helped me in various capacities in the completion phase of this dissertation. Amongst them is and Mr. Oscar Silveria and my colleague Nelson Cristaldo.

I offer my deepest gratitude to my family for their love, support and for their confidence in me. 
ABSTRACT OF THE DISSERTATION

PHYSICAL DYNAMIC SIMULATION OF SHIPBOARD POWER SYSTEM

COMPONENTS IN A DISTRIBUTED COMPUTATIONAL ENVIRONMENT

by

Nagy Youssef Abed

Florida International University, 2007

Miami, Florida

Professor Osama Mohammed, Major Professor

Shipboard power systems have different characteristics than the utility power systems. In the Shipboard power system it is crucial that the systems and equipment work at their peak performance levels.

One of the most demanding aspects for simulations of the Shipboard Power Systems is to connect the device under test to a real-time simulated dynamic equivalent and in an environment with actual hardware in the Loop (HIL). The real time simulations can be achieved by using multi-distributed modeling concept, in which the global system model is distributed over several processors through a communication link. The advantage of this approach is that it permits the gradual change from pure simulation to actual application.

In order to perform system studies in such an environment physical phase variable models of different components of the shipboard power system were developed using operational parameters obtained from finite element (FE) analysis.

These models were developed for two types of studies low and high frequency studies. Low frequency studies are used to examine the shipboard power systems 
behavior under load switching, and faults. High-frequency studies were used to predict abnormal conditions due to overvoltage, and components harmonic behavior.

Different experiments were conducted to validate the developed models. The Simulation and experiment results show excellent agreement.

The shipboard power systems components behavior under internal faults was investigated using FE analysis. This developed technique is very curial in the Shipboard power systems faults detection due to the lack of comprehensive fault test databases.

A wavelet based methodology for feature extraction of the shipboard power systems current signals was developed for harmonic and fault diagnosis studies.

This modeling methodology can be utilized to evaluate and predicate the NPS components future behavior in the design stage which will reduce the development cycles, cut overall cost, prevent failures, and test each subsystem exhaustively before integrating it into the system. 


\section{TABLE OF CONTENTS}

1. Introduction

1.1. Introduction

1.2. Phase variable motor model

1.3. Overview of simulation software for shipboard power system studies 8

1.3.1. Electromagnetic Transients Program (EMTP/ATP) 9

$\begin{array}{ll}\text { 1.3.2. PSPICE } & 10\end{array}$

1.3.3. Virtual Test Bed (VTB) 11

1.3.4. Matlab/Simulink 12

1.4. Classification of shipboard Power Distribution System studies $\quad 13$

1.5. Real Time simulations with Hardware in the Loop concept 14

$\begin{array}{ll}\text { 1.6. Literature review } & 15\end{array}$

1.6.1. Overview of the coupled field-circuit problems 16

1.6.2. Transformer modeling 18

1.6.3. Application of Wavelets in Power system 21

1.6.4. Integrated motor drive modeling 24

1.6.5. Modeling of Induction motor with internal faults 25

$\begin{array}{ll}\text { 1.7. Problem statement } & 27\end{array}$

1.8. Originality and significance $\quad 29$

1.9. Organization of dissertation 30

2. Coupled Field-Circuit Modeling 32

2.1. Introduction $\quad 32$

2.2. Finite element formulation 32

2.3. Electric circuit equations 36

2.4. Relation between magnetic and electric circuit equations 38

2.5. Coupling of field and circuit equations 38

2.6. Conductor representation in coupled-FE method 40

$\begin{array}{lll}\text { 2.6.1. Solid conductors } & 40\end{array}$

2.6.2. Stranded conductors $\quad 42$

2.6.3. Coupling with solid conductors 45

2.6.4. Coupling with stranded conductors $\quad 46$

2.7. Coupling of FE model with Simulink 47

2.8. Operational Parameters Extraction 48

2.8.1. Inductance calculation for high and low frequency studies $\quad 48$

2.8.2. Resistance calculation 51

2.8.3. Capacitance calculation $\quad 52$

3. Simulation of Shipboard Power System Components Using FEM 54

3.1. Introduction 54

3.2. Finite element modeling of the three phase power Transformer 54

3.2.1. The FEM model 54

3.2.2. Three phase transformer supplying linear load 55 
3.2.3. Three phase transformer supplying non-linear load 55

3.2.4. Three phase transformer inductances profile 63

3.2.5. Transformer parameters frequency dependence 75

3.3. Finite element modeling of the single phase transformer 75

3.3.1. The FEM model $\quad 75$

3.3.2. The Transformer inductances profile 76

3.4. Finite element modeling of three phase induction motor 78

3.4.1. The FE model description 78

3.4.2. Effect of supply harmonics on the induction machine 81

3.5. Finite element modeling of three phase cable 82

$\begin{array}{ll}\text { 3.5.1. The used Geometry } & 82\end{array}$

3.5.2. Low frequency parameters calculation $\quad 84$

3.5.3. High frequency parameters calculation 86

4. Simulation of Shipboard Power System Components under Abnormal Operating Conditions Using FEM 90

4.1. Introduction 90

4.2. Finite element modeling of the power transformer with internal $\begin{array}{ll}\text { faults } & 90\end{array}$

4.2.1. Transformer model with internal fault 90

4.2.2. Transformer model with arcing internal fault 93

4.2.3. Simulation results 95

4.3. Finite element model of the three phase induction machine with internal Fault $\quad 99$

4.3.1. Introduction $\quad 99$

4.3.2. The FE model 100

4.3.3. Simulation results 103

5. Modeling of shipboard Components for Low Frequency Studies 109

5.1. Introduction 109

5.2. Phase variable model of permanent magnet synchronous machine $\quad 109$

$\begin{array}{ll}\text { 5.2.1. Introduction } & 109\end{array}$

5.2.2. Details of the Phase variable model 111

5.2.3. Simulink implementation 114

5.2.4. Case studies 116

5.3. Cable modeling 118

5.3.1. The model 118

$\begin{array}{ll}\text { 5.3.2. Simulation results } & 120\end{array}$

$\begin{array}{ll}\text { 5.4. Transformer modeling } & 124\end{array}$

5.4.1. Introduction 124

5.4.2. Transformer phase variable model 125

$\begin{array}{ll}\text { 5.4.3. Simulations } & 127\end{array}$ 
6. Model Development for Shipboard Power System Components for High Frequency Studies

6.1. Introduction

6.2. Vector fitting

6.2.1. The theory of vector fitting

6.2.2. Pole identification

6.2.3. Finding the residues

6.2.4. Iteration

6.2.5. Synthesization by electrical network

6.3. Cable frequency dependent modeling

6.3.1. Cable frequency response

6.3.2. Fitting the cable frequency response

6.3.3. Simulations

6.4. Transformer high frequency modeling

6.4.1. Introduction

6.4.2. Inductance calculation

6.4.3. Resistance calculation

143

6.4.4. Capacitances calculation

6.4.5. Model reduction technique

6.4.6. Model description

6.4.7. Simulation results

7. Wavelets Transform 153

7.1. Introduction 153

7.2. Continuous Wavelet Transforms (CWT) 155

7.3. Choice of analyzing mother wavelet 156

7.4. Discrete Wavelet Transforms (DWT) 156

7.4.1. Multiresolution analysis 156

7.4.2. Waveform Effective Value Representation in DWT 159

7.5. Wavelet Packet Transform Algorithm (WPT) 160

$\begin{array}{lll}\text { 7.5.1. Theory } & 161\end{array}$

7.5.2. WPT Representation of the waveform indices 163

8. Classification And Characterization of Power System Events Using Wavelets

8.1. Introduction 165

8.2. Application of DWT as feature extractor for fault signals 165

8.2.1. Characterization of three phase transformers internal faults using DWT

8.2.2. Characterization of induction motor internal faults using DWT

8.3. Evaluation of transformer harmonic behavior using WPT 
9. Real-Time distributed Simulation of Power Electronic Systems and Drives 196 9.1. Introduction 196

9.2. System description 197

9.2.1. Drives Board 198

9.2.2. Block diagram and schematic interface 200

9.2.3. Distributed Computing Systems 201

9.2.4. Data Acquisition 202

9.2.5. Simulator configuration 202

10. Experimental Implementation 204

10.1. Introduction 204

10.2. Distributed simulation 204

10.3. DC Motor Drive simulation using HIL approach 207

10.4. Scalar based induction motor controller using HIL approach 210

10.5. Vector based induction motor controller using HIL approach 212

10.6. Evaluation of overvoltage in integrated motor drive using real time 217 distributed simulations

11. Recommendations for future work 220

12. Conclusion 221

$\begin{array}{ll}\text { References } & 223\end{array}$

$\begin{array}{ll}\text { Vita } & 243\end{array}$ 


\section{LIST OF TABLES}

TABLE

PAGE

3.1 The triangle conductors cable inductance matrix

3.2 The triangle conductors cable capacitance matrix

3.3 The circle conductors cable inductance Matrix

3.4 The circle conductors cable capacitance matrix

7.1 Frequency allocation in DWT analysis

8.1 WPT results for the DC side currents

8.2 WPT results for the transformer secondary current

8.3 WPT results for the transformer magnetizing current

10.1 Comparison of execution times for Simulink and distributed simulation 


\section{LIST OF FIGURES}

FIGURE

PAGE

1.1 Naval power distribution system general architecture

2.1 Schematic of the coupling between fem and external circuit connections

2.2 Schematic of the coupling between the FEM and Simulink

3.1 The used transformer geometry

3.2 Transformer core magnetizing curve

3.3 Transformer finite element mesh details

3.4 Transformer circuit domain representation

3.5 Transformer magnetizing current obtained from FE

3.6 Circuit model of the transformer, source and nonlinear load

3.7 Transformer secondary current for ideal voltage supply and voltage supply distorted by $3 \mathrm{rd}$, 5th, and 7th harmonics

3.8 Transformer secondary current for supply distorted by 9 th, 11 th, 60 13 th and 15 th harmonics

3.9 Transformer secondary current for different supply harmonics

3.10 DC side current for ideal supply and supply distorted by $3 \mathrm{rd}, 5$ th , and 7 th harmonics

3.11 DC side current for supply distorted by 9 th, 11th, 13th and 15th harmonics

3.12 Transformer phase a primary inductance as a function of the magnetizing current

3.13 Transformer mutual inductance between phase a primary and phase $b$ primary side as a function of the magnetizing current 
3.14 Transformer mutual inductance between phase a primary and phase $\mathrm{c}$ primary side as a function of the magnetizing current

3.15 Transformer mutual inductance between phase a primary and phase a secondary side as a function of the magnetizing current

3.16 Transformer mutual inductance between phase a primary and phase

$\mathrm{b}$ secondary side as a function of the magnetizing current

3.17 Transformer mutual inductance between phase a primary and phase c secondary side as a function of the magnetizing current

3.18 Transformer phase $b$ primary inductance as a function of the magnetizing current

3.19 Transformer mutual inductance between phase $b$ primary and phase c primary side as a function of the magnetizing current

3.20 Transformer mutual inductance between phase $b$ primary and phase a secondary side as a function of the magnetizing current

3.21 Transformer mutual inductance between phase b primary and phase $\mathrm{b}$ secondary side as a function of the magnetizing current

Transformer mutual Inductance between phase b primary and phase $\mathrm{c}$ secondary side as a function of the magnetizing current

3.23 Transformer phase c primary inductance as a function of the magnetizing current

3.24 Transformer mutual inductance between phase c primary and phase a secondary side as a function of the magnetizing current

3.25 Transformer mutual inductance between phase $\mathrm{c}$ primary and phase

$b$ secondary side as a function of the magnetizing current

3.26 Transformer mutual inductance between phase $\mathrm{c}$ primary and phase c secondary side as a function of the magnetizing current

3.27 Transformer phase a secondary inductance as a function of the magnetizing current

3.28 Transformer mutual inductance between phase a secondary and phase $b$ secondary side as a function of the magnetizing current 
3.29 Transformer mutual Inductance between phase a secondary and phase $\mathrm{c}$ secondary side as a function of the magnetizing current

3.30 Transformer phase b secondary inductance as a function of the 73 magnetizing current

3.31 Transformer mutual inductance between phase $b$ secondary and phase $\mathrm{c}$ secondary side as a function of the magnetizing current

3.32 Transformer phase $\mathrm{c}$ secondary inductance as a function of the magnetizing current

3.33 Transformer resistance frequency dependence

3.34 Transformer inductance frequency dependence

3.35 The $125 \mathrm{kVA}$ transformer geometry and the mesh details

3.36 The Transformer primary inductance as a function of the magnetizing current

3.37 Transformer mutual inductance between the primary and secondary as a function of the magnetizing current

3.38 Transformer secondary inductance as a function of the magnetizing current

3.39 Induction motor finite element mesh detail

3.40 Circuit domain model of the induction motor

3.41 Induction three phase motor starting currents

3.42 Induction motor phase a current for ideal supply and supply distorted by $3 \mathrm{rd}$, 5th, and 7 th harmonics

3.43 Induction motor torque profile for ideal supply and supply distorted by $3 \mathrm{rd}, 5$ th, and 7 th harmonics

3.44 The triangle conductors cable geometry

3.45 The circle conductors cable type Geometry and mesh details 
3.46 The triangle conductors cable magnetic flux density distribution @ $\mathrm{t}=10 \mathrm{msec}$

3.47 The circle conductors cable magnetic flux density distribution@ $\mathrm{t}=10 \mathrm{msec}$

3.48 The circle conductors cable mesh detail for the high frequency study

3.49 The triangle conductors cable current distribution at different 88 frequencies

3.50 The triangle conductors resistance and inductance variation with the supply frequency

3.49 The circle conductors cable current distribution at different frequencies

3.50 The circle conductors resistance and inductance variation with the frequency

4.1 FE domain and circuit domain representation of the transformer primary coils for (a) Turn to ground Fault (b) turn to turn Fault

4.2 Circuit model of the transformer with internal fault on its secondary side, source and nonlinear load

4.3 the arc equivalent circuit

4.4 Outline of coupling between FEM and the arc model

4.5 Transformer magnetic flux distribution for different cases on the secondary side

4.6 Transformer magnetic flux distribution for different cases on the primary side

4.7 Transformer primary currents and the DC side for different turn-toground faults

4.8 Transformer primary currents and the DC side for different turn-toground Faults

4.9 Transformer primary currents and the DC side for different turn-toturn Faults 
4.10 Transformer three phase primary, secondary, and DC currents for secondary turn-to -ground and turn-to-turn internal Faults

4.11 Finite element mesh of the induction motor

4.12 FE and circuit domain representation of the induction motor turn to 102 turn internal Fault

4.13 Circuit model of the Induction motor with stator internal fault

4.14 Magnetic flux distribution for normal Operation and for 12 shorted 103 turns with five broken bar under sinusoidal Source

4.15 The current distribution in the induction machine bars under broken 104 bars fault

4.16 Magnetic vector potential in the middle of the air-gap for different cases under sinusoidal supply

105

4.17 Magnetic vector potential in the air-gap for different internal faults under non-sinusoidal supply

4.18 Induction motor torque profile for different internal faults

106

4.19 Induction machine phase three phase currents for different internal 106 faults under sinusoidal Source

4.20 Induction machine three phase currents for different internal faults under sinusoidal Source

4.21 Induction machine phase a current for different internal faults under non-sinusoidal Source

108

5.1 Circuit element based representation of stator winding

5.2 Adjustable self- inductance

5.3 Inductance block of phase A

5.4 Controlled voltage source

5.5 The drive system simulation results 
$\begin{array}{lll}5.6 & \text { The lumped cable model } & 118\end{array}$

$\begin{array}{lll}5.7 & \text { The system used in the study } & 120\end{array}$

$\begin{array}{lll}5.8 & \text { The DC side current } & 120\end{array}$

$\begin{array}{lll}5.9 & \text { Cable phase a current waveform } & 121\end{array}$

$\begin{array}{lll}5.10 & \text { The Voltage drop across the cable } & 121\end{array}$

5.11 The motor model connected to a voltage source through the cable 122 model

5.12 The motor three phase currents

$\begin{array}{lll}5.13 & \text { The motor torque vs. time } & 123\end{array}$

$\begin{array}{lll}5.14 & \text { The motor speed vs. time } & 123\end{array}$

5.15 Simulink block flow implementation of the transformer model 126

$\begin{array}{lll}\text { 5.16 Transformer three phase magnetizing current } & 127\end{array}$

$\begin{array}{lll}\text { 6.1 Flowchart of HF model generation procedure } & 129\end{array}$

$\begin{array}{lll}6.2 & \text { Synthesization by electrical network } & 135\end{array}$

$\begin{array}{lll}6.3 & \text { The used cable geometry and the mesh details } & 136\end{array}$

$\begin{array}{lll}6.4 & \text { The frequency dependence of the cable resistance } & 137\end{array}$

$\begin{array}{lll}\text { 6.5 The frequency dependence of the cable inductance } & 137\end{array}$

$\begin{array}{lll}6.6 & \text { The magnitude six order approximation result and the error } & 138\end{array}$

6.7 The Phase angle approximation of the cable impedance 138

$\begin{array}{lll}6.8 & \text { The system used in the study } & 139\end{array}$

6.9 The inverter out voltage when connected to the developed HF cable 139 model

6.10 The inverter output voltage when connected to the normal lumped cable model 
6.11 The used Transformer Geometry

6.12 Lumped parameter equivalent of one winding

6.13 The Frequency dependence of the transformer inductance

6.14 The Frequency dependence of the transformer resistance

6.15 The reduction Technique

6.16 High frequency phase variable model of transformer

6.17 Primary current profile at different switching frequencies

6.18 Primary voltage profile at different switching frequencies

7.1 Time Frequency resolution of Wavelet Transform (WT)

7.2 Three decomposition levels with successive filtering and 158 downsampling by 2

7.3 WPT two decomposition levels with successive filtering and downsampling by 2

8.1 WTCs of phase c primary current for primary phase c 24 shorted turn-to-ground

8.2 WTCs of phase a primary current for primary phase c 24 shorted turn-to-ground

8.3 WTCs of phase b primary current for primary phase $\mathrm{c} 24$ shorted turn-to-ground

8.4 WTCs of the DC side current for primary phase c 24 shorted turnto-ground

8.5 WTCs of primary phase c current for turn-to-turn fault on phase $\mathrm{c}$

8.6 WTCs of primary phase b current for turn-to-turn fault on primary phase $\mathrm{c}$

8.7 WTCs of primary phase B current for turn-to-turn fault on primary phase $\mathrm{c}$ 
8.8 WTCs of the DC side current for turn-to-turn fault on primary phase c

8.9 WTCs of phase c primary current for arcing turn-to-ground fault on phase c primary

8.10 WTCs of phase a primary current for arcing turn-to-ground fault on phase c primary

8.11 WTCs of phase b primary current for arcing turn-to-ground fault on the primary of phase $\mathrm{c}$

8.12 WTCs of DC side current for arcing turn-to-ground fault on the primary of phase $\mathrm{C}$

8.13 WTCs of phase a primary current for arcing turn-to-turn fault the primary of phase $\mathrm{c}$

8.14 WTCs of phase b primary current for arcing turn-to-turn fault on 174 the primary of phase $\mathrm{c}$

8.15 WTCs of phase c primary current for arcing turn-to-turn fault on the primary of phase $\mathrm{c}$

8.16 WTCs of DC side current for arcing turn-to-turn fault on the primary of phase c

8.17 WTCs of phase a secondary current for arcing turn-to-turn fault on the primary of phase $\mathrm{c}$

8.18 WTCs of phase a secondary current for turn-to-ground fault on the secondary of phase $\mathrm{c}$

8.19 WTCs of phase b secondary current for turn-to-ground fault on the secondary of phase c

8.20 WTCs of phase c secondary current for turn-to-ground fault on the secondary of phase $\mathrm{c}$

8.21 WTCs of the de side current for turn-to-ground fault on the secondary of phase c

8.22 WTCs of Primary Phase a current for turn-to-ground fault on the secondary of phase $\mathrm{c}$ 
8.23 WTCs of Primary Phase b current for turn-to-ground fault on the secondary of phase $b$

8.24 WTCs of primary phase c current for turn-to-ground fault on the secondary of phase $\mathrm{c}$

8.25 WTCs of phase a secondary current for turn-to-turn fault on the secondary of phase c

8.26 WTCs of phase C secondary current for turn-to-turn fault on the secondary of phase $\mathrm{C}$

8-27 WTCs of phase b secondary current for turn-to-ground fault on the secondary of phase $\mathrm{c}$

8-28 WTCs of the de side current for turn-to-turn fault on the secondary of phase $\mathrm{c}$

8-29 WTCs of Primary Phase A current for phase c secondary turn-toturn fault

8-30 WTCs of primary phase b current for phase c secondary turn-to-turn fault

8-31 WTCs of phase a current for healthy motor under ideal voltage supply

8-32 WTCs of the induction motor phase a current for 44 shorted stator turns under ideal voltage supply

8-33 WTCs of phase a current for 4 broken bars under ideal voltage supply

8-34 WTCs of phase a current for 44 stator shorted turns with 4 broken bars under ideal voltage supply

8-35 The first 6 frequency bands for the DC side current under 3rd peaking harmonic distorted voltage supply

8-36 The first 6 frequency bands for the DC side current under 3rd flattened harmonic distorted voltage supply.

8-37 The first 6 frequency bands for the DC side current under 5th peaking harmonic distorted voltage supply 
8.38 The first 6 frequency bands for the DC side current under 5 th

flattened harmonic distorted voltage supply

8.39 The first 6 frequency bands for the DC side current under 9th peaking harmonic distorted voltage supply

8.40 The first 6 frequency bands for the DC side current under 9th flattened harmonic distorted voltage supply

8.41 The first 6 frequency bands for the DC side current under 11th peaking harmonic distorted voltage supply

8.42 The first 6 frequency bands for the DC side current under 11th flattened harmonic distorted voltage supply

9.1 The real-time simulator architecture

9.2 The drive board layout

9.3 Host-target real-time software architecture

10.1 Simulink model for PM synchronous vector control system

10.2 The final regrouped model

10.3 The motor voltage, current, speed and torque versus time

10.4 The Simulink block diagram for PMDC motor control

10.5 Block diagram of speed-current controller for an PMDC machine

10.6 The controller Simulink block diagram details

10.7 PMDC Machine speed, DC current, and duty versus time

10.8 Block diagram of a scalar controller for an induction motor

10.9 Inside the target PC Simulink block subsystem

10.10 Induction motor theta, rotation direction, theta and the angular rotation speed versus time

10.11 Block diagram of a vector controller for an induction motor 
10.12 Induction motor speed, duty cycle, theta and the three phase currents versus time

$\begin{array}{lll}10.13 & \text { Induction motor speed variation with time }\end{array}$

$\begin{array}{lll}10.14 & \text { The system used for the overvoltage study } & 217\end{array}$

10.15 Line voltage profile for double pulse for the $42 \mathrm{~V}$ motor with $30 \mathrm{ft} \quad 218$ 14 AWG, 4-conductor braided shield cable@20kHz switching frequency

10.16 Line voltage profile for single pulse for the $42 \mathrm{~V}$ motor with $15 \mathrm{ft} 14$ 218 AWG, 4-conductor braided shield cable@20kHz switching frequency 
m.v.p. Magnetic Vector Potential

p.d.e. Partial Differential Equation

FE Finite Element

FEA Finite Element Analysis

IPS Integrated Power System

PEBB Power Electronic Building Blocks

PWM Pulse Width Modulation

WPT Wavelet Packet Transform

WT Wavelet Transform

DWT Discrete Wavelet Transform

WTCs Wavelet transform coefficients

Dq Direct Quadrature

THD Total Harmonic Distortion

DC Direct Current

AC Alternating Current

HF High Frequency

PM Permanent Magnet

SM Synchronous Motor

EMTP Electromagnetic Transient Program

e.m.f. Electro Motive Force

PI Proportional Integrator

mmf Magneto Motive Force 


\section{Chapter 1: Introduction}

\subsection{Introduction}

Naval ship power system is an autonomous power system. An autonomous power system is an energy system in which only local generation provides power to loads in the system. For autonomous power system, the energy generation capacity is limited. At the same time, the shipboard power system is a military system, where performance is the motivation for design of most loads, sources, distribution cables, control, and protection.

The US Navy has shifted priority from designing systems that offer increased capability to systems which are more affordable but enable improved performance, high system reliability and highly reconfigurable system. Integrated power system (IPS) concept is the latest topology under design and development for naval shipboard energy systems. The IPS combines the all electric ship initiative with direct current distribution, and power electronics building blocks technology.

The all electric ship initiative is becoming a principal design concept for future naval ships. It replace the ship hydraulic, and mechanical means by their equivalent electrical means to power virtually all ships subsystems including weapon system, control system, and utility functions. This concept offers advantages of increasing the ship war fighting effectiveness, reduce the life cycle cost (LCC), increase the energy system reliability, reduce the weight of the ship and reduce vulnerability to battle damage. Thus the integrated power system integrates all the shipboard generators and the system loads by means of a power distribution bus.

In the IPS, DC distribution system is used to transfer the power to different shipboard zones and within each zones there are different inverters and converters which are used to 
supply different AC loads and DC loads within this zone with their rated power. Power electronics based devices are used to convert generator AC output to DC distribution.

One of the proposed technologies for the IPS currently under development is the electronic building blocks (PEBB) technology. It is a technology that utilized to perform power energy conversion, inversion, and all other types of power modulation. PEBB is developed to replace the currently used conventional custom design of power electronic based converters, inverters, and controllers with modularized standard designs for the power electronic devices. This minimizes the outage time for the units and reduces the system maintenance costs.

Unlike the current shipboard power system design which is based on minimal interactions between the system components, the new suggested IPS consists of highly coupled subsystems closely interacting with each other. This approach provides potential advantages, which include the ability to optimize the whole system in real time which will allow the different subsystems to coordinate between each other and share the different subsystems resources such as regenerative energy with other subsystems. The benefits would be the reduction of the system failures, the overall operation and maintenance costs of the ship. It will also increase the shipboard power system reliability, security, and operational efficiency.

Naval Power systems have some inherent characteristics that make them different from typical utility power systems. Some of the characteristic of naval power systems includes [1-5]:

i. Fast controls is utilized to maintain the system frequency

ii. There is very little rotational inertia relative to the system load 
iii. Shipboard prime movers usually are faster than normal utility prime movers.

iv. Shipboard power system contains a large portion of dynamic, nonlinear, and electronic loads, relative to the power generation capability

v. Shipboard power systems are tightly coupled with mechanical systems, which require integrated modeling of both systems.

vi. Faults must be modeled consistently with the characteristics of naval power systems.

vii. Typical power system models are not usually appropriate for analyzing shipboard dynamics, which required more accurate calculations for shorter simulation time.

viii. The shipboard power system components parameters are highly frequency dependent.

These differences demand or necessitate the development of alternative techniques for modeling and analysis of shipboard systems. Figure 1 shows typical shipboard power distribution system architecture.

Navy Ships use delta connection configuration for both generation subsystem and distribution subsystem to ensure the continuity of the supply in the presence of a single phase to ground faults. The shipboard energy system is usually connected in ring configuration which increases the system reliability and allows dual path to supply power to any load.

The primary distribution system used on U.S. Navy ships is three-phase, 450 volts system, and 60 hertz (Hz) system. 
The secondary distribution system is a three-phase, $120 \mathrm{~V}, 60 \mathrm{~Hz}$ system. It is supplied from the $450 \mathrm{~V}$ system through transformer banks $[1,4]$.

Lighting distribution systems are usually three phase, $120 \mathrm{~V}, 60 \mathrm{~Hz}$ systems, and are supplied through transformer banks. Most ships also have $450 \mathrm{~V}$ and $120 \mathrm{~V}$, at $400-\mathrm{Hz}$ energy systems for supplying surveillance systems, weapons systems, aviation support equipments, and for servicing aircraft and landing craft. The 60 hertz and 400 hertz systems are connected together either by using a motor-generator sets or static solid-state frequency converters (SSFCs).

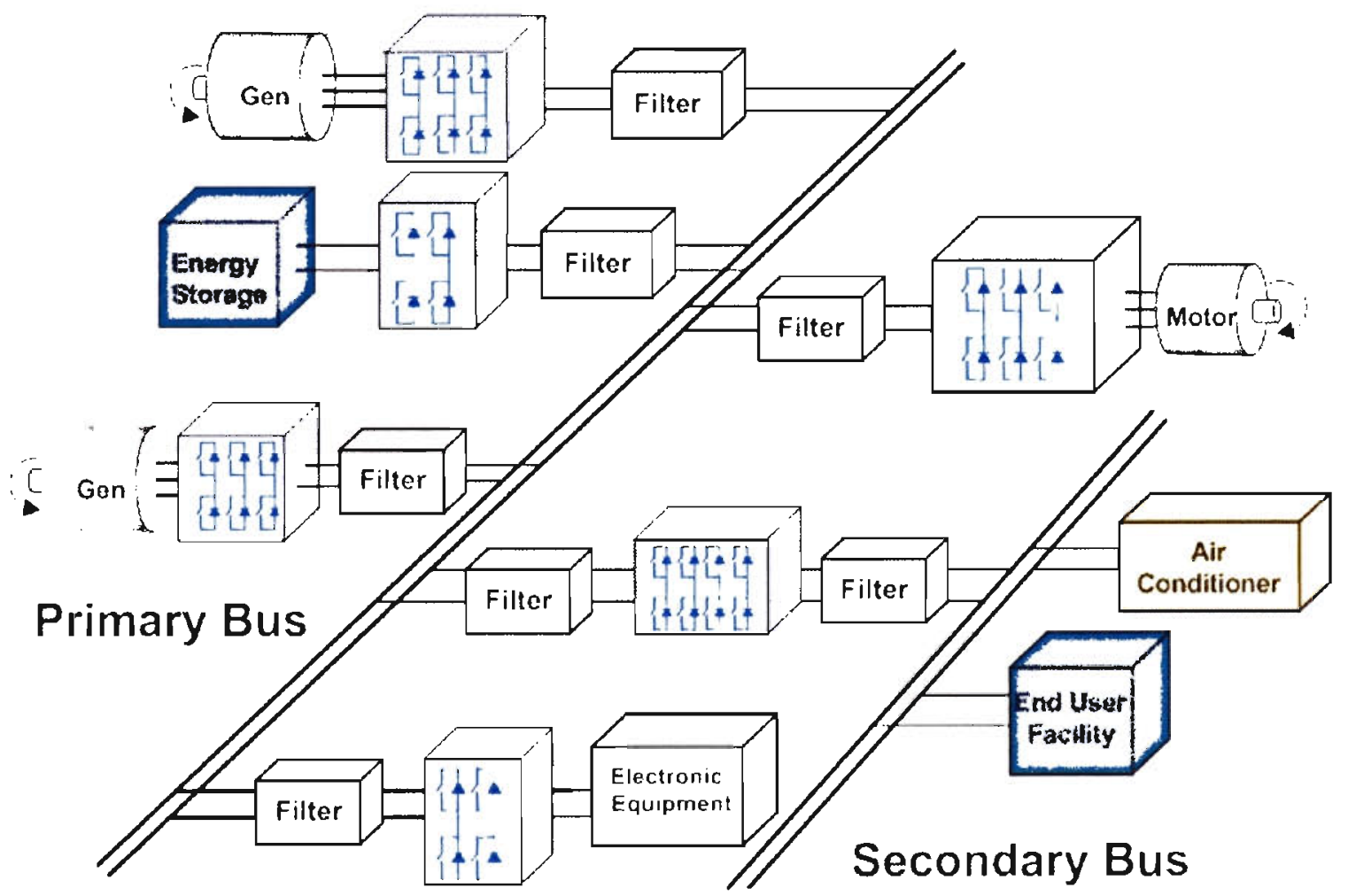

Figure 1.1 Naval power distribution system general architecture

Generators are connected to switchboards, which are composed of a number of switchgear units and components breakers. Switchboards provide a point of connection for ship service generators and local generator controls [4]. They are the first subsystem 
of the distribution system, and they supply power directly to large loads, vital loads, load centers, and power panels for further distribution to loads. Switchboards are usually located near the associated generators and are spread as far as possible to minimize the possibility of cascading failure. Ship generators are placed on different ship levels and are horizontally displaced along the ship to prevent total outage due to battle damage and to assure the continuity of the supply [5]. Tie-Bus circuits are used to connect the generator switchboards in a ring bus connection.

The other shipboard power system components are load center switchboards, power distribution panels, bus transfer units, transformers, and the cable network. Loads are connected directly to generator switchboards, load centers or power distribution panels. The load center switchboards provide energy to power panels or to individual system loads, either directly or via automatic bus transfers (ABTs) or manual bus transfers (MBTs). The bus transfers are used to provide vital loads with energy via dual paths. The $\mathrm{ABT}$ or MBT units are used to transfer a vital load supply from its normal supply path to an emergency alternate path in response to a contingency on the normal path.

Power distribution panels are located near their associated loads. They perform the control and protection tasks for selected portions of the power or lighting distribution systems and special power distribution systems.

Different load types exists in the shipboard power system with different importance this include induction motors, non-linear loads, and vital loads (e.g. weapons, radar, sonar, etc.).

Some aircraft carriers have $4160 \mathrm{~V}$, three-phase, $60 \mathrm{~Hz}$ generators and main switchboards that supply the $450 \mathrm{~V}$ AC system through power transformers. 
The shipboard protection system should have the ability to clear the fault as fast as possible by switching off only the loads affected by the fault and restore the service as quickly as possible by utilizing the suitable reconfiguration algorithm. The increase utilization of non linear loads and electronic loads will affect the protection system operations and reliability.

To conduct naval power system analysis, such as fault study, stability analysis, fault diagnosis, etc an effective simulation tool is needed [1-5]. Performance evaluation of integrated naval ship power system ship for a particular configuration is suggested to be evaluated through computer simulations. Therefore, it's essential to develop a computeraided modeling and analysis tools for a shipboard power system that take into consideration the shipboard system inherent characteristics. The integrated model is aimed to provide assessment tools for the navy power system under different operating conditions or uncertainties. The results are only as accurate as the mathematical models used. . It is therefore important to model each component of the system as accurately as possible.

Dedicated computer software are essential tools for making electric systems engineering studies, overvoltage studies, studies for the analysis of contingencies in electric systems, studies for the investigation of equipment failures and studies for the implementation of corrective measures in power systems. Currently there are different modeling techniques for shipboard power system components. Because of the NPS inherent characteristics that make them different from typical utility power systems, a more efficient modeling technique is required to avoid pure terminal models. These tools should have the ability to simulate the power system three main subsystems the apparatus 
subsystem, the control subsystem, and the protection subsystem in one simulation environment.

\subsection{Phase variable motor models}

Electric motors are the most commonly used as prime mover in many industries. In shipboard power system two main types of motors are usually utilized, induction motor and Permanent magnet synchronous.

Permanent magnet synchronous motor has a number of operational features which make them more desirable to be used for a variety of practical applications. This includes hybrid cars and shipboard power system applications. At the same time control strategies and fault tolerant motor drives studies are being developed to optimize the overall system performances under real-time dynamic conditions. For these studies, accurate and fast motor models are necessary.

The dq-model is a simplified modeling technique of rotating machines. It assumes that the flux linkage waveform of the three-phase winding is sinusoidal and that they have same amplitude and phase sequence. The effects of saturation, slotting, and the magnetic circuit geometry are ignored in such a dq-model. With the increase utilization of motor power electronic drives, researchers and engineers are paying attentions to additional details such as torque ripples, harmonics behavior of the motor, the incompatibilities of the motor with the load and high frequency effects. The dq model can't include all of this these effects. Harmonics generated in the system due to the non linear loads as well as power electronics drives will greatly reduce the accuracy of the dq model. Also, under fault conditions, when harmonics are presented in the voltage and 
current waveforms, the pure sinusoidal variation is removed and the dq-model becomes mathematically incorrect.

The FE model based modeling technique can take into consideration all geometrical, material and operational details of electrical machines [11]. When such a model is used in a coupled environment in a machine drive, it can be computationally intensive to evaluate the overall system performance at every time instant.

Mohammed et al. [11-19] suggest and developed an FE-based phase variable model for the motors to overcome the limitations of both $\mathrm{d}-\mathrm{q}$ and classical FE based model. This model combines the accuracy of the full FE model of the machine combine with fast computational time.

The physical FE-based phase variable model utilizes the rotor position dependence of inductance and back emf obtained from sequential FE solutions at rotor positions covering a complete ac cycle. By adding the cogging torque, the proposed FE-based phase variable model performed at the same accuracy level as the full FE model. The rotor position dependent parameters, inductance, flux linkage, and the cogging torque, are stored in tables and retrieved by table look up while performing drive system simulation. In such a way, the impact of space harmonics is included.

\subsection{Overview of simulation software for shipboard power system studies}

Dedicated computer software are essential tools for conducting energy systems engineering studies, overvoltage studies, studies for the analysis of contingencies in electric systems, studies for the investigation of equipment failures and studies for the implementation of corrective measures in power systems. The shipboard power system consists of elements with different physical nature, linear and non linear, electrical and 
non-electrical, such as generators, power electronics devices, cables, motors, and electronic devices. This variety requires a modeling and simulation tool that allows diverse physical systems to be modeled in one uniform simulation environment. EMTP/ATP, PSPICE, VTB, and MATLAB Simulink are the most used simulation packages by power engineers for electrical power systems, power electronics, and analog/digital circuit's simulation purposes. Due to the big difference in characteristics between the shipboard power system and typical utility power systems, a certain modifications is needed. This simulation tools are briefly explained in this

\subsubsection{Electromagnetic Transients Program (EMTP/ATP)}

Electromagnetic Transients Program (EMTP) or ATP is a computer program for simulating electromagnetic, electromechanical, and control system transients on multiphase electric energy systems [20-21]. Firstly developed in the late 1960's by Dr. Hermann Dommel, EMTP was released for general use in the early 1970's by the Bonneville Power Administration (BPA).

EMTP independent version, ATP (Alternative Transients Program) is the most widely used version of EMTP in the world today. ATP provides the user with the ability to model almost any kind of configuration for the electric network. The solution numerical algorithm is based on the Bergeron's method for the distributed parameters elements and on the trapezoidal integration rule for the linear lumped elements. Optimal reordering techniques and Gaussian elimination are used to handle sparse system matrices. It not only allows the detailed component modeling, but also the modeling of various types of fault disturbances and the integration of control system modules to the simulated system $[20,21]$ 
ATP contains standard models for the representation of linear and non-linear elements, like resistors, inductors, capacitors, transmission lines, power transformers, surge arresters, switches, diodes, thyristors, control systems, synchronous machines, etc... Details about different models can be found in the ATP Rule Book [20].

The ATP is not easy to interface with other software packages and in some what limited.

ATP was utilized to perform several shipboard power system studies $[7,8,10]$. It was used to perform faults studies, reconfiguration studies and switching operation. The models used in these studies were ATP standard components models. The Authors outlined that for integrated test system, the simulations suffered from numerical oscillation and signal quality issues. The Author suggested some techniques to mitigate this oscillation [10]. The Authors did not include the control system simulations or power electronics components in their studies.

\subsubsection{PSPICE}

SPICE is computer software package designed to simulate analog/digital circuits [22]. It was originally developed for the simulation of integrated circuits, from which it derived its name: Simulation Program with Integrated Circuit Emphasis [22]. PSPICE contains a model library for linear, discrete, passive and active electrical components, etc. for electrical network modeling and analysis.

Circuits and systems are simulated by connecting components together in a PSPICE electrical model. To simulate a shipboard power system network, the appropriate PSPICE models of the different system components are created from schematics of the standard available electrical elements and these models are connected together to create the system 
network. Each component model parameters are defined according to the specifications data of the system. In PSPICE, the interactive GUI allows us to modify an item by just clicking this item in the schematics and making any modifications.

In PSPICE, the parameters of interest can be selected in the schematics for monitoring. Software called Probe can be used to display the simulation results.

The program interface with other software packages and developing more detailed accurate models for different power system components is not an easy task. PSPICE was used in different shipboard power system studies [5, 9]. The studies were mainly concerned with fault studies, reconfiguration, and protection system related studies. The Authors utilized simplified power system models and didn't model any power electronics components or the control systems.

\subsubsection{Virtual Test Bed (VTB)}

The Virtual Test Bed (VTB) is software for prototyping large-scale, multi-technical dynamic systems [23]. It allows the evaluation of new designs without using hardware implementation. The main application driving the development of the VTB is the advancing in shipboard power system platforms. These platforms will rely heavily on power electronics based devices, distributed generation, energy storage, advanced power sources including fuel cells and gas turbines, and mix distribution networks that combine both DC power buses and $\mathrm{AC}$ buses and the fact that the shipboard power system is highly reconfigure system. VTB was developed to fill this need by providing a simulation environment that can be used to perform different system studies and its ability to include user custom build models. The software is not easy to interface with other software 
package and can not work with commercially available packaged for real time simulations.

\subsubsection{MATLAB/Simulink}

MATLAB is generic high level programming language with built in support for performing numerical computation. It was originally developed for solving linear algebra problems using matrices. Its name is derived from MATrix LABoratory [24]. MATLAB is a high-level programming compiler with built-in math functions support that enables writing computationally intensive codes faster and easier than the traditional programming languages such as $\mathrm{C}, \mathrm{BASIC}$ and FORTRAN. The MATLAB can execute codes by two different methods either by typing the commands directly in MATLAB command window, or by writing a source code file known as MATLAB script.

MATLAB contains built-in functions for solving problems requiring arithmetic, trigonometric, exponential operation, data analysis, digital signal processing, optimization, and 2-D and 3-D graphics and animation. A collection of functions in a certain area are known as MATLAB Toolbox. MATLAB and its toolboxes is a high level programming language shell which can be used for dynamic systems modeling, simulation, and data analysis in both commands based and graphical based forms [10]. There are a variety of commercially available MATLAB toolboxes for different types of applications and studies this include control system design, curve fitting, communication, and optimization.

Simulink is a toolbox extension of the MATLAB. Simulink is a graphical programming language for dynamic multidomain simulation and dynamic systems model Based Design. It provides an interactive graphical user interface and a customizable set of 
building blocks libraries for building system models based on their mathematical description [40]. Like MATLAB Simulink have different toolboxes which contains a collection of blocks for certain application like SimPowerSystems, SimHydraulics, Stateflow etc.

Real-Time Workshop (RTW) is a MATLAB toolbox which can automatically generates, packages, and compiles source code from Simulink models to create real-time software applications on a variety of systems [26]. By providing a code generation environment for rapid prototyping and deployment, RTW is the foundation for production code generation capabilities. By using RTW MATLAB can be easy linked to other software packages and can be used in real time environment.

Based on its benefits and features, MATLAB was selected as the system simulator.

\subsection{Classification of shipboard power distribution system studies}

In order to evaluate the interactions between different shipboard subsystems, models with different level of complexity were developed. These models can be utilized to perform different system studies such as system analysis, system performance optimization, power quality studies, reconfiguration studies, and system control design.

The simulations can be divided into three main categories, low frequency, high frequency, and harmonics studies. Low frequency study included load switching, faults, and normal loading conditions. The result of this type of studies can be used in relay setting selection, equipment rating selection etc.

High-frequency modeling is essential in the design of equipments to study impulse voltage and switching surge distribution, winding integrity, and insulation diagnosis and most often high-fidelity models in a bandwidth up to $10 \mathrm{MHz}$ are required for condition 
monitoring purposes. The study of high-frequency part of the spectra is necessary to include the stray capacitances effect on the system response.

Harmonic studies are essential in to design passive or active filters and to investigate the severity of certain power quality events on the system equipments.

\subsection{Real time simulations with hardware in the loop concept}

In order to achieve our research goals, the different components developed models are connecting together to perform system studies and to evaluate different subsystems interactions. These system studies provide an efficient ways for simulation, evaluation, and optimization of the different interacting subsystems within the naval shipboard power system. As mention before, the shipboard power system studies required more detailed components models to be used. With more detailed models used in the studies the global system model would be very computationally intensive, slow, and time consuming. Also, simulation of power semiconductor devices physical models is a very time-consuming and requires a very strong simulation tools.

The multi-distributed real time simulations concept allows us to overcome this drawback and to achieve faster simulations. Distributed computing or simulation, which is not a new concept, refers to distribution of a given task on a number of interconnecting computing elements. The simulation task is distributed between the different computation nodes through a communication link (e.g. Ethernet) [28]. In this approach the system computing nodes are coordinating their resources to achieve a faster simulation.

Traditional simulations of power systems performance evaluation studies, take long time due to the excessive computations. Despite today's computers high computational capabilities, the simulation does not run at anywhere near real time speed, since the 
platform, whether PC or workstation, is not specially designed and optimized for the simulation of power system. Also, all the power system simulations software mentioned above do not support parallel processing.

Thus to perform system-level testing which is one of the main steps in the development of a complex product (e.g. motor controller) in a comprehensive and cost effective way requires real-time simulations. So by utilizing this approach, the global system model can be distributed over several processors through a communication link (TCP/IP, FireWire etc). Also, this technique allows the different distributed subsystems to be executed at different update rates; which free some of the real time system resources for running the subsystems that required faster update. The multi-distribution modeling can include different operating systems. Real time operating system (e.g. QNX or Linux) will reduce the simulation time requirement significantly.

The multi-distributed environment can be achieved by utilizing the $\mathrm{C}$-language capability of the MATLAB coupled with a network communication protocols to develop a Simulink distributed simulation environment. The system global model is converted into a number of small subsystems, and each subsystem is assigned to one of the computing nodes. The results are then sent to a main machine for display.

\subsection{Literature review}

This section provides a literature review of previous work on different topics with in this dissertation. Due to the fact that this dissertation deals with many topics and each topic has a sheer numbers of papers available, only the most relevant previous work is discussed. The references are sorted under the topic, which the dissertation author found 
most interesting in the context of the research and was used to select the research approach.

\subsubsection{Overview of the coupled field-circuit problems}

In the following literature review, the coupled field-circuit problems are studied from the viewpoint of power system and power electronics. Formulations for coupling between Field and Circuit were developed for modeling of voltage-supplied electrical machines in the early 1980 's. Power electronics were represented in the FE- Circuit coupling during the late 1980's and early 1990's for simple implementation [29].

Tsukerman et al. discussed the formulations, terminology and numerical methods in the coupled field-circuit problems [30]. The magnetic vector potential formulations with filamentary and solid conductors were outlined. The filamentary or stranded conductors consist of several turns of thin wire connected in parallel. In order to simplify the analysis, the eddy currents effects in filamentary conductors can be ignored, but the current density is assumed constant. In the solid conductors eddy currents cannot be ignored and it have to be included in the analysis.

Meunier presented a generalized formulation for coupling two-dimensional finite element analysis with solid or filamentary conductors using sinusoidal voltage or current sources [31]. The method was later developed further for time-stepping analysis allowing resistive and inductive components in the external circuit [32]. The unknown variables of the formulation were the magnetic vector potential, current in the filamentary conductors and inductors, and voltage drop over the solid conductors.

In electrical machine FE model the magnetic field is excited by currents in the coils. However, it is often more appropriate to consider the source feeding circuit in the 
analysis which required the coupling between the two systems of equations. At first, magnetodynamic formulations using complex variables were presented for ideal sinusoidal supply; later on, approaches for transient simulation were derived in order to model nonsinusoidal voltage waveforms. The phase windings in the stator and rotor are generally modeled as filamentary conductors, and the rotor bars in cage induction machines or damper windings in synchronous machines are modeled as solid conductors with eddy currents [32].

Vassent et al. included the nonlinearity of the iron and the impedances of the end-ring in the FE analysis of squirrel cage induction machine using either the time-harmonic [33] or time-stepping approach [34].

Power electronics can be considered in the coupled field-circuit analysis in several ways. The simplest approach is to define the supply voltage waveform with respect to time or position and use it in the simulation. However, modeling the interaction between the electrical machine and the drive board requires modeling the nonlinear semiconductor devices. Usually, the switching devices are represented in the circuit domain as binaryvalued resistors, the value of which depends on the state of the switch. A distinction is often made between diodes and externally controlled switches because of the differences in operation principle. In the simulation of circuits with diodes or rectifiers, the time step must be adapted to the switching instants in order to prevent negative overshoots in the current [35]. For the externally controlled switches, synchronization of the time steps is simple, since the switching instants are already known in advance.

Piriou and Razek modeled the diode operation by means of an exponential function and applied it to the simulation of a simple circuit consisting of a magnetic coil, voltage 
source and diode [35]. Later on, the method was extended for rotating machines and three-dimensional geometries [36]. The controllable switches were modeled in the same manner, but the switching instants had to be defined before the simulation.

Coupled field-circuit analysis was also applied in modeling power system components operation with external and internal faults. Some approaches utilize a time control switch to initiate the internal or external fault. In the simulation of circuits with switches, the time step must be adapted to the switching instants in order to prevent negative overshoots in the current $[37,38]$.

\subsubsection{Transformer modeling}

Modeling of power transformer represents a challenge to engineers. There are different models for transformers with different modeling details for different studies. These models were designed to include the effects of core saturation, ferroresonance, hysteresis, and insulation issues. Transformer modeling can be classified into two main categories normal transformer modeling and transformer with internal winding fault modeling.

The main streams in the modeling for analysis and design of power transformers are classified as follows [39]:

The first approach represents the transformer using its DC resistance, leakage inductance of the windings, and an ideal transformer [40, 41]. A Linear or nonlinear impedance is used to represent the characteristics of the core. The method uses formulas to calculate the self and mutual inductances between the windings based on the manufacturer data. But the iron core effect, however, makes the values of inductances 
very close to each other which results in ill-conditioned equations. This problem has been efficiently solved by subtracting the common flux when computing the inductances.

Transformer modeling based on self and mutual inductances was developed based on the mutual coupling concept $[42,43]$. This model represents adequately the leakage inductances of a transformer but sustain difficulties when representing the iron core related effects.

Transformer modeling based on the principle of duality represents accurately the iron core effect, but can not model the leakage inductances properly [44, 45].Topologicallycorrect equivalent circuit models can be derived from magnetic circuit models using the principle of duality, with the duality transformation being directly performed as a topological exercise.

Transformer modeling based on measurements models the transformer as a terminal equivalent in a wide band spectrum of frequencies [46-48]. Methods of this group match the parameters of the assumed model structure with the transformer experimental frequency response. Tests are made for determination of the model parameters in the frequency or time domain. Weak basis for generalization is the major drawback of those methods [39].

Finite Element (FE) based modeling use two or three-dimensional finite element method for analysis of electromagnetic fields inside the power transformer [49, 50]. FE based modeling is very accurate but rather geometry oriented with very intense and slow simulations.

Some transformer modeling technique combines between two or more modeling approach [39]. The developed model combined the leakage inductances approach with 
the principle of duality approach. The model parameters were calculated on turn-to-turn basis. The capacitance effect was taken into consideration.

The presence of harmonics in the energy system can be a source of a variety of undesirable effects. Transformers are most affected with this problem and may need to be derated to as much as $50 \%$ capacity when feeding loads with extremely distorted current waveforms and may reduce their service life. The IEEE/ANSI C57.110-1998 standards states that a transformer subject to nonsinusoidal load current having more than $5 \%$ total harmonics distortion needs to be derated [52]. Transformer parameters are usually frequency dependent and modeling transformers under distorted supply should take into consideration this frequency dependence.

Gustavsen developed a modeling technique to take into consideration the frequency dependence of the transformer parameters [53-57]. The transformer admittance matrix is measured in the frequency domain using a network analyzer and a dedicated measurement setup. Then admittance matrix was then approximated with rational functions using Vector Fitting and the result is used to develop an EMTP-type compatible model suitable for transient studies.

The second portion of transformer modeling review deals with the modeling of transformer with internal faults. When a fault occurs inside the transformer, the magnetic flux distribution is fundamentally altered and this will affect the transformer parameters. Thus, most existing transformer models cannot adequately represent a transformer with an internal winding fault. However, some researchers have made some efforts to modify or take into consideration the fault related effects on the transformer. 
Two approaches are used in modeling transformers with internal faults, linear based approach and FE based approach.

Bastard et al. presented a model based on the self and mutual inductances [58]. The principle used to model a fault between a coil turn and earth or between turn and turn is to divide the faulty winding. For transformer turn to ground fault the winding is divide it into subcoils, for turn-to-turn internal fault the faulty winding is divided into three coils. The model transformer was modified to represent the added windings. The author assumed knowledge of how to calculate the leakage factors between the various coils of the transformer. This model is EMTP compatible, and is easy to implement. However it's linear model and assumes that no saturation occurs in the core during the faults and linear change in the flux distribution.

Wang and Butler presented a coupled field-circuit model for single phase transformer with internal faults [38]. The terminal behavior of the transformer was obtained by indirect coupling of the field-circuit simulations.

\subsubsection{Application of wavelets in power system}

With the increase utilization of highly nonlinear electronic controlled devices in power systems, the amount of waveform distortion has been found to be more significant. Harmonics generated by these transients can have a wide frequency bandwidth, from high-frequency transients and edges to slowly varying harmonic components. Hence, analysis only in the frequency or time domain alone is not sufficient to capture features that are spread within a wide bandwidth.

The wavelet transform which is a time-frequency digital signal processing technique provides a local representation in both time and frequency domain of the nonstationary 
signals. Therefore, it is suitable for analyzing a signal where time and frequency resolution is needed, unlike FFT which gives a global presentation of the signal.

Wavelet was implemented to two main areas in power system namely power system relaying and power system quality studies.

Santoso and Hofmann tested and demonstrated the use of wavelet transforms for detecting various power lines disturbances [59]. The basis of disturbance classification scheme, using the squared wavelet transform coefficients, was also described.

Robertson et al. introduced the use of the wavelet transform for the classification and analysis of electromagnetic power system transients [60]. He pointed out that Wavelet features facilitate the detection of physically relevant feature in the transient signals to characterize the source of the transient or the state of the post disturbance system.

Yoon and Devaney demonstrated how to obtain the power and the rms values directly from the wavelet transform analysis results for each element voltage and current pair [63]. The original signal frequency decomposition into various wavelet decomposition levels was achieved using IIR filters. This technique was later extended to measurement of reactive power and distortion powers [64].

Many authors demonstrated the utilization of discrete wavelet transform (DWT) to classify different transients [65-69]. The key advantages of the DWT are its ability to provide a local representation of the nonstationary fault signals for normal and faulty operating conditions by using the variable windowing technique.

Issues regarding frequency characteristics of wavelets were outlined [70]. The authors pointed out that the selection of sampling frequency and the number of data points is important in the wavelet based study. energy distributions in wavelet decomposition 
levels were studied and it was found that the total energy was the same but distributed between the different wavelet energy levels.

Zhu et al. calculated and used the energy distribution of the wavelet at each decomposition level to extract power-quality disturbance features [72]. Based on these features, rule bases are generated for extended fuzzy reasoning.

Littler, and Morrow demonstrated the analysis properties of the discrete wavelet transform (DWT) and wavelet packet transform (WPT) [73]. The authors outlined that both transforms offer attractive properties for the compression of power system disturbances especially WPT which provides exciting prospects for adaptable feature extraction algorithms.

Gaing proposed a prototype of wavelet-based neural-network classifiers for power disturbance characterization and classification using the Parseval's Theorem to calculate the different wavelet frequency bands energy [74].

Dash and Panigrahi proposed a new approach for detection, localization, and classification of power quality disturbances in a power distribution system [75]. This approach utilized generalized S-transform with a variable window as a function of PQ signal frequency to generate contours and feature vectors for pattern classifications.

Hamid et al. proposed a wavelet packet transform (WPT) based approach to calculate the root mean square (rms) value of distorted waveforms, total harmonic distortion, and for power measurements [77]. The algorithm can simultaneously calculate the individual frequency bands rms values from the wavelet coefficients associated with each element voltage current pair. The advantage of the WPT is that it can decompose a distorted 
waveform into equal width frequency bands, which is important for identification of different harmonic components and their influence on the system.

\subsubsection{Integrated motor drive modeling}

Research on high level modeling, new converter-inverter topologies, and control strategies are the major research areas in electrical drives. A system consisting of a loaded motor driven by a power electronics converter through a cable is a complex and nonlinear system.

Toliyat et al. suggested a method to estimate the voltage distribution among the windings of an inverter fed random wound induction motor supplied through a long cable [78-80]. The authors developed a high frequency models for the inverter, cable, and the motor, and the transient study was performed using ATP as a system simulator. The induction motor high frequency parameters were calculated using $\mathrm{FE}$ analysis and the parameters of the cable were computed using ATP cable constants routine.

Suresh et al. suggested a method of calculating the voltage distribution among the windings of random wound induction motor supplied through a pulsewidth modulating (PWM) voltage source through feeder cable [81]. The motor high frequency model parameters were obtained from FE analysis. The transient study was performed using SABER simulation package to study the rise time of the PWM wavefront on the voltage e distribution among the motor windings.

Grandi et al. analyzed inverter-fed ac motor drive to evaluate the conducted electromagnetic interferences at both the dc and ac sides of the inverter [82]. High frequency lumped model for the inverter and the motor windings was used to conduct the study. The inverter model includes parasitic elements of power switches, passive 
components and connecting wires. The circuit model of the motor windings takes into account the turn-to-turn and turn-to-ground stray capacitances. The motor model fitting parameters was obtained using phase-to-phase and phase-to-ground impedance measurements. The overall system model response was then evaluated for a $10 \mathrm{kHz}$ sixstep modulated inverter - fed ac motor drive.

Moreira et al. studied the overvoltage problem in pulsewidth modulation drives with long cables [83]. The frequency responses of the cable and the motor input impedances were obtained experimentally and suitable models are developed to match the experimental results. The cable was model using several lumped lossy segments connected in series.

\subsubsection{Modeling of induction motor with internal faults}

Induction motors are widely used in many industries because they are cost effective and mechanically robust. Because of costly machinery repair, down time, and safety consideration, early detection of induction motor internal faults is highly desirable.

In general, fault diagnosis of induction motors has concentrated on sensing failures in one of three major components, the stator, the rotor, and the bearings. Approximately $36 \%$ of induction motor failures are caused by failure of the stator windings, and these faults usually begin as undetected turn-to-turn faults in a coil, which progress to phase-tophase or phase-to-ground short circuit faults. Rotor failures account for $5-10 \%$ of total induction motor failures [83-85].

Modeling of induction motors with internal faults is the first step in the design process of the fault detection systems. With the presence of internal faults the modeling of induction motor is very complicated because the magnetic field distribution totally 
changes inside the machine. There is several modeling approach for induction motor with internal faults. They can be divided into two main approaches, dq based techniques, and FE based techniques.

Jang and Park utilized coupled FE model to study the characteristic frequency of the rotor vibration generated by broken rotor bar, static, and dynamic rotor eccentricity fault conditions [87]. The Author conclude that each fault source can be distinctively identified by observing the variation of the corresponding characteristic frequency of the rotor vibration, for signal as will as several fault sources together.

Watson et al. described how Finite Element packages may be used to simulate rotor faults namely broken bar faults [88]. The authors utilized a simulation method that minimizes the effects of speed ripple and rotor skew on the simulation accuracy.

Bangura and Demerdash built a Time-Stepping Coupled FE-State Space (TSCFE-SS) induction motor model for predictive non-invasive diagnosis and characterization of effects of rotor broken bars and end-ring connector segments which rigorously includes the full impact of magnetic saturation $[89,90]$. The model has been used to predict the characteristic frequency components which can he used to diagnose rotor bar and connector breakages.

An induction motor with internal fault models were developed as an alternative to FE based methods [91, 92]. The models are based on coupled magnetic approach by considering that the current in each bar is an impendent variable. The models neglect the saturation effect, eddy-currents, windage and friction losses, and assume uniform (magnetically smooth) air gap, uniformly distributed cage bars, and insulated rotor bars. 
These assumptions affect the accuracy of the results especially in the case of nonsinusoidal voltage source.

Manolas and Tegopoulos developed a method for modeling the performance of a squirrel cage induction motor under any asymmetrical operating condition [93]. The system of nonlinear voltage and torque differential equations representing the machine mathematical model was analyzed directly without transformation by using a digital numerical solution to compute the steady-state and the transient state. The model was used for broken bars and rings studies.

R. Fiser and S. Ferkolj presented a magnetodynamic FE based model for predicting the performance of induction motor under electric and magnetic asymmetry due to broken rotor bars [94]. The FE model was used to calculate the magnetic vector potential, flux density; force components, rotor and stator currents, and mutual and leakage inductance. The author takes into consideration the magnetic core materials nonlinearity. The model assume sinusoidal voltage source and does not take into consideration the instantaneous changes in the system voltage and currents during the fault.

\subsection{Problem statement}

Electrical machine designers and industry researchers face an increasing demand of considering many physical effects and operational parameters more accurately. The original motivation for this work was to simplify the procedure of modeling, simulating, and optimizing the design electrical shipboard power system components.

The main problem addressed in this dissertation was the development of a methodology for studying and evaluating the integrated shipboard power system behavior in real time environment. 
This research addressed this problem by using coupled-FE to model and assesses the different electromagnetic components behavior under normal and faulty operating conditions. This procedure utilized the limited electromagnetic device available information such as factory test reports, core type and core dimension to develop FE based models of the different shipboard power components. Then the developed FE models were then used to obtain the operational parameters. The operational parameters were then used to build a phase variable models for the different components. The phase variable models considered many magnetic core related effects that usually ignored by other modeling techniques. The developed phase variable models were then coupled together to perform system studies using the system simulator. This global model can be used in a distributed real time environment.

The developed models can be classified according to their target applications as low or high frequency models. These models were then utilized to perform system studies, to evaluate the overall system performance, and to develop a methodology's system indices. This includes power quality indices, overvoltages indices, and components behavior under faults.

With the increased utilization of power electronic devices and non-linear loads in the shipboard power system, the harmonic distortion in the system increased as well. In order to evaluate the system harmonic response and to classify the harmonics related events and faults conditions an efficient digital signal processing technique is needed. Analysis of naval shipboard power system waveforms only in the frequency or time domain alone is not sufficient to capture these waveform features that are spread within a wide bandwidth. Wavelet transform provides a local representation of nonstationary 
signals. Therefore, it is suitable for analyzing a signal where time and frequency resolution is needed. A different wavelet based classification algorithms were developed for power quality and faults studies. This includes feature extraction as well as evaluation of the distorted waveform indices.

A real time distributed simulation shell was then developed to conduct shipboard power system studies. This studies can be pure software based or can include within the simulation loop the actual hardware. The software subsystem is interacting in real time with the hardware subsystem. This was achieved because of the small time step achieved by using the distributed simulation. Implementation examples of using these developed tools for energy efficient and robust shipboard power system operation and control were outlined and discussed.

The methodology is presented in this dissertation for utilizing the coupled Fieldcircuit computation of electromagnetic devices to evaluate and predicate the shipboard power system components future behavior and to optimize its performance to meet its required functionality. And to use this FE models to develop an accurate detailed phase variable models that can be used in chosen system simulator. The system studies are performed under real time simulation shell. This shell will enable us to combine actual hardware in the simulations and to evaluate the interaction between the software and hardware subsystems.

\subsection{Originality and significance}

The scientific contribution of this study comprises the following:

1. Methodology for coupled simulation of magnetic field, circuits and control systems to model accurately shipboard power system components. 
2. The development and evaluation of FE based models for shipboard power system components for abnormal and internal fault studies.

3. The development and evaluation of detail models for shipboard power system components for low and high frequency studies using parameters obtained from FEM. These models can be used in the chosen system simulator.

4. The development and evaluation of a real time distributed simulation environment to perform integrated system studies. The real time execution will allow the integration of the actual hardware in the simulation loop. Using this real time shell we represent the interaction between the hardware subsystem and the control system.

5. Validation of the applicability of the methodology by performing different type of system studies relating to shipboard power system.

6. The development and evaluation of a methodology for fault feature extraction and harmonic behavior evaluation for different shipboard components using wavelet based algorithms.

\subsection{Organization of the dissertation}

This dissertation has two main parts: background information and contributions. Chapters

$1,2,7$, and 9 contain background material, state of the art knowledge and all necessary information used to make decisions in this research. Chapters $3,4,5,6,8,10$ contain the contributions and results of this work.

In addition to the outline of the work in this chapter, the other chapters include:

Chapter 2 presents background material for coupled field-circuit modeling and operational parameters evaluation. 
Chapter 3 deals with modeling of different components by using FEM and how to use FEM to calculates the elementary quantities used in modeling

Chapter 4 deals with modeling of different components with internal faults by using coupled FEM methods

Chapter 5 describes system components models development for low frequency application.

Chapter 6 describes high frequency modeling and theory covering frequency-dependent parameters characteristics, vector fitting and how the characteristics are included in components modeling.

Chapter 7 describes theory regarding wavelet, discrete wavelet transform, and wavelet packet transform together with the wavelet representation of the waveform root indices including the mean square value (rms) and total harmonic distortion (THD) values.

Chapter 8 deals with the application of wavelet transform techniques in power system transients and fault classification, and power quality studies.

Chapter 9 describes the real time with hardware in the loop simulation system

Chapter 10 presents some practical system studies using the real time distributed simulation and discusses the results and optional solutions.

Chapter 11 suggests topics for further research, and

Chapter 12 contains the main conclusions from this work. 


\section{Chapter 2: Coupled Field-Circuit Modeling}

\subsection{Introduction}

This chapter describes the Finite element (FE) based computational methods that are used to simulate the terminal behavior of the electromagnetic equipments. FE is numerical solution technique for partial differential equations. FE is used in electromagnetic field analysis to solve the electromagnetic field partial differential equations. The FE analysis has been coupled to circuit simulation. This technique allows the simulation of the device operating conditions with the real power supply connections. In this technique the device finite element model is coupled with external circuit equations and solved the resultant equations simultaneously to obtain the device behavior. The field-circuit equations are coupled together either by direct coupling or indirect coupling $[30,36]$. In direct coupling, the electric equations are directly coupled with the field calculation and solve simultaneously using the same program. In indirect coupling, the field analysis is performed by a stand alone program, while the electric simulation and coupling are handled in another separate program which utilizes the parameters calculated from the FE solutions.

\subsection{Finite element formulation}

In the model of the electromagnetic devices, the magnetic field in the iron core, windings and air gap is solved by the two-dimensional finite element method and coupled with the windings voltage equations. The resulting equations are then solved by a timestepping approach, while the Newton-Raphson iteration is utilized for handling the nonlinearities. 
The magnetic field inside the electromagnetic device is governed by the Maxwell's nonlinear partial differential equation $[29,30]$ :

$$
\begin{aligned}
& \nabla \times E=-\frac{\partial B}{\partial t} \\
& \nabla \times H=J_{e}+J_{c}+\frac{\partial D}{\partial t} \\
& \nabla \cdot D=\rho \\
& \nabla \cdot B=0
\end{aligned}
$$

Where,

$E$ is the Electric field strenght $(\mathrm{V} / \mathrm{m})$

$B$ is the Magnetic flux density $\left(\mathrm{Wb} / \mathrm{m}^{2}\right)$

$H$ is the Magnetic field intensity $(A / m)$

$J_{e}$ is the conduction current density $\left(A / \mathrm{m}^{2}\right)$

$\frac{\partial D}{\partial t}$ is the displacement current density $\left(A / \mathrm{m}^{2}\right)$

$J_{c}$ is the convection current density $\left(A / \mathrm{m}^{2}\right)$

$D$ is the Electric flux density $\left(\mathrm{C} / \mathrm{m}^{2}\right)$

$\rho$ is the Electric Charge density $\mathrm{C} / \mathrm{m}^{3}$

The magnetic field intensity $(\mathrm{H})$ is related to the magnetic flux density (B) by the following equation:

$$
H=v B \quad(\mathrm{~A} / \mathrm{m})
$$

$v$ is the magnetic reluctivity, and it is a material dependent. 
The magnetic flux density can be expressed as the curl of the magnetic vector potential (A).

$$
B=\nabla \times A
$$

If we substitute (2-6) into (2-1), we get

$$
\nabla \times E=-\frac{\partial}{\partial t}(\nabla \times A)
$$

Rearrange (2-7) we get

$$
\nabla \times\left(E+\frac{\partial A}{\partial t}\right)=0
$$

Since the sum of the two vector quantities in the parentheses of (2-7) is curl free, it can be expressed as the gradient of a scalar electric potential (V).

$$
E+\frac{\partial A}{\partial t}=-\nabla V
$$

From which we obtain

$$
E=-\nabla V-\frac{\partial A}{\partial t} \quad(\mathrm{~V} / \mathrm{m})
$$

If we substitute (2-5) and (2-8) into (2-2) and make use of the constitutive relations $H=v B$ and $D=\varepsilon E$. We have the fundamental equation of the vector potential formulation for electromagnetic field

$$
\nabla \times((v) \nabla \times A)=J+\varepsilon \frac{\partial}{\partial t}\left(-\nabla V-\frac{\partial A}{\partial t}\right)
$$

Equation (2-9) is solved in the domain studied with the boundary conditions and the constitutive relationships.

The current density is determined from the material equation

$$
J=\sigma E
$$


Where $\sigma$ is the electric conductivity. Combining (2-2) with (2-6) gives

$$
\nabla \times E=-\frac{\partial}{\partial t} \nabla \times A
$$

From which we can define the current density as

$$
J=-\sigma\left(\frac{\partial A}{\partial t}+\nabla V\right)
$$

Taking the divergence of both side of (2-12), and then use the fact that the divergence of the curl of any vector field is zero, we get

$$
\nabla \cdot\left(\sigma\left(\frac{\partial A}{\partial t}+\nabla V\right)=0\right.
$$

Equations (2-9) and (2-13) represents the fundamental equation of the vector potential formulation for electromagnetic field.

The two equations are then solved using Finite element method (FEM). The finite element analysis of any problem involves basically four steps [29]:

- discretizing the solution region into a finite number of subregions or elements,

- deriving governing equations for a typical element,

- assembling of all elements in the solution region, and

- solving the system of equations obtained

The two-dimensional geometry is covered by a finite element mesh, consisting of first or second-order triangular elements. If possible, the cross section of the electromagnetic device is divided in to n symmetry sectors, from which only one is modeled using FEM and symmetry constraints are set on the periodic boundary.

In the finite element method, the approximation for the magnetic vector potential and scalar potential are given by 


$$
\begin{gathered}
A=\sum_{j=1}^{N_{n}} \lambda_{j} a_{j} \\
V=\sum_{i=1}^{N_{m}} \alpha_{i} v_{i}
\end{gathered}
$$

where $N_{n}, N_{m}$ are the total number of free nodes in the finite element mesh, $\lambda_{j}$ and $\alpha_{\mathrm{j}}$ are the classical approximation functions of edges and nodal elements; $a_{j}$ and $v_{j}$ are the magnetic vector potential and scalar potential in node $\mathrm{j}$.

The shape functions have value 1 at node $\mathrm{j}$ and 0 at all the other nodes. Between the nodes in the surrounding elements, the shape function is fitted by first- or second-order polynomials, depending on the order of the elements. Outside the associated elements, the value of a single shape function is zero.

Using Galerkin's method, (2-9) and (2-13) can be written in the following matrix form:

$$
\left[\begin{array}{cc}
S & C \\
0 & T
\end{array}\right]\left[\begin{array}{l}
a \\
v
\end{array}\right]+\left[\begin{array}{cc}
G & 0 \\
C^{T} & 0
\end{array}\right] \frac{d}{d t}\left[\begin{array}{l}
a \\
v
\end{array}\right]=\left[\begin{array}{c}
F \\
P
\end{array}\right]
$$

The solution of the matrix equation (2-16) gives the vector field which satisfies (2-9) and $(2-13)$.

\subsection{Electric circuit equations}

The FE analysis has been coupled to circuit simulation. This external circuit coupling allows us to simulate the operating conditions of the electromagnetic device with real power supply connections.

In general any electric circuit can be represented by the following integrodifferential matrix equation: 


$$
\left[E_{m}\right]=\left[r_{m}\right]\left[I_{m}\right]+\left[L_{m}\right] \frac{\mathrm{d}}{\mathrm{dt}}\left[I_{m}\right]+\left[\frac{1}{C_{m}}\right] \int\left[I_{m}\right] d t+\left[\gamma_{m}\right]+\frac{d}{d t}\left[\psi_{m}\right]
$$

Where, $E_{m}$ is the vector of voltage sources in each electric mesh m.

$r_{m}$ is the matrix of resistance (symmetric)

$L_{m}$ is the matrix of inductances (symmetric)

$I_{m}$ is the vector of electric current in each mesh.

$\gamma_{m}$ is the non-linear voltage drop in the electrical circuit

$C_{m}$ is the matrix of capacitances

$\psi_{m}$ The flux linkage in the coil

In matrix $r_{m}$, elements $r_{i j}(K \times K)$ are calculated by,

$\mathrm{r}_{\mathrm{ii}}$ is obtained by addition of resistances in the mesh $\mathrm{i}$

$r_{i j}$ is obtained by addition of resistances which belong to the meshes $i$ and $j$ with the sign

+ if the currents of meshes $i$ and $j$ are in the same direction, with the sign - otherwise.

$L_{i j}(K \times K)$ is formed the same way as the resistance matrix.

$C_{i j}(K \times K)$ is formed the same way as the resistance matrix.

$K$ is the number of electric meshes

Now equations are set, we can put them together to obtain the coupling system.

Equation (2-17) can be written in the following form:

$$
\left[E_{m}\right]=\left[X_{0}\right] \int[i] d t+\left[X_{1}\right][i]+\left[X_{2}\right] \frac{\mathrm{d}}{\mathrm{dt}}[i]+\frac{d}{d t}[\psi]+[\gamma]
$$




\subsection{Relation between magnetic and circuit equations}

For a winding with thin conductor, the current density can be expressed by (2-12). To represent the current density, we define the vector of turn's density $\mathrm{N}$ (with $J_{0}=N i$ ). Its module is given by the ratio of number of turns to the winding section and its direction by the spacial orientation of the winding. The relation between the winding voltage $V_{0}$ and the current is obtained by the projection of vector $\mathrm{N}$ on $J_{0}$ and by an integration on the whole winding volume $\Omega_{o}$. Under these conditions, the winding voltage is given by:

$$
V_{o}=r_{o} i+\frac{d}{d t} \int_{\Omega_{s}} A \cdot N d \Omega
$$

In this Equation the integral term represents the flux linkage $\psi, \psi$ is a non-linear function of the current due to the magnetic material non linear properties.

\subsection{Coupling the field and circuit equations}

In the general case the magnetic and electric equation are solved simultaneously. The electromagnetic field equations are solved using a formulation with magnetic vector potential. The coupling is obtained by the conductor current expressed in terms of current density and the flux linkage expressed from the vector potential. Two approaches can be considered for choosing the unknown of the coupled system. In the first approach the coupled equation system is only expressed in terms of the vector potential and scalar potential (V). In this approach the resultant equations are symmetric positive definite but the electric circuit non-linearity cannot be considered simultaneously with that of the magnetic circuit. In the second approach, the magnetic variables and the currents are kept as unknowns. In this approach the resultant coupled equations are is positive definite, but 
the non-linearities of the magnetic materials and electric circuit can be considered simultaneously. The second approach is utilized through out this thesis. Fig. 2.1 shows the coupling between the finite element, and the external circuit connections.

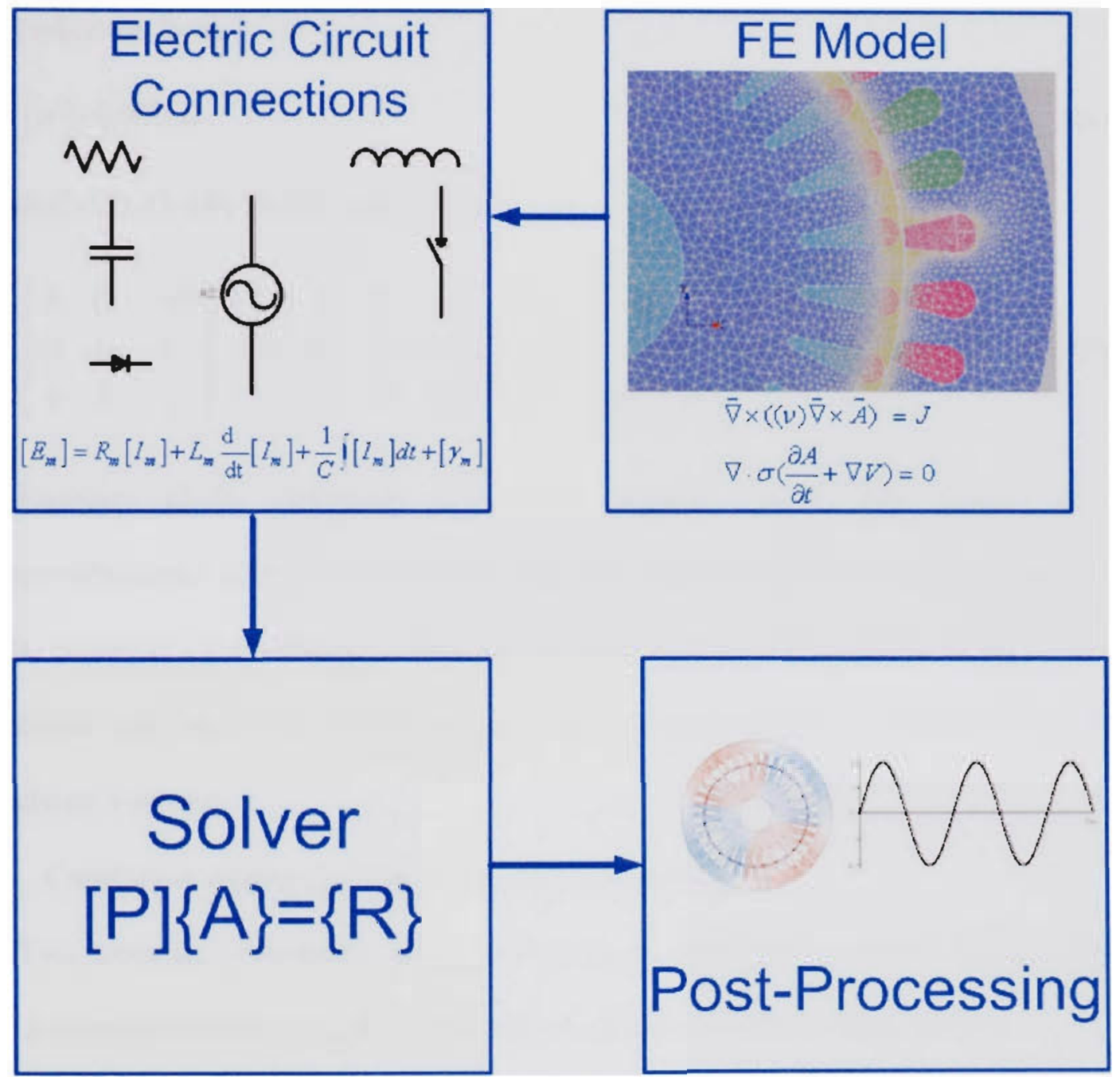

Fig.2.1 Schematic of the coupling between FEM and external circuit connections

The current density in the last term of (2-16) is expressed as a function of the current with help of vector $N, F$ as follow:

$$
[F]=[D][i]+\left[F^{\prime}\right]
$$


The element of matrix D depends on vector $\mathrm{N}$ and functions given by (2-14) and (215). The Flux $\psi$ can also be expressed from the integral of $(2-12)$ and the vector potential circulation a. For a system with several winding we can shows that the vector $[\psi]$ takes the form:

$$
[\psi]=[D]^{T}[a]
$$

From (2-12), (2-18), (2-20), and (2-21) we get:

$$
\left[\begin{array}{ccc}
S & Q & -D \\
0 & G & 0 \\
0 & 0 & r_{m}
\end{array}\right]\left[\begin{array}{l}
a \\
v \\
i
\end{array}\right]+\left[\begin{array}{ccc}
T & 0 & 0 \\
Q^{T} & 0 & 0 \\
D^{T} & 0 & L_{m}
\end{array}\right] \frac{d}{d t}\left[\begin{array}{c}
a \\
v \\
i
\end{array}\right]+\left[\begin{array}{c}
0 \\
0 \\
C_{m}^{-1}[i d t
\end{array}\right]+\left[\begin{array}{c}
0 \\
0 \\
\gamma_{m}
\end{array}\right]=\left[\begin{array}{c}
F^{\prime} \\
P \\
E_{m}
\end{array}\right]
$$

Equation (2-22) represents the whole coupled circuit-field system. In the integrodifferential (2-22) the unknowns are the circulation of the vector potential, the scalar potential for the magnetic domain, the mesh current for the electric circuit. All the equations will be solved simultaneously and the mathematical coupling between the equations is strong.

\subsection{Conductor representation in coupled-FE method}

Two cases are considered: solid conductors, in which eddy current can be induced, and a stranded conductor made of thin separate filaments without eddy currents.

\subsubsection{Solid conductors}

In the solid conductors, or massive conductors, eddy currents represent a significant part of the total excitation and they cannot be omitted from the analysis. Neglecting the source current density component from analysis, rearranging (2-9)

$$
\nabla \times(v \nabla \times A)+\sigma \frac{\partial A}{\partial t}+\sigma \nabla V=0
$$


In the two dimensional $\mathrm{FE}$ model, the magnetic vector potential (A) and the current density (J) are reduced to their $\mathrm{z}$ components. It follows that the value of the electric scalar potential is constant on the cross section of a two dimensional conductor and its value is a linear function of the $\mathrm{z}$ coordinate. If $L$ is the length of the solid conductor (along the $\mathrm{z}$ coordinate), $L \nabla V$ represents the potential difference $\Delta V$ induced between the ends of the conductor. Equation (2-13) can be integrated on each solid conductor region $\mathrm{k}$ where $\Delta V$ is constant. We obtain:

$$
\int_{S_{k}} J d s_{k}=I_{k}=\int_{S_{k}} \sigma \frac{\partial A}{\partial t} d s_{k}-\left(\nabla V_{k}\right) \int_{S_{k}} \sigma d s_{k}
$$

Where $I_{k}$ is the total current through solid conductor k. If we consider that:

$$
\begin{gathered}
\Delta V_{k}=L_{k} \nabla V_{k} \\
R_{k}=\frac{L_{k}}{\int_{S_{k}} \sigma_{k} d s_{k}}
\end{gathered}
$$

Where, $L_{k}$ is the length of the $\mathrm{k}^{\text {th }}$ solid conductor, $R_{k}$ is the conductor d.c. resistance, $\sigma_{k}$ is the conductivity of the solid conductor

We can deduce the relation between the total current $I_{k}$ and the potential difference $\nabla V_{k}$ of the $\mathrm{k}^{\text {th }}$ solid conductor

$$
\Delta V_{k}=R_{k} I_{k}+R_{k} \int_{S} \sigma \frac{\partial A}{\partial t} d s_{k}
$$

Using the Galerkin method, we can discretize (2-9) and (2-27):

$$
[S][A]-[G] \frac{d[A]}{d t}-[C][\Delta V]=[0]
$$




$$
[\Delta V]=[R][I]+[R][C]^{T} \frac{d[A]}{d t}
$$

Where $A, \Delta V$ and $I$ represent the vector potential at each node (of $\mathrm{N}$ nodes), the potential difference for each solid conductor (of $\mathrm{M}$ solid conductors) and the global current respectively. The matrices are defined by:

$$
\begin{aligned}
& S_{i j}(N \times N)=\int_{S}\left(v \nabla \lambda_{i} \nabla \lambda_{j}\right) L \mathrm{~d} s \\
& G_{i k}(N \times N)=\int_{S} \sigma \lambda_{i} \lambda_{j} L \mathrm{dS} \\
& C_{i k}(N \times M)=\int_{S} \sigma \lambda_{i} \mathrm{dS} \\
& R_{k k}(M \times M)=R_{k}
\end{aligned}
$$

Where, $R_{k}=$ the resistance of the $\mathrm{k}^{\text {th }}$ conductor

$$
\begin{aligned}
& v=\text { the reluctivity of the problem being studied } \\
& \sigma=\text { the conductivity of the problem being studied } \\
& L=\text { the length of the problem being studied }
\end{aligned}
$$

\subsubsection{Stranded conductors}

For a conductor made of thin separate $\mathrm{N}$ filaments. The filaments are connected in series. The region $\mathrm{k}$ occupied by the filaments is treated as an eddy current free material. The uniformly distributed current density given by [30-32]:

$$
J_{k}=\frac{N_{k} \cdot I_{f k}}{S_{k}}
$$

Where $I_{f k}$ is the current of a single filament. $S_{k}$ denotes the cross section of the region occupied by the filaments. 
Then Maxwell's equation then reduced to:

$$
\nabla \times((v) \nabla \times A)=\frac{N_{k} I_{f k}}{S_{k}}
$$

If $l_{k}$ is the length of the conductor (along the $\mathrm{Z}$ direction) then the potential difference between the terminals of the filamentary conductor $\Delta V$ is the sum of the voltages of all the filaments of the $\mathrm{k}^{\text {th }}$ region and given by:

A relation between the conductors total current $I_{k}$ and the voltage difference $\Delta V_{k}$ is obtained from (2-17), applied to each filament:

$$
\Delta V_{k}=\sum_{i=1}^{N_{k}}\left(R_{i} I_{f k}+\frac{N_{k} l_{k}}{S_{k}} \int_{S_{i}} \frac{\partial A}{\partial t} d s_{i}\right)
$$

Where, $V$ is the electric scalar potential and $R_{k}$ is the total resistance of the filamentary conductor. $S_{i}$ is the cross sectional area of the filament

$$
S_{i}=\frac{\lambda S_{k}}{N_{k}}
$$

Where $\lambda$ is the filling coefficient

$$
R_{i}=\frac{L_{k}}{\int_{S_{i}} \sigma d s_{i}}
$$

The value of the $\Delta V_{k}$, represents the sum of the potential difference in each filament of the $\mathrm{k}^{\text {th }}$ region. If the coil is modeled with two regions, the potential difference at the end of coil will be given by the sum of the potential difference on these two regions. If the value of $\sigma$ is constant on a filament we can write: 


$$
\Delta V_{k}=\sum_{i=1}^{N_{k}}\left(\frac{L}{\left(\sigma_{i} S_{i}\right) I_{k}}+\int_{S_{i}} \frac{L_{k}}{S_{i}} \frac{\partial A}{\partial t} d s_{i}\right)
$$

We notice that:

$$
\begin{aligned}
& \sum \frac{L}{\sigma_{i} S_{i}}=\left(\frac{L N S}{\lambda S_{k}}\right) \sum \frac{1}{\sigma_{i}} \approx\left(\frac{L N S}{\lambda S_{k}}\right) \frac{N S}{S_{k}} \int_{S_{k}} \frac{1}{\sigma} d S_{k} \\
& \sum \int_{S_{i}} \frac{L}{S_{i}} \frac{\partial A}{\partial t} d s_{i} \approx \frac{L N S}{S_{k}} \int_{S_{k}} \frac{\partial A}{\partial t} d s_{k}
\end{aligned}
$$

Finally we have,

$$
\begin{aligned}
& \Delta V_{k}=R_{k}^{\prime} I_{k}+L_{k} \frac{N S_{k}}{S_{k}} \int_{S_{i}} \frac{\partial A}{\partial t} d s_{k} \\
& R_{k}^{\prime}=\left(\frac{1}{\lambda_{k}}\right) L_{k}\left(\frac{N_{k}^{2}}{S_{k}^{2}}\right) \int_{S_{i}} \frac{1}{\sigma} d s_{k}
\end{aligned}
$$

Using the Galerkin method, we can discretize (2-35) and (2-42):

$$
\begin{aligned}
& {[S][A]=[C][I]} \\
& {[\Delta V]=\left[R^{\prime}\right][I]+[C]^{T} \frac{d[A]}{d t}}
\end{aligned}
$$

We use the same notation as previously. The matrices are defined by:

$$
\begin{aligned}
& S_{i j}(N \times N)=\int_{S}\left(v \nabla \lambda_{i} \nabla \lambda_{j}\right) L \mathrm{~d} s \\
& C_{i k}(N \times F)=-\left(\frac{N_{k} L}{S_{k}}\right) \int_{S} \sigma \lambda_{i} \mathrm{dS} \\
& R_{k k}^{\prime}(F \times F)=R_{k}
\end{aligned}
$$

Where, $R_{k}=$ the resistance of the $\mathrm{k}^{\text {th }}$ conductor

$$
N_{k}=\text { the number of wires }
$$


$F=$ the number of stranded conductors

\subsubsection{Coupling with solid conductors}

We have to put (2-29) in (2-17) to take into account the solid conductor in the electric circuit. For simplicity we considered a simple resistance-inductance circuit, so only the first and second term are considered from (2-17). Due to memory and speed we have chosen to keep the potential difference $\Delta V$ as unknown. The system to solve is:

$$
\begin{aligned}
& {[S][A]-[G] \frac{d[A]}{d t}-[C][\Delta V]=[0]} \\
& {[R][C]^{T} \frac{d[A]}{d t}-[\Delta V]+[R][I]=[0]} \\
& {[D][\Delta V]+\left[Z_{m}^{\prime}\right]\left[I_{m}\right]+\left[L_{m}\right] \frac{d\left[I_{m}\right]}{d t}=\left[E_{m}\right]}
\end{aligned}
$$

Where the matrix $[D]$ is defined by the relation:

$$
[I]=[D]^{T}\left[I_{m}\right]
$$

The value of $D_{i j}(K \times M)$ becomes

+1 , if conductor $i$ belongs to mesh $\mathrm{j}$ with right way

-1 , if conductor $i$ belongs to mesh $j$ with wrong way

0 , if conductor $i$ does not belong to mesh $j$

$\left[Z_{m}^{\prime}\right]$ is the matrix of impedances

$Z_{i i}^{\prime}$ is the addition of impedances in the electric mesh $i$ without the resistances of solid conductors.

The system comprising of (2-49), (2-50), (2-51) can be symmetrical: 


$$
\begin{aligned}
& {[S][A]-[G] \frac{d[A]}{d t}-[C][\Delta V]=[0]} \\
& -[C]^{T} \frac{d[A]}{d t}+\left[R^{-1}\right][\Delta V]-[D]^{T}\left[I_{m}\right]=[0] \\
& -[D][\Delta V]-\left[Z_{m}^{\prime}\right]\left[I_{m}\right]-\left[L_{m}\right] \frac{d\left[I_{m}\right]}{d t}=-\left[E_{m}\right]
\end{aligned}
$$

The matrix $[R]$ is diagonal and strictly positive by definition.

\subsubsection{Coupling with stranded conductors}

The same method is used as before. We obtain:

$$
\begin{aligned}
& {[S][A]-\left[C^{\prime}\right][I]=[0]} \\
& {\left[E_{m}\right]=\left[Z_{m}\right]\left[I_{m}\right]+\left[L_{m}\right] \frac{d\left[I_{m}\right]}{d t}+\left[D^{\prime}\right][C]^{T} \frac{d[A]}{d t}}
\end{aligned}
$$

As previously we have:

$$
[I]=\left[D^{\prime}\right]^{T}\left[I_{m}\right]
$$

This system of equations can be symmetrical:

$$
\begin{aligned}
& {[S][A]-\left[C^{\prime}\right]\left[D^{\prime}\right]^{T}\left[I_{m}\right]=[0]} \\
& -\left[D^{\prime}\right]\left[C^{\prime}\right]^{T} \frac{d[A]}{d t}-\left[Z_{m}\right]\left[I_{m}\right]-\left[L_{m}\right] \frac{d\left[I_{m}\right]}{d t}=-\left[E_{m}\right]
\end{aligned}
$$

The system of (2-56) and (2-57) can be solved simultaneously with the system of equations (2-59) and (2-60). We obtain:

$$
\begin{aligned}
& {[S][A]-[G] \frac{d[A]}{d t}-[C][\Delta V]-\left[C^{\prime}\right]\left[D^{\prime}\right]^{T}\left[I_{m}\right]=[0]} \\
& -[C]^{T} \frac{d[A]}{d t}+\left[R^{-1}\right][\Delta V]-[D]^{T}\left[I_{m}\right]=[0]
\end{aligned}
$$




$$
-\left[D^{\prime}\right]\left[C^{\prime}\right]^{T} \frac{d[A]}{d t}-[D][\Delta V]-\left[Z_{m}^{\prime}\right]\left[I_{m}\right]-\left[L_{m}\right] \frac{d I_{m}}{d t}=-\left[E_{m}\right]
$$

The equation actually solved depends on the application and the results needed.

\subsection{Coupling of FE model with Simulink}

FE is coupled with Simulink to perform integrated system simulations that utilize Simulink models library through an S-function functional block [181]. The S-function functional block schematic is shown in Fig.2.2. A constant time step $\Delta t$ is used for the coupled FEM computation.

\section{Simulation Time}

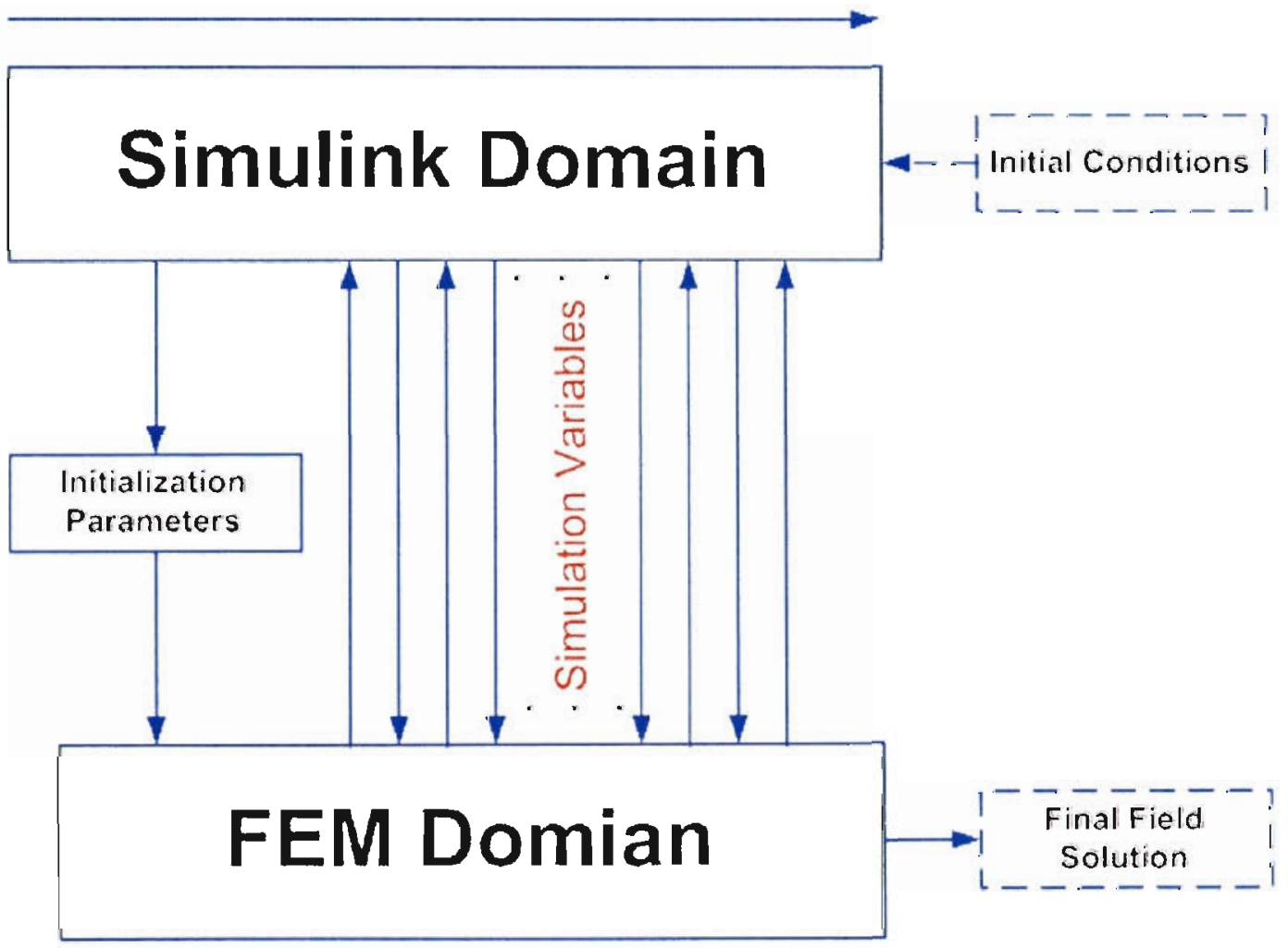

Fig.2.2 Schematic of the coupling between the FEM and SIMULINK

The sampling time of the FEM block is set to that value, and the same time step sizes are used elsewhere in the model. The input and output variables are defined as stated 
above and are updated discretely at the major steps as set for the finite element analysis. The average of the input voltage from two adjacent steps is replaced by a single value taken from the middle point between the steps.

To couple FE with Simulink first, the initialization parameters are stored in a file, from which the FEM reads the parameters before the first time step. During the timestepping simulation, the simulation variables are passed directly back and forth between the FEM block and Simulink. After the simulation, the field solution is stored in a file with the same format as the one with the initial field while the simulation variables are stored in MATLAB's [181].

\section{2-8 Operational parameters extraction}

This section describes the parameter extraction method from the physical FE geometry. This includes the inductance calculation, resistance calculation, and capacitances calculation for certain geometry.

\subsubsection{Inductance calculation for high and low frequency studies}

The inductance is complicated functions of the conductor's currents, saturation characteristics of the electromagnetic device, the supply frequency, and the displacement between stationary and rotating windings. Methods for computing the inductance using FEM computation in conjunction with energy perturbation method $(E / C)$ techniques is outlined in this section. The energy perturbation method is used to compute both the self and the mutual inductance. The method can take into the consideration the effect of the electromagnetic device geometry and loading condition on the inductance. The method detail is as follows: 
For a lossless (conservative) magnetic field with $\mathrm{n}$ coupled conductors, the instantaneous terminal power of the $j^{\text {th }}$ conductor can be written as:

$$
P_{j}=R_{j} i_{j}^{2}+i_{j} L_{j 1} \frac{d i_{1}}{d t}+i_{j} L_{j 2} \frac{d i_{2}}{d t}+\ldots \ldots . .+i_{j} L_{j n} \frac{d i_{n}}{d t}
$$

Where: $V_{j}:$ terminal voltage of conductor $\mathrm{j}$,

$i_{j}:$ Current in conductor $\mathrm{j}$,

$\lambda_{j}:$ Total flux linkage,

$L_{j k}:$ Incremental inductance which is the partial derivative of flux linkage $\partial \lambda_{j}$ with respect to current $i_{k}$ where $\mathrm{k}=1,2,3 \ldots \mathrm{n}$.

The first term represents the instantaneous power dissipated, and the remaining terms represent the instantaneous reactive power associated with the flux linkage with the $j^{\text {th }}$ coil.

The total energy $\mathrm{W}$ of the system,

$$
W=\sum_{j-1}^{n} W_{j}
$$

Where:

$$
W_{j}=\sum_{k=1}^{n} \int_{i_{k}(0)}^{i_{k}(t)}\left(L_{j k} i_{j}\right) d i_{k}
$$

If we disturb the $\mathrm{n}$ currents by small increments, $\Delta i_{1}, \Delta i_{2},-, \Delta i_{n}, L_{j k}$ remain constant, so the energy increment $\Delta w$ is given by:

$$
\Delta w=\sum_{j=1}^{n} \sum_{k=1}^{n} L_{j k} \int_{i_{k}}^{i_{k}+\Delta i_{k}} i_{j} d i_{k}
$$


Performing the integration, and using difference equation to express the partial derivatives of the global energy with respect to the incremental change in currents. The inductances can be found by using the following equations.

$$
\begin{aligned}
& L_{j j}=\frac{\left[w\left(i_{j}+\Delta i_{j}\right)-2 w\left(i_{j}\right)+w\left(i_{j}-\Delta i_{j}\right)\right]}{\left(\Delta i_{j}\right)^{2}} \\
& \begin{aligned}
\frac{\left(L_{j k}+L_{k j}\right)}{2}=[ & w\left(i_{j}+\Delta i_{j}, i_{k}+\Delta i_{k}\right)-w\left(i_{j}-\Delta i_{j}, i_{k}+\Delta i_{k}\right) \\
& \left.-w\left(i_{j}+\Delta i_{j}, i_{k}-\Delta i_{k}\right)+w\left(i_{j}-\Delta i_{j}, i_{k}-\Delta i_{k}\right)\right] /\left(4 \cdot \Delta i_{j} \Delta i_{k}\right)
\end{aligned}
\end{aligned}
$$

Where,

$$
w\left(i_{j} \pm \Delta i_{j}, i_{k} \pm \Delta i_{k}\right)=w\left(i_{j}, i_{k}\right)+\Delta w\left( \pm \Delta i_{j}, \pm \Delta i_{k}\right)
$$

For an electromagnetic device that consists of individual conductor elements. The self inductances of each segment and mutual inductances between each other are calculated from FE. With this information we can find the total self and mutual inductances of the whole electromagnetic device.

The self inductance of the winding is the sum of all self inductances $\left(L_{i}\right)$ of conductors in this winding plus the sum of the mutual inductances $\left(L_{i k}\right)$ between the conductors within one winding. The complete self inductance of the windings can be expressed as:

$$
L=\sum_{i=1}^{n} L_{i}+2 \sum_{i=1}^{n-1} \sum_{k=i+1}^{n} L_{i k}
$$

Where i, $\mathrm{k}$ are the numbers of the individual conductors. It should be mentioned, that $L_{i k}$ sign depends on the current direction in the segment. 
The mutual inductance $L_{A B}$ between two windings (A and $\mathrm{B}$ ) is the sum of the mutual inductances of each conductor in winding A to each conductor of winding B.

The complete mutual inductance of the windings can be expressed as:

$$
L_{A B}=\sum_{i=1}^{n_{A}} \sum_{k=n_{A}+1}^{n_{A}+n_{R}} L_{i k}
$$

Where $n_{A}$ and $n_{B}$ are the numbers conductors in windings $\mathrm{A}$ and $\mathrm{B}$ respectively. i, $\mathrm{k}$ are the numbers of the individual conductors.

\subsubsection{Resistance calculation}

The ohmic resistance of the winding is caused by finite conductivity of the metal. An analytic estimation of the series resistance may be obtained from the basic resistance formula.

$$
R=\frac{L}{\sigma A}
$$

Where $\sigma$ represents the conductivity of the material, $\mathrm{L}$ is the total length of the winding, and $\mathrm{A}$ is the conductor cross sectional area. Another way to find the resistance is by using DC test. This value can only be used for direct current and low frequencies studies. The skin effect on the resistance value can be obtained by using closed formulas for different conductor shapes or by using FEM [36].

With the increase in the supply frequency the current density becomes more and more nonuniform due to skin and proximity effect in the metal. This origin of theses effects is due to the penetration of time varying magnetic fields in the metal. In skin effect the magnetic field in the metal is produced by the conductor current itself. The induced eddy currents in the conductor force most of the current to flow near the metal conductor 
boundary. This will cause an increase in the winding resistance with the increase in the supply frequency.

In contrast to the skin effect, the proximity effect is due to the effect of other conductors magnetic fields on the current flow in the conductor. This effect will cause non-uniform current distribution in the conductor. Which like the skin effect will reduce the effective cross-sectional area of the conductor which in return will increase the resistance. The complexity of the mathematical problem is significantly increased with the increase in number of conductors. It is not possible to find the resistance with a closed formula as it is possible for the skin effect. Therefore numerical methods must be used.

In order to mesh the conductor's region for the high frequency problem, the size of the mesh elements used should be smaller than the skin depth.

\subsubsection{Capacitance calculation}

The shunt- or parallel capacitances (capacitance between windings or from winding to ground) can be calculated either by using traditional analytical methods or by using computer methods such as the electrostatic FEM. The analytical approach ignores the field non linear effects. The electrostatic problem is solved for the calculation of capacitances as capacitances are a function of geometry and material parameters rather than frequency. An electrostatic analysis is assumed to be a linear analysis that means the electric field is proportional to the applied voltage. The analysis determines the electric scalar potential distribution caused by applied voltage. The turns were treated as perfect conductors and hence are not meshed from inside. Boundaries of turns as well as insulation surrounding the turn are finely meshed. The capacitance calculation is based on the energy principle. By applying voltage on turns, the ground capacitance matrix is 
calculated from the stored static energy. Following Maxwell equation is solved during electrostatic analysis.

$$
\nabla \bullet(\varepsilon \nabla V)=-\rho
$$

Where, $\rho$ is surface charge density, $\varepsilon$ is dielectric permittivity, $\mathrm{V}$ is electric scalar potential.

The series capacitance is the capacitance between different turns of the same winding and is a determinant for the electrostatic voltage distribution. In FEM electrostatic analysis the capacitance is assumed to be function of the geometry only and thus we ignore the frequency effect. FEM computes the compute the capacitance by solving for the electrostatic charge $(\mathrm{Q})$ using the equations $Q=C V$ and $\sum Q=0$.

The capacitances matrix can be expressed in term of the conductor charge $(\mathrm{Q})$ and the conductor voltage for $\mathrm{N}$-conductor system as follows:

$$
\begin{aligned}
& Q_{1}=C_{11} V_{1}+C_{12} V_{2}+\ldots+C_{1 N} V_{N} \\
& Q_{2}=C_{21} V_{1}+C_{22} V_{2}+\ldots+C_{2 N} V_{N} \\
& \ldots \\
& Q_{N}=C_{N 1} V_{1}+C_{N 2} V_{2}+\ldots+C_{N N} V_{N}
\end{aligned}
$$

Where $Q_{i}$ is conductor $i$ total charge, $V_{i}$ is the potential on conductor $i$, and $C_{i i}$ is the $i-i$ component of the capacitance matrix, $C_{i j}$ is the $i-j$ component of the capacitance matrix.

In order to obtain a capacitance matrix of order $\mathrm{N}$ we need to run $\mathrm{FE}$ electrostatic analysis $\mathrm{N}$ times using batch file. 


\section{Chapter 3: Simulation of Shipboard Power System Components Using FEM}

\subsection{Introduction}

This chapter deals with modeling of different electromagnetic power devices using coupled-FEM. This approach was described in chapter 2 . This technique will provide an efficient way to optimize, to find out the response of the different devices under different operating conditions, and to evaluate their operational parameters during the design process.

\subsection{Finite element modeling of the three phase power transformer}

\subsubsection{The FEM model}

A 3-phase, 150-kVA, 240/120 V, $60 \mathrm{~Hz}$, transformer was modeled using the coupled FE-circuit method. The transformer geometry is shown in Fig. 3.1. High voltage and low voltage windings are concentric cylinders surrounding a vertical core leg of rectangular cross section. The conductors have constant conductivities. The primary and the secondary windings are represented by rectangles of corresponding materials in the FE domain. The primary side is delta connected while the secondary is star connected. The magnetic core is an isotropic non-linear magnetic material defined by analytic saturation curve shown in Fig 3.2.

The finite element mesh of the transformer consisting of 13645 nodes forming 5083 second order elements as illustrated in Fig. 3.3.

Dirichlet boundary condition was adopted on the external circle of infinite region surrounding the transformer.

We conducted different studies to evaluate the transformer behavior under different operating conditions and to obtain the transformer operational parameters. 


\subsubsection{Three phase transformer supplying linear load}

The transformer secondary is coupled to resistive load in the circuit domain as shown in Fig. 3.4. An open circuit test is performed to obtain the magnetizing current by connecting a very high resistor to the transformer secondary the magnetizing current is shown in Fig 3.5.

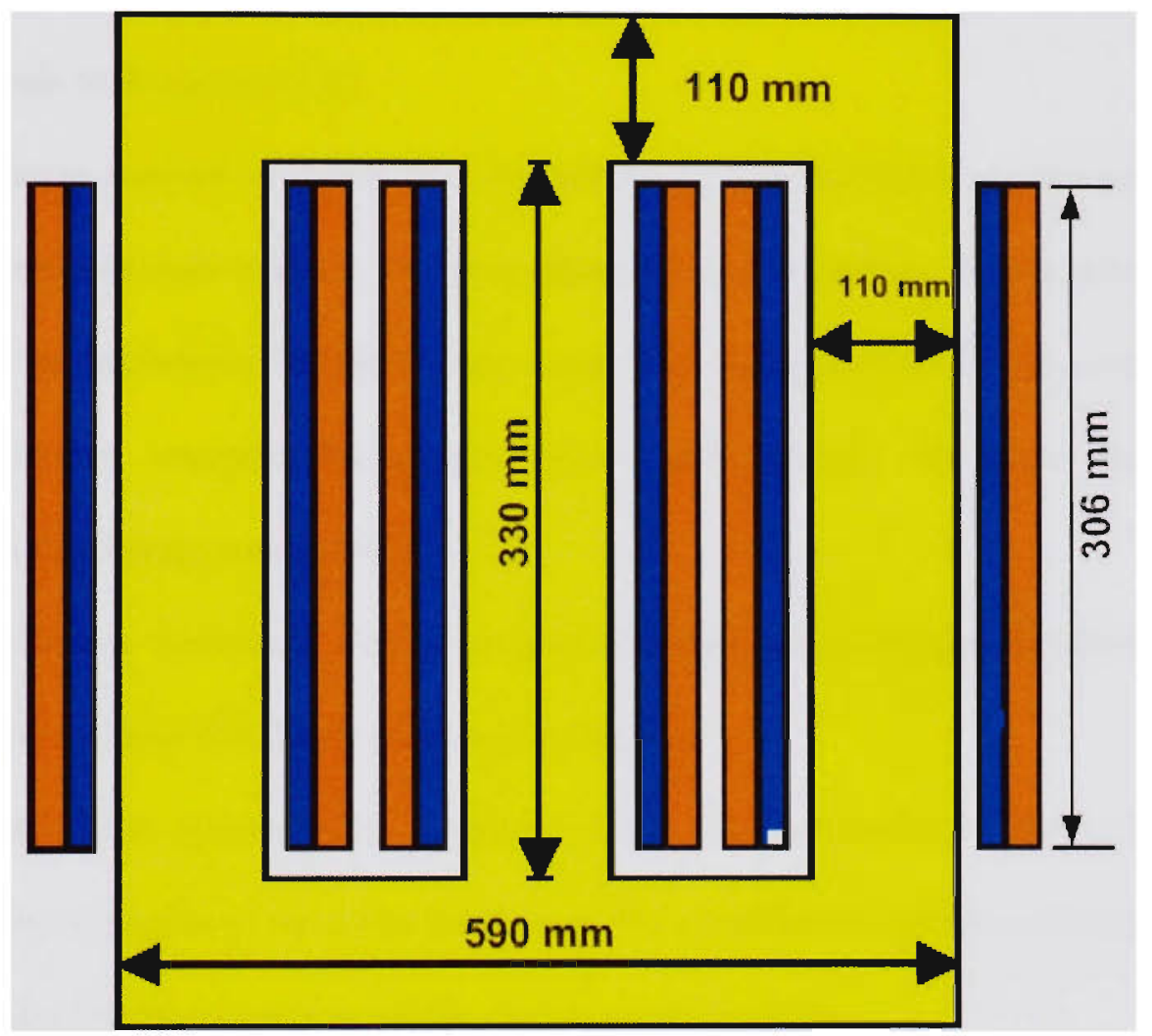

Fig.3.1 The used transformer geometry

\subsubsection{Three phase transformer supplying non-linear load}

The increase utilization of non-linear power electronics components coupled with the more prevalent use of electronic equipments has increased the harmonics generated and brought power quality issues on the distribution network to the forefront. The harmonics increase can be a source of a variety of undesirable effects in the energy system. For 
example, the harmonics can cause signal interference, over-voltages, equipment overheating, and malfunction.

Transformers are most affected with this problem and may need to be derated to as much as $50 \%$ capacity when feeding loads with extremely distorted current waveforms and reduction in their service life. The IEEE/ANSI C57.110-1998 standards states that a transformer subject to nonsinusoidal load current having more than $5 \%$ total harmonics distortion needs to be derated [53].

The common sources of harmonics and power quality problems are the non-linear power electronic devices such as the three-phase inverter supplying motor drives. This increase in the harmonics behavior may cause the maloperation of the control and protective devices, increase the system losses, and increase the noise and EMC generation in the energy system [97].

The transformer secondary side is coupled to a three phase bridge rectifier feeding resistive-inductive load ( $\mathrm{RL}$ load) as shown in Fig. 3.6.

Usually in industrial networks the strengths of the odd harmonics of the voltage are higher than the strengths of the even harmonics. We conducted a number of studies with wide variation of harmonic characteristics in the supply voltage.

We distorted the supply voltage with odd harmonics up to the 15 th component. For each harmonic component added to the voltage waveform, there are two cases; peaking case and flattened peak case. In the peaking case, the positive peak of the harmonic waveform coincides with the positive fundamental peak. In the flattened peak case, the negative peak of the harmonic component coincides with the positive fundamental peak. 


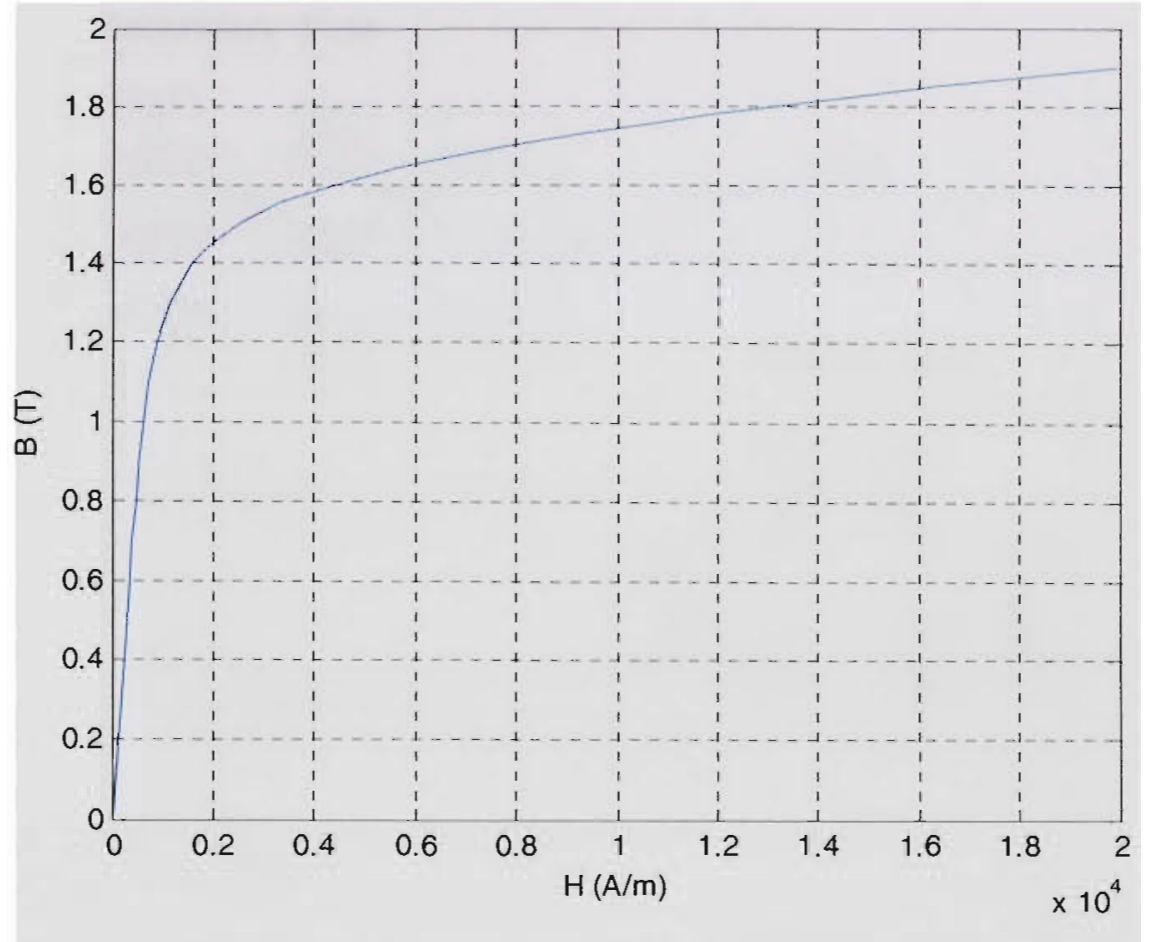

Fig.3.2 Transformer core magnetizing curve

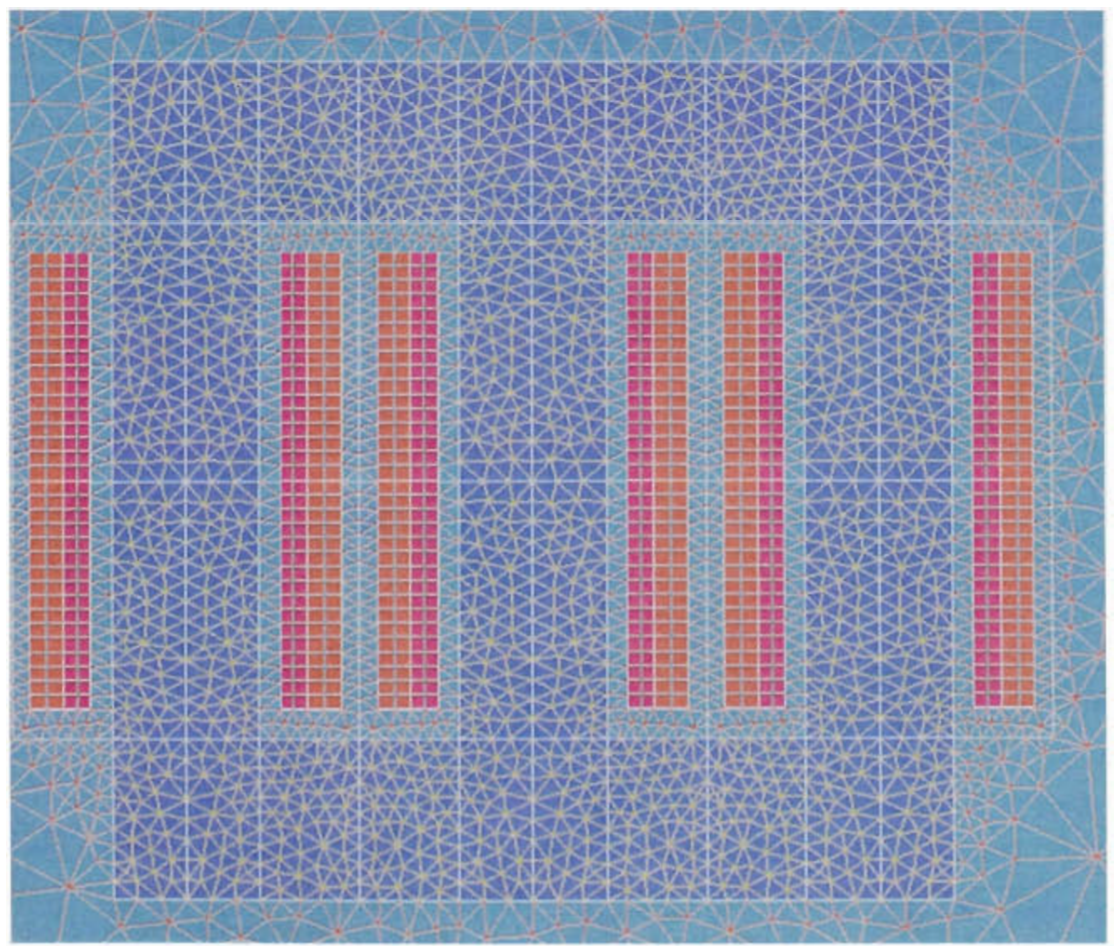

Fig.3.3 Transformer finite element mesh details 


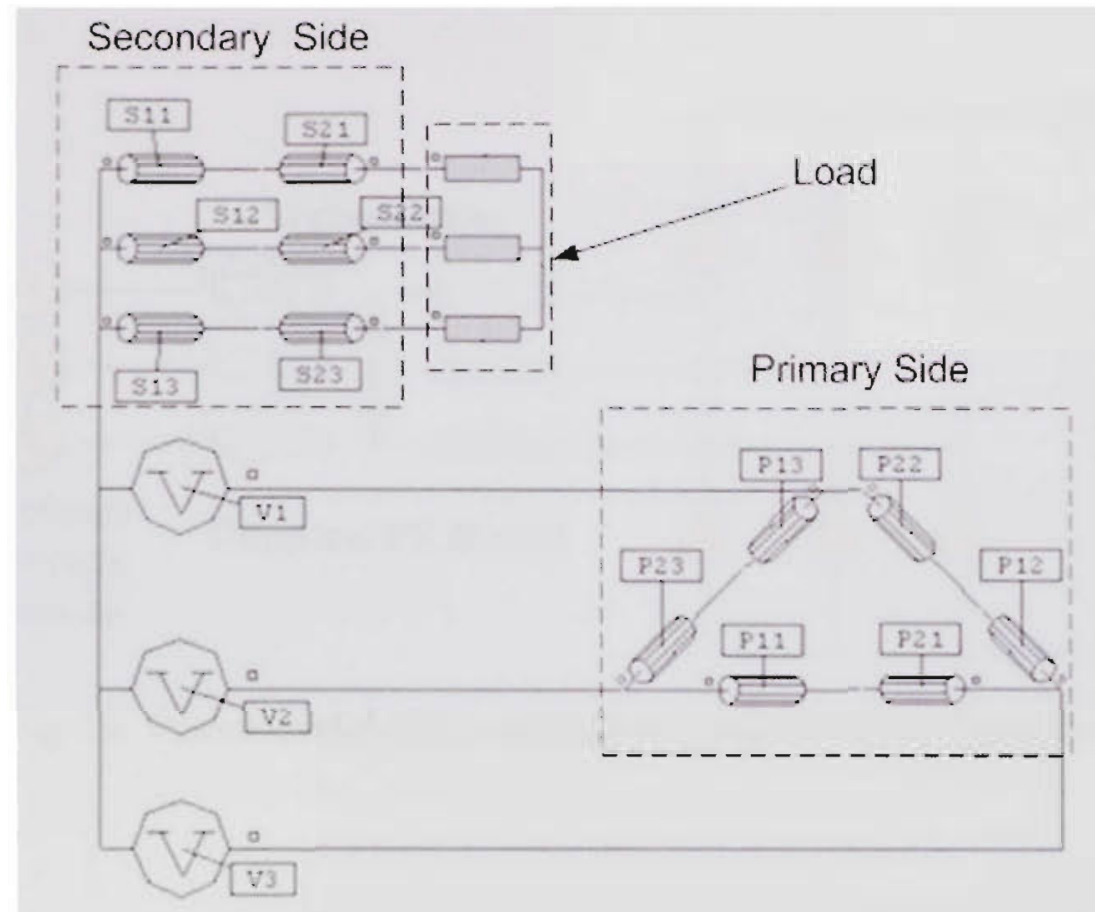

Fig.3.4 Transformer circuit domain representation

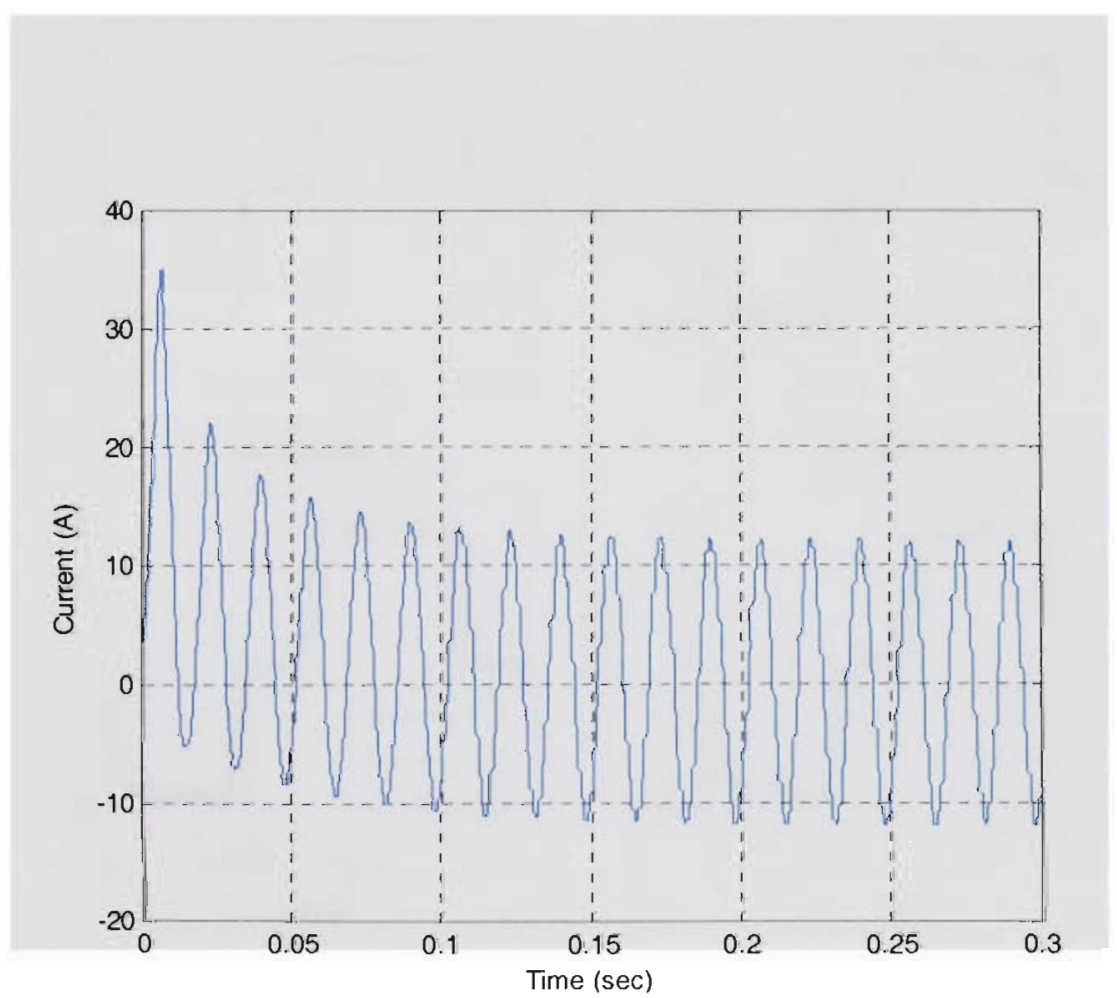

Fig.3.5 Transformer magnetizing current obtained from FE 


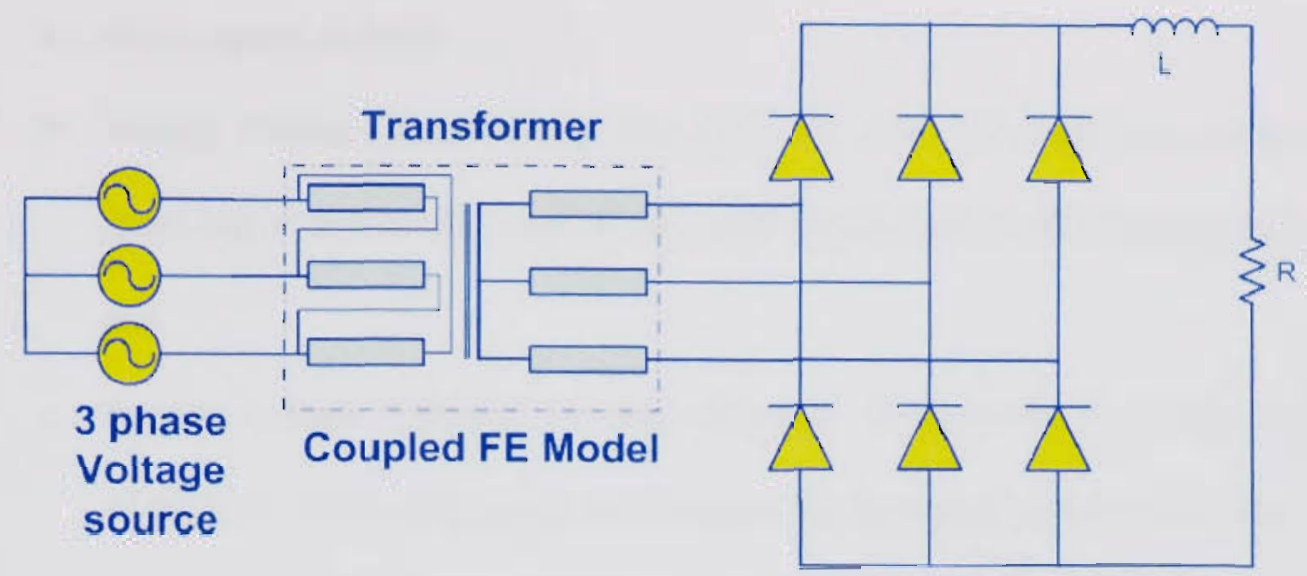

Fig.3.6. Circuit model of the transformer, source and nonlinear load
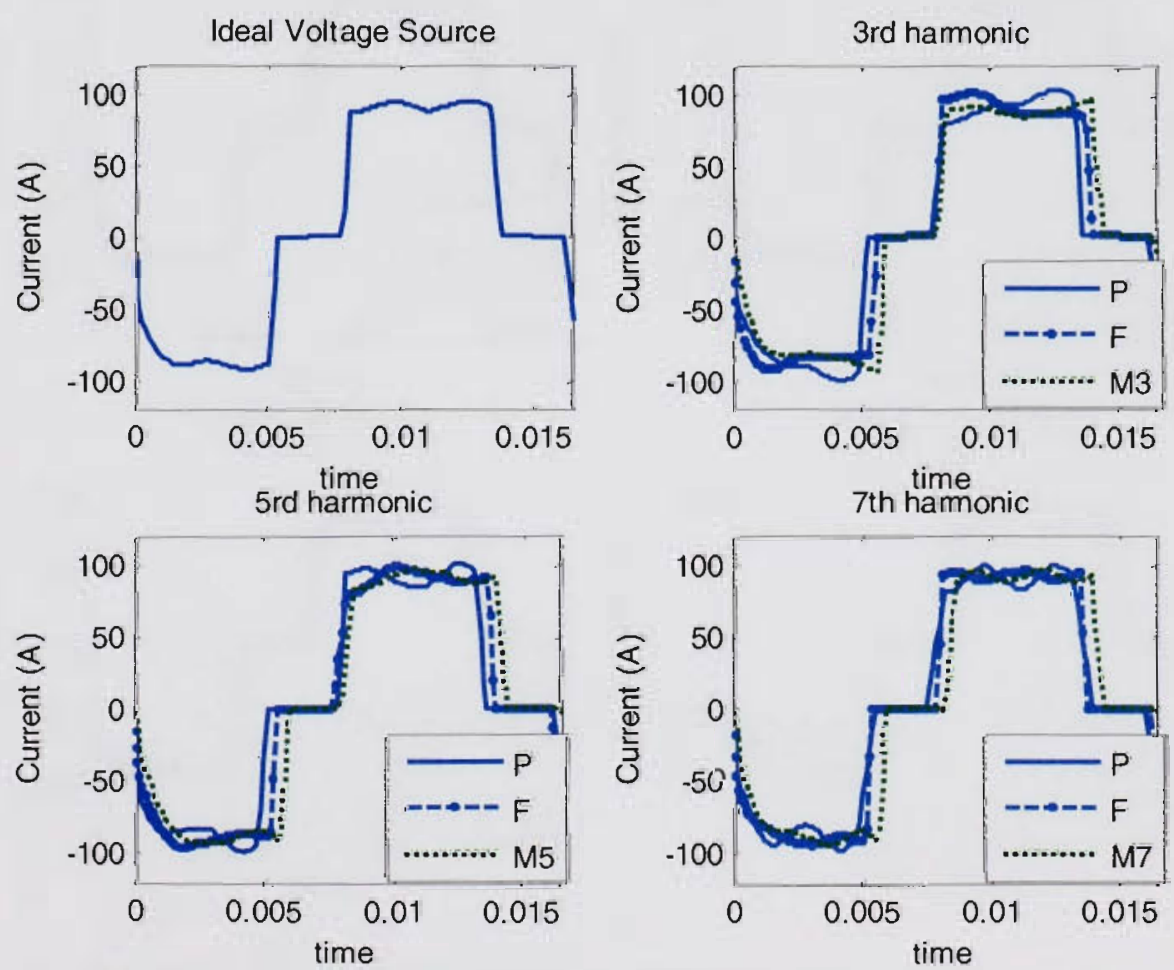

Fig.3.7. Transformer secondary current for ideal voltage supply and voltage supply distorted by $3^{\text {rd }}, 5^{\text {th }}$, and $7^{\text {th }}$ harmonics 
The study includes the following scenarios:

a. Ideal supply voltage,

b. Supply voltage distorted by the $3 \mathrm{rd}$, 5th, and 7 th harmonic components (peaking or flattened), respectively with a peak value $10 \%$ fundamental peak, and

c. Supply voltage distorted by odd harmonic components (3rd, 5th, 7th, 9th, 11 th, 13th, 15th) combined, with one of the harmonic component $10 \%$ of the fundamental peak and the other with different lower percentage (between $0.1 \%-3 \%$ of the fundamental).
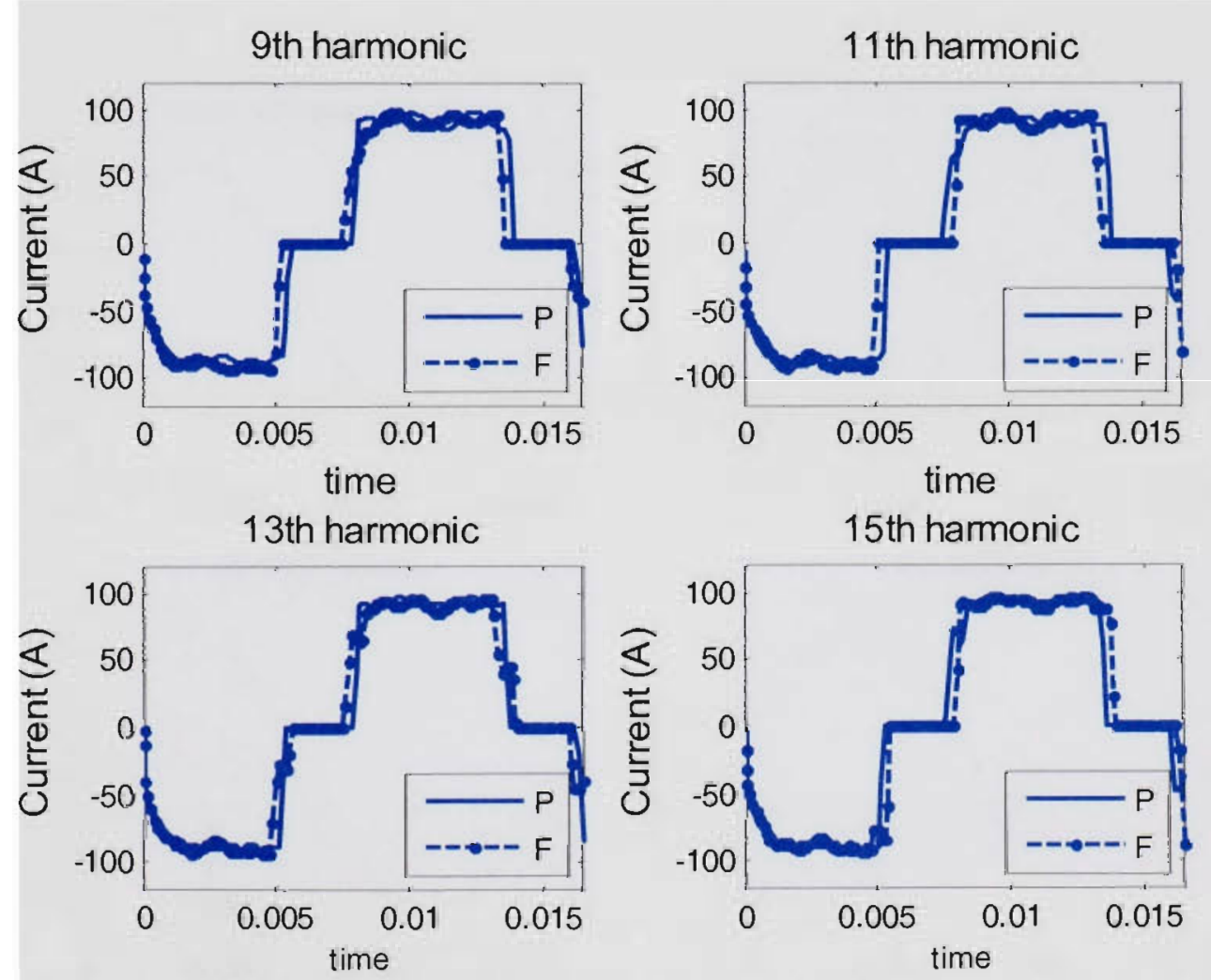

Fig.3.8. Transformer secondary current for supply distorted by $9^{\text {th }}, 11^{\text {th }}, 13^{\text {th }}$ and $15^{\text {th }}$ harmonics 
Fig. 3.7 and Fig. 3.8 shows transformer phase A secondary current obtained from FE under different voltage supply harmonics.

Fig. 3.9 shows the magnetizing current under different voltage supply harmonics. Fig. 3.10 and 3.11 shows the converter DC side current under different voltage supply harmonics.

Here ' $\mathrm{Mx}$ ' stands for a waveform that contains a number of harmonics with ' $\mathrm{x}$ ' as the highest harmonic component ( $10 \%$ of the fundamental). And, ' $\mathrm{P}$ ' and ' $\mathrm{F}$ ' stand for peaking case and flattened case respectively.

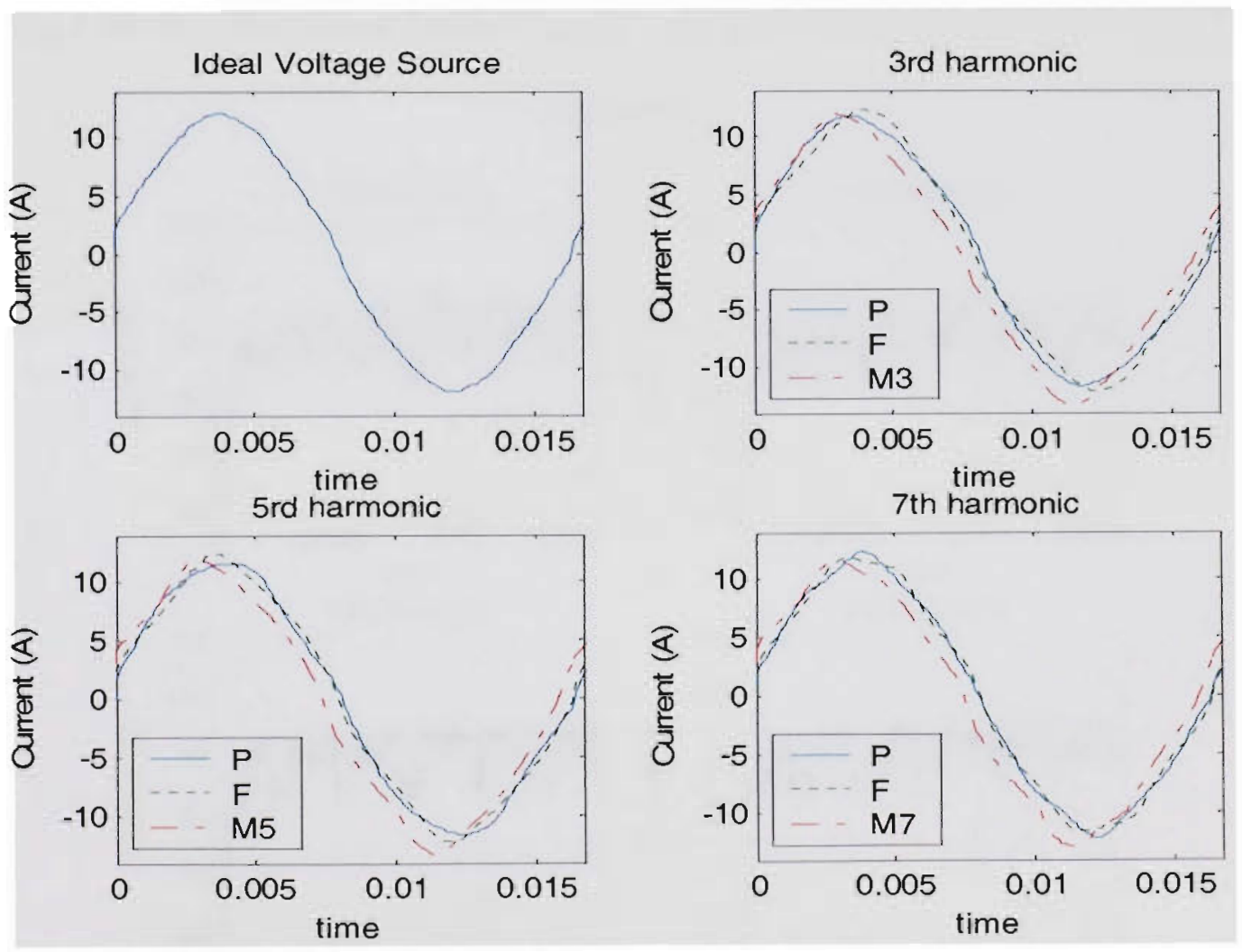

Fig.3.9. Transformer secondary current for different supply harmonics 

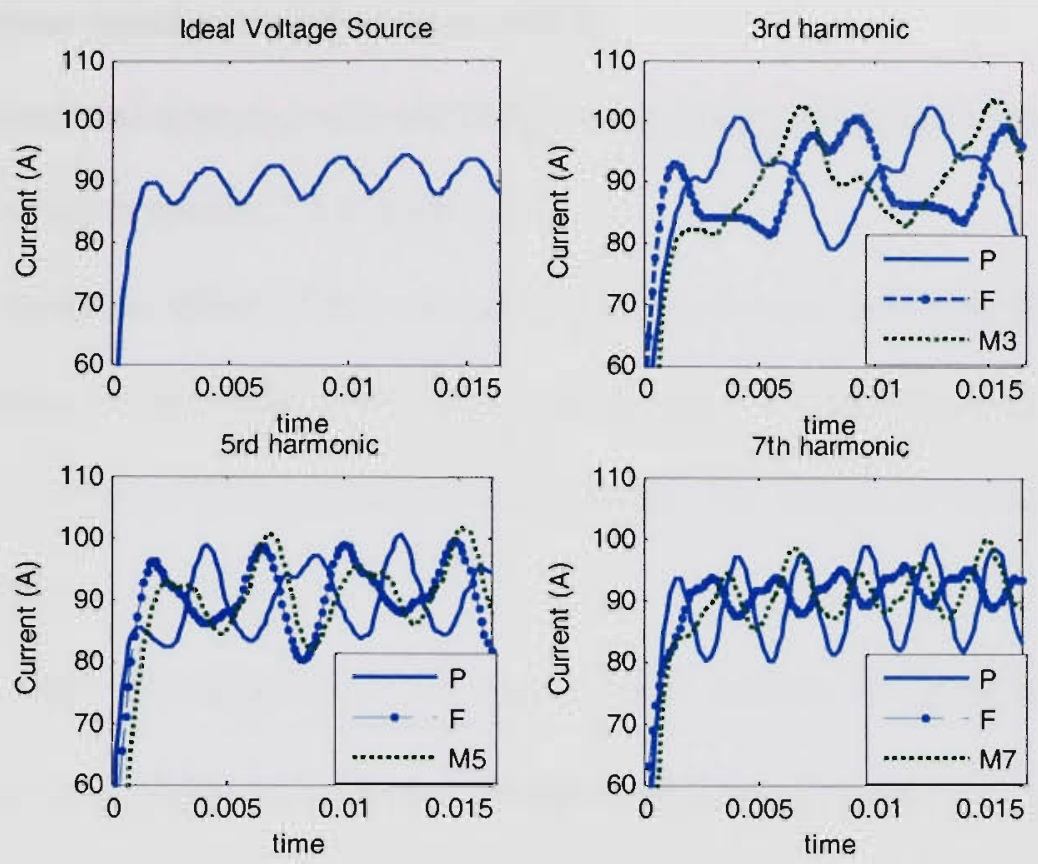

Fig.3.10. DC side current for ideal supply and supply distorted by $3^{\text {rd }}, 5^{\text {th }}$, and $7^{\text {th }}$ harmonics
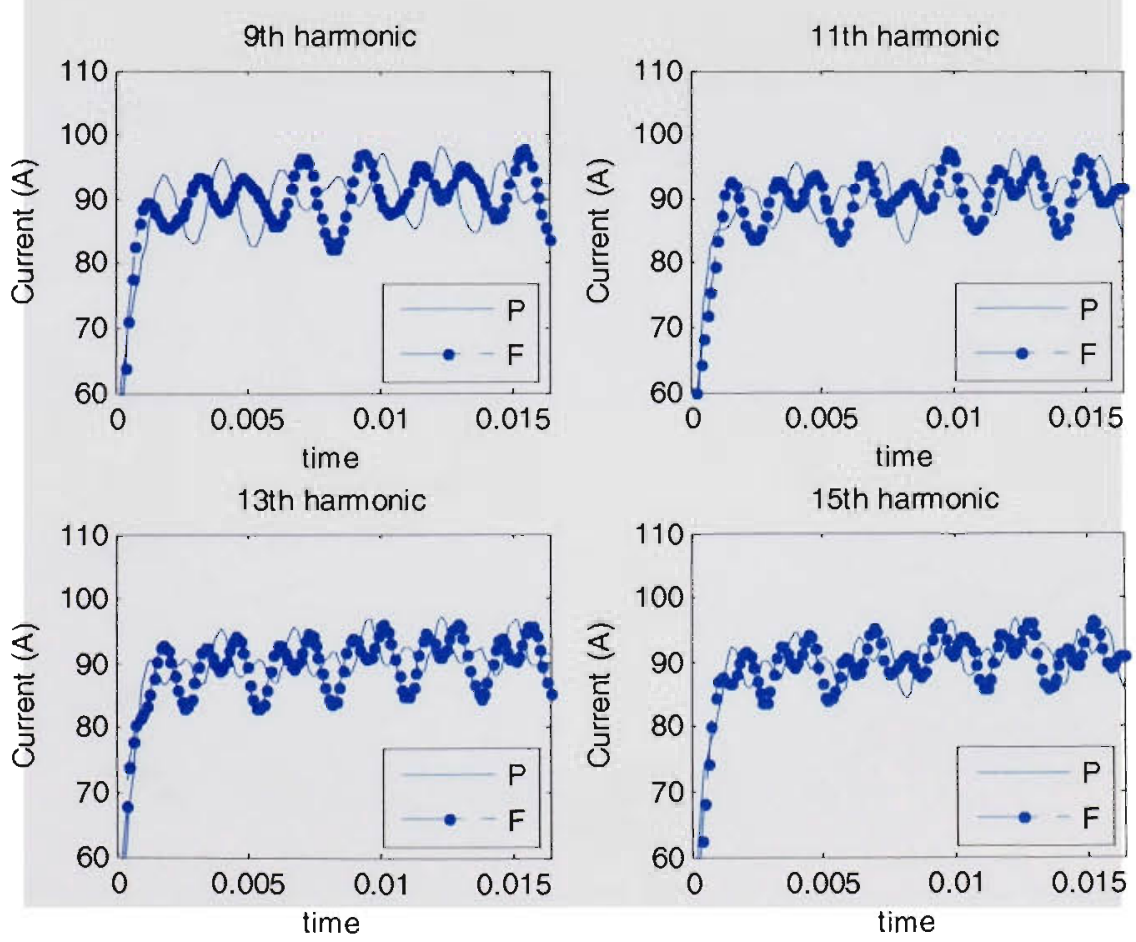

Fig. 3.11. DC side current for supply distorted by $9^{\text {th }}, 11^{\text {th }}, 13^{\text {th }}$ and $15^{\text {th }}$ harmonics 


\subsubsection{Three phase transformer inductances profile}

The transformer inductances were calculated using energy perturbation method $(E / C)$ techniques described in section 2.8.1 in chapter 2.

In order to study the effect of the saturation level on the self and mutual inductances of the six windings of the transformer, these inductances were calculated at $25 \%, 50 \%$, $75 \%, 100 \%$, and $125 \%$ of the rated magnetizing current. The results of this study give 36 sets of inductances.

Since $M_{j k}=M_{k j}$ the inductances we have to find will be 21 inductances ( 6 self inductances plus 15 mutual inductances). These inductances are listed in (3-1). Figure 3.12 through Fig. 3.32 shows the transformer primary inductances, secondary inductances and the mutual inductance as a function of the magnetizing current over a complete cycle.

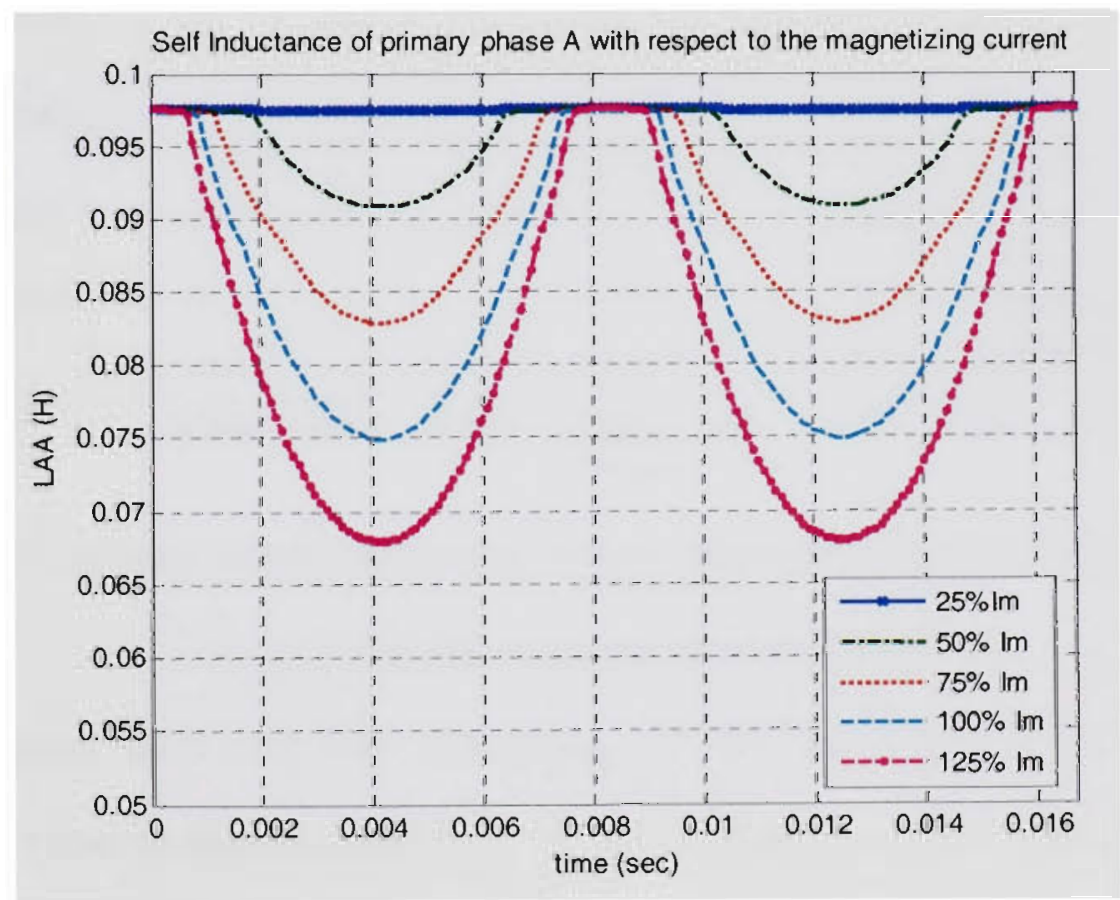

Fig.3.12. Transformer phase a primary inductance as a function of the magnetizing current 


$$
L=\left[\begin{array}{llllll}
L_{A A} & L_{A B} & L_{A C} & L_{A a} & L_{A b} & L_{A c} \\
L_{A B} & L_{B B} & L_{B C} & L_{B a} & L_{B b} & L_{B c} \\
L_{A C} & L_{B C} & L_{C C} & L_{C a} & L_{C b} & L_{C C} \\
L_{A a} & L_{B a} & L_{C a} & L_{a a} & L_{a b} & L_{a c} \\
L_{A b} & L_{B b} & L_{C b} & L_{a b} & L_{b b} & L_{b c} \\
L_{A c} & L_{B c} & L_{C c} & L_{a c} & L_{b c} & L_{c c}
\end{array}\right]
$$

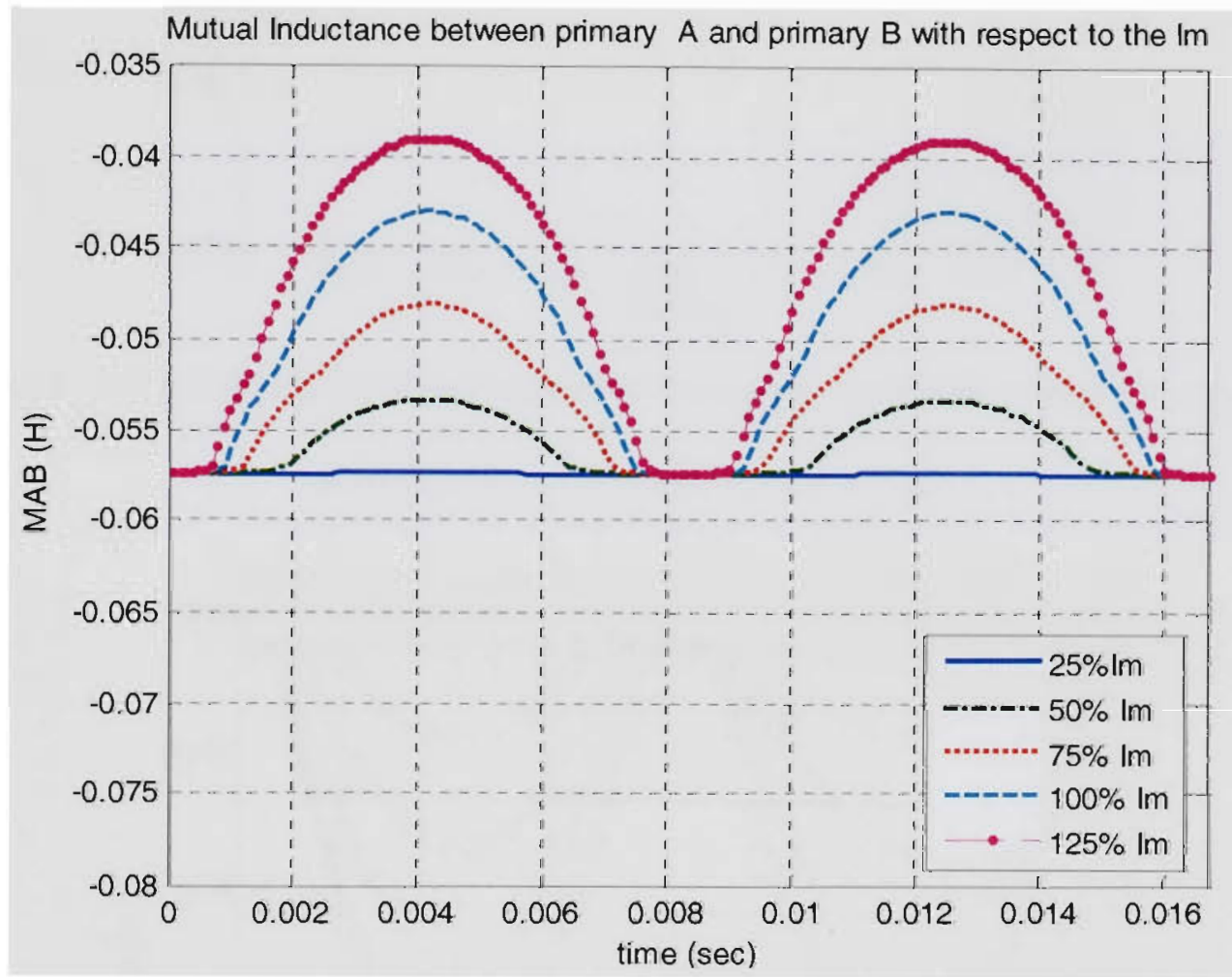

Fig.3.13. Transformer mutual inductance between phase a primary and phase b primary side as a function of the magnetizing current

The Figures show the high dependency of the transformer self and mutual inductances value on the core saturation. The inductance has its lowest value when the core is most saturated and its highest value when the core saturation is at its lowest level. These inductance will be used later to build the phase variable model. 


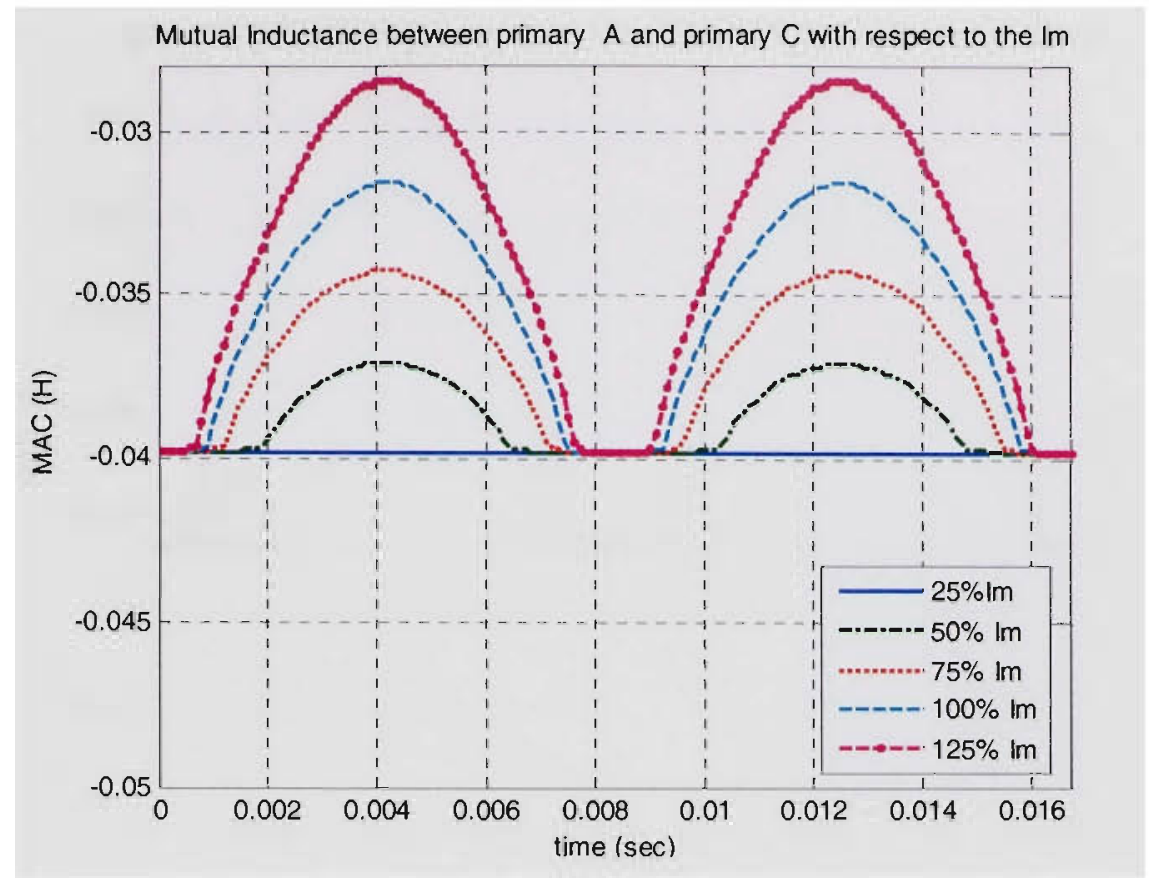

Fig.3.14. Transformer mutual inductance between phase a primary and phase c primary as a function of the magnetizing current

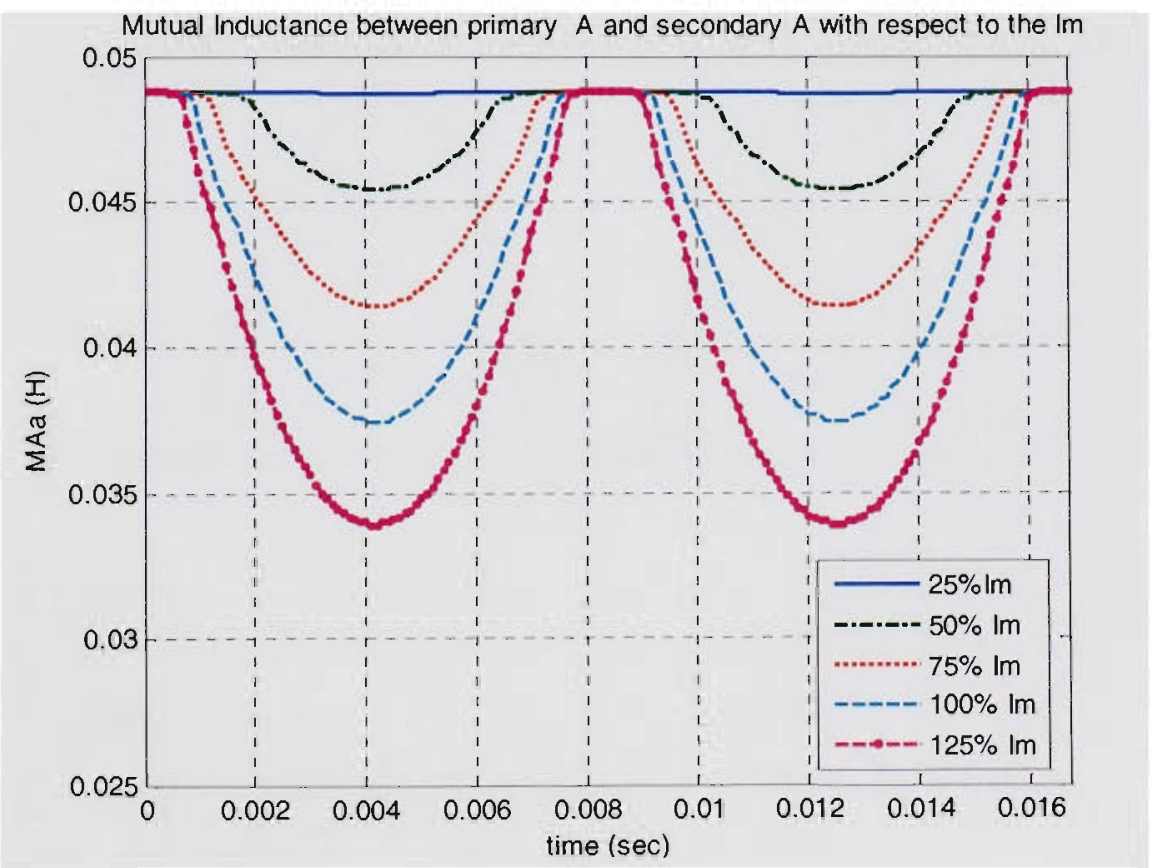

Fig.3.15. Transformer mutual inductance between phase a primary and phase a secondary side as a function of the magnetizing current 


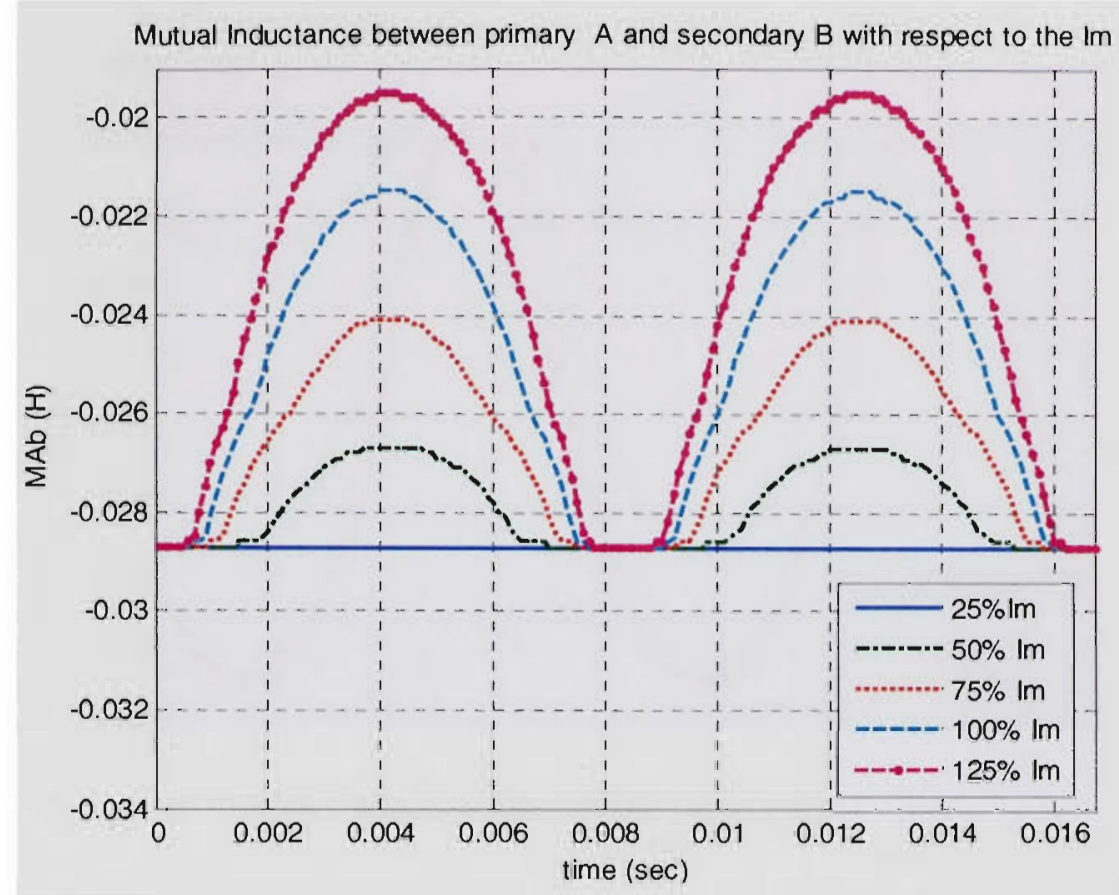

Fig.3.16. Transformer mutual inductance between phase a primary and phase $b$ secondary side as a function of the magnetizing current

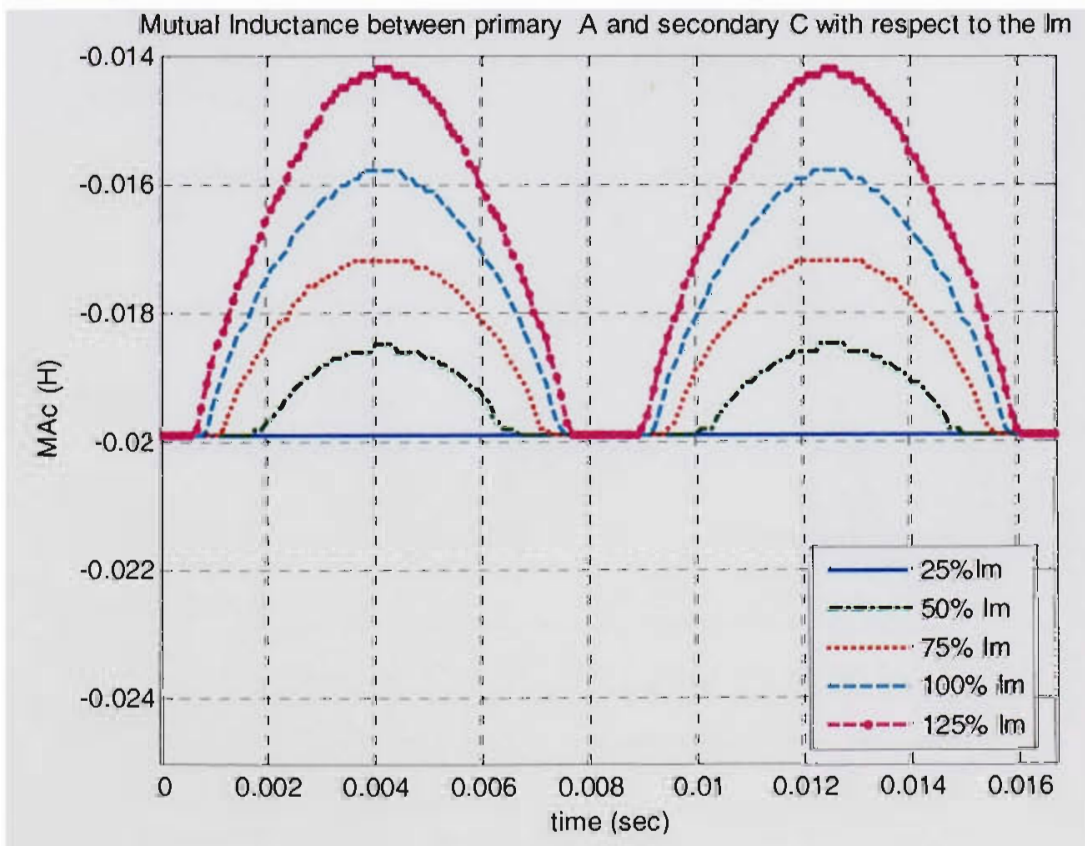

Fig.3.17. Transformer mutual inductance between phase a primary and phase $\mathrm{c}$ secondary side as a function of the magnetizing current 


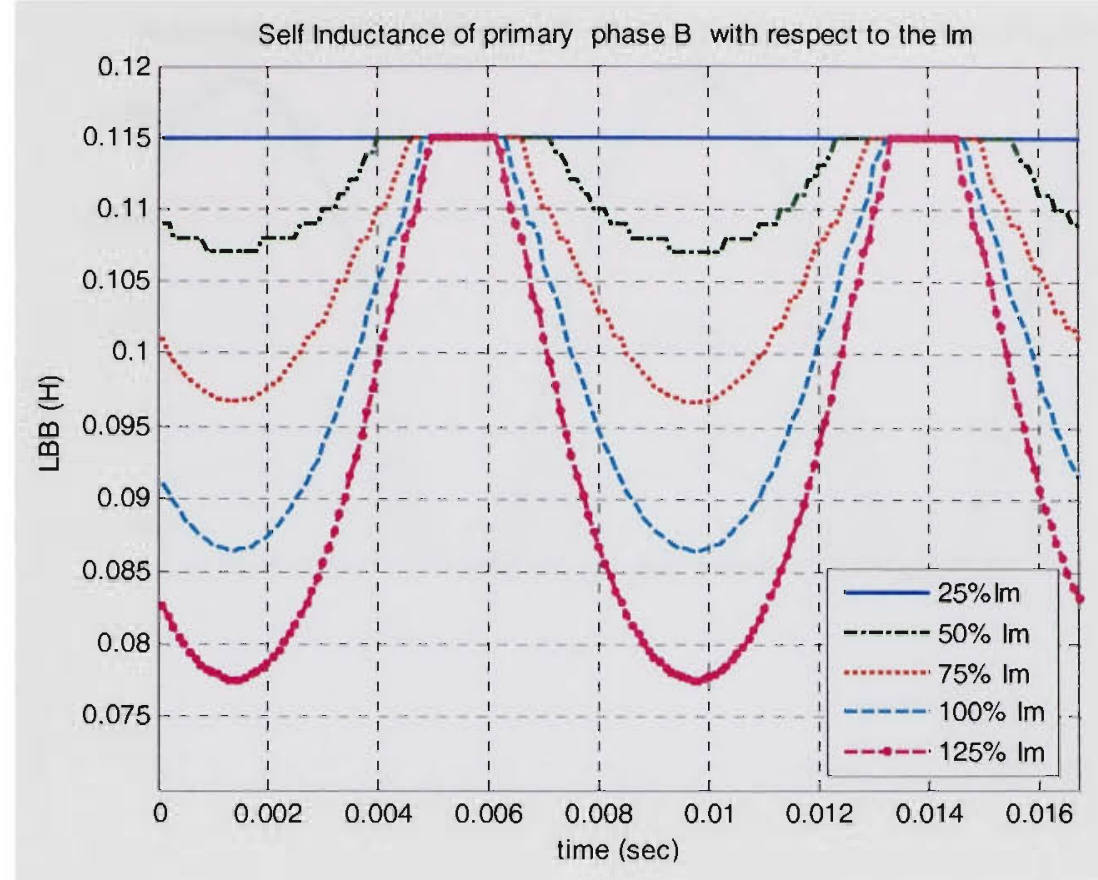

Fig.3.18. Transformer phase b primary inductance as a function of the magnetizing current

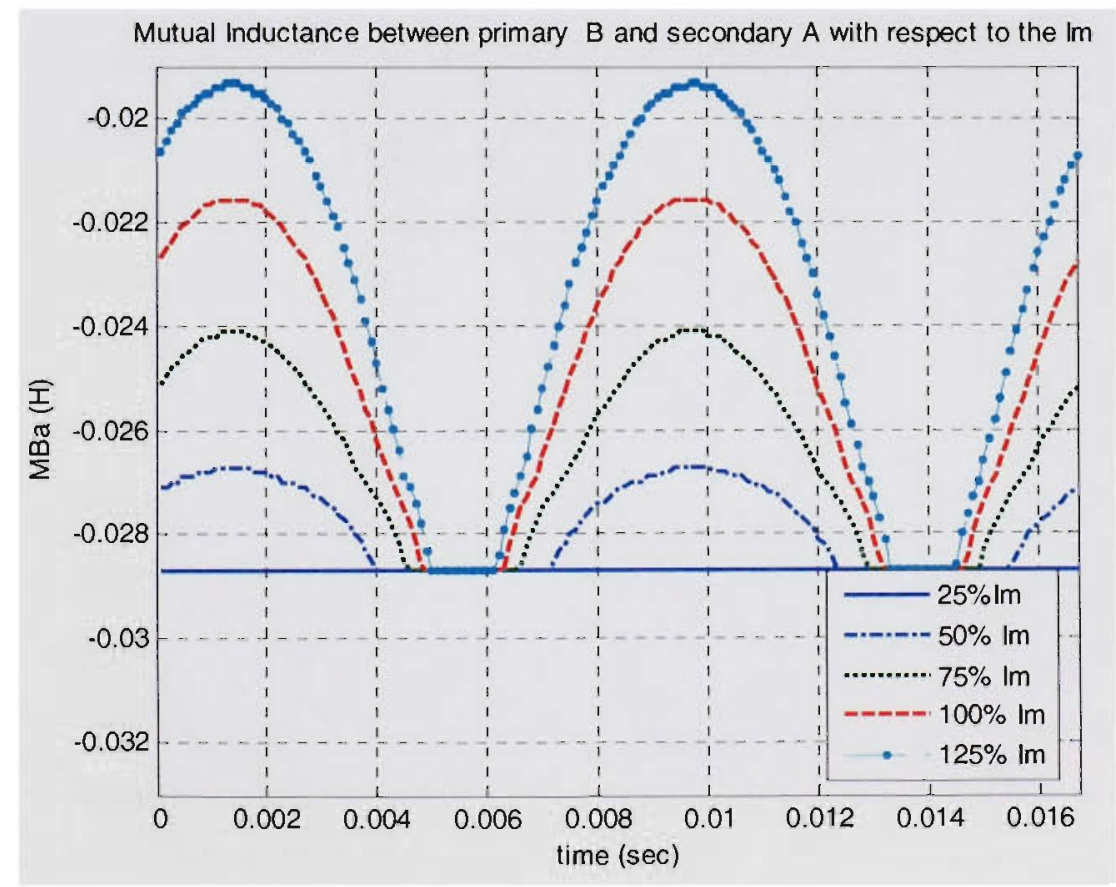

Fig.3.19. Transformer mutual inductance between phase $b$ primary and phase $c$ primary side as a function of the magnetizing current 


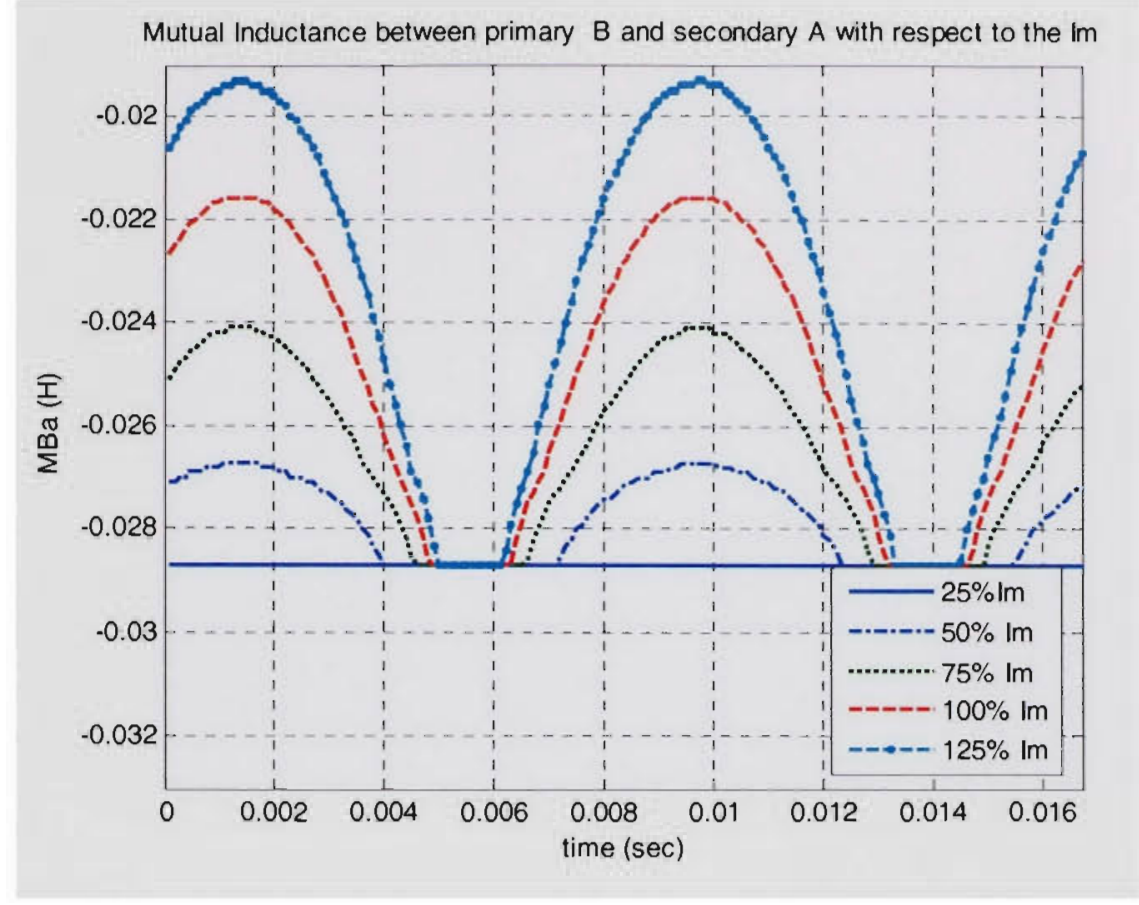

Fig.3.20. Transformer mutual inductance between phase $b$ primary and phase a secondary side as a function of the magnetizing current

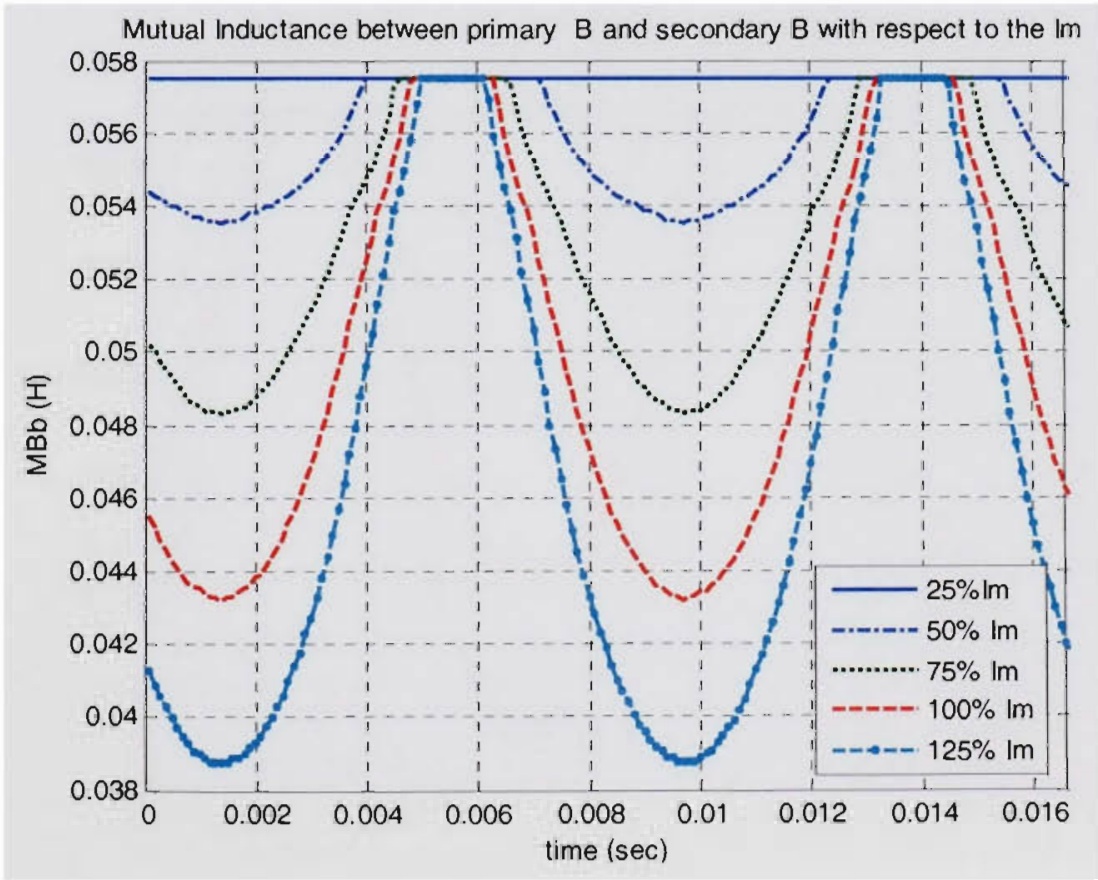

Fig.3.21. Transformer mutual inductance between phase $\mathrm{b}$ primary and phase $\mathrm{b}$ secondary side as a function of the magnetizing current 


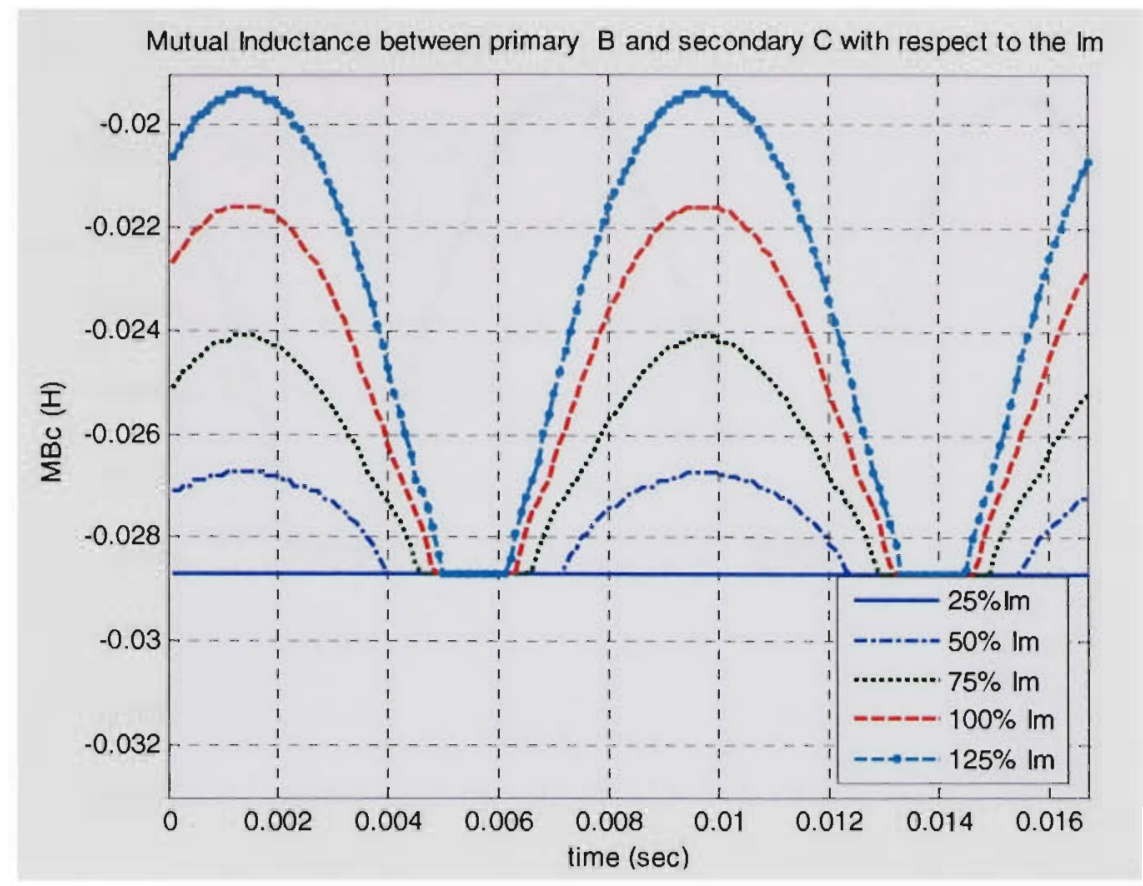

Fig.3.22. Transformer mutual inductance between phase $b$ primary and phase $c$ secondary side as a function of the magnetizing current

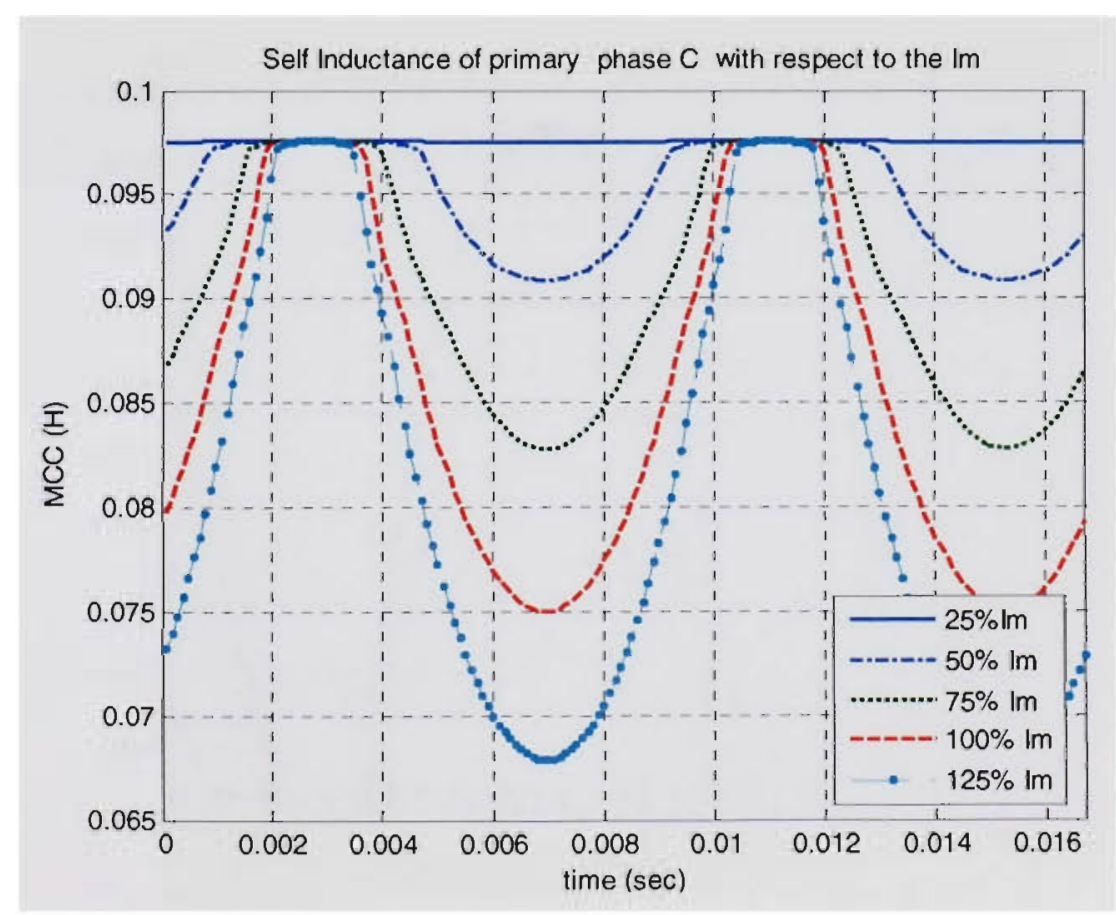

Fig.3.23. Transformer phase $\mathrm{c}$ primary inductance as a function of the magnetizing current 


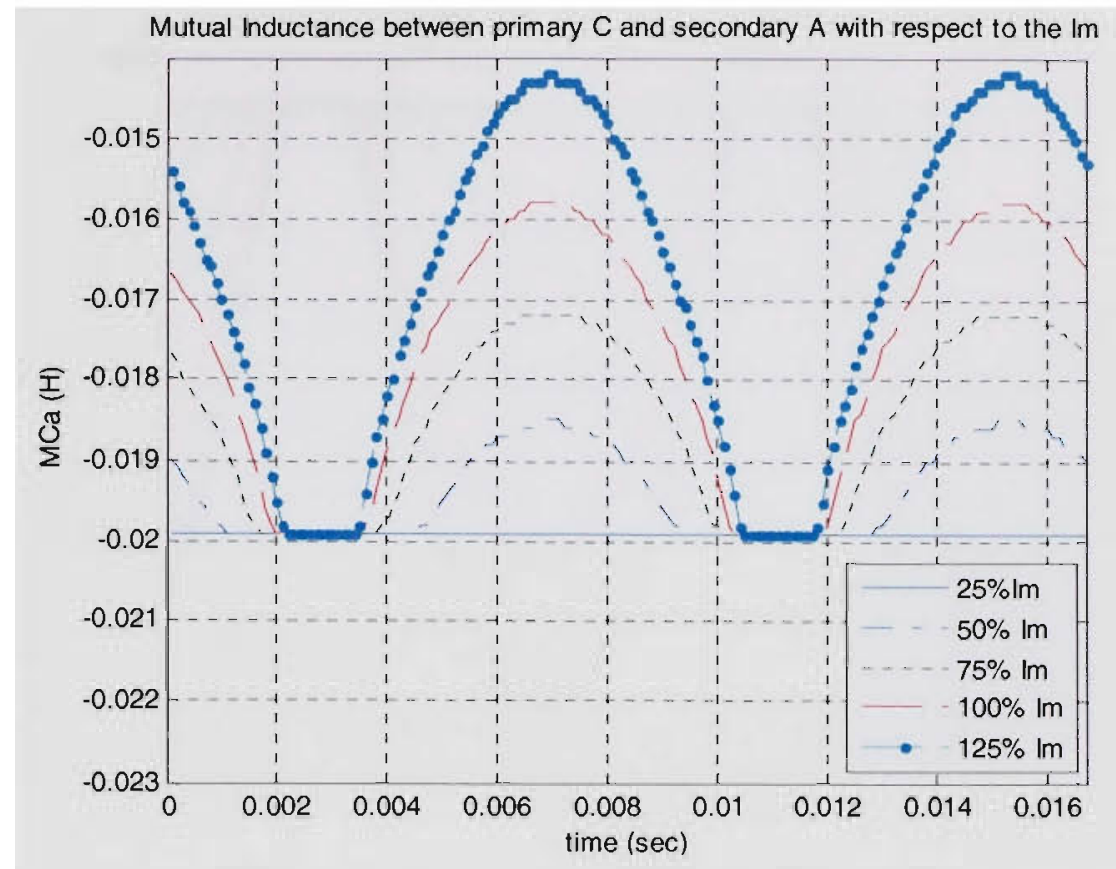

Fig.3.24. Transformer mutual inductance between phase $\mathrm{c}$ primary and phase a secondary side as a function of the magnetizing current

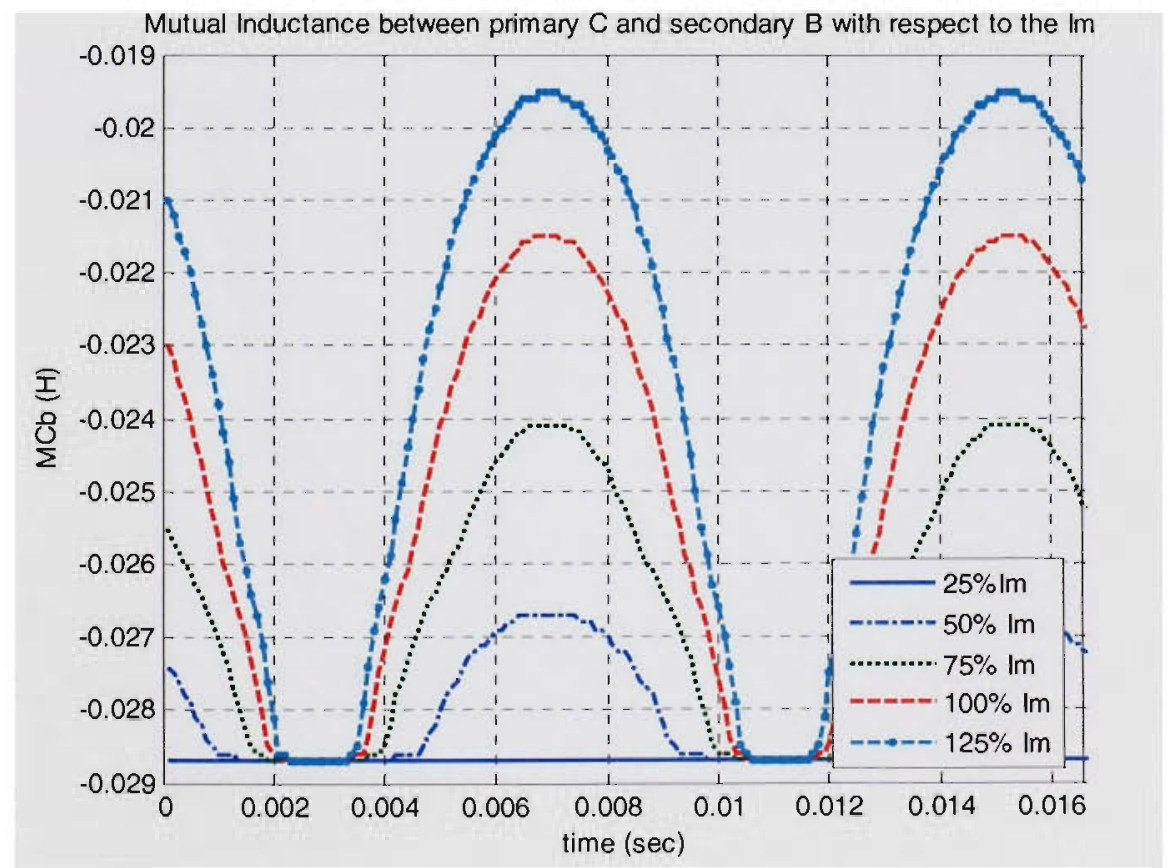

Fig.3.25. Transformer mutual Inductance between phase $\mathrm{c}$ primary and phase b secondary side as a function of the magnetizing current 


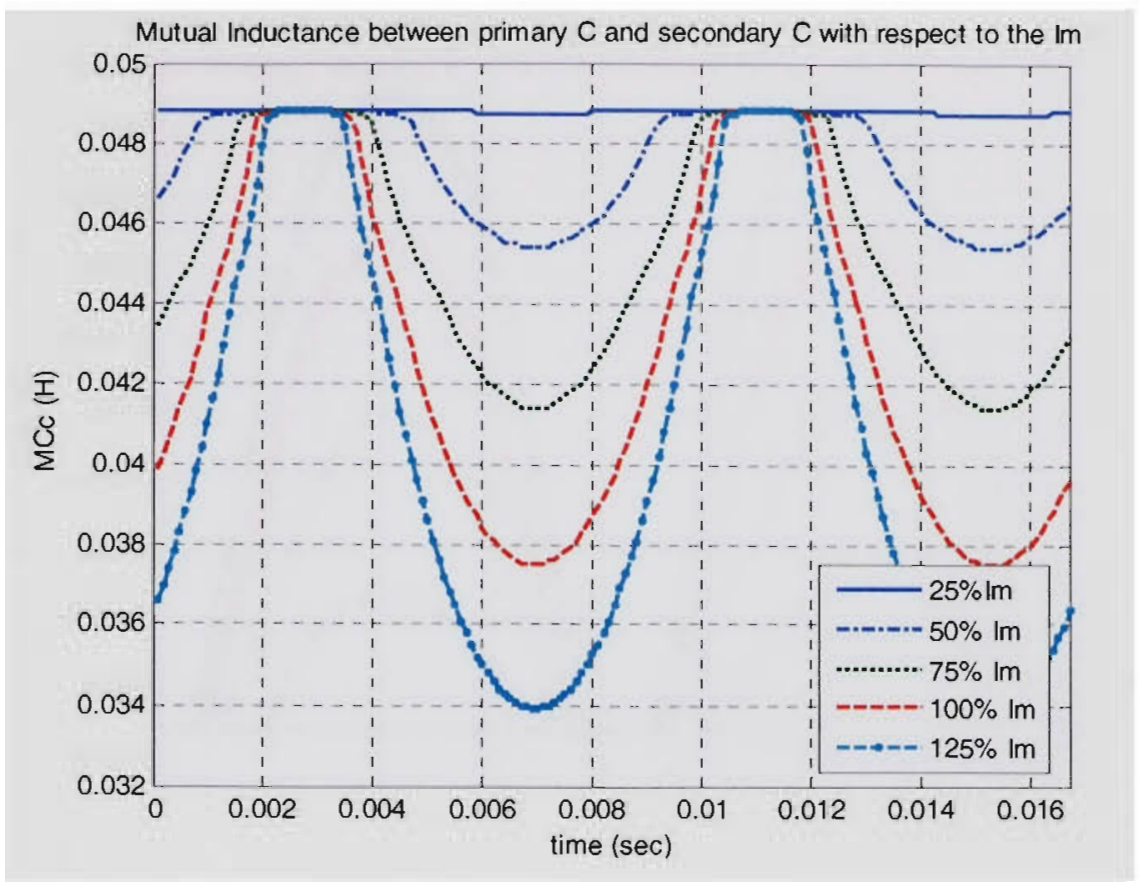

Fig.3.26. Transformer mutual inductance between phase c primary and phase c secondary side as a function of the magnetizing current

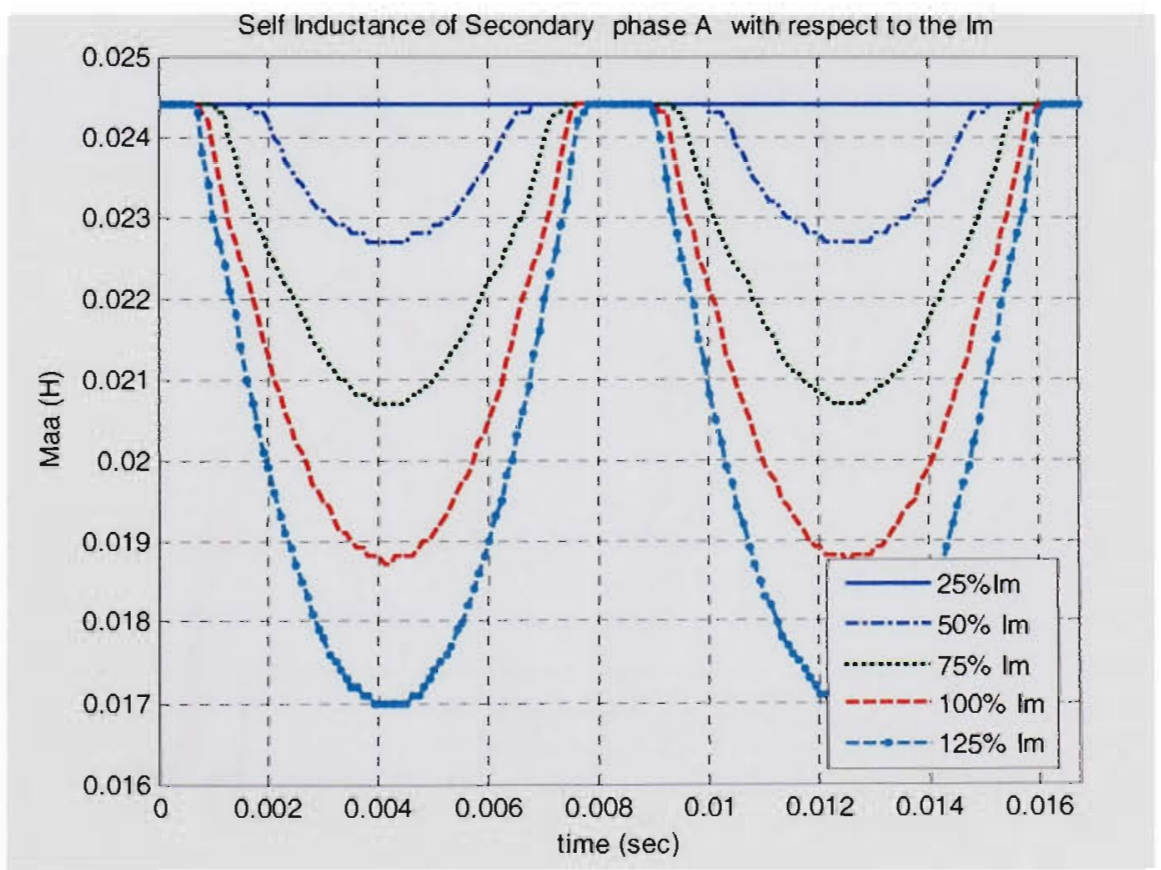

Fig.3.27. Transformer phase a secondary inductance as a function of the magnetizing current 


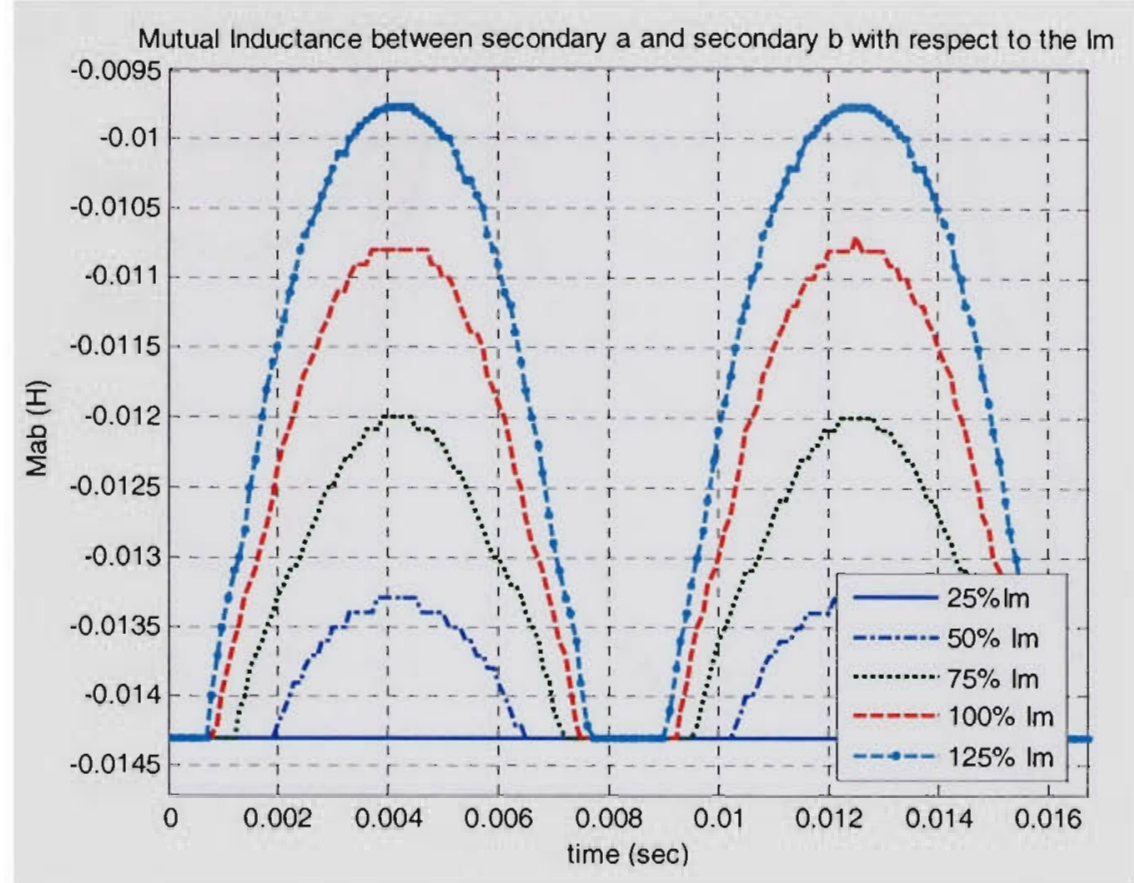

Fig.3.28. Transformer mutual inductance between phase a secondary and phase $b$ secondary side as a function of the magnetizing current

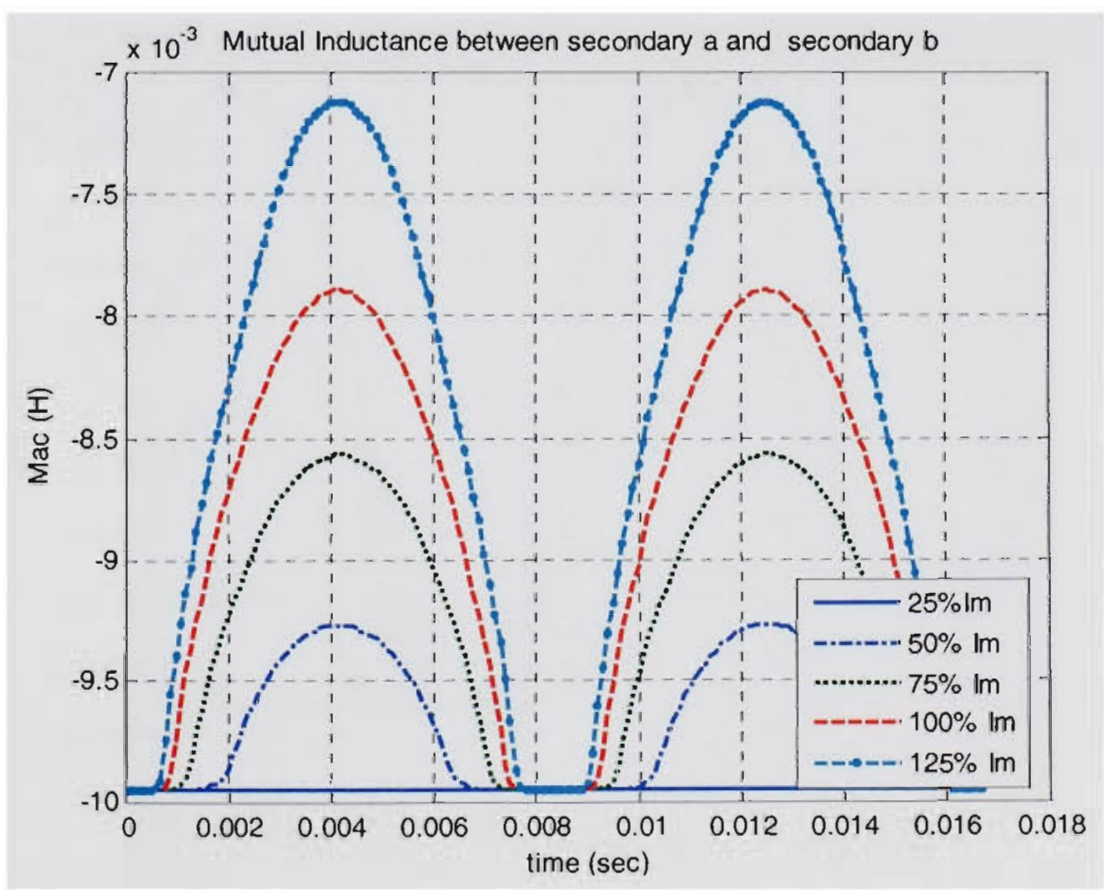

Fig.3.29. Transformer mutual inductance between phase a secondary and phase c secondary side as a function of the magnetizing current 


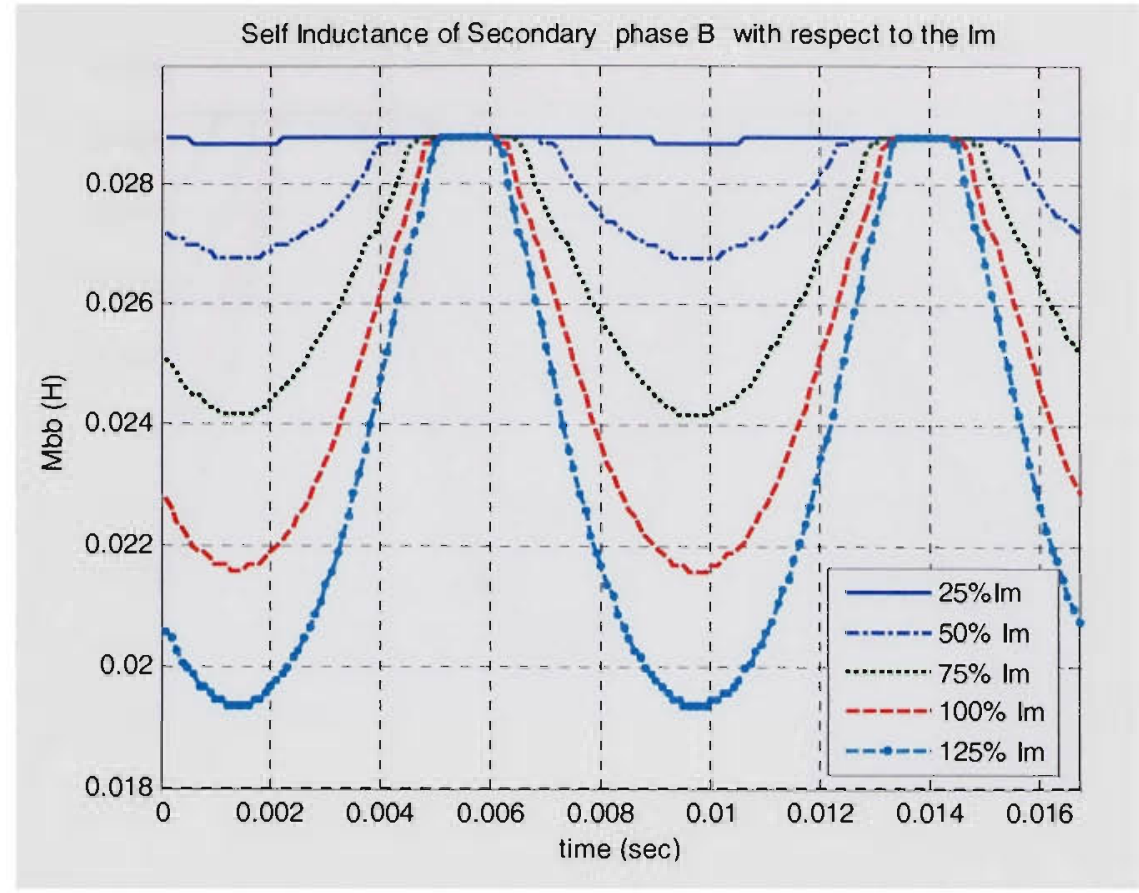

Fig.3.30. Transformer phase b secondary inductance as a function of the magnetizing current

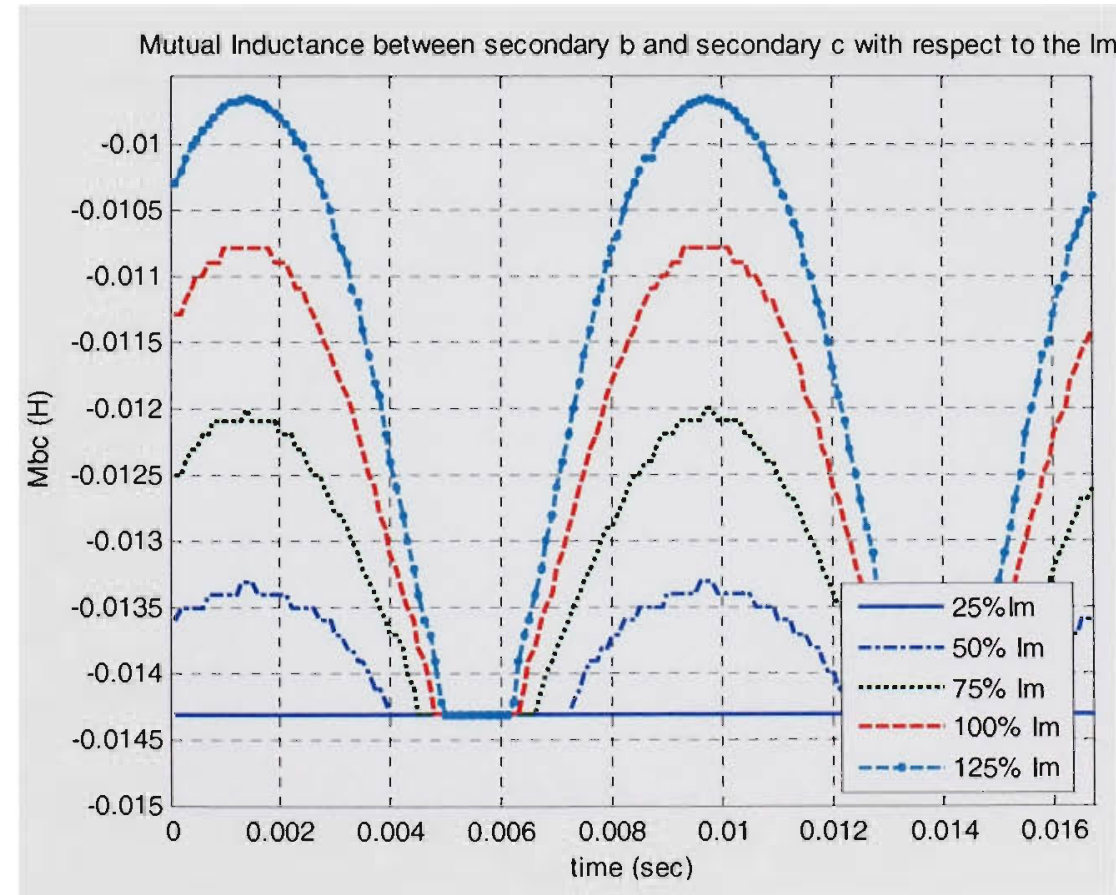

Fig.3.31. Transformer mutual inductance between phase $\mathrm{b}$ secondary and phase $\mathrm{c}$ secondary side as a function of the magnetizing current 


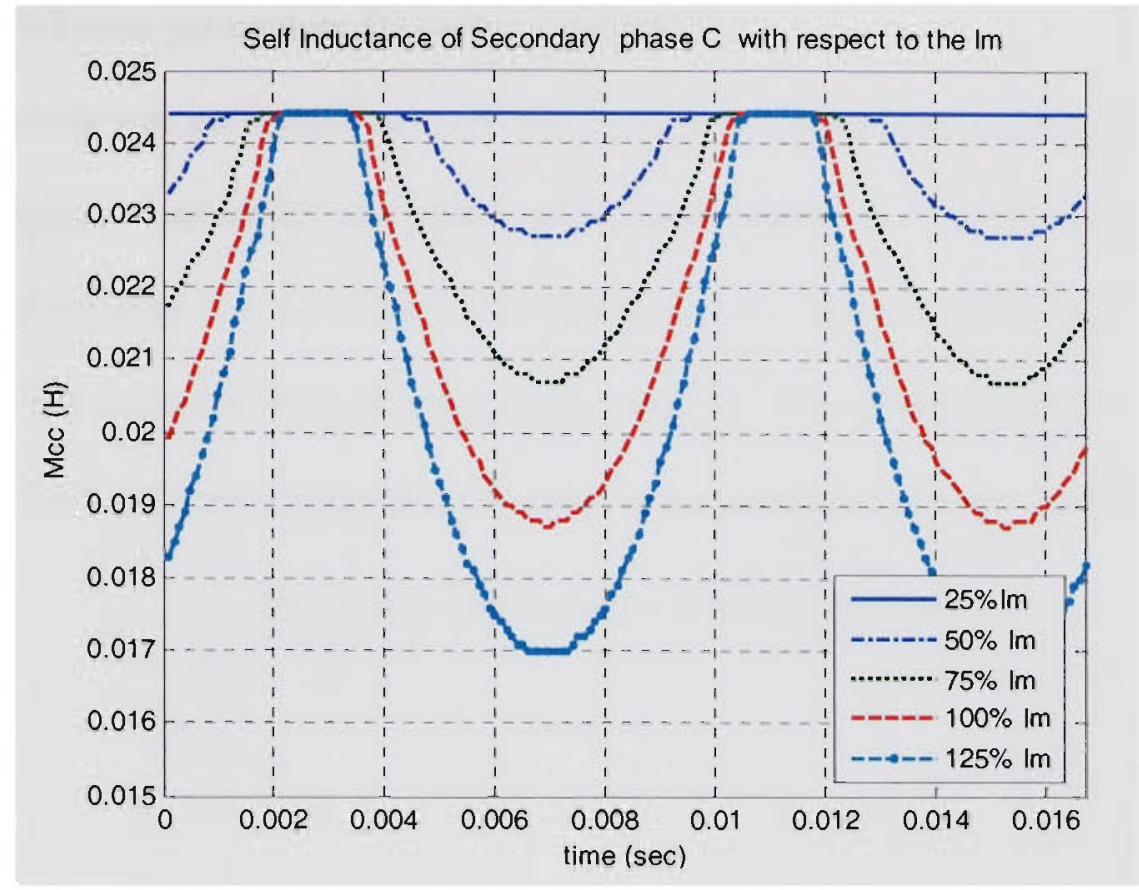

Fig.3.32. Transformer phase $\mathrm{c}$ secondary inductance as a function of the magnetizing current

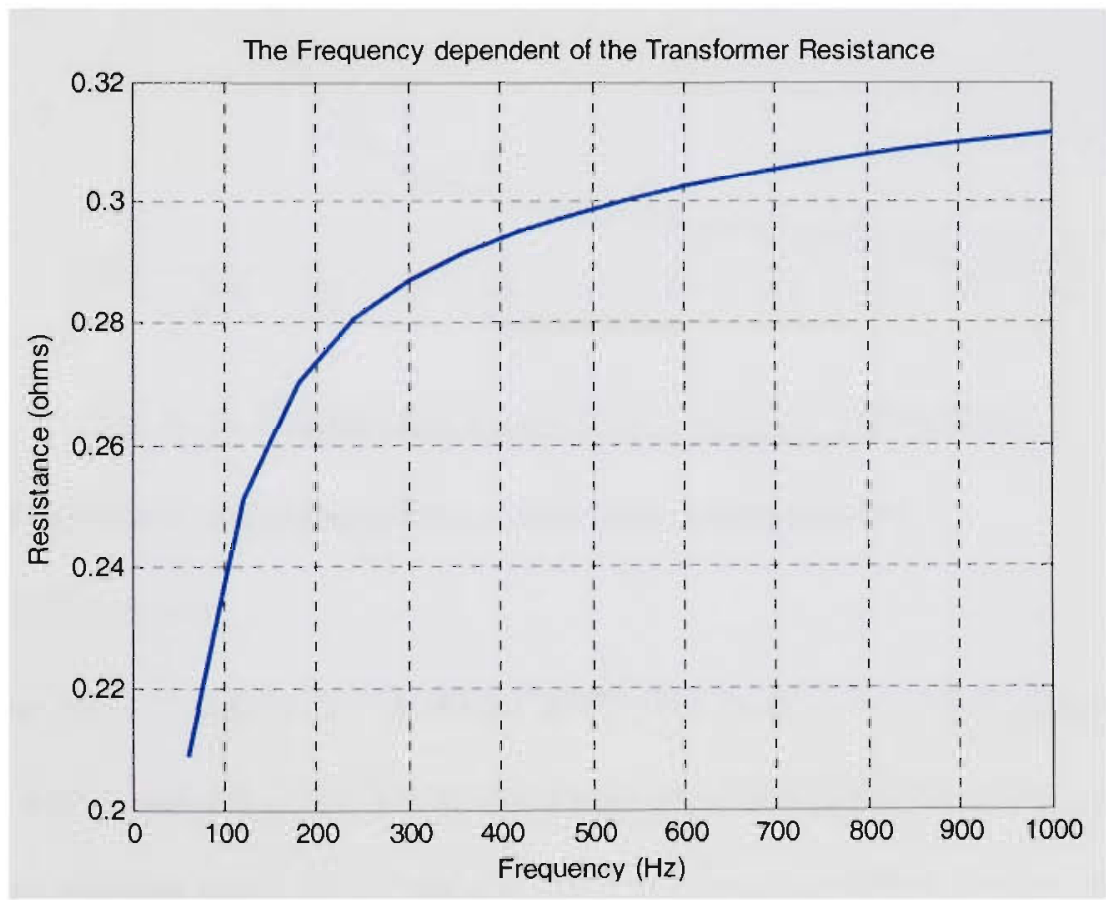

Fig.3.33. Transformer resistance frequency dependence 


\subsubsection{Transformer parameters frequency dependence}

An FE study was performed to study the variation of transformer parameters with the frequency range of our study. The results are shown in figure 3-33 and 3.34 respectively. The winding resistance is increasing with the frequency increase while the transformer inductance is decreasing with the frequency increase. The resistance has a maximum change of $49.6 \%$ increase, while the inductance has a maximum change $76.72 \%$ decrease.

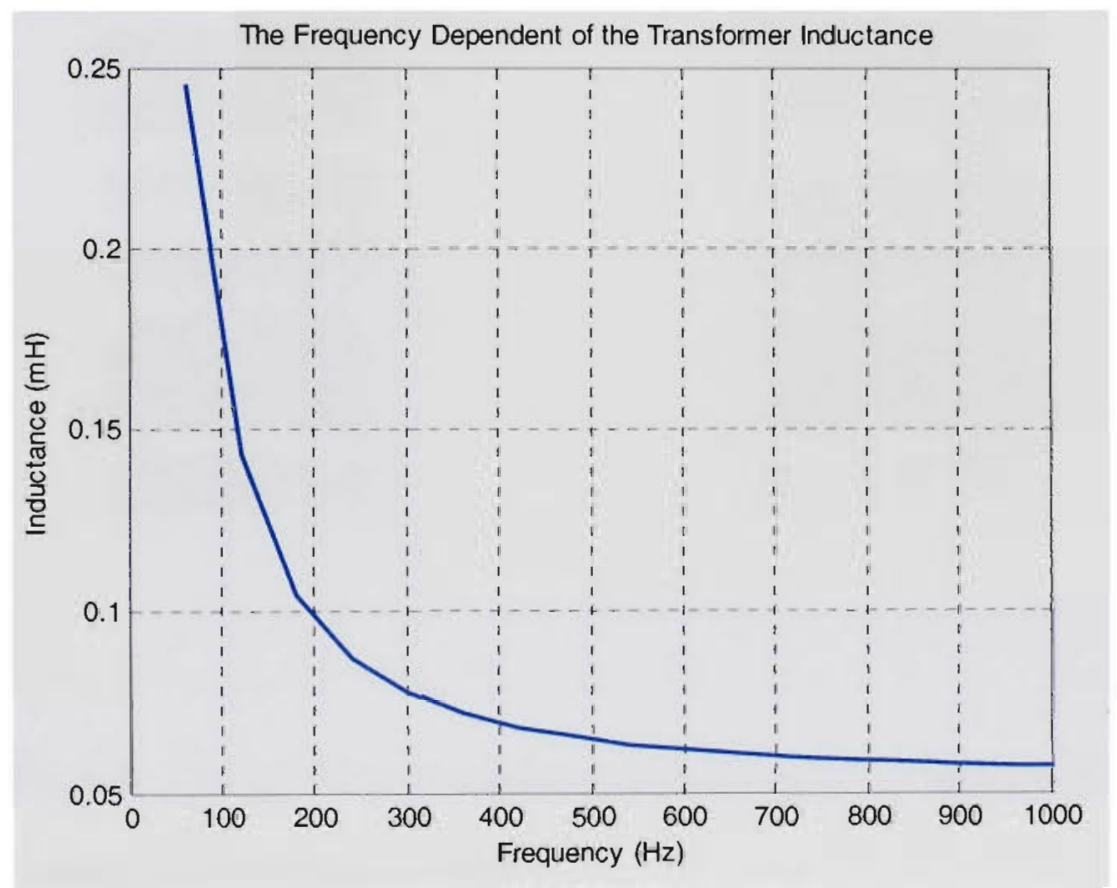

Fig.3.34. Transformer inductance frequency dependence

\subsection{Finite element modeling of the single phase transformer}

\subsubsection{The FEM model}

FEM was used to model a $125-\mathrm{kVA}, 2000 / 440 \mathrm{~V}, 60 \mathrm{~Hz}$, single phase, shell type transformer with sandwich coils. The transformer geometry is shown in Fig. 3.35.

The finite element mesh of the transformer consisting of 10477 nodes forming 5026 second order elements as illustrated in Fig. 3.35. The low and high voltage windings are 
divided into four sections and arranged in medium reactance configuration shown in Fig. 3.35. The primary and the secondary coils are represented by rectangles of corresponding materials in the FE domain.

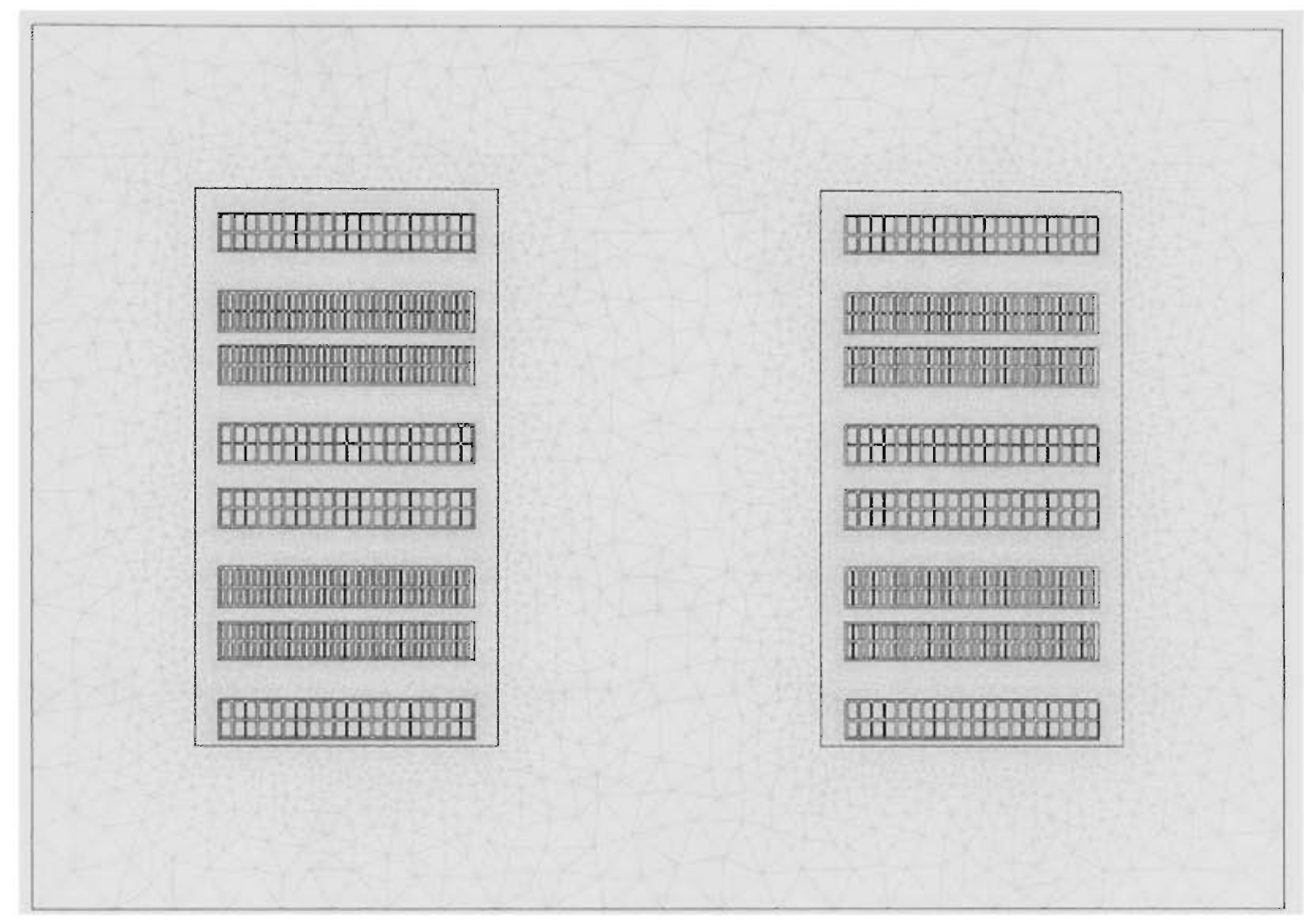

Fig.3.35. The $125 \mathrm{kVA}$ transformer geometry and the mesh details

\subsubsection{The transformer inductances profile}

The Transformer inductances were calculated using energy perturbation method $(\mathrm{E} / \mathrm{C})$ techniques described in section 2.8.1 in chapter 2. As in the previous section in order to evaluate the effect of the saturation the self and mutual inductances of the transformer, were calculated at $25 \%, 50 \%, 75 \%, 100 \%$, and $125 \%$ of the rated transformer magnetizing current. Figure 3.36 through Fig. 3.38 shows the transformer primary inductances, secondary inductances and the mutual inductance between the 
primary and secondary side as a function of the magnetizing current over a complete cycle.

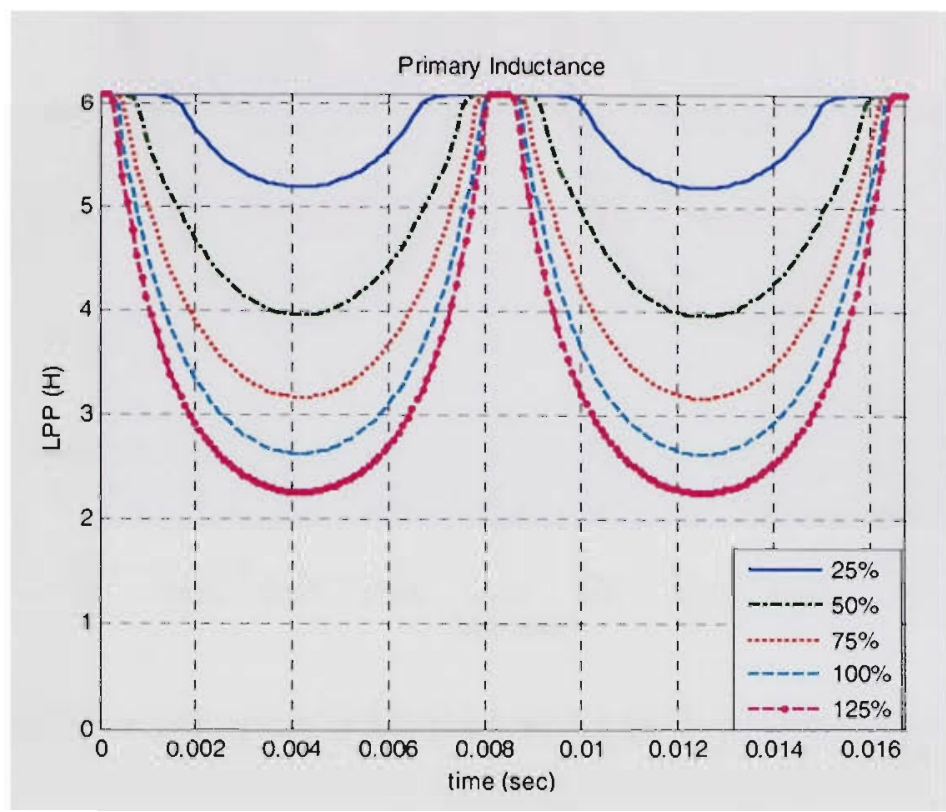

Fig.3.36. Transformer primary inductance as a function of the magnetizing current

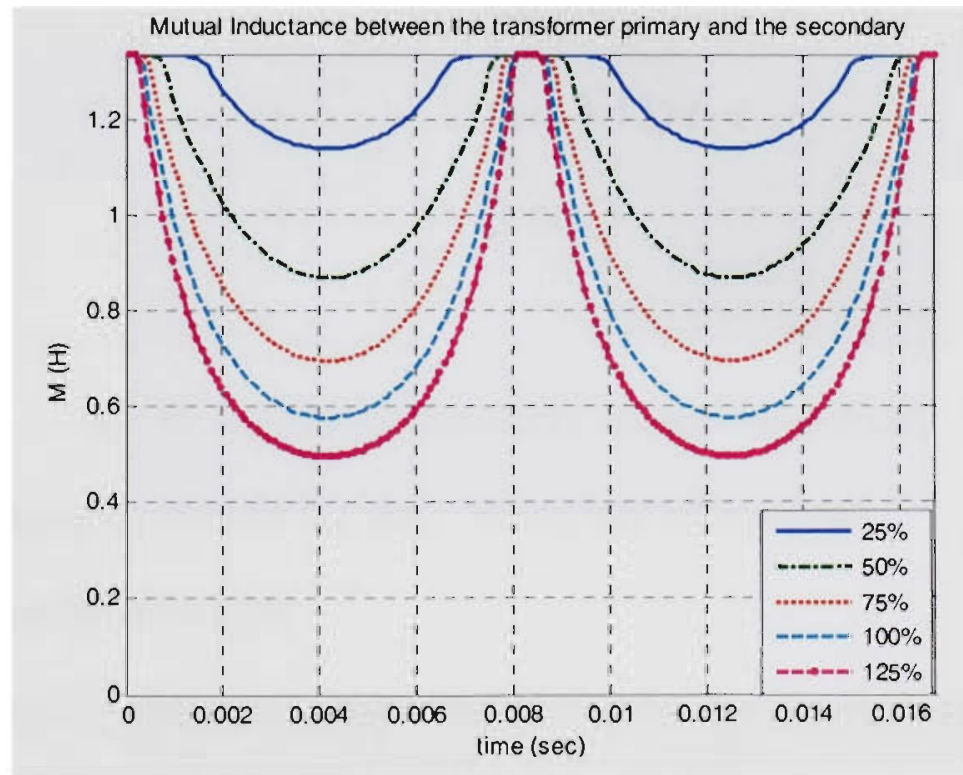

Fig.3.37. Transformer mutual inductance between the primary and secondary side as a function of the magnetizing current 


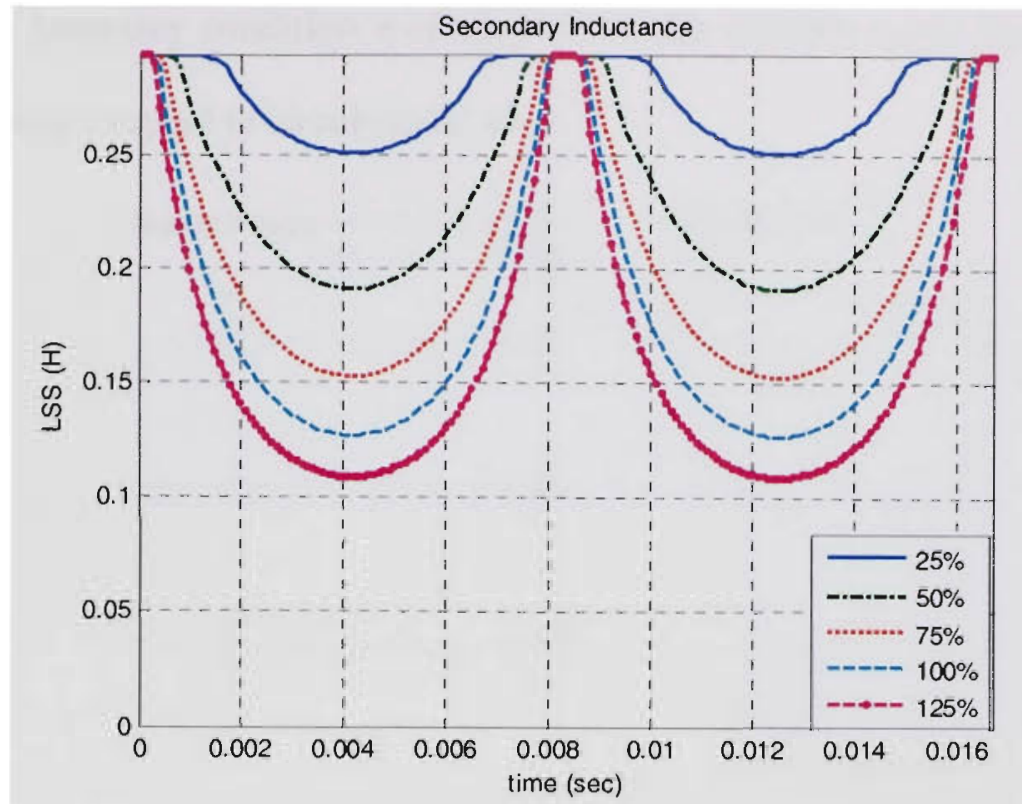

Fig.3.38. Transformer secondary inductance as a function of the magnetizing current

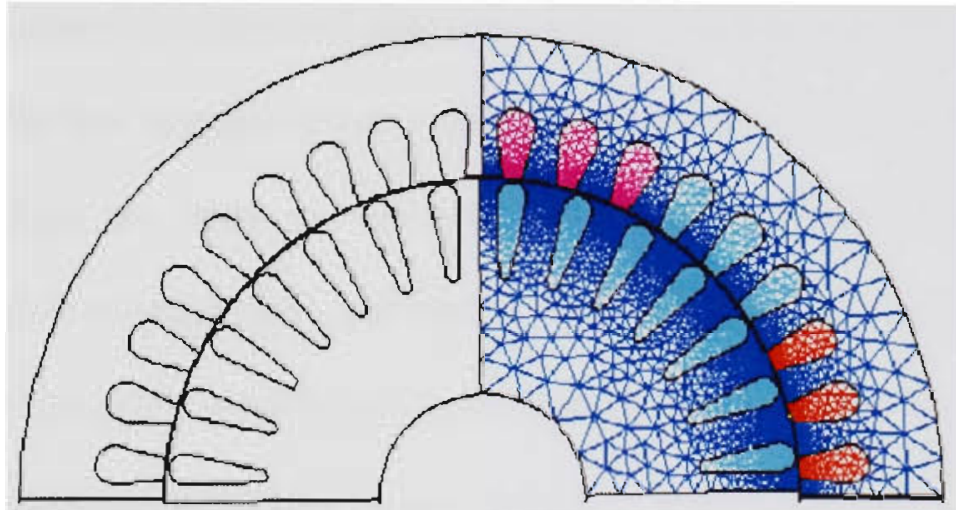

Fig.3.39. Induction Motor finite element mesh detail

\subsection{Finite element model of the three phase Induction motor}

\subsubsection{The FE model description}

FEM was used to model a 3-phase, $380 \mathrm{~V}, 4$ pole, Y-connected, 36 stator slots, 28 rotor bars, $1751 \mathrm{rpm}, 60 \mathrm{~Hz}$, induction motor. The conductors have constant conductivities. The winding is double layered. The stator and rotor iron cores are isotropic non-linear magnetic material. 
Dirichlet boundary condition was adopted on the machine outer diameter. The FE analysis has been coupled to circuit simulation.

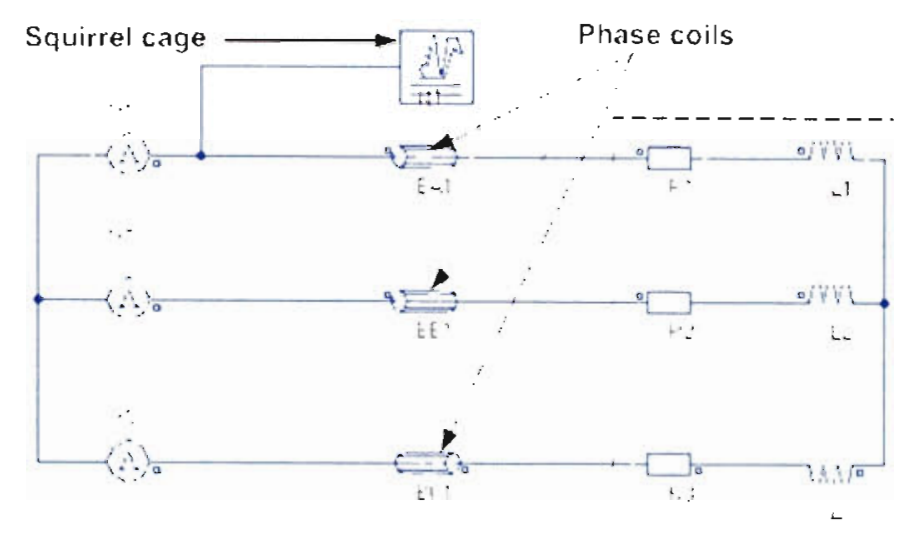

Bar end impedances

Fig.3.40. Circuit domain model of the induction motor

The phase currents in stator and rotors are solved together with the magnetic vector potential using the line voltages as input variables as described in chapter 2 . The external circuit connections are used to describe the electrical connectivity between the conducting regions, external loads, and power supplies. The rotor cage end rings and bar ends impedances are represented in the circuit domain. Due to the symmetry, only quarter of the motor is modeled. The finite element mesh of the motor covers one quarter of the cross section, as illustrated in Fig. 3.39.

The finite element mesh of the motor consists of 43721 nodes forming 21822 second order elements. The mesh size is selected in coordination with the supply frequency.

Figure 3.40 shows the circuit domain model of the whole system with induction coils, the voltage source, and the rotor cage end rings and bar ends impedances. The voltage supply of the motor is sinusoidal and symmetric in both stator and rotor. Fig. 3.41 shows the motor three phase starting currents obtained from the FE model. 


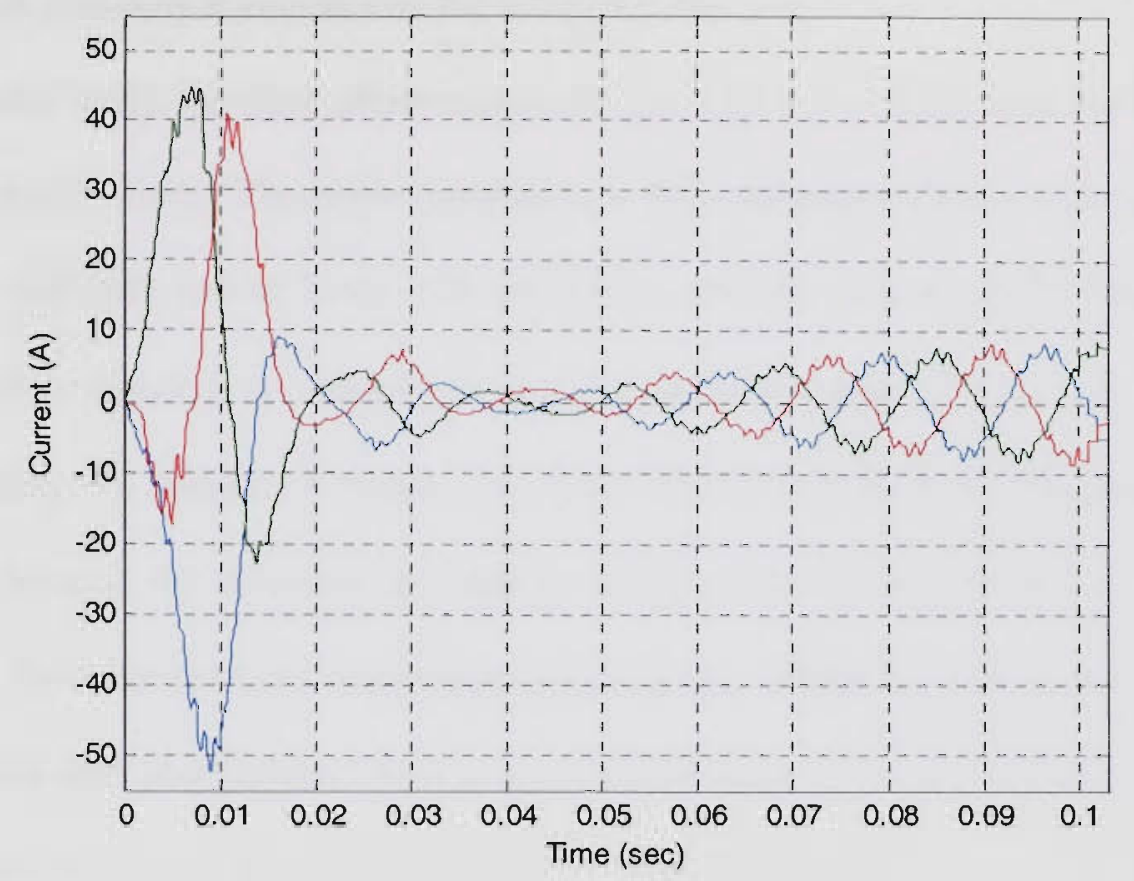

Fig.3.41. Induction motor three phase starting currents
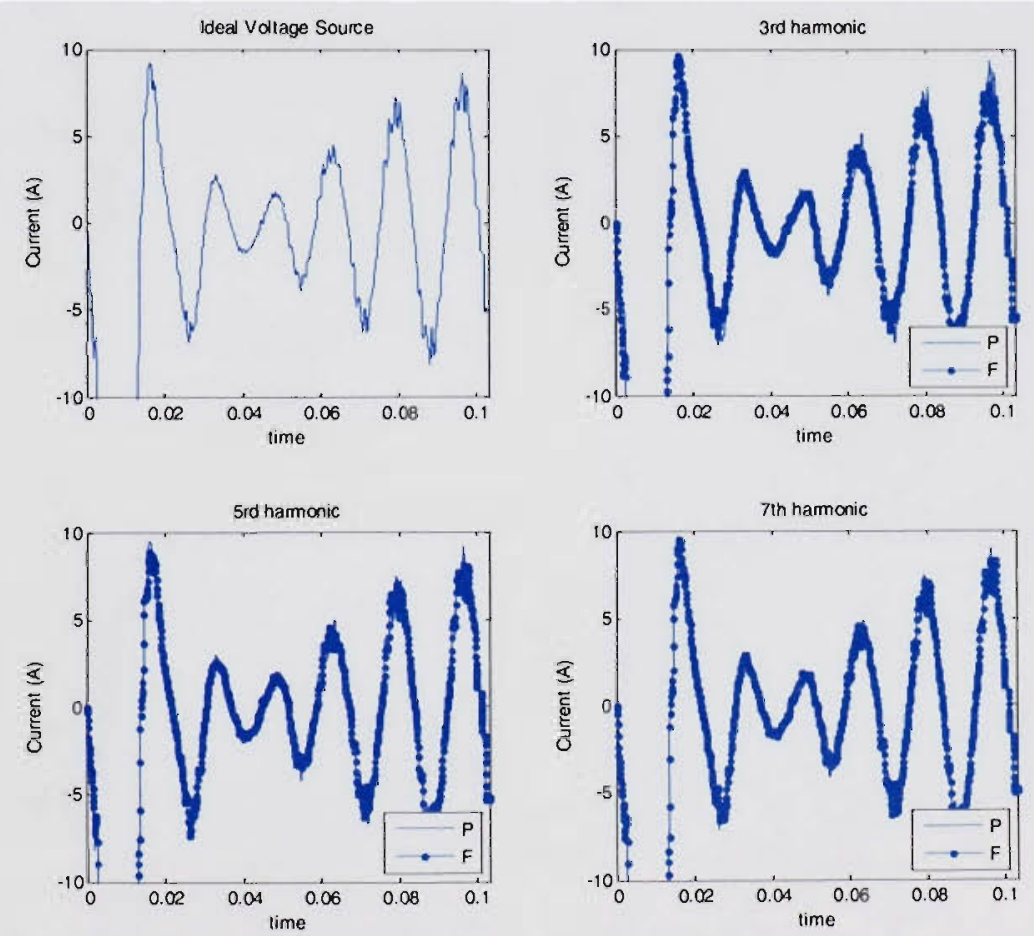

Fig.3.42. Induction motor phase a current for ideal supply and supply distorted by the $3^{\text {rd }}$, $5^{\text {th }}$, and $7^{\text {th }}$ harmonics 


\subsubsection{Effect of supply harmonics on the induction machine}

In this case study, the three-phase cage induction motor is connected to the harmonic distorted voltage source. The motor resistance is frequency-dependent due to conductor skin effects and eddy current losses. The resistance normally increases with frequency in the form of $h^{\alpha}$, where $\mathrm{h}$ is the harmonic order and $\alpha$ is in the range of 0.5 to 1.5 .

We conducted a number of studies with wide variation of harmonic characteristics in the supply voltage. We distorted the supply voltage with odd harmonics up to the $7^{\text {th }}$ component. For each harmonic component added to the voltage waveform, there are two cases; peaking case and flattened peak case. In the peaking case, the positive peak of the harmonic waveform coincides with the positive fundamental peak.

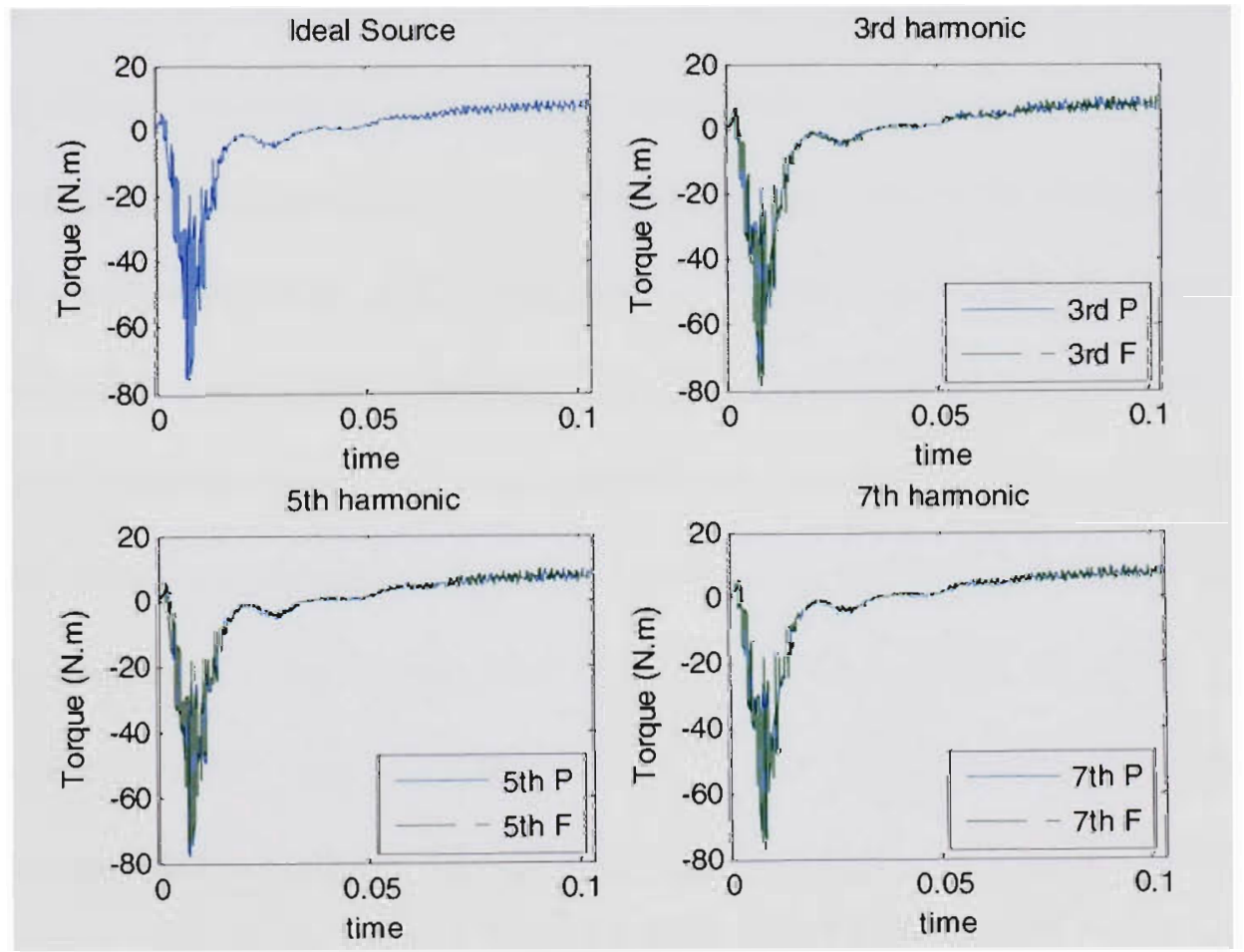

Fig.3.43. Induction motor torque profile for ideal supply and supply distorted by $3^{\text {rd }}, 5^{\text {th }}$, and $7^{\text {th }}$ harmonics 
In the flattened peak case, the negative peak of the harmonic component coincides with the positive fundamental peak.

Figure 3-42 and 3-43 shows the induction motor three phase stator currents and the toque waveforms obtained from FEM under different ideal, $3^{\text {rd }}$ harmonic distorted, $5^{\text {th }}$ harmonic distorted, and $7^{\text {th }}$ harmonics distorted voltage source respectively.

The torque ripples increase in the presence of harmonics. The peaked harmonic case is the worst case of torque ripple for all the harmonics.

\subsection{Finite element modeling of three plıase cable}

\subsubsection{The used geometry}

Cables are important element in the shipboard power system transient analysis. Analytical Formulas can not be used to calculate the cable parameters because of the proximity effect. Anaytical formulas are not suitable for complex geometries [203]. Two cable geometries were modeled using FEM, three phase cable with triangle conductors and three phase cable with circle conductors. The first cable type consists of three triangle sectors phase copper conductors. The cable insulation system consists of inner insulators between the phase conductors and the protective braiding and outer insulators around the protective braiding. The phase conductor's sharp corners of are beveled to reduce the field stress at the corners. The cable geometry is shown in Fig 3.44.

The second cable type consists of three circle sectors phase conductors. All conductors are made of copper. The cable insulation system consists of inner and outer insulators and a protective braiding (steel tape). The finite element mesh of the cable consists of 50157 nodes forming 25030 second order elements. The cable geometry and the mesh details are shown in Fig 3.45. 
In classical two-dimensional modeling of cables, several idealizing assumptions are usually made:

1. The cable is assumed to be long and thus the FE model becomes a twodimensional one.

2. The conductors have constant conductivities and relative permeabilities.

3. The steel tape has a constant conductivity but its relative permeability is nonlinear permeability defined with a model of analytic saturation curve with bend adjustment.

Figure 3-46 and 3-47 shows the Magnetic Flux Density distribution inside the triangle and circle cable respectively at $\mathrm{t}=10 \mathrm{msec}$. The finite element mesh of the first type cable consists of 69005 nodes forming 34424 second order elements.

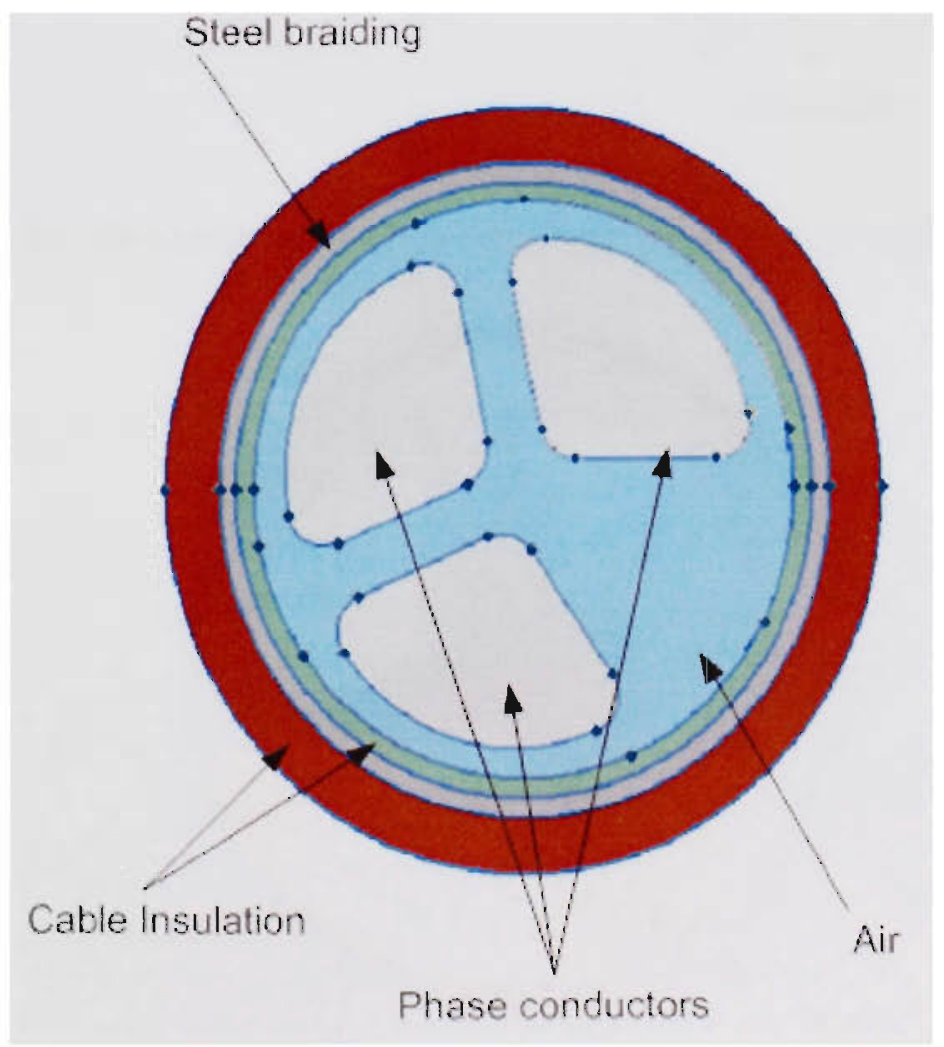

Fig.3.44. The triangle conductors cable geometry 
Dirichlet boundary condition was adopted on the external circle of infinite region surrounding the cable.

\subsubsection{Low frequency parameters calculation}

The triangle conductors cable inductances were calculated by energy perturbation method described in chapter 2. The inductances matrix results are shown in Table 3-1.

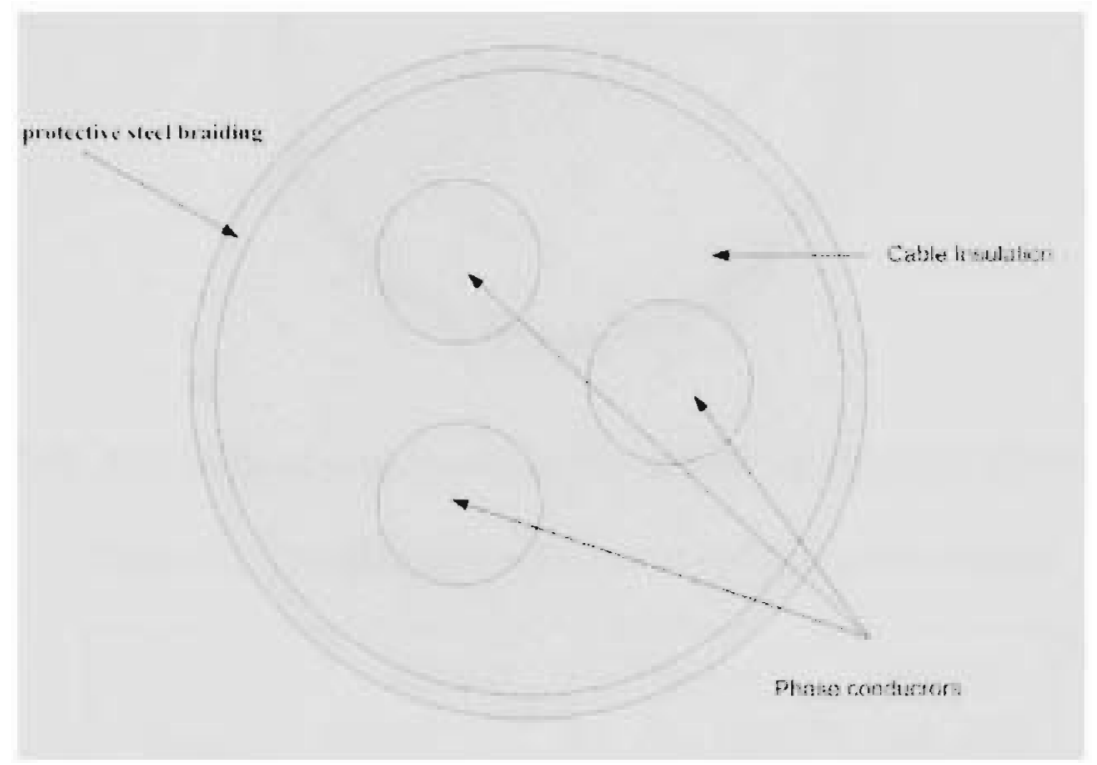

Fig. 3.45. The circle conductors cable type geometry and mesh details

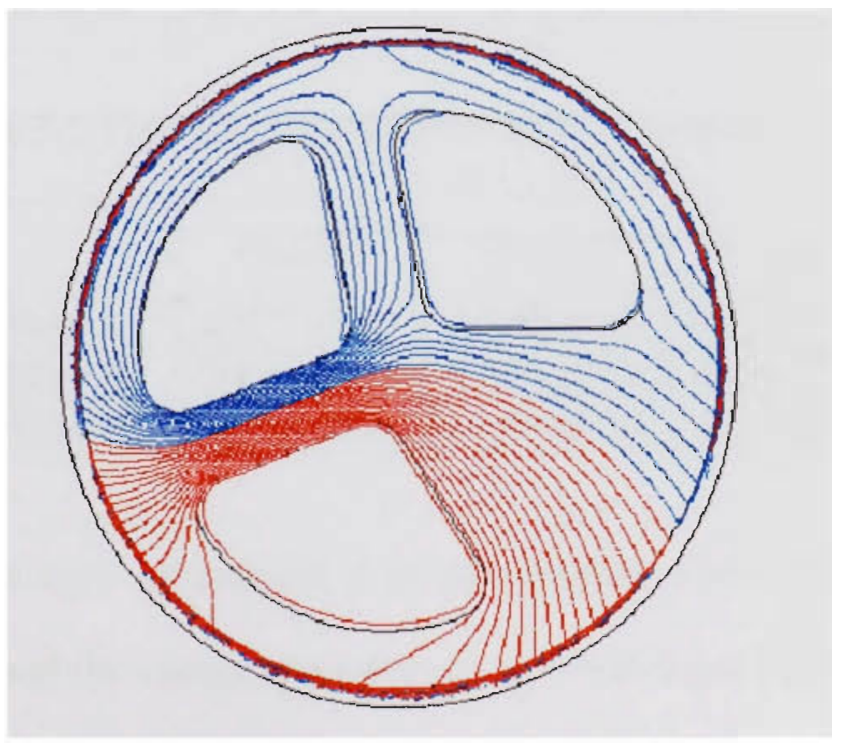

Fig.3.46. The triangle conductors cable flux density distribution@ @ $\mathrm{t}=10 \mathrm{msec}$ 
The capacitance matrix was then calculated using the energy approach described in section 2.8.3. The results are shown in Table 3-2.

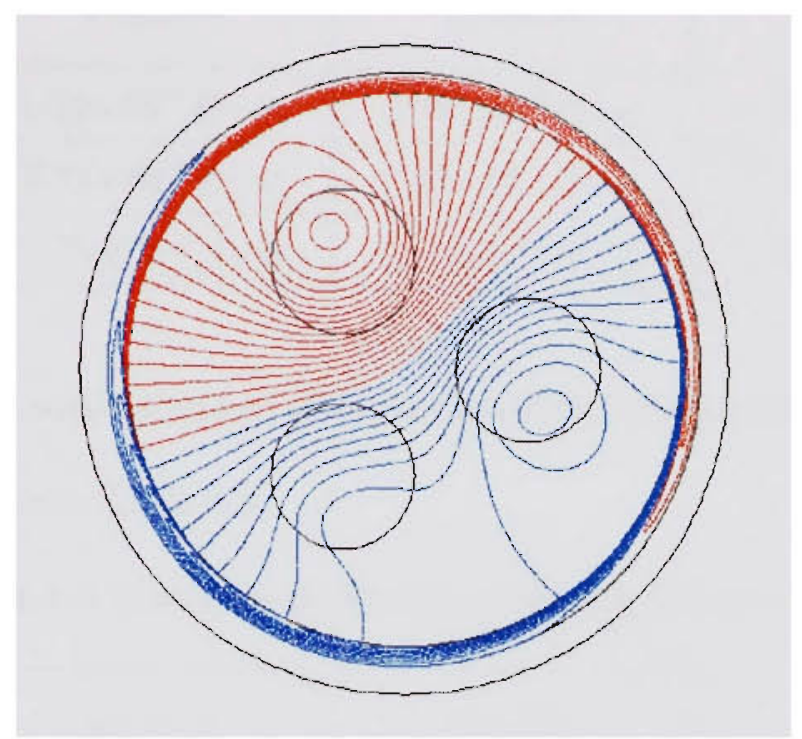

Fig.3.47. The circle conductors cable Flux density distribution@ $@ \mathrm{t}=10 \mathrm{msec}$

Table 3-1 The triangle conductors cable inductance matrix

\begin{tabular}{|c|c|c|c|}
\hline & Phase A & Phase B & Phase C \\
\hline Phase A & $0.113 \mu \mathrm{H} / \mathrm{m}$ & $0.112 \mu \mathrm{H} / \mathrm{m}$ & $0.111 \mu \mathrm{H} / \mathrm{m}$ \\
\hline Phase B & $0.112 \mu \mathrm{H} / \mathrm{m}$ & $0.113 \mu \mathrm{H} / \mathrm{m}$ & $0.112 \mu \mathrm{H} / \mathrm{m}$ \\
\hline Phase C & $0.111 \mu \mathrm{H} / \mathrm{m}$ & $0.112 \mu \mathrm{H} / \mathrm{m}$ & $0.113 \mu \mathrm{H} / \mathrm{m}$ \\
\hline
\end{tabular}

Table 3-2 The triangle conductors cable capacitance matrix

\begin{tabular}{|c|c|c|c|}
\hline & Phase A & Phase B & Phase C \\
\hline Phase A & $0.277 \mathrm{pF} / \mathrm{m}$ & $0.066 \mathrm{pF} / \mathrm{m}$ & $0.023 \mathrm{pF} / \mathrm{m}$ \\
\hline Phase B & $0.066 \mathrm{pF} / \mathrm{m}$ & $0.306 \mathrm{pF} / \mathrm{m}$ & $0.066 \mathrm{pF} / \mathrm{m}$ \\
\hline Phase C & $0.023 \mathrm{pF} / \mathrm{m}$ & $0.066 \mathrm{pF} / \mathrm{m}$ & $0.277 \mathrm{pF} / \mathrm{m}$ \\
\hline
\end{tabular}

The second cable type inductances and capacitances were calculated by energy perturbation method and the energy approach as described above. The results are shown in Table 3-3 and 3-4 respectively. 
Table 3-3 The circle conductors cable inductance Matrix

\begin{tabular}{|c|c|c|c|}
\hline & Phase A & Phase B & Phase C \\
\hline Phase A & $1.89 \times 10^{-7} \mathrm{H} / \mathrm{m}$ & $2.75 \times 10^{-8} \mathrm{H} / \mathrm{m}$ & $2.75 \times 10^{-8} \mathrm{H} / \mathrm{m}$ \\
\hline Phase B & $2.75 \times 10^{-8} \mathrm{H} / \mathrm{m}$ & $1.89 \times 10^{-7} \mathrm{H} / \mathrm{m}$ & $2.75 \times 10^{-8} \mathrm{H} / \mathrm{m}$ \\
\hline Phase C & $2.75 \times 10^{-8} \mathrm{H} / \mathrm{m}$ & $2.75 \times 10^{-8} \mathrm{H} / \mathrm{m}$ & $1.89 \times 10^{-7} \mathrm{H} / \mathrm{m}$ \\
\hline
\end{tabular}

The capacitance matrix was calculated utilizing the energy approach described in Chapter 2. The results are shown in Table 3-4.

Table 3-4 The circle conductors cable capacitance matrix

\begin{tabular}{|l|c|c|c|}
\hline & Phase A & Phase B & Phase C \\
\hline Phase A & $1.934 \times 10^{-10} \mathrm{~F} / \mathrm{m}$ & $0.4269 \times 10^{-10} \mathrm{~F} / \mathrm{m}$ & $0.4269 \times 10^{-10} \mathrm{~F} / \mathrm{m}$ \\
\hline Phase B & $0.4269 \times 10^{-10} \mathrm{~F} / \mathrm{m}$ & $1.934 \times 10^{-10} \mathrm{~F} / \mathrm{m}$ & $0.4269 \times 10^{-10} \mathrm{~F} / \mathrm{m}$ \\
\hline Phase C & $0.4269 \times 10^{-10} \mathrm{~F} / \mathrm{m}$ & $0.4269 \times 10^{-10} \mathrm{~F} / \mathrm{m}$ & $1.934 \times 10^{-10} \mathrm{~F} / \mathrm{m}$ \\
\hline
\end{tabular}

\subsubsection{High frequency parameters calculation}

Under PWM operating condition the cable resistance, inductance and capacitance behave differently from the low frequency operation. The conductors have skin and proximity effects which cause resistance to be much higher than its value at low frequency. The inductance value decreases with an increase in the operating frequency while the capacitance effect comes in the picture at high frequency. The calculation of the cable frequency dependent impedance is very crucial for the shipboard power system transient analysis.

A magnetodynamic FE analysis was performed to obtain the two cable frequency response by varying the primary voltage source frequency with the other side is short 
circuited. In order to mesh the conductor region for the high frequency eddy current problem, care must be taken such that the size of the elements used should be smaller than the skin depth associated with the supply frequency. Fig. 3.48 shows the mesh details for the circle conductor cable.

Figure 3.49 and 3.50 shows respectively the current distribution change and the variation of the cable resistance and inductance with the frequency change for the triangle conductor's cable.

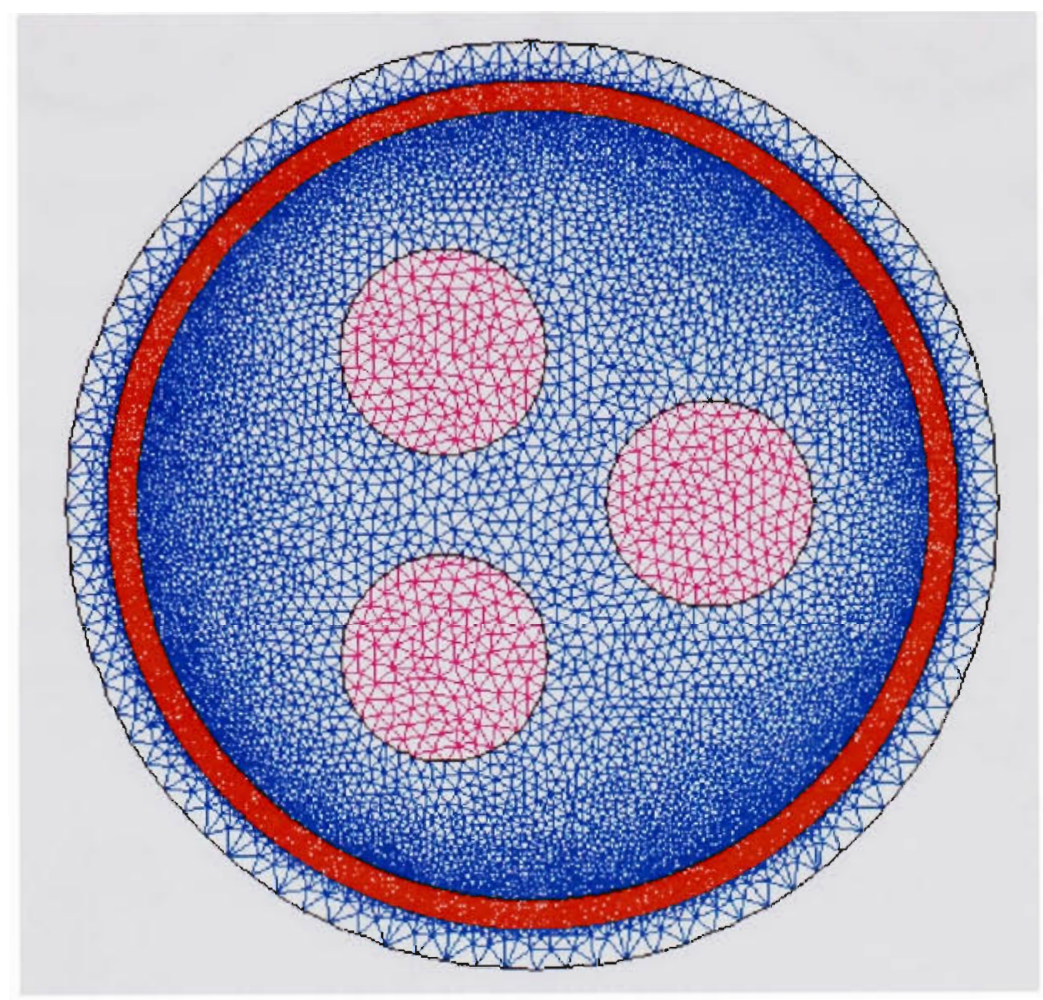

Fig.3.48. The circle conductors cable mesh detail for the high frequency study

Figure 3.51 and 3.52 shows respectively the current distribution change and the variation of the cable resistance and inductance with the supply frequency change for the circle conductor's cable. The cable resistance value increased with the frequency increase while the inductance value decreased with the frequency increase. 
The simultaneous excitation of phase conductors is performed to consider the proximity effects. The losses associated with the proximity effect are losses in a conductor due to currents in all other conductors. Proximity effects are far more than skin effects while calculating the resistance values at high frequency operation.

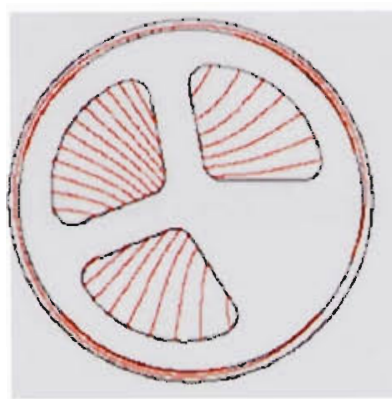

(a) $60 \mathrm{~Hz}$

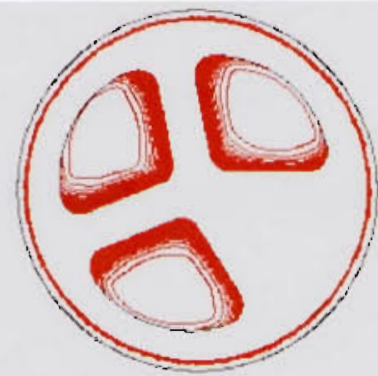

$@ 8060 \mathrm{~Hz}$

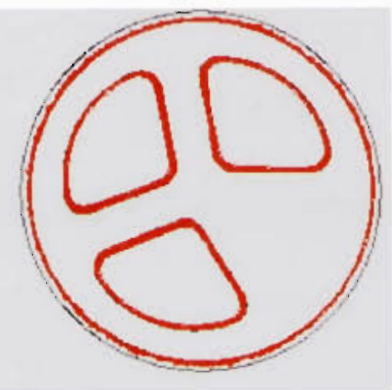

$@ 100 \mathrm{kHz}$

Fig.3.49. The triangle conductors cable current distribution at different frequencies

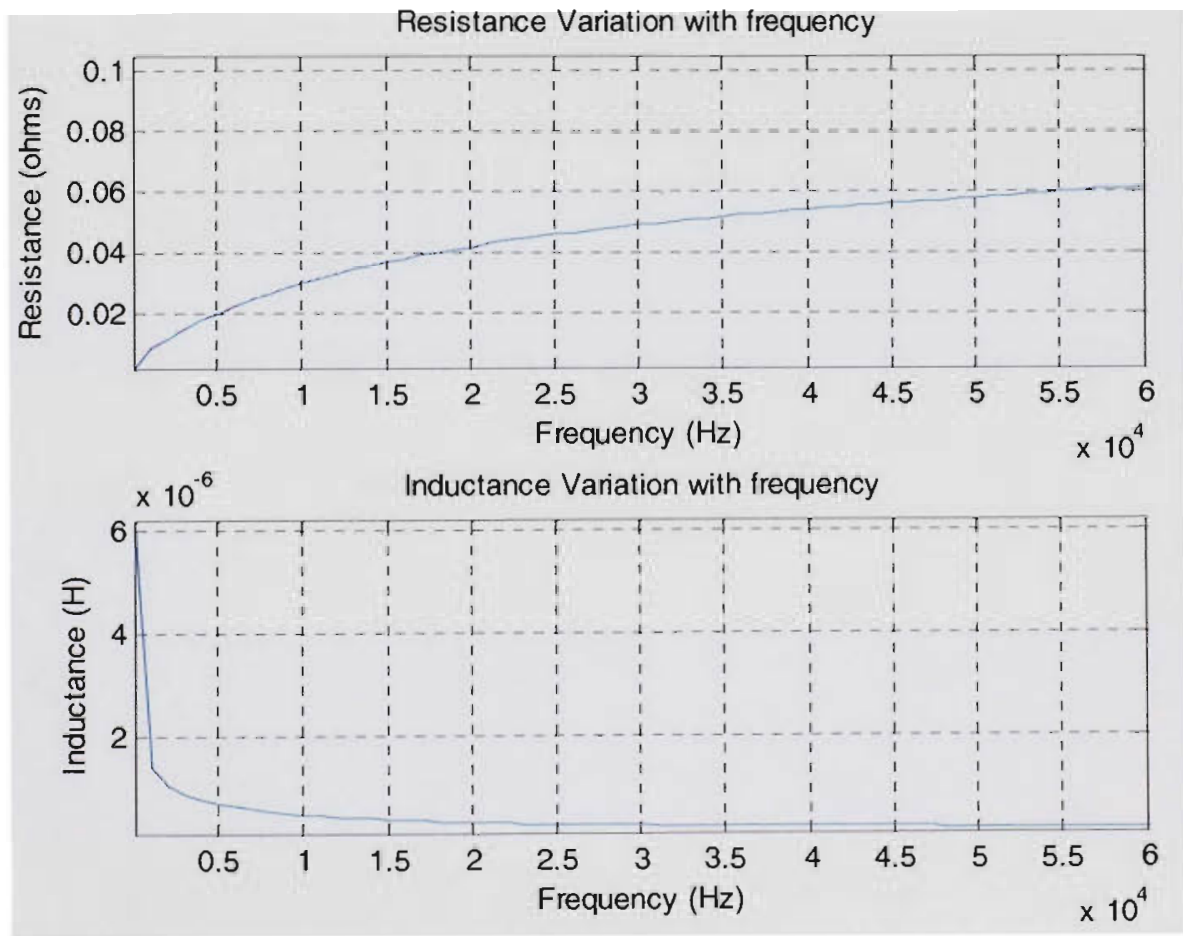

Fig.3.50. The triangle conductors cable resistance and inductance variation with the supply frequency 


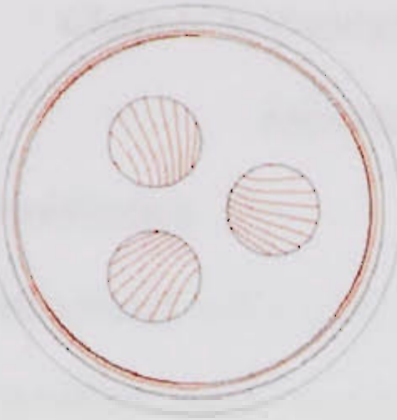

@ $60 \mathrm{~Hz}$

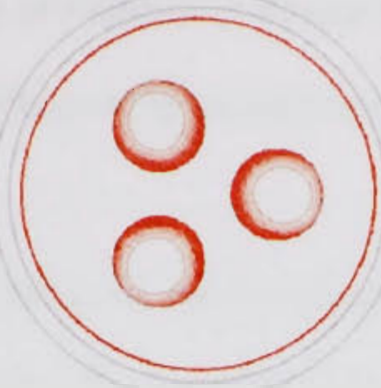

@8060 Hz

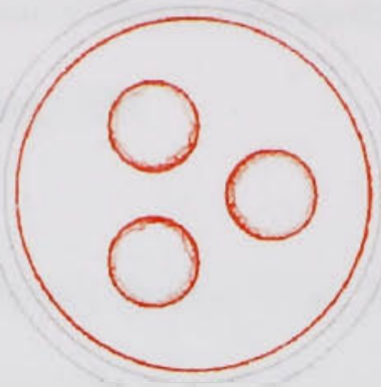

@100 kHz

Fig.3.51. The circle conductors cable current distribution at different frequencies

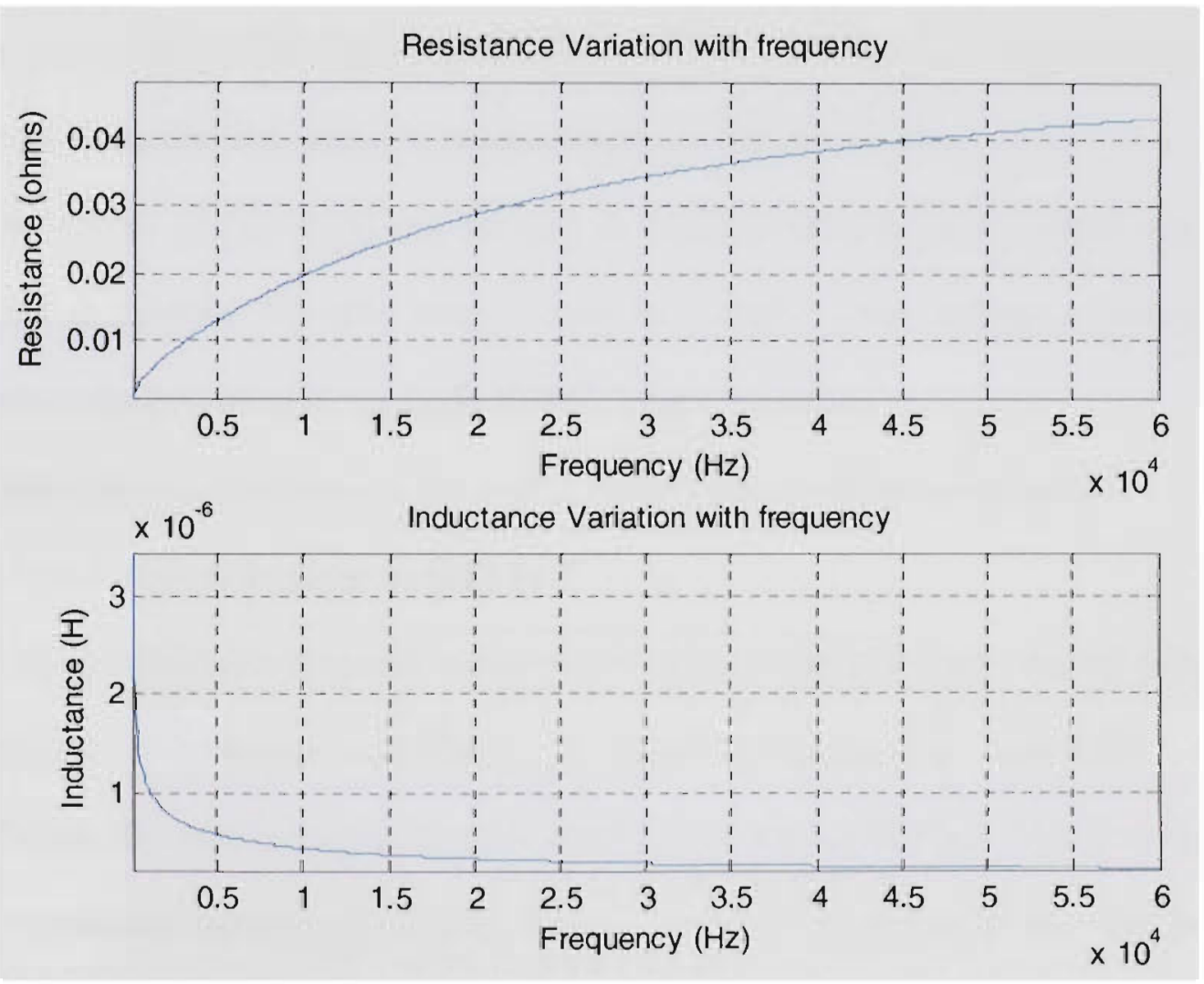

Fig.3.52. the circle conductors resistance and inductance variation with the supply frequency 


\section{Chapter 4: Simulation of Shipboard Power System Components under}

\section{Abnormal Operating Conditions Using FEM}

\subsection{Introduction}

This chapter deals with modeling of electromagnetic power devices under internal fault conditions using coupled-FEM. For a coupled-FE model, when a fault occurs inside the equipment, the magnetic flux distribution is fundamentally altered as well as the current in the circuit domain. But, the equipment terminal behavior still satisfies the coupled field circuit equations. Thus obtaining the faulty equipment behavior is achieved by solving these coupled equations.

This modeling approach includes the saturation effect, and other magnetic core non linear effects that usually ignored with other circuit based models. The circuit domain will provide us with the ability to generate faults at different instants.

The results of this study can be used to evaluate the protection system response, generate a database of fault cases, chose the suitable relay settings, optimize the equipment design and evaluate fault-tolerant control strategies.

\subsection{Finite element modeling of the power transformer with internal faults}

\subsubsection{Transformer model with internal fault}

Early detection and diagnosis of transformer internal faults are desirable for condition assessment, maintenance schedule and improved operational efficiency of the transformer. Transformer fault diagnosis requires accurate modeling of transformer under fault conditions, particularly internal faults. Nearly $70 \%$ of transformer failures are caused by undetected turn-to-turn faults. These faults usually begin as undetected turn-toturn faults in a coil, which progress to phase-to-phase or phase-to-ground short circuit 
faults. Because of costly repair, down time, and safety consideration, early detection of transformer internal faults is highly desirable.

The main objective of modeling transformers with internal fault is the lack of comprehensive field fault test databases. With internal faults the transformer modeling is more complex because the field picture totally changes. This in turn will affect the transformer parameters. Finite elements can be used to model the transformer under different internal faults and take into consideration its effect on the transformer parameters.

Most of the modeling methods for internal faults utilize a transformer pre-computed self and mutual inductances obtained from the short and open circuit test for the transformer or from the transformer manufacturer data. The faulty coil is divided into two or more subcoils according to the fault type, location, and number of turns involved, the self and mutual inductances between the newly created subcoils and the rest of the transformer coils are then evaluated by using the principles of consistency, leakage, and proportionality [58]. This technique assumes linear change in these parameters after the fault occurrence and therefore ignores the local saturation and leakage flux change due to the fault.

When a fault occurs, the distribution of the magnetic flux is fundamentally altered as well as the current is the circuit domain. But the electromagnetic field still satisfies the PDE of equations 2-9 and 2-13 and the current still satisfies the circuit equations 2-16. Thus obtaining the faulty transformer behavior is reduced to solving the three equations.

In order to simulate the internal faults geometry modification as well as circuit modification is necessary. The modification is based on the location of the internal fault, 
the number of turns involved in the internal faults, and type of the internal fault. The faulty coil is divided between two sub-coils, say ( $a$ and $b$ ) according to the fault location and number of turns involved. To simulate a turn to ground fault the transformer primary or secondary side the faulty coil is divided between two sub-coils coil (a and b) in the FE domain as well as in the circuit domain as shown in Fig. 4.1 a.

For turn to turn fault the faulty winding was subdivided into three subcoils $(a, b$, and c) in the FE domain as well as in the circuit domain. A turn to turn fault was also imposed by short circuit the two coils ( $\mathrm{a}$ and $\mathrm{b}$ ) in the circuit domain by using a time control switch as shown in Fig. 4.1 b.

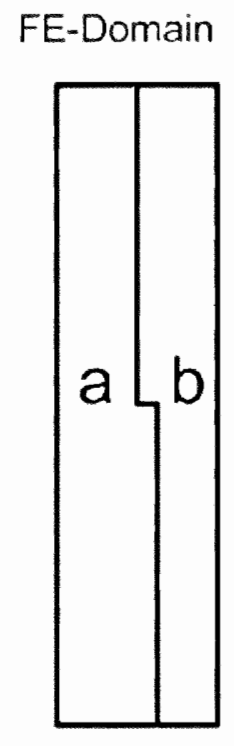

a
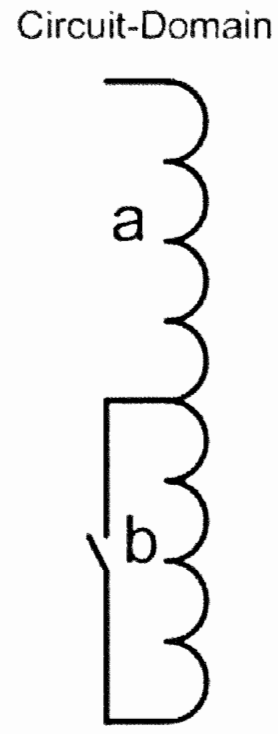

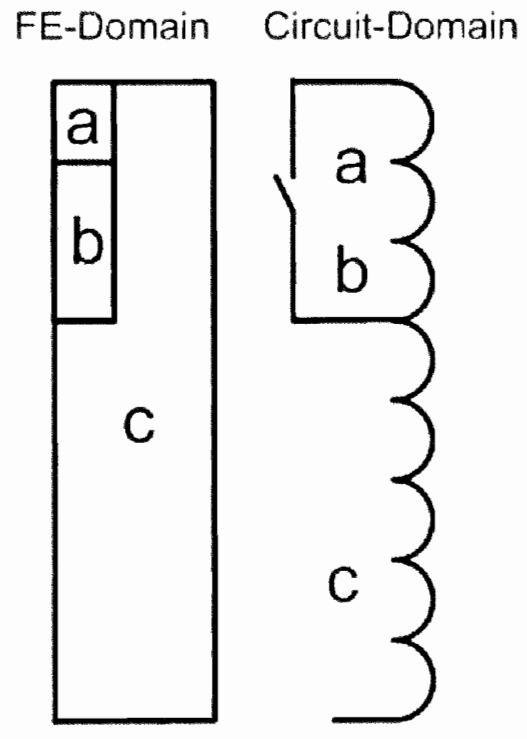

b

Fig.4.1. FE domain and circuit domain representation of the transformer primary coils for

(a) turn to ground Fault (b) turn to turn Fault

A time control switch was utilized to generate the fault in the circuit domain as shown in Fig. 4.1 at the desired time. Figure 4.2 shows the circuit model of the whole system with transformer, source, and the non-linear load. 
The transformer behavior with internal fault condition was investigated under sinusoidal source and nonsinusoidal source. Thus we will consider the effect of supply harmonics on the fault features.

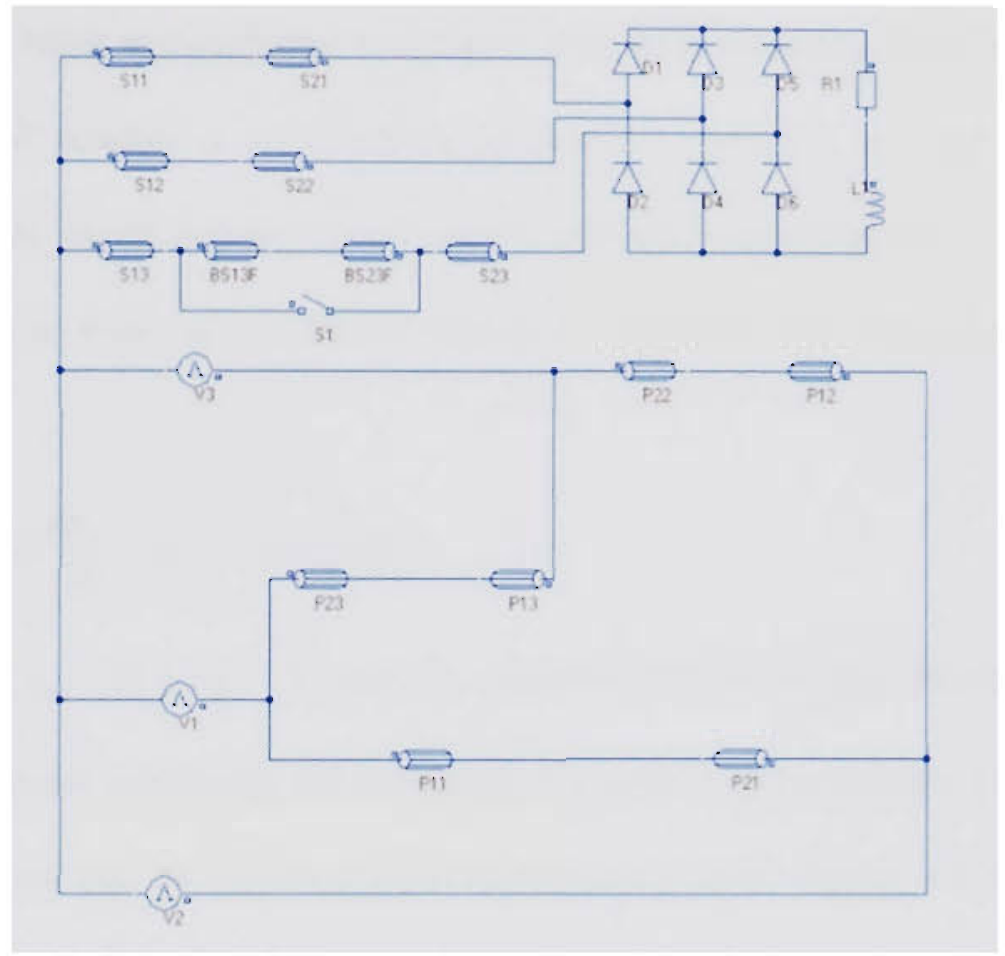

Fig.4.2. Circuit model of the transformer with internal fault on its secondary side, source and nonlinear load

\subsubsection{Transformer model with arcing internal fault}

An Arcing fault is a form of short circuit condition with a fault current magnitude that varies according to the fault conditions. The term "arcing" is defined as a luminous conduction of electricity across an insulating medium usually air, and usually cause a damage that is limited to the fault area. The conduction takes place when the electric field intensity exceeds the insulation material breakdown strength (Around $30 \mathrm{kV} / \mathrm{cm}$ for air). Arcing faults have deadly thermal and mechanical consequences on the transformer; the 
resulting temperature may reach several thousand degrees Celsius and may cause conductors at the fault point to melt and may lead to a fire [98].

The arc model describes the non-linear relationship between the arc voltage and the arc current [95]. Most arc modeling techniques start with assuming a big resistance and advanced until it reaches a combination of resistor, inductor, voltage sources, and capacitors. The arc model parameters are usually obtained experimentally.

We utilized in our study an arc model that is described by the following differential equation [95]:

$$
V_{\text {arc }}=R i_{a r c}+L \frac{d i_{a r c}}{d t}+\left(C_{1}+C_{2} \times g\right) i_{a r c}^{0.12}
$$

Where, $V_{\text {arc }}, i_{\text {arc }}, \mathrm{R}, \mathrm{L}, \mathrm{g}, C_{1}$ and $C_{2}$ represent the arc voltage, the arc current, the arc resistance, the arc resistance, the arc length, $\mathrm{C} 1$ and $\mathrm{C} 2$ are constants. The magnitude of these parameters can be obtained from experimental tests. Figure 4.3 shows the arc equivalent circuit.

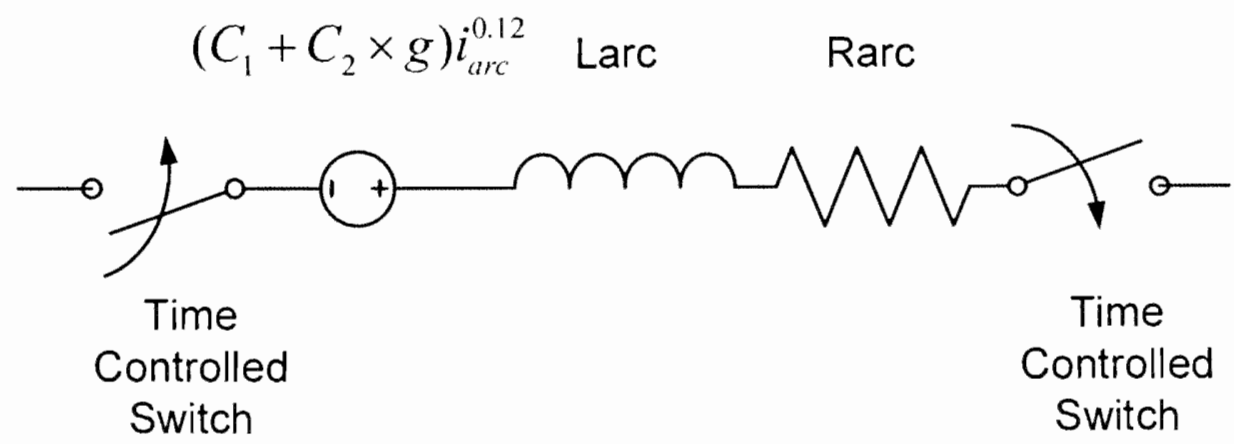

Fig.4.3. The arc equivalent circuit

The arc was incorporated in the transformer FE model by indirect coupling between FEM model and Simulink implementation. During the time-stepping simulation, the 
variables were passed directly back and forth between the FEM and arc Simulink model.

Fig. 4.4 shows an outline of the coupling between FEM and Simulink.

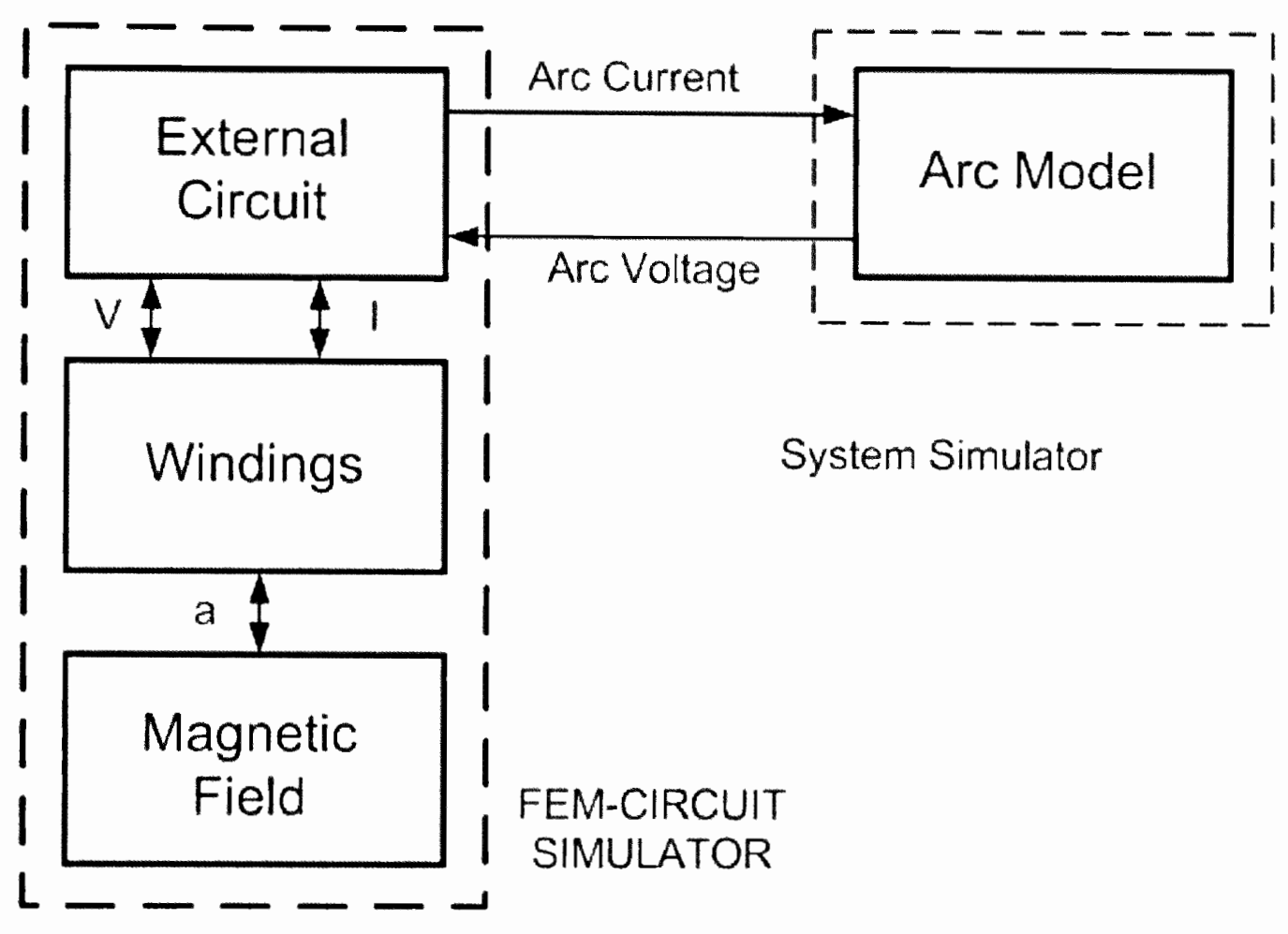

Fig.4.4. Outline of coupling between FEM and the arc model

\subsubsection{Simulation results}

Six different internal fault types were simulated: turn-to-ground fault on the phase c primary side, turn-to-ground fault on the phase c secondary side, turn-to-turn fault on the phase c primary, turn-to-turn fault on phase c secondary, arcing turn-to-ground fault on the primary side, and arcing turn-to-turn fault on the phase $\mathrm{c}$ primary side winding.

The fault was initiated at 0.0333 second. Figure 4.5 shows the magnetic flux distribution inside the transformer at $\mathrm{t}=0.1884 \mathrm{sec}$ for normal operation, turn-to-turn fault, and turn-to-ground fault on the transformer phase $\mathrm{c}$ secondary windings under PWM supply. Figure 4.6 shows the magnetic flux distribution inside the transformer at 
$\mathrm{t}=0.1884 \mathrm{sec}$ for turn-to-turn fault, and turn-to-ground fault on the transformer phase "c" primary side windings under sinusoidal supply. In these figures, the flux line circulating around the coils is the leakage flux. It's clear that the magnetic flux distribution is fundamentally altered during these faults. There is a large radial component of leakage flux around the shorted turn.

For an internal fault on the primary winding of phase $\mathrm{c}$, either it is a turn-to-ground or turn-to-turn fault, there will be a current increase in phase $\mathrm{c}$ and phase a and a decrease in phase $b$ current while their secondary winding current's does not change. The percentage increase in the primary current is higher in case of turn-to-ground fault.

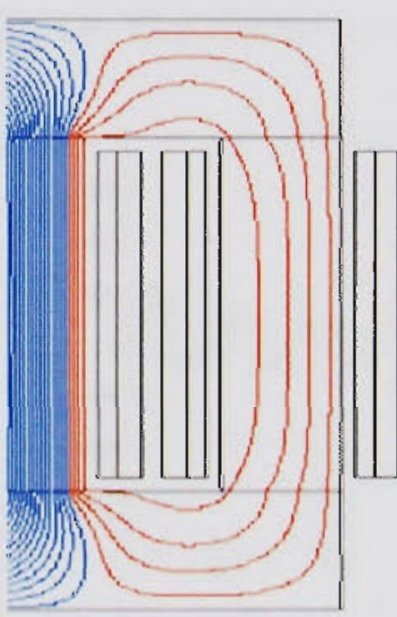

Vormal Operation

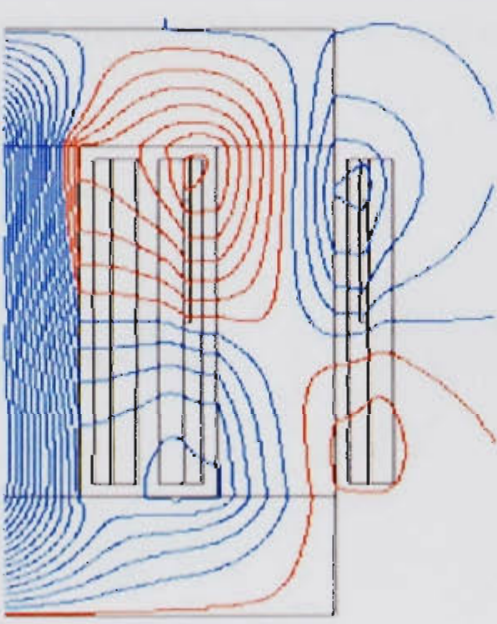

Turn-to-Turn Fault

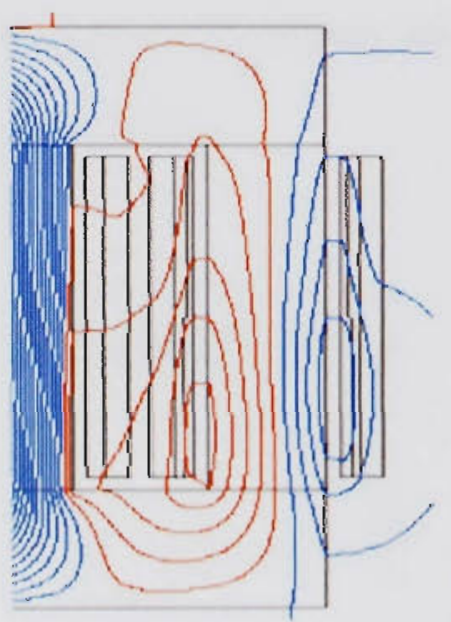

Turn-to-Ground Fault

Fig.4.5. Transformer magnetic flux distribution for different cases on the secondary side

Figure 4.7 and Fig. 4.8 shows the transformer three phase primary currents and DC side for currents turn-to-ground and arcing turn-to ground under PWM supply.

Figure 4.9 shows the transformer three phase primary and DC side currents for turnto-turn, and arcing turn-to-turn faults on the primary side under PWM supply. Furthermore, the transformer secondary winding fault on phase c, either it is a turn-to- 
ground or turn-to-turn fault, the primary currents in phase a and phase $\mathrm{c}$ will increase while the primary current of phase $b$, the secondary currents and the dc side current will decrease.

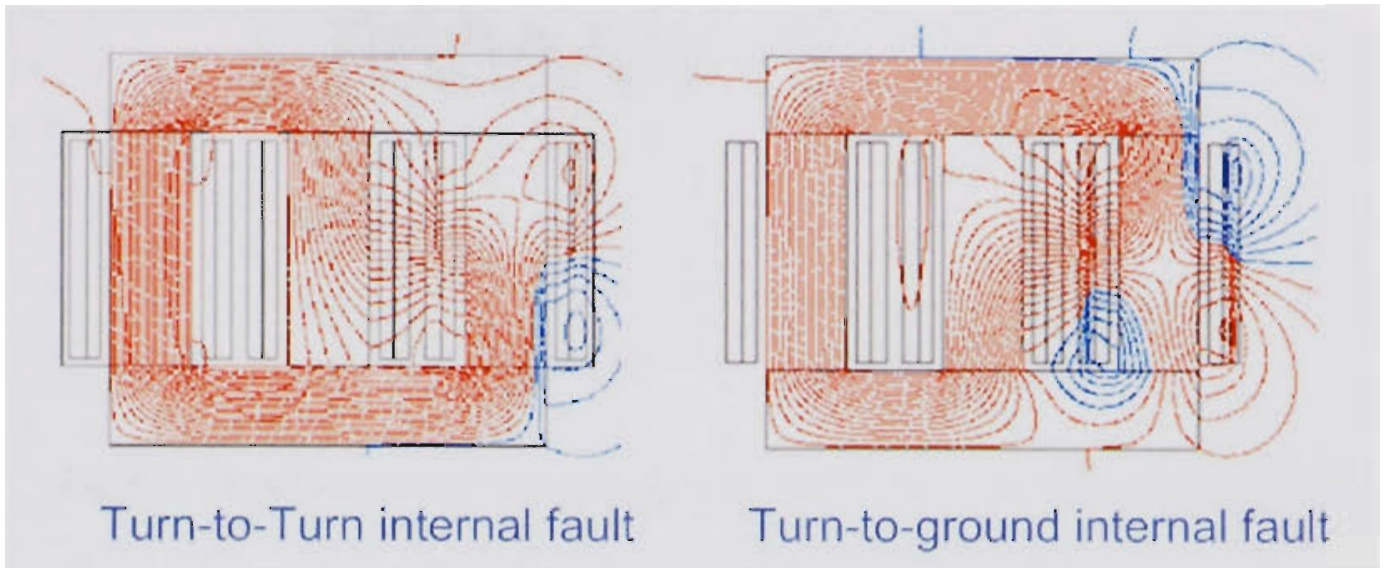

Fig.4.6. Transformer magnetic flux distribution for different cases on the primary side
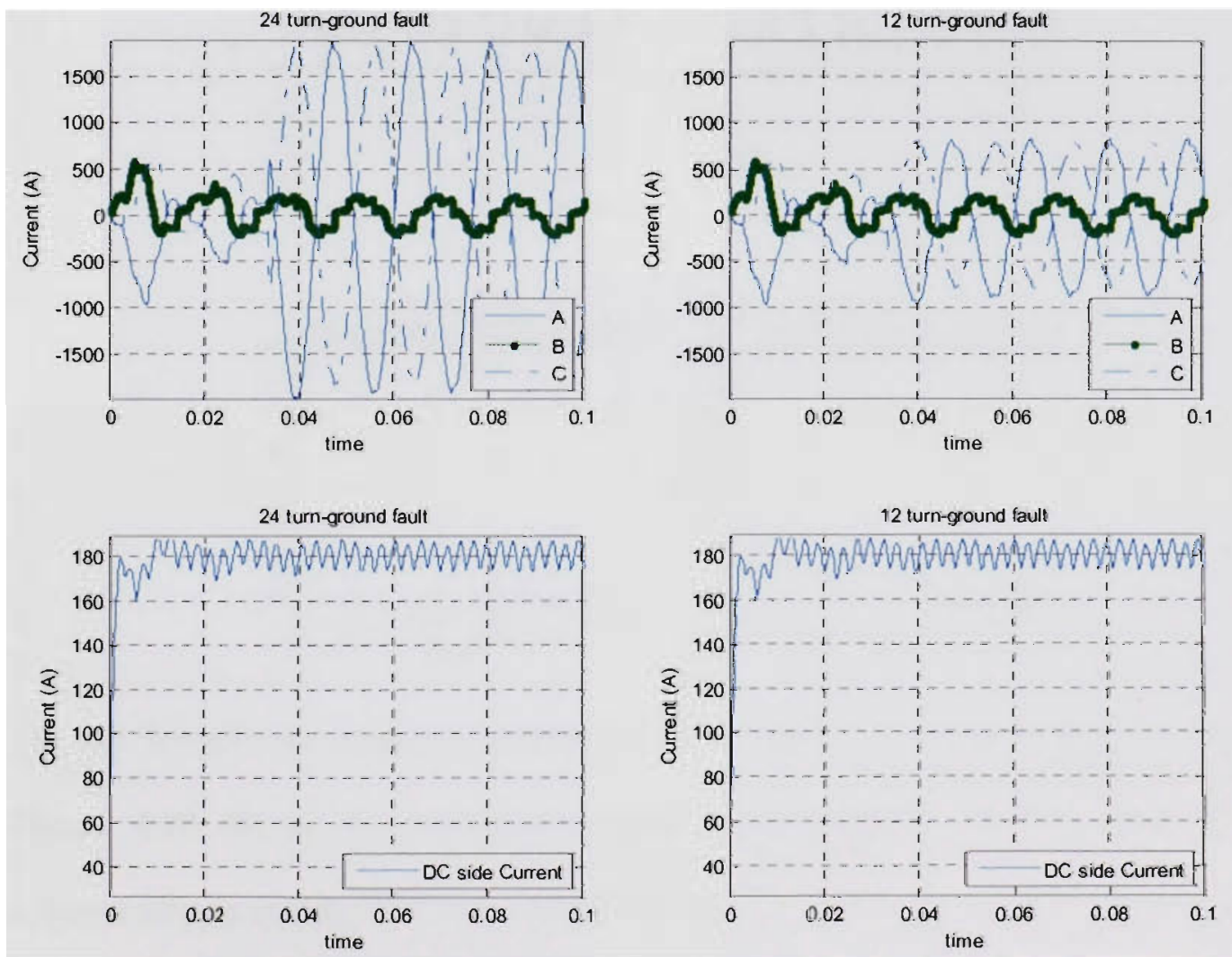

Fig.4.7. Transformer primary currents and the DC side for turn-to-ground faults 


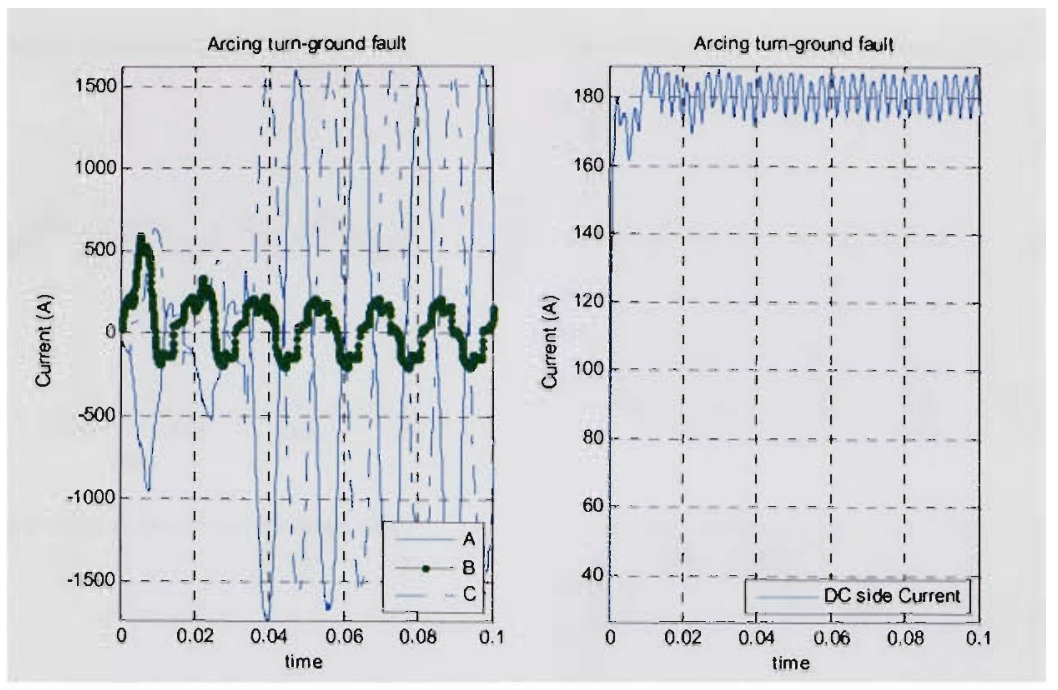

Fig.4.8. Transformer primary currents and the DC side for different arcing faults
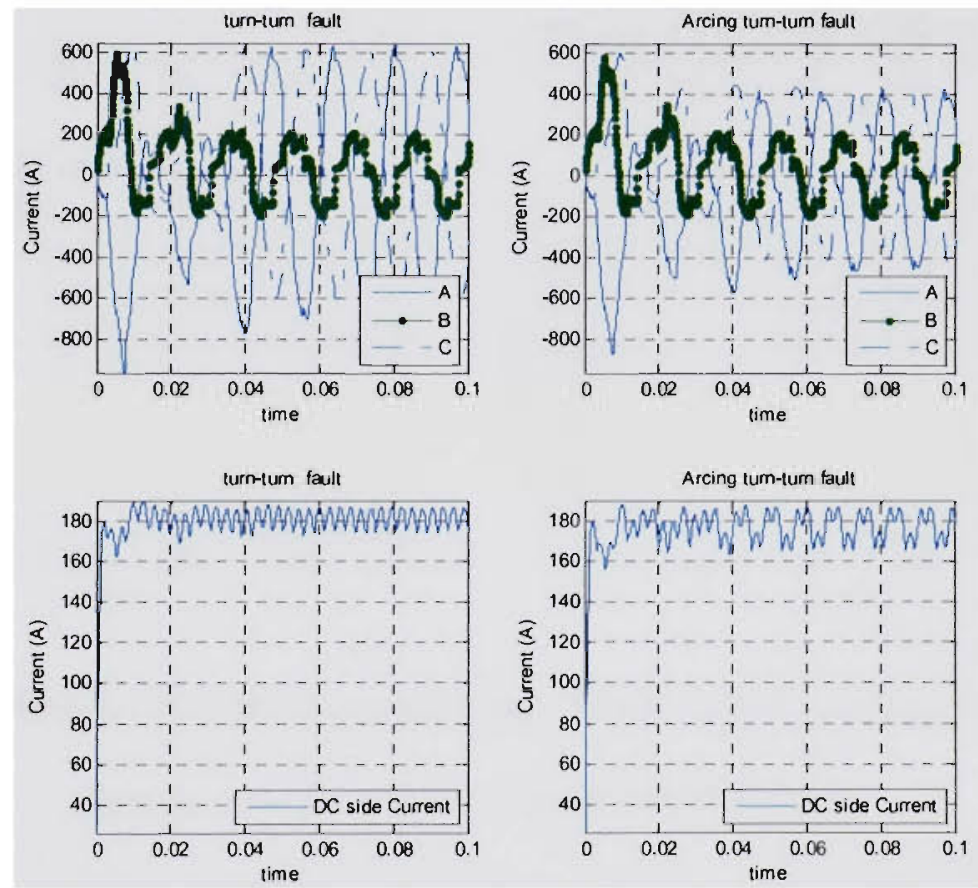

Fig.4.9. Transformer primary currents and the DC side for different turn-to-turn faults

Figure 4.10 shows the transformer three phase primary currents, three phase secondary currents and the DC side current for turn-to-ground and turn-to-turn faults on the transformer phase c secondary side under PWM supply. 

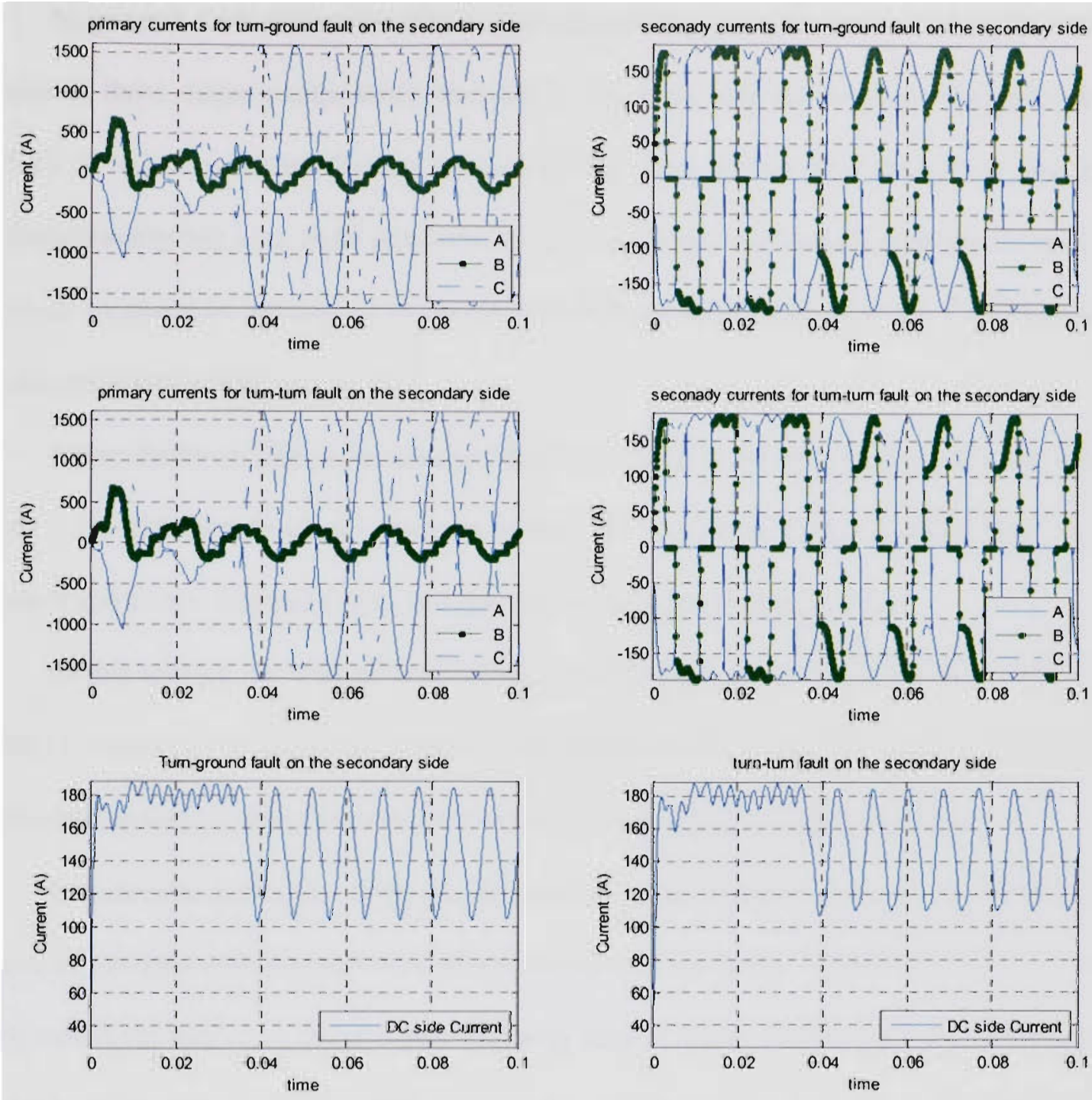

Fig.4.10. Transformer three phase primary, secondary, and DC currents for secondary turn-to -ground and turn-to-turn internal faults

\subsection{Finite element model of the three phase induction machine with internal fault}

\subsubsection{Introduction}

Induction motors are widely used in many industrial processes because they are cost effective and mechanically robust. Because of costly machinery repair, down time, and safety consideration, early detection of motor internal faults is highly desirable. 
In general, fault diagnosis of induction motors has concentrated on sensing failures in one of three major components, the stator, the rotor, and the bearings. Approximately $36 \%$ of induction motor failures are caused by failure of the stator windings, and these faults usually begin as undetected turn-to-turn faults in a coil, which progress to phase-tophase or phase-to-ground short circuit faults. Rotor failures account for $5-10 \%$ of total induction motor failures [84-86].

Stator faults are the main cause of induction motors outages. These faults are caused by four different types of stresses acting on insulation system, mechanical stress, electrical stress, thermal stress, and the surrounding environment [84].

In this section we will address broken squirrel-cage bars fault, and stator turn-to-turn faults. Modeling of induction motors with internal faults is the first step in the design of the fault detection algorithms and the evaluate fault tolerant control strategies.

For internal faults the modeling situation is more complex because the field picture totally changes which in turn will affect the motor parameters. Finite element can be used to model the induction motor under different internal faults. FEM takes into consideration the local change of the magnetic permeability due to the slotting effect. The field-circuit coupling allows us to simulate the operating conditions of the induction motor with the real power supply connections.

\subsubsection{The FE model}

Unlike the normal operation model the geometry is not symmetrical because of the fault, so the full geometry has to be used. The finite element mesh of the induction motor consisting of 43721 nodes forming 21822 second order elements as illustrated in Fig. 4.11 . 
In order to simulate the internal faults geometry modification as well as circuit modification is necessary. The modification is based on the location of the internal fault, and the number of turns involved in the internal faults. Stator turn to turn fault is caused by an insulation breakdown between two turns. To simulate a stator turn to turn fault, coils representing the faulty turns are short circuited by using a time control switch in the circuit domain as shown in Fig. 4.12. The circuit domain representation of the induction motor with internal fault is shown in Fig. 4.13.

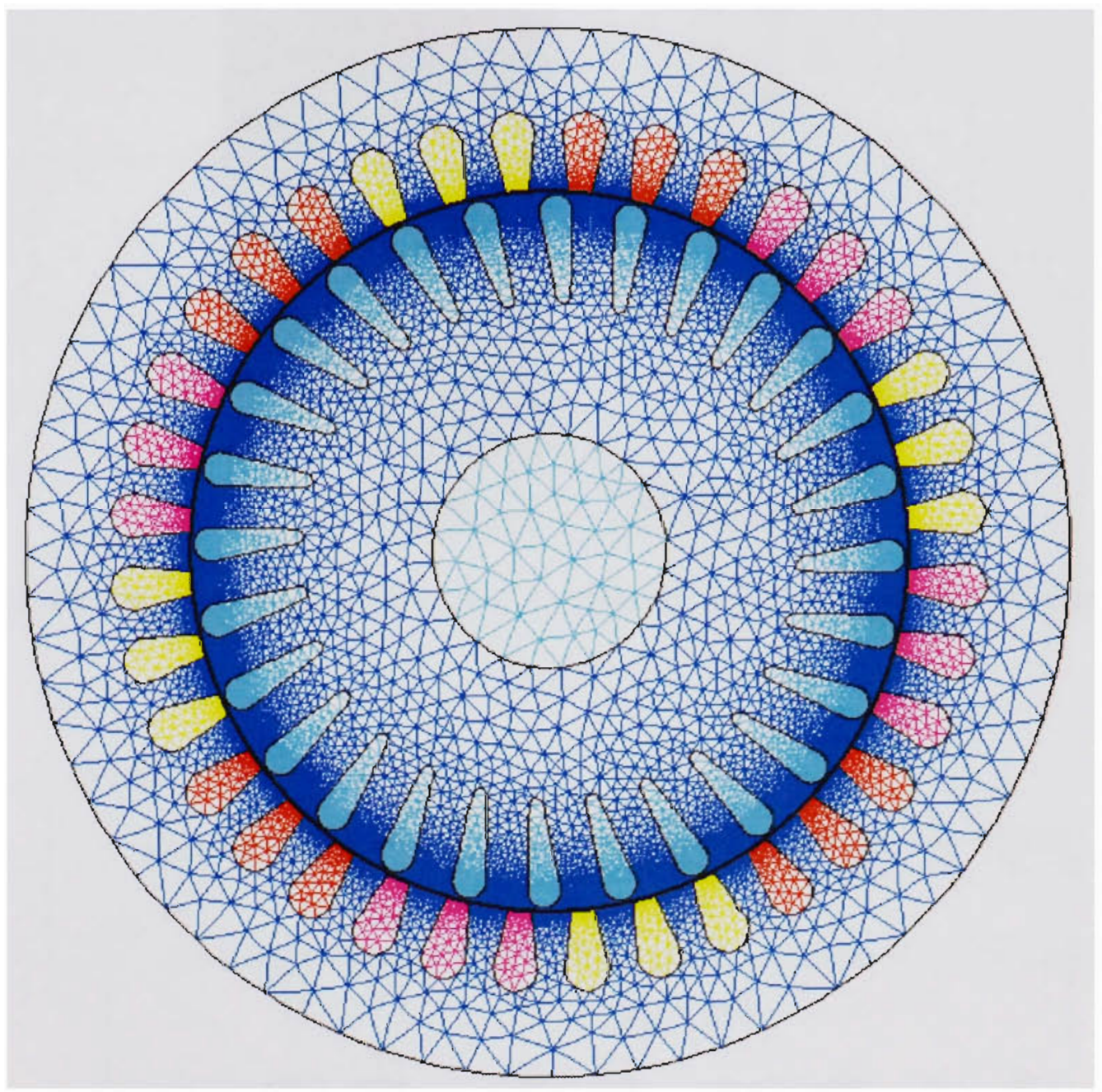

Fig.4.11. Finite element mesh of the induction motor 
The broken bars are realized by assigning high resistivity to the broken bars. The fault severity is changed by varying the number of broken bars and the number of stator shorted turns. A combination of the two faults is also investigated. The behavior of induction motor with internal fault was investigated under sinusoidal source and PWM source.

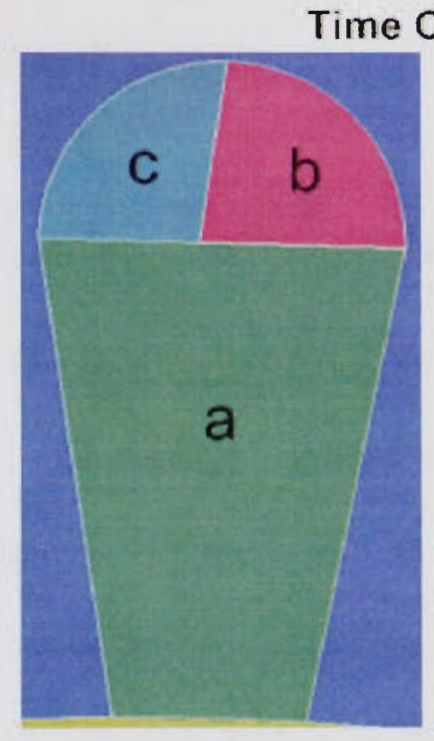

FE Domain

\section{Time Control Switch}

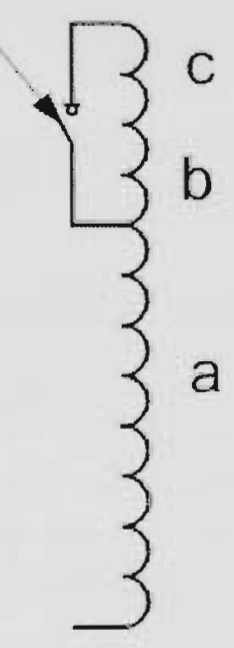

Circuit Domain

Fig.4.12. FE and circuit domain representation of the induction motor turn to turn internal fault

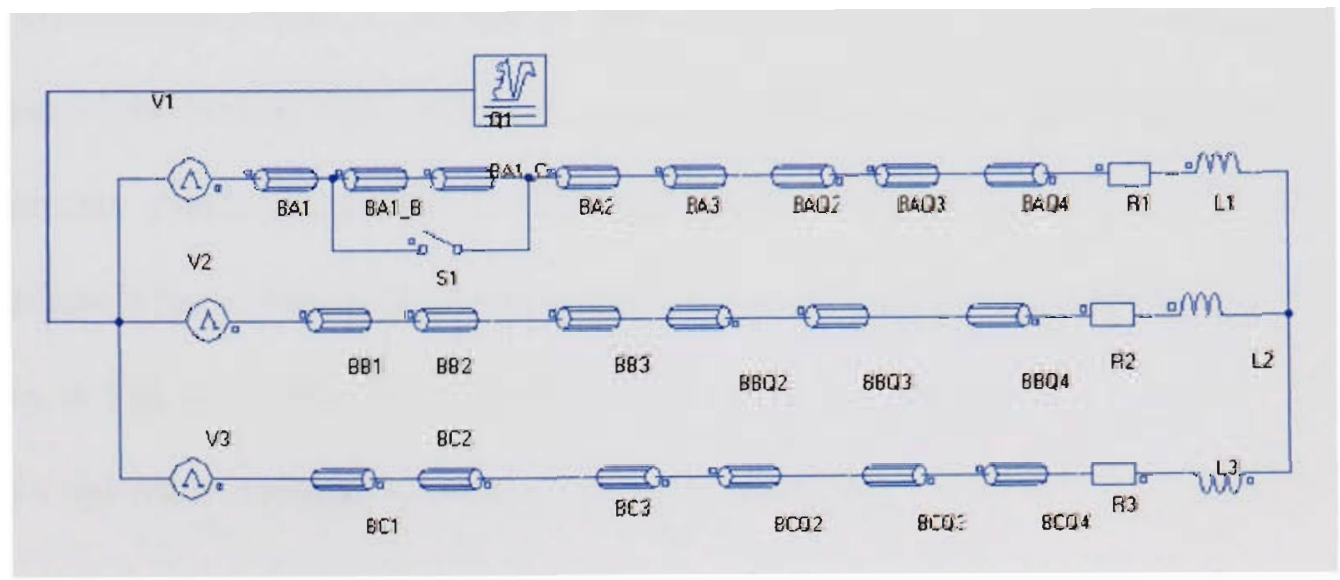

Fig.4.13. Circuit model of the Induction motor with stator internal fault 


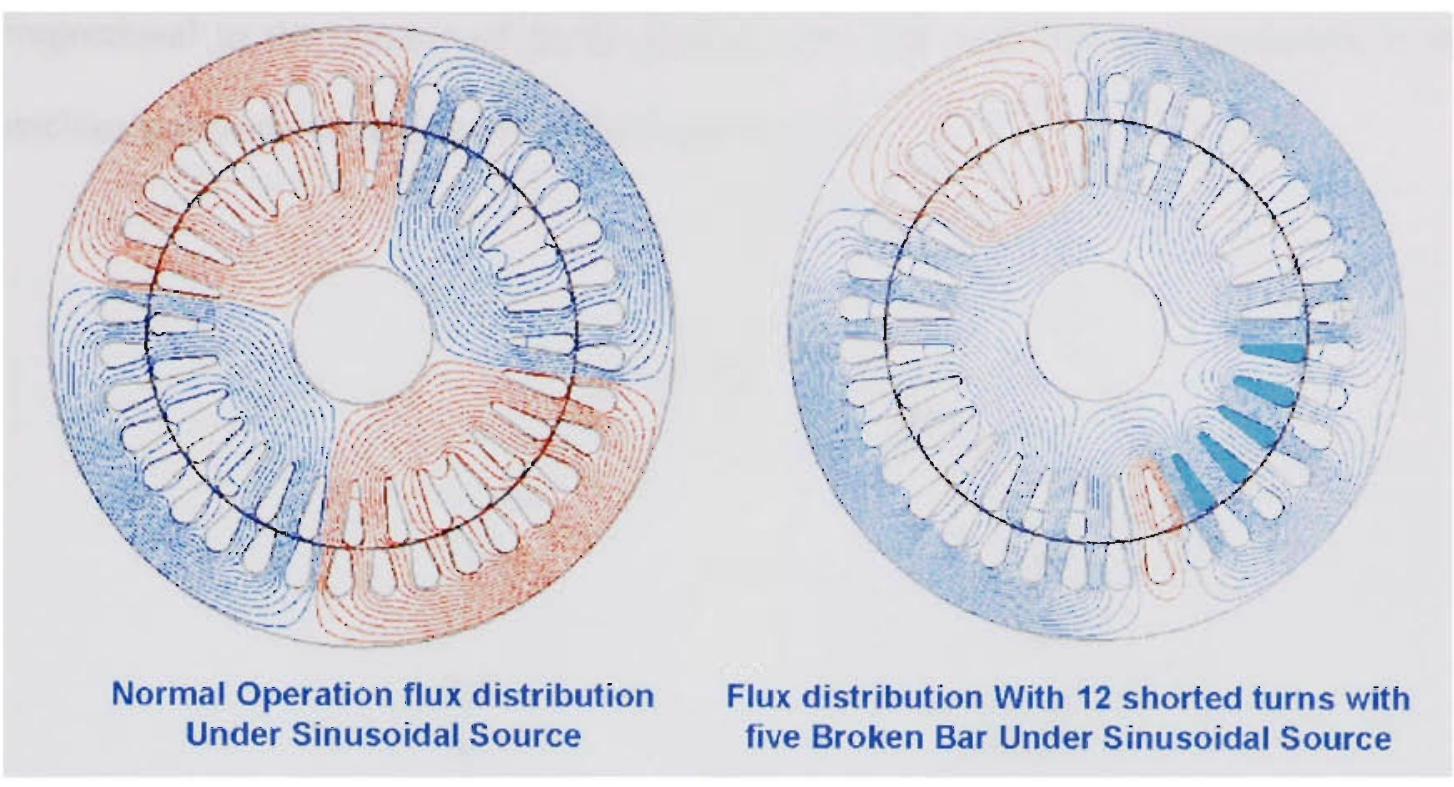

Fig.4.14. Magnetic flux distribution for normal operation and for 12 shorted turns with five broken bars under sinusoidal source

\subsubsection{Simulation results}

The magnetic flux distribution is fundamentally altered during internal faults. Figure 4.14 shows the magnetic flux distribution for healthy motor and for 12 shorted turns with five broken bars motor under sinusoidal voltage source.

In the case of broken rotor bars, the magnetic field is no longer sinusoidal around the air-gap circumference due to the lack of induced currents in the broken bars. The high resistance of the broken bars will cause unsymmetrical rotor currents distribution. The rotor currents which are unable to flow through the broken bars are flowing in the adjacent bars. This increases the currents flowing through the bars next to the broken bars as shown in Fig. 4.15. This will lead to thermal stress in these bars. It should be noted that the total rotor currents is reduced due to broken bars fault and the reduction is 
proportional to the number of faulty broken bars. This will lead to degradation in the machine performance and increase the toque ripples.

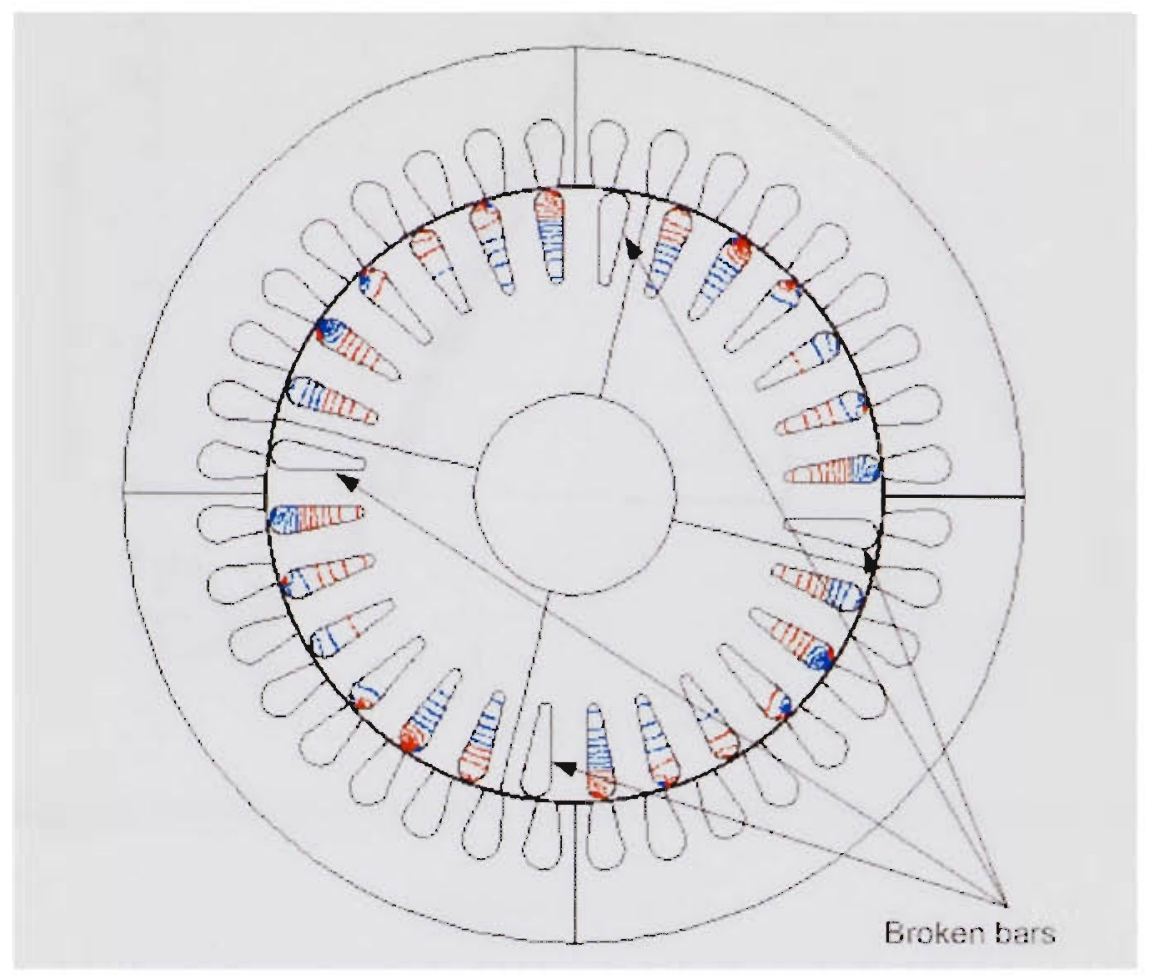

Fig.4.15. Current distribution in the induction machine bars under broken bars fault

Also, in the stator and rotor teeth near broken bars there is an increase in the magnetic flux density which gives rise to local saturation, which will affect the motor harmonic behavior. In the case of stator shorted turns fault the air gap vector magnetic potential field is no longer symmetrical.

Figure 4.16 and Fig. 4.17 show the magnetic vector potential in the air-gap for different internal faults under sinusoidal and non-sinusoidal supply.

Figure 4.18 shows the induction machine torque for healthy motor, four broken bars, and stator with 12 shorted turns under sinusoidal voltage supply. It can be observed from the graph that the internal faults effect on the machine in the transient and steady state is very noticeable. 


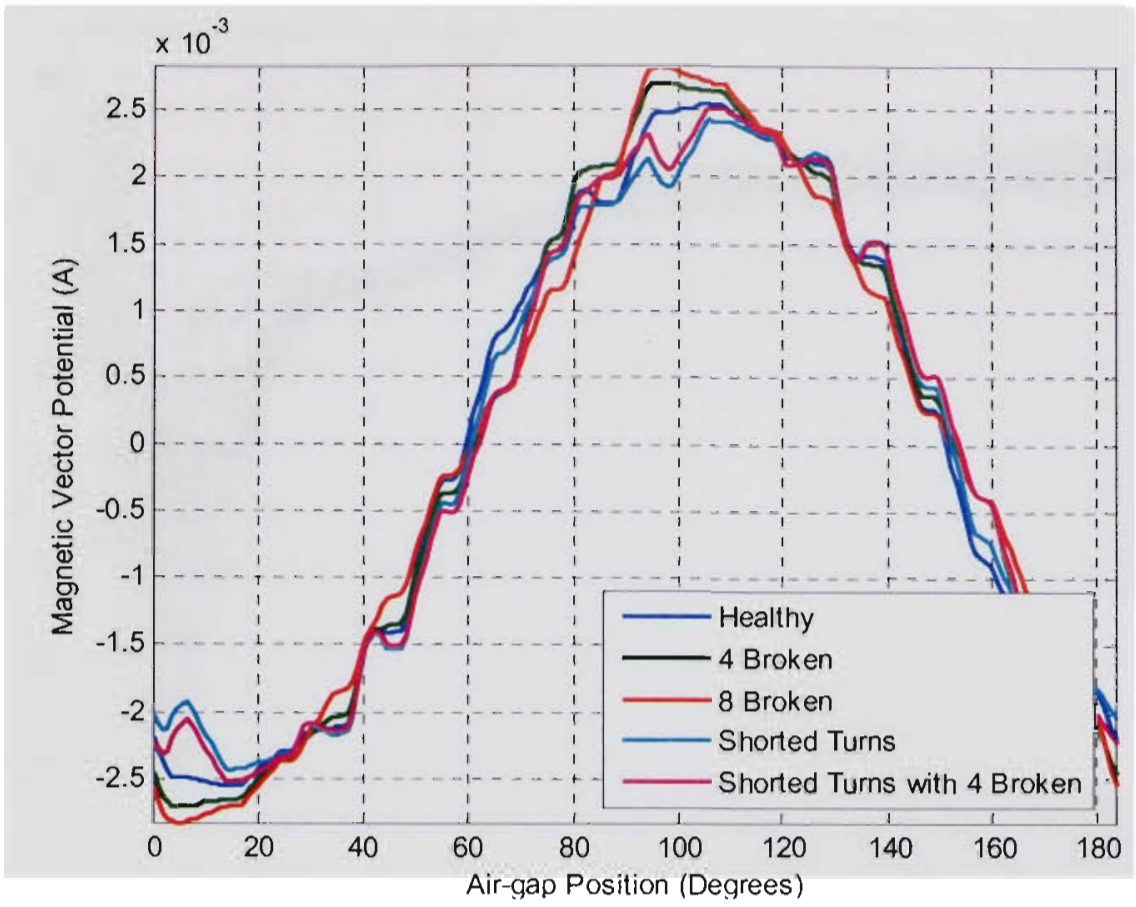

Fig.4.16. Magnetic vector potential in the middle of the air-gap for different cases under sinusoidal supply

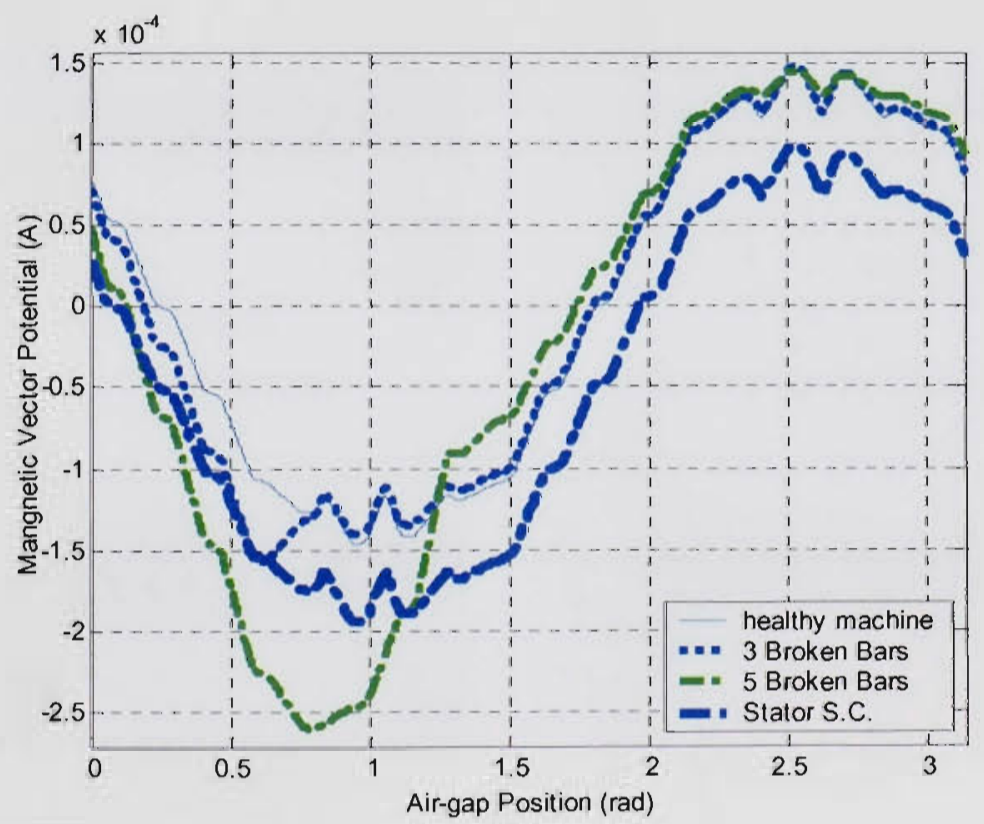

Fig.4.17. Magnetic vector potential in the middle of the air-gap for different cases under non-sinusoidal supply 


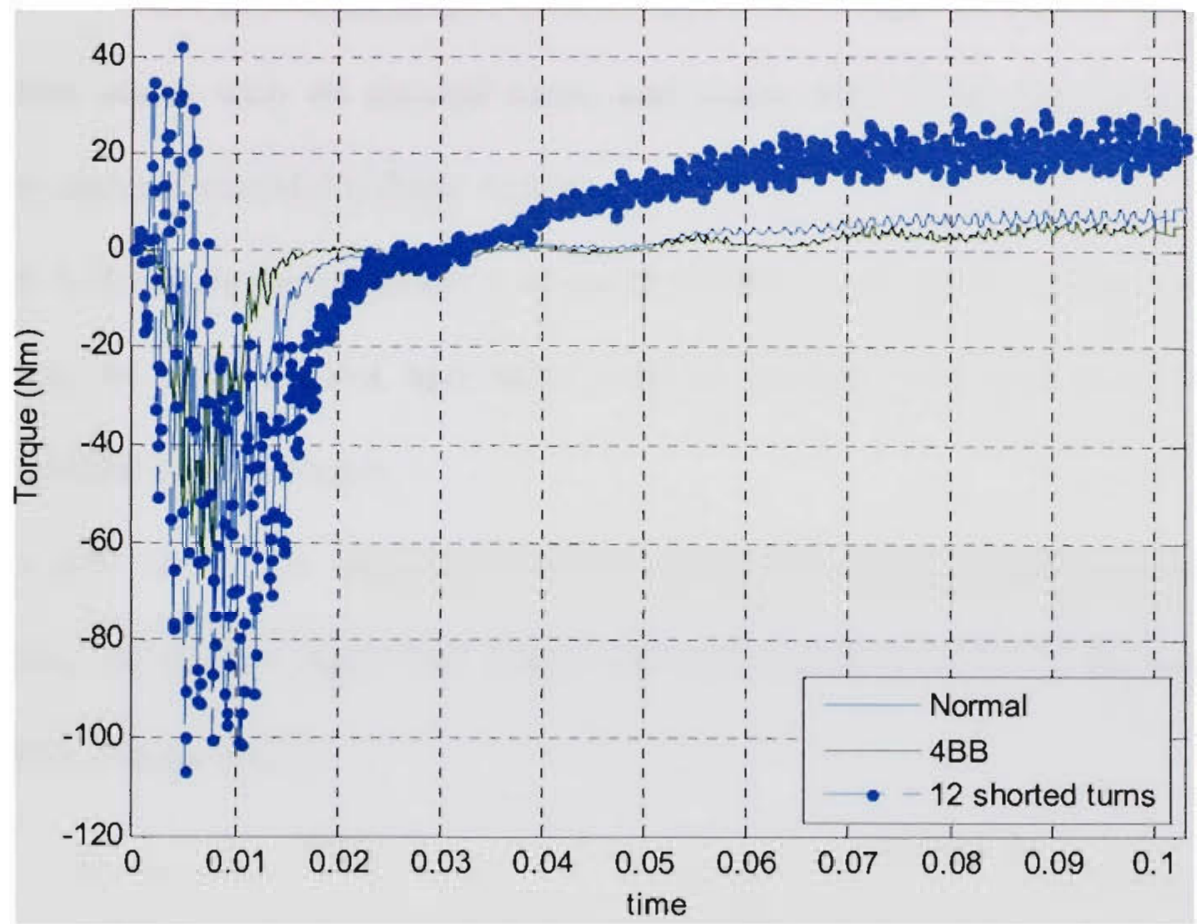

Fig.4.18. Induction motor torque profile for different internal faults
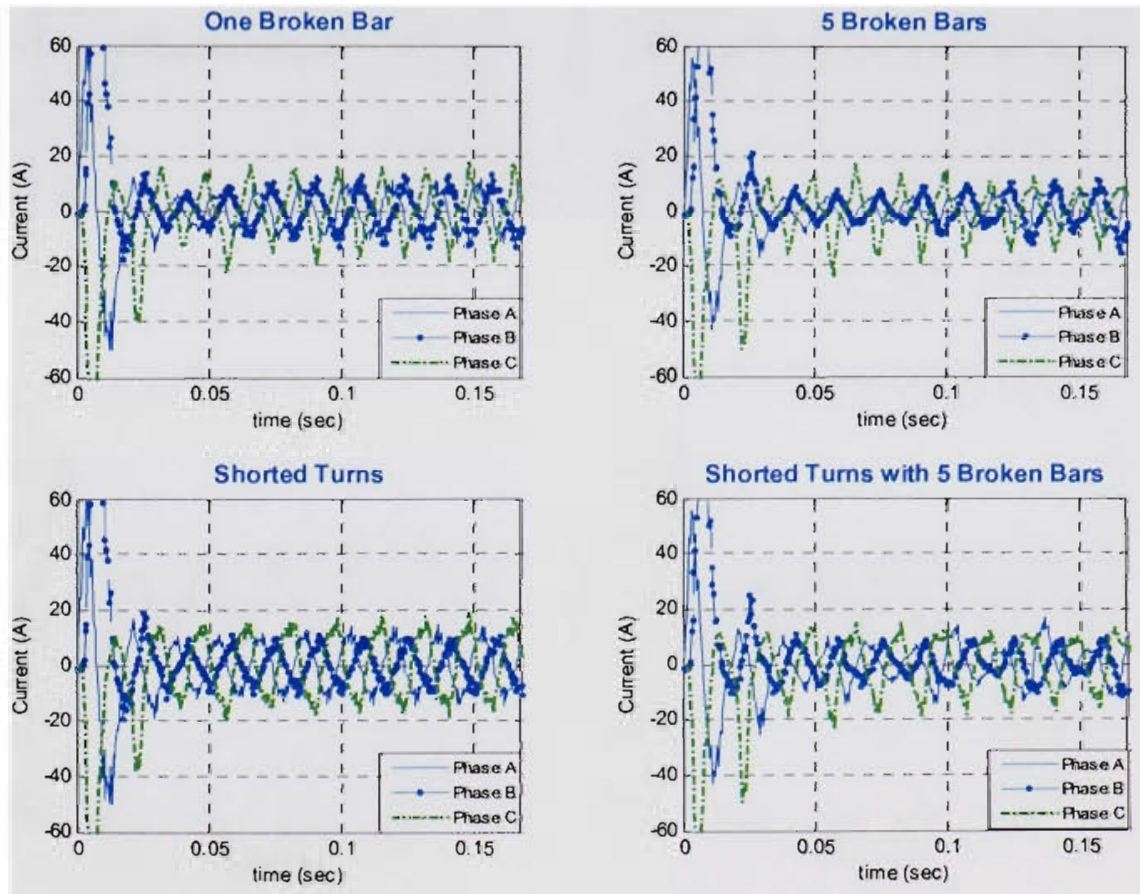

Fig.4.19. Induction machine phase a current for different internal faults under sinusoidal source 
Figure 4.19 shows the induction machine phase a current for one broken bar, five broken bars, stator with 44 shorted turns, and stator with 44 shorted turns with five broken bar under sinusoidal voltage supply.

Figure 4.20 shows the induction machine three phase currents for one broken bar, five broken bars, 44 shorted turns, and stator with 44 shorted turns with five broken bars under sinusoidal voltage supply.

Figure 4.21 shows the induction machine three phase currents one broken bar, five broken bars, 44 shorted turns, and stator with 44 shorted turns with five broken bars under PWM voltage supply.
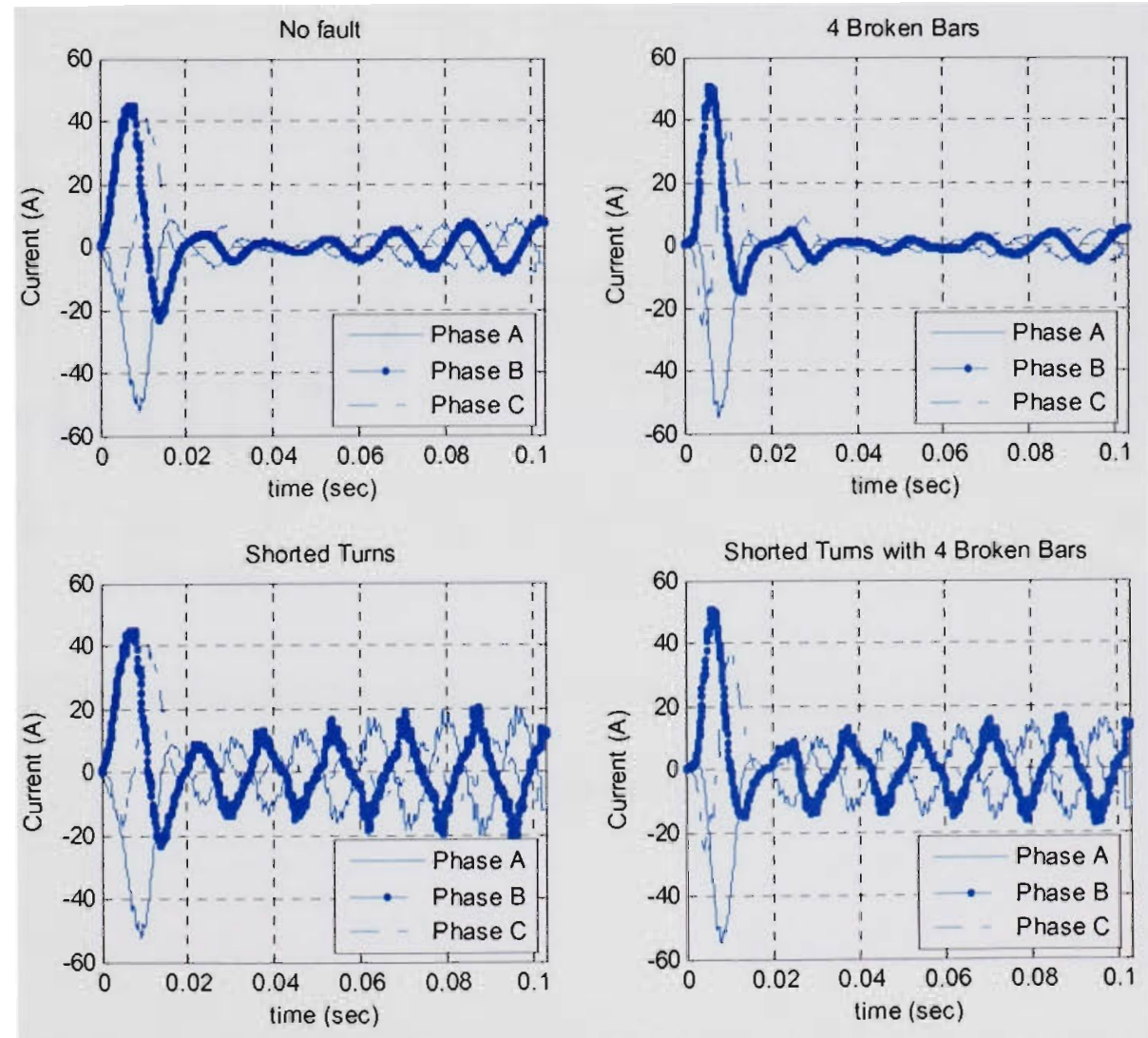

Fig.4.20. Induction machine three phase currents for different internal faults under sinusoidal source 


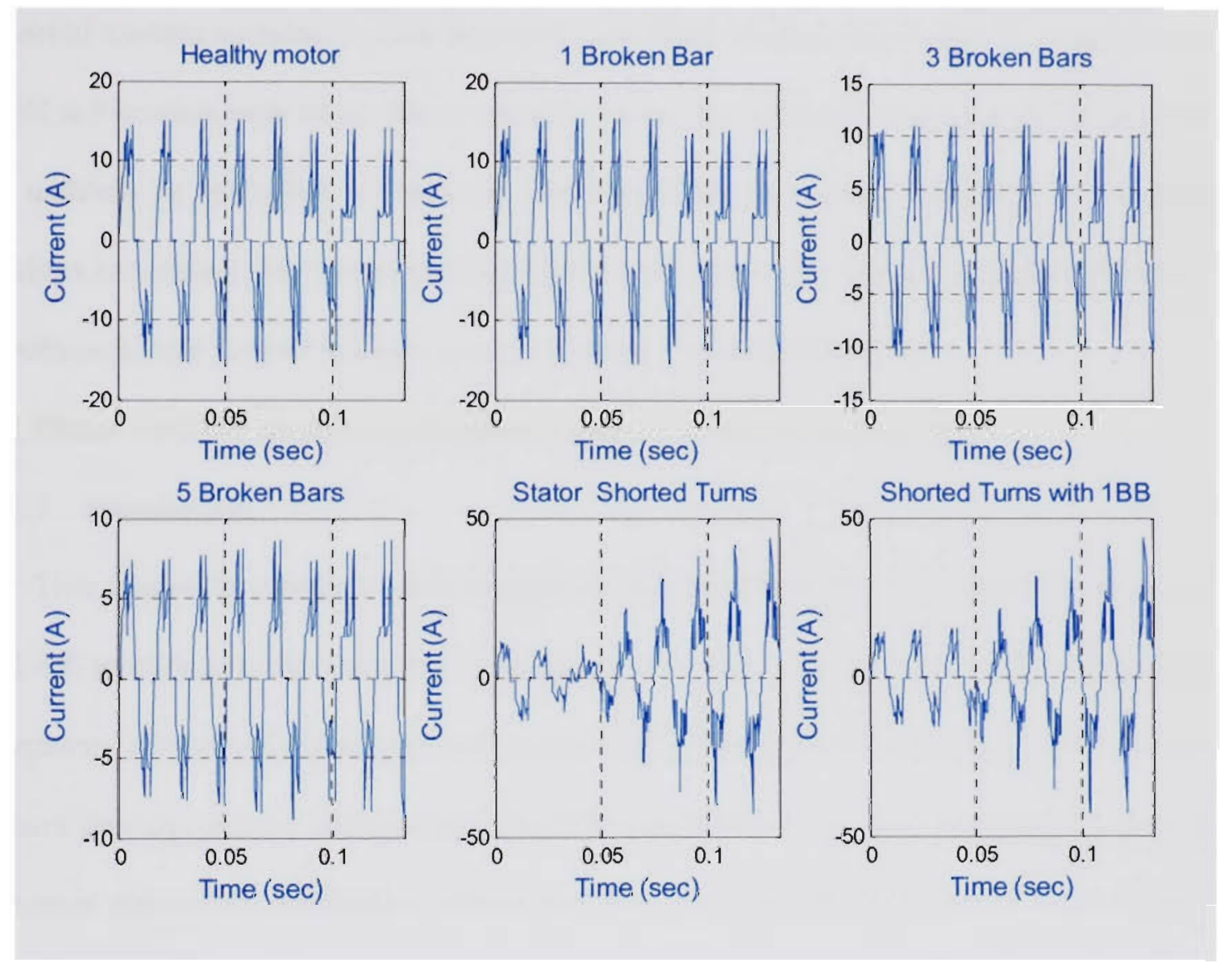

Fig.4.21. Induction machine phase a current for different internal faults under nonsinusoidal source 


\section{Chapter 5: Modeling of Shipboard Power System Components for Low}

\section{Frequency Studies}

\subsection{Introduction}

This chapter deals with modeling of different electromagnetic power devices in the selected system simulator. This approach use FEM models developed in chapter 3 to build a Simulink equivalent phase variable model for different electromagnetic devices. In addition to Simulink we utilized SimPowerSystems toolbox which is a Simulink toolbox to conduct our system studies. SimPowerSystems can be used to model electrical, mechanical, and control systems in one simulation environment [27].

\subsection{Phase variable model of permanent magnet synchronous machine}

\subsubsection{Introduction}

This modeling technique was suggested and developed by O.A. Mohammed et al. [11-19] to overcome the d-q model inaccuracy especially when considering the switching frequency effects such as current and torque ripple. The $d-q$ model for electrical machines ignore the core related effects and their effect on the machine operational parameters. It assumes sinusoidal distribution of air gap flux density and obviously all the derived waveforms from it. Despite, the ability of the d-q model to accurately predict the motor current fundamental component and the average value of torque near nominal operating conditions, the model accuracy deteriorates at other operating points far from the nominal operating conditions [96].

The conventional $\mathrm{d}-\mathrm{q}$ motor model typically used in electric drive simulations is inaccurate in predicting the machine behavior, except for the current fundamental component and the average torque near rated operating conditions. Predictions of current 
and torque ripple are often in error by a factor of two to five [96]. Furthermore, d-q model becomes mathematically inaccurate when the symmetry of mutual inductances is removed for some specific design considerations. This is certainly the case as we increase the number of poles in the machine to increase power density but in the process have a small number of slots per pole per phase. Also during the transient conditions when stator and rotor mmfs are not constant in magnitude or in angle with respect to each other then harmonic components are generated in the electrical and mechanical system. These harmonic components can not be described accurately using d-q frame of reference.

Therefore a new modeling technique was introduced called the phase variable model to represent the machine in actual three phase reference frame instead of regular $\mathrm{d}-\mathrm{q}$ frame of reference specifically designed for use with inverter models to evaluate the machine-inverter interaction. Unlike the $\mathrm{d}-\mathrm{q}$ model, the phase variable machine model take into consideration the inductances rotor position dependence, flux linkage rotor position dependence due to permanent magnets, low frequency saturation, eddy currents, cogging torque and geometrical effects such as slotting. Another advantage of developing this model was to enhance the speed of operation. FE model takes a long time to attain a solution while phase variable model reaches steady state within a short time period compared to FE model.

Due to high performance, permanent magnet synchronous machines are becoming quite popular throughout the industry. Therefore in this dissertation these types of machines are considered. System simulators like Simulink provide a user-friendly interface for the dynamic modeling of electrical machines. In Simulink, there are different kinds of forced-commutated electronic switches such as IGBTs, MOSFETs, and 
GTOs which can be used to study an integrated motor drive system [27]. Also different control algorithms and controller design can easily be implemented. Therefore the Simulink is selected as the system simulator and all models have to be Simulink compatible.

\subsubsection{Details of the phase variable model}

This section describes the mathematical foundation for the physical phase variable model. Equations (5-1) through (5-5) describe the system of electrical and mechanical equations governing the machine in the abc frame of reference.

$$
\begin{aligned}
& V_{a b c}=R_{a b c a b c} i_{a b}+d \psi_{a b c}\left(i_{a b c}, \theta\right) / d t \\
& \psi_{a b c}\left(i_{a b c}, \theta\right)=\psi_{s a b c}\left(i_{a b c}, \theta\right)+\psi_{r a b c}(\theta) \\
& T_{m}=p\left(\frac{1}{2} i_{a b c}^{T} \frac{d L_{a b c}\left(i_{a b c}, \theta\right)}{d \theta} i_{a b c}+i_{a b c}^{T} \frac{d \psi_{r a b c}(\theta)}{d \theta}\right)+T_{c o g}(\theta) \\
& J \frac{d \omega}{d t}=T_{m}-B \omega-T_{L} \\
& \frac{d \theta}{d t}=\omega
\end{aligned}
$$

Where,

$V_{a b c}, i_{a b c}:$ terminal voltage vector and current vector

$\theta, \omega$ : rotation angle/rotor position, and angular speed

$R_{a b c}, L_{a b c}\left(i_{a b c}, \theta\right):$ resistance matrix and inductance matrix of stator winding, $\psi_{a b c}\left(i_{a b c}, \theta\right), \psi_{s a b c}\left(i_{a b c}, \theta\right), \psi_{r a b c}(\theta):$ total stator winding flux linkage vector, stator winding flux linkage contributed by its own currents and flux linkage contributed by rotor $\mathrm{PM}$ 
$T_{m}, T_{c o g}(\theta), T_{L}$ : output torque, cogging torque, and load torque respectively

$p \quad$ : number of pole pairs.

$J, B$ : inertia and friction factor

$$
\begin{aligned}
\psi_{s a b c} & =\left[\begin{array}{lll}
\psi_{s a} & \psi_{s b} & \psi_{s c}
\end{array}\right]^{T}=L_{a b c}\left(i_{a b c}, \theta\right) i_{a b c} \\
& =\left[\begin{array}{lll}
L_{a a}\left(i_{a b c}, \theta\right) & L_{a b}\left(i_{a b c}, \theta\right) & L_{a c}\left(i_{a b c}, \theta\right) \\
L_{b a}\left(i_{a b c}, \theta\right) & L_{b b}\left(i_{a b c}, \theta\right) & L_{b c}\left(i_{a b c}, \theta\right) \\
L_{c a}\left(i_{a b c}, \theta\right) & L_{c b}\left(i_{a b c}, \theta\right) & L_{c c}\left(i_{a b c}, \theta\right)
\end{array}\right]\left[\begin{array}{l}
i_{a} \\
i_{b} \\
i_{c}
\end{array}\right]
\end{aligned}
$$

For the PM motor, $L_{a b c}\left(i_{a b c}, \theta\right)$ is current-independent. It can be simplified as $L_{a b c}(\theta)$. $L_{a b c}(\theta)$ is obtained by using the energy perturbation method based on the nonlinear transient FE analysis. The inductances calculated are apparent inductances. $T_{c o g}(\theta)$ as well as $\psi_{r a b c}(\theta)$ are evaluated through a transient nonlinear FE analysis without applying stator winding excitation.

This model is built using SimPowerSystems Simulink toolbox elements e.g. controlled voltage source, resistor etc. as well as user developed components such as adjustable inductance component. For nonlinear saturated inductances, there are three kinds of definitions: apparent inductance $L_{a p p}$, effective inductance $L_{\text {eff }}$ and incremental inductance $L_{\text {inc }}$.

$$
\begin{aligned}
& L^{a p p}=\psi / i \\
& L^{e f f}=2 W / i^{2} \\
& L^{i n c}=\partial \psi / \partial i
\end{aligned}
$$

Taking the derivative of Eq. (5-2) w.r.t. time yields: 
$\left[\begin{array}{l}d \psi_{a} / d t \\ d \psi_{b} / d t \\ d \psi_{c} / d t\end{array}\right]=\left[\begin{array}{lll}\partial \psi_{s a} / \partial i_{a} & \partial \psi_{s a} / \partial i_{b} & \partial \psi_{s a} / \partial i_{c} \\ \partial \psi_{s b} / \partial i_{a} & \partial \psi_{s b} / \partial i_{b} & \partial \psi_{s b} / \partial i_{c} \\ \partial \psi_{s c} / \partial i_{a} & \partial \psi_{s c} / \partial i_{b} & \partial \psi_{s c} / \partial i_{c}\end{array}\right]\left[\begin{array}{l}\partial i_{a} / \partial t \\ \partial i_{b} / \partial t \\ \partial i_{c} / \partial t\end{array}\right]+\left[\begin{array}{l}\partial \psi_{s a} / \partial \theta \\ \partial \psi_{s b} / \partial \theta \\ \partial \psi_{s c} / \partial \theta\end{array}\right] \frac{\partial \theta}{\partial t}+\left[\begin{array}{l}d \psi_{r a} / d t \\ d \psi_{r b} / d t \\ d \psi_{r c} / d t\end{array}\right]$

Where, $\psi_{s a}\left(i_{a b c}, \theta\right), \psi_{s b}\left(i_{a b c}, \theta\right)$ and $\psi_{s c}\left(i_{a b c}, \theta\right)$ are flux linkages contributed by the winding currents. They can be expressed using the apparent inductances as follows:

$$
\begin{aligned}
& \psi_{s a}\left(i_{a b c}, \theta\right)=L_{a a}^{a p p}\left(i_{a b c}, \theta\right) i_{a}+L_{a b}^{a p p}\left(i_{a b c}, \theta\right) i_{b}+L_{a c}^{a p p}\left(i_{a b c}, \theta\right) i_{c} \\
& \psi_{s b}\left(i_{a b c}, \theta\right)=L_{b a}^{a p p}\left(i_{a b c}, \theta\right) i_{a}+L_{b b}^{a p p}\left(i_{a b c}, \theta\right) i_{b}+L_{b c}^{a p p}\left(i_{a b c}, \theta\right) i_{c} \\
& \psi_{s c}\left(i_{a b c}, \theta\right)=L_{c a}^{a p p}\left(i_{a b c}, \theta\right) i_{a}+L_{c b}^{a p p}\left(i_{a b c}, \theta\right) i_{b}+L_{c c}^{a p p}\left(i_{a b c}, \theta\right) i_{c}
\end{aligned}
$$

Considering that the inductance of a PM motor is independent of the stator winding current, one can obtain from (5-10), (5-11), (5-12) and (5-13):

$$
\begin{aligned}
{\left[\begin{array}{l}
d \psi_{a} / d t \\
d \psi_{b} / d t \\
d \psi_{c} / d t
\end{array}\right]=} & {\left[\begin{array}{lll}
L_{a a}^{a p p}(\theta) & L_{a b}^{a p p}(\theta) & L_{a c}^{a p p}(\theta) \\
L_{b a}^{a p p}(\theta) & L_{b b}^{a p p}(\theta) & L_{b c}^{a p p}(\theta) \\
L_{c a}^{a p p}(\theta) & L_{c b}^{a p p}(\theta) & L_{c c}^{a p p}(\theta)
\end{array}\right]\left[\begin{array}{l}
d i_{a} / d t \\
d i_{b} / d t \\
d i_{c} / d t
\end{array}\right] } \\
& +\left[\begin{array}{lll}
d L_{a a}^{a p p}(\theta) / d \theta & d L_{a a}^{a p p}(\theta) / d \theta & d L_{a a}^{a p p}(\theta) / d \theta \\
d L_{a a}^{a p p}(\theta) / d \theta & d L_{a a}^{a p p}(\theta) / d \theta & d L_{a a}^{a p p}(\theta) / d \theta \\
d L_{a a}^{a p p}(\theta) / d \theta & d L_{a a}^{a p p}(\theta) / d \theta & d L_{a a}^{a p p}(\theta) / d \theta
\end{array}\right]\left[\begin{array}{l}
i_{a} \\
i_{b} \\
i_{c}
\end{array}\right] \omega+\left[\begin{array}{c}
e_{a}(\theta) \\
e_{b}(\theta) \\
e_{c}(\theta)
\end{array}\right]
\end{aligned}
$$

Dropping the apparent notation and substituting (5-14) in (5-1), we obtain voltage equation as follows:

$$
\begin{aligned}
& {\left[\begin{array}{l}
v_{a} \\
v_{b} \\
v_{c}
\end{array}\right]=\left[\begin{array}{lll}
r_{a} & 0 & 0 \\
0 & r_{b} & 0 \\
0 & 0 & r_{c}
\end{array}\right]\left[\begin{array}{l}
i_{a} \\
i_{b} \\
i_{c}
\end{array}\right]+\left[\begin{array}{lll}
L_{a a} & L_{a b} & L_{a c} \\
L_{b a} & L_{b b} & L_{b c} \\
L_{c a} & L_{c b} & L_{c c}
\end{array}\right]\left[\begin{array}{l}
d i_{a} / d t \\
d i_{b} / d t \\
d i_{c} / d t
\end{array}\right]} \\
& +\left[\begin{array}{lll}
d L_{a a} / d \theta & d L_{a b} / d \theta & d L_{a c} / d \theta \\
d L_{b a} / d \theta & d L_{b b} / d \theta & d L_{b c} / d \theta \\
d L_{c a} / d \theta & d L_{c b} / d \theta & d L_{c c} / d \theta
\end{array}\right]\left[\begin{array}{c}
i_{a} \\
i_{b} \\
i_{c}
\end{array}\right] \omega+\left[\begin{array}{c}
e_{a} \\
e_{b} \\
e_{c}
\end{array}\right]
\end{aligned}
$$

For clarity, writing above equation individually. 


$$
\begin{aligned}
& v_{a}=i_{a} r_{a}+\left[L_{a a}(\theta) \frac{d i_{a}}{d t}+L_{a b}(\theta) \frac{d i_{b}}{d t}+L_{a c}(\theta) \frac{d i_{c}}{d t}\right]+\left[\frac{\partial \psi_{a L}(\theta)}{\partial \theta} \omega+e_{a}(\theta)\right] \\
& v_{b}=i_{b} r_{b}+\left[L_{b a}(\theta) \frac{d i_{a}}{d t}+L_{b b}(\theta) \frac{d i_{b}}{d t}+L_{b c}(\theta) \frac{d i_{c}}{d t}\right]+\left[\frac{\partial \psi_{b L}(\theta)}{\partial \theta} \omega+e_{b}(\theta)\right] \\
& v_{c}=i_{c} r_{c}+\left[L_{c a}(\theta) \frac{d i_{a}}{d t}+L_{c b}(\theta) \frac{d i_{b}}{d t}+L_{c c}(\theta) \frac{d i_{c}}{d t}\right]+\left[\frac{\partial \psi_{c L}(\theta)}{\partial \theta} \omega+e_{c}(\theta)\right]
\end{aligned}
$$

The first two terms of (5-15) are the Faraday voltage drop due to the time variation of the flux linkage caused by the machine windings' currents. Concretely, the first term is the standard transformer voltage drop. The second term is rotational voltage drop. The third term $e_{a}(\theta), e_{b}(\theta)$ and $e_{c}(\theta)$ are the back EMF contributed by the permanent magnet.

The apparent inductance terms are calculated by using energy perturbation method with the transient FE solutions of the motor magnetic fields.

\subsubsection{Simulink implementation}

This section presents the procedure to develop the circuit element based model. Equations (5-16)-(5-18) give the circuit model description of the PM motor, which is shown in Fig. 5.1. Standard resistor component and controlled voltage source component in SimPowerSystems Simulink toolbox are used to represent the winding resistance and the voltage drop due to rotor rotation and the magnetic field generated by permanent magnet. As inductances are the function of rotor position, adjustable inductance component is developed. There are two ways to describe inductances:

$$
\begin{aligned}
& v_{L}=L * d i_{L} / d t \\
& i_{L}=i_{L}(0)+\frac{1}{L} \int_{0}^{i} V_{L} d t
\end{aligned}
$$




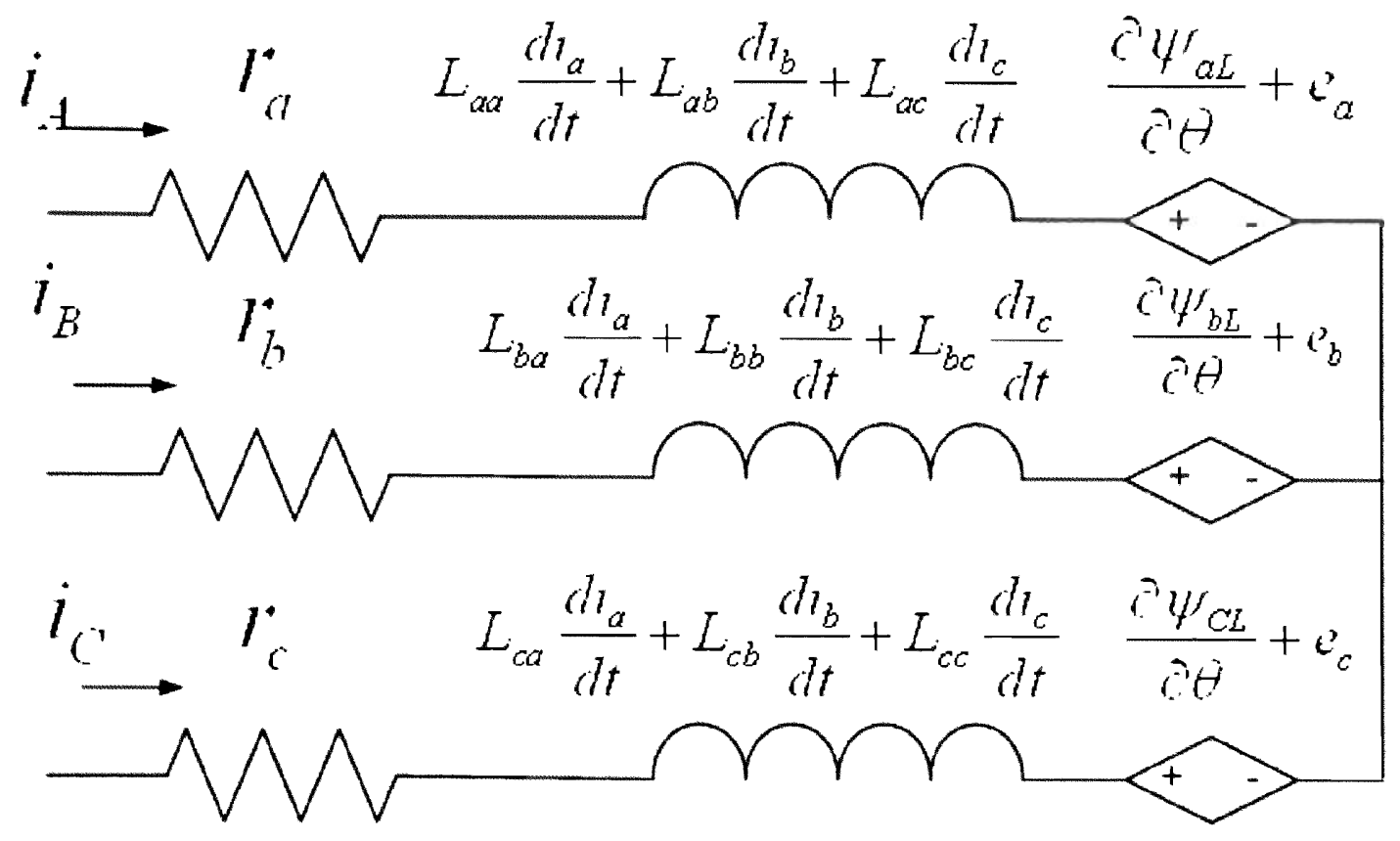

Fig.5.1. Circuit element based representation of stator winding

As the inductance current is a state variable, the inductance block should contain a way to identify the initial current value. Since the derivative expression given by equation (5-19), can't meet this requirement. Therefore, we adopt the integral description in (5-20) for the construction of the adjustable inductance block. The implementation in Simulink is shown in Fig. 5.2. The initial value of the integrator needs to be set to zero. The voltage measurement block (VM) and the controlled current source (CCS) make it possible for the adjustable inductance block to be connected to other circuit components available in Simulink. In other words, this converts the mathematical model (3-18) into an electrical component. Then, one can build the inductance components of each phase. As an example, Fig. 5.3 gives the inductance block of phase a, expressed mathematically as follows: 


$$
V_{l a}=L_{a a} \frac{d i_{a}}{d t}+L_{a b} \frac{d i_{b}}{d t}+L_{a c} \frac{d i_{c}}{d t}
$$

The upper left part of Fig. 5.3 is the diagram shown in Fig. 5.2. It gives phase a self inductance voltage drop. The mutual-inductance voltage drop is represented by two controlled voltage sources (CVS). The current passing through the weighted line is $i_{a}$. The self-inductance $L_{a a}$, mutual inductances $L_{a b}$ and $L_{a c}$ are retrieved from the inductance database by picking up inductance values corresponding to a specific rotor position. The controlled voltage source block is shown in Fig. 5.4. The current passing through the "in" and "out" ports is the phase current $i_{a}$.

\subsubsection{Case studies}

In order to evaluate the developed model performance, several case studies were carried out. The PM motor is fed by a current-controlled PWM inverter which is built using a Universal Bridge block.

Figure 5.5 shows the speed, currents, torque, and the back emf simulation results for motor starting at full voltage.

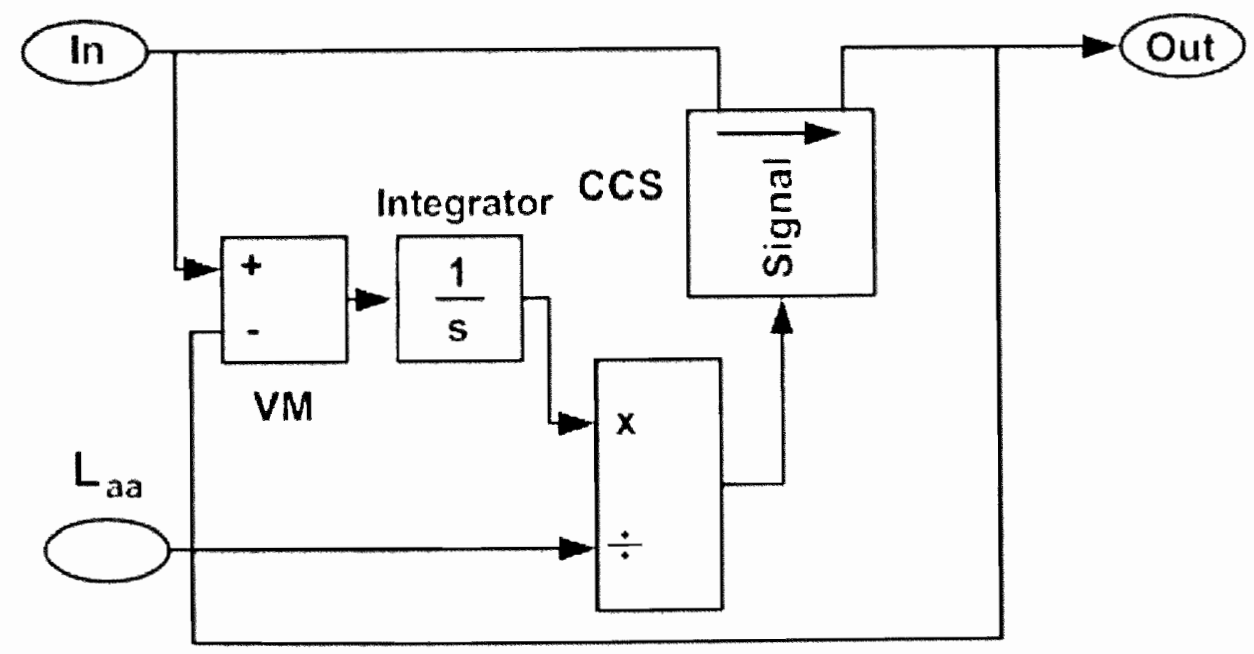

Fig.5.2. Adjustable self- inductance 


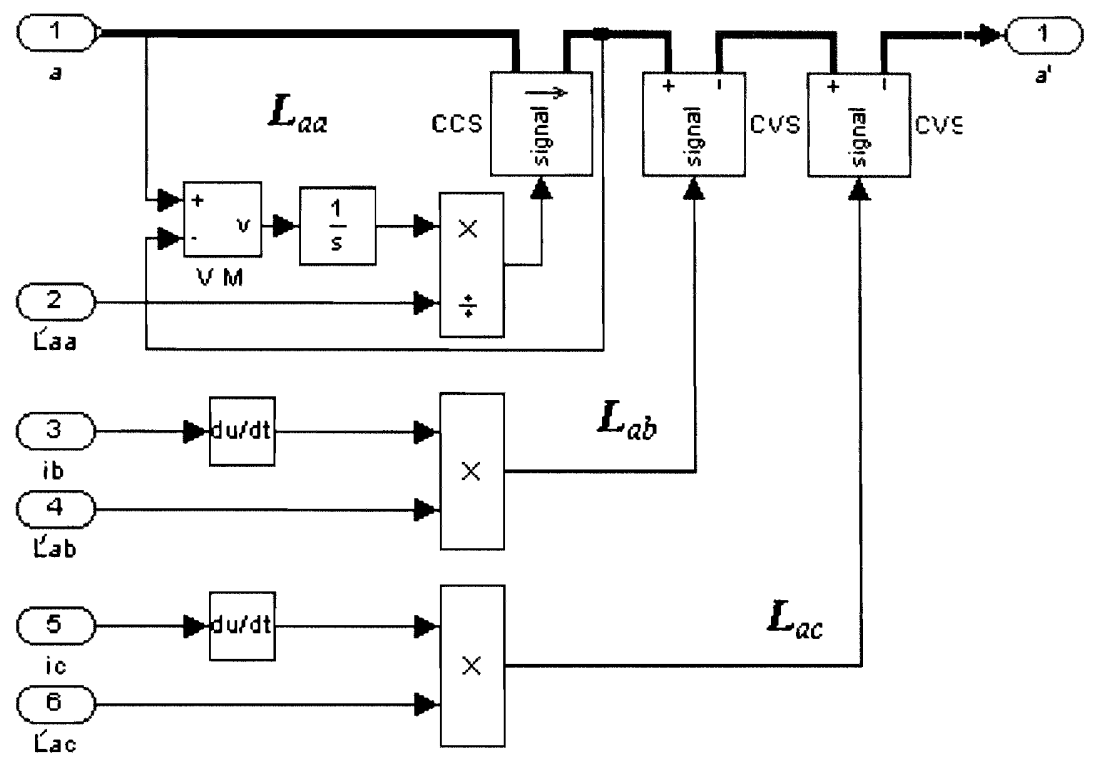

Fig.5.3. Inductance block of phase A

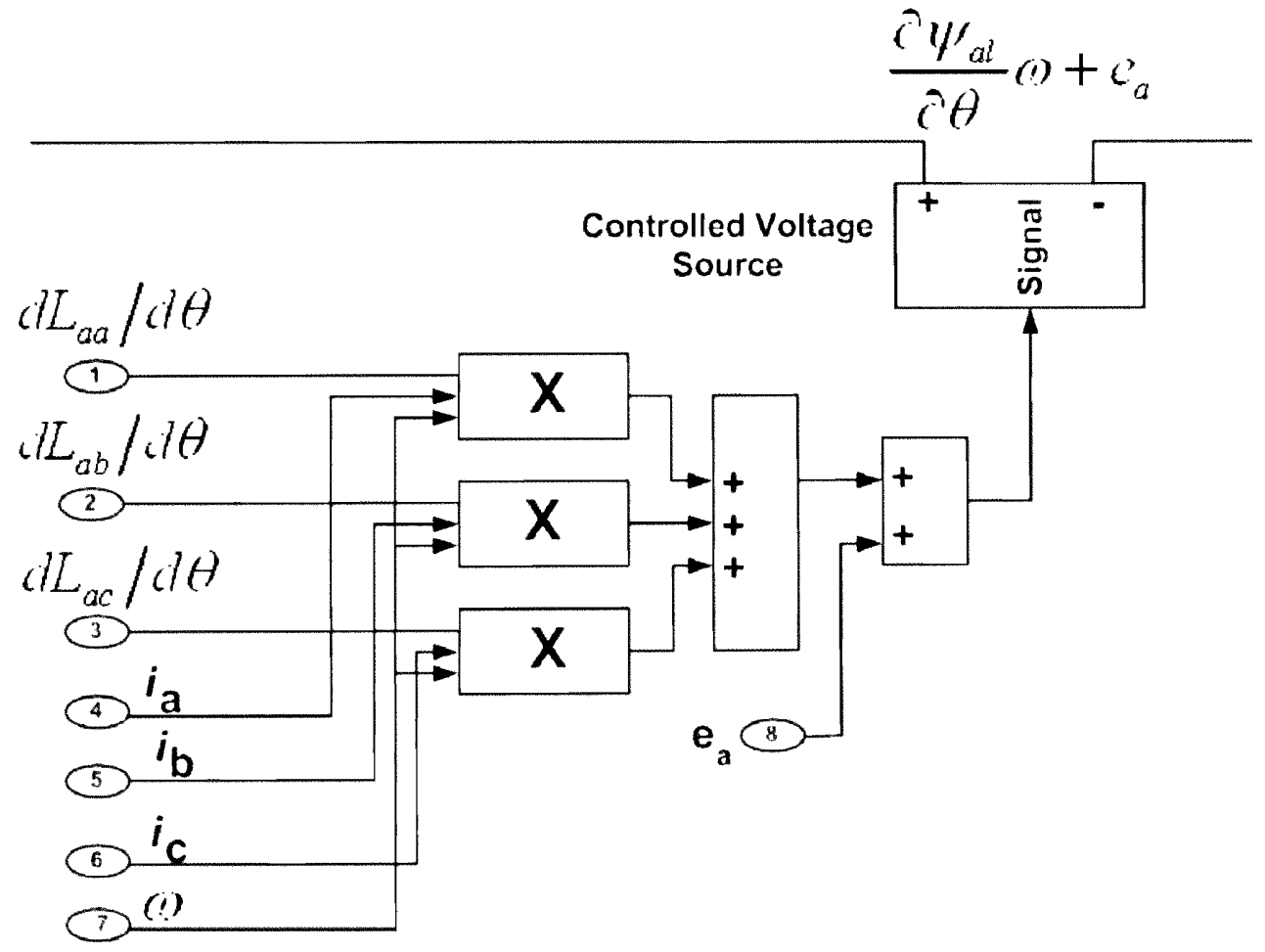

Fig.5.4. Controlled voltage source 


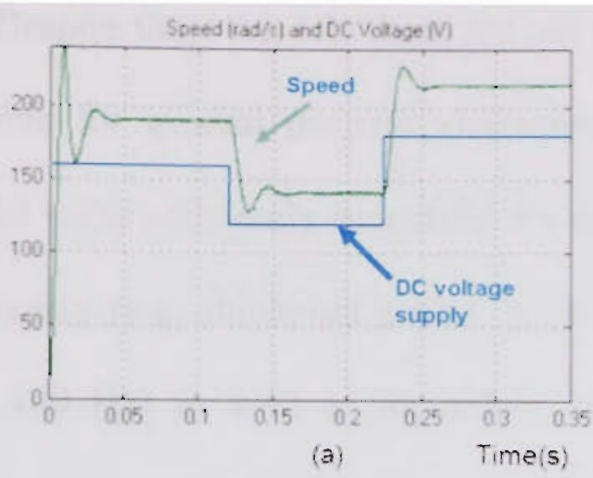

Toroue $\mathrm{Nm}$

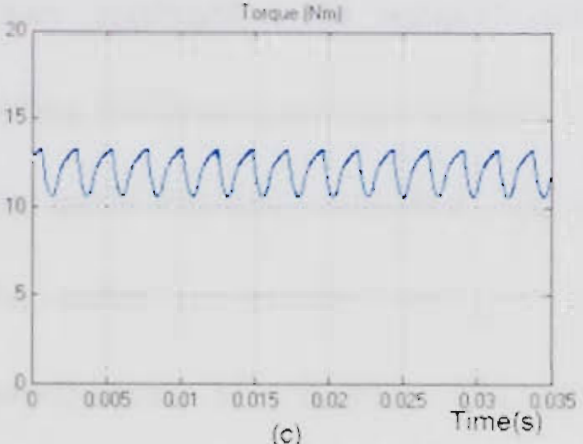

(c)

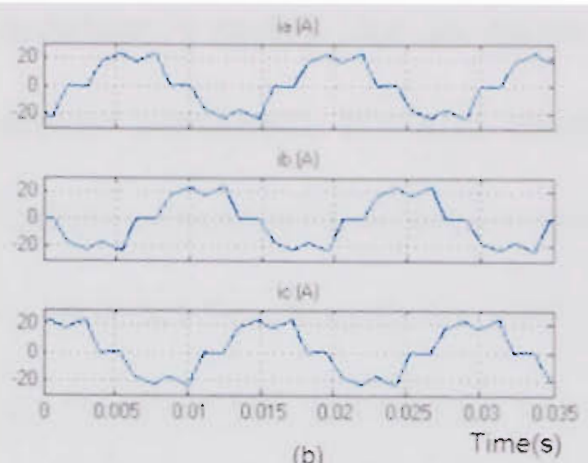

(b)

Bask E.NF M

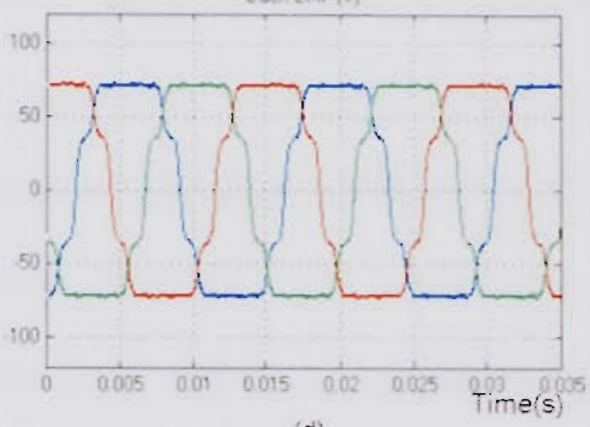

(d)

Fig.5.5. The drive system simulation results

\subsection{Cable modeling}

\subsubsection{The model}

The transient behavior of power cables is difficult to model accurately, because of the strong dependence of the cable parameters on the power system operating conditions. A number of well-established models can be found in the literature $[128,136]$.

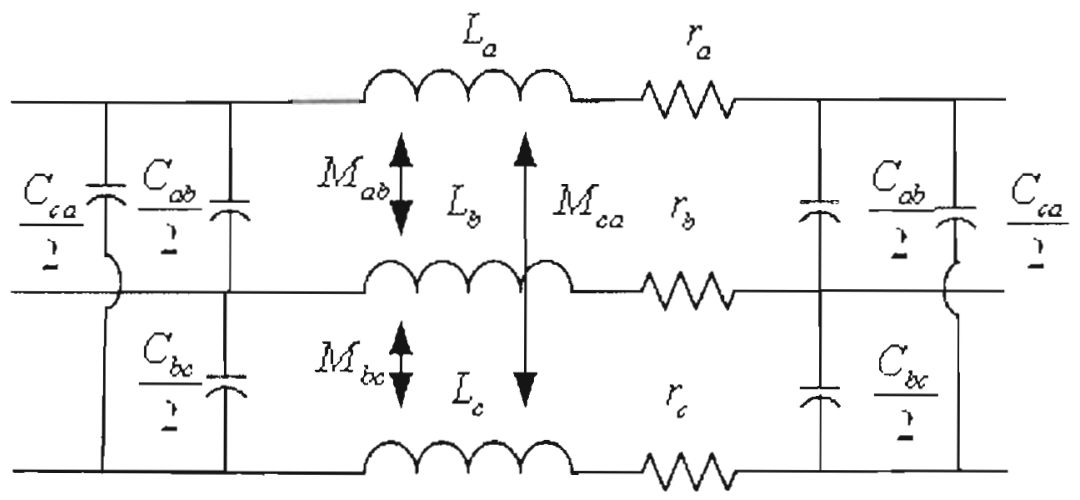

Fig.5.6. The lumped cable model 
Despite these models there are not yet fully satisfactory models that are simple and reliable for general purpose electromagnetic transient simulation. It's also crucial to model cable efficiently especially for shipboard energy systems with different operating frequency (e.g. shipboard power system in which there are two frequencies used $60 \mathrm{~Hz}$ and $400 \mathrm{~Hz}$ ) as well as the effect of harmonics on the cable parameters. For Low frequency application the lumped cable model is used. The cable is modeled by connecting different sections together in series, the number of sections depend on the required cable frequency response. The cable model per unit length is shown in Fig. 5.6

The cable parameters per unit length were obtained from electrostatic and magnetodynamic FE analysis. This included the cable resistance, capacitances, and inductances.

These cable parameters obtained from FE were used to build an abc frame cable Simulink model. The abc model represent the electrically separate but magnetic coupled three phase conductors of the cable by a set of first order linear differential equations. The model differential equations are as follows:

$$
\begin{aligned}
& V_{1}=R_{1} i_{1}+L_{1} \frac{d i_{1}}{d t}+M_{12} \frac{d i_{2}}{d t}+M_{13} \frac{d i_{3}}{d t} \\
& V_{2}=R_{2} i_{2}+L_{2} \frac{d i_{2}}{d t}+M_{21} \frac{d i_{1}}{d t}+M_{23} \frac{d i_{3}}{d t} \\
& V_{3}=R_{3} i_{3}+L_{3} \frac{d i_{3}}{d t}+M_{31} \frac{d i_{1}}{d t}+M_{32} \frac{d i_{2}}{d t}
\end{aligned}
$$

The cable phase to ground and phase to phase capacitances are divided equally on both sides of the cable. This is known as the PI-model.

The significance of using FEM to extract the cable parameters were shown to be accurate and eliminates costly cable testing. 


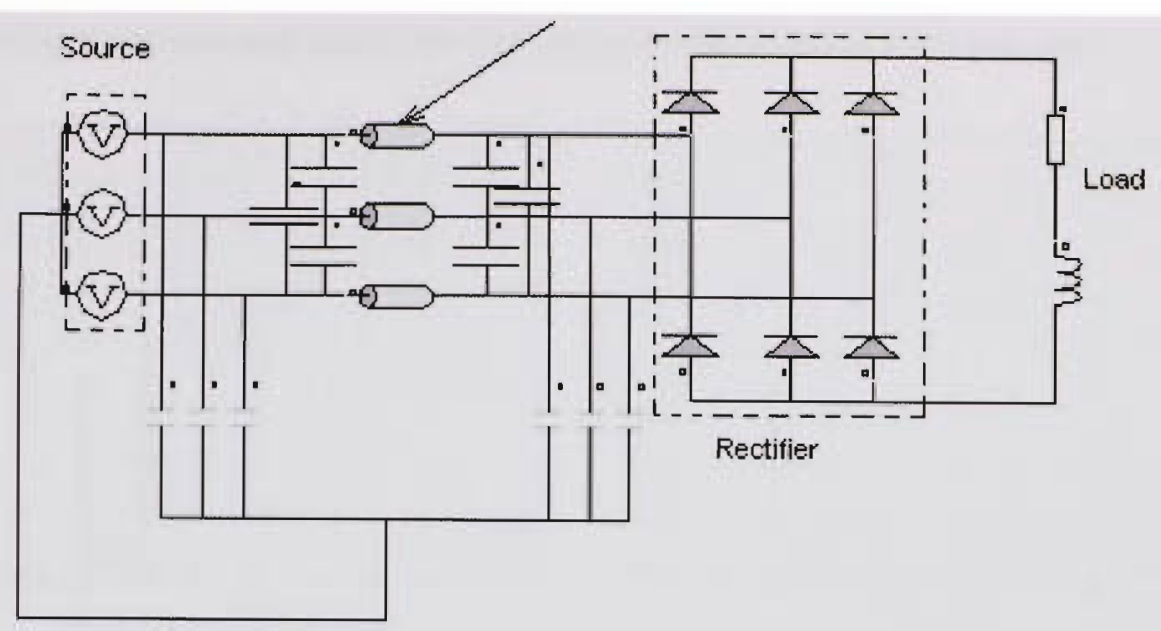

Fig.5.7. the System used in the study

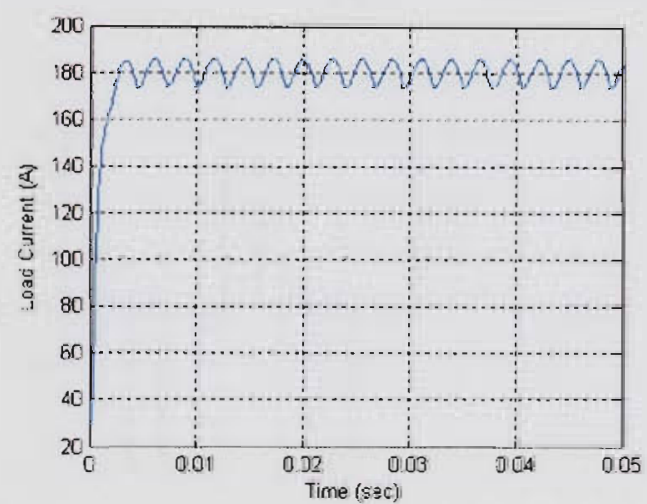

(a) From abc model

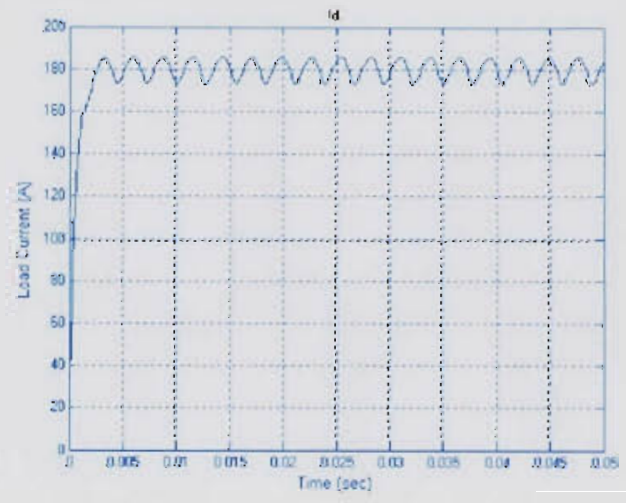

(b) From FE

Fig.5.8. The DC side current

\subsubsection{Simulation results}

A $208 \mathrm{~V}, 60 \mathrm{~Hz}$ three phase supply is used to energize the cable. The rectifier load has $\mathrm{L}=1.5 \mathrm{mH}$, and $\mathrm{R}=2.5$. Figure $5-7$ shows the system used in the study.

Figure 5-8 through Fig. 5-10 shows, respectively the waveform related to the load current, cable phase a current, and voltage drop across the cable obtained from the abc model and FEM model respectively. The voltage drop waveform is substantially distorted by the nonlinear load. 
The voltage notches and nicks are due to the diode commutation process.

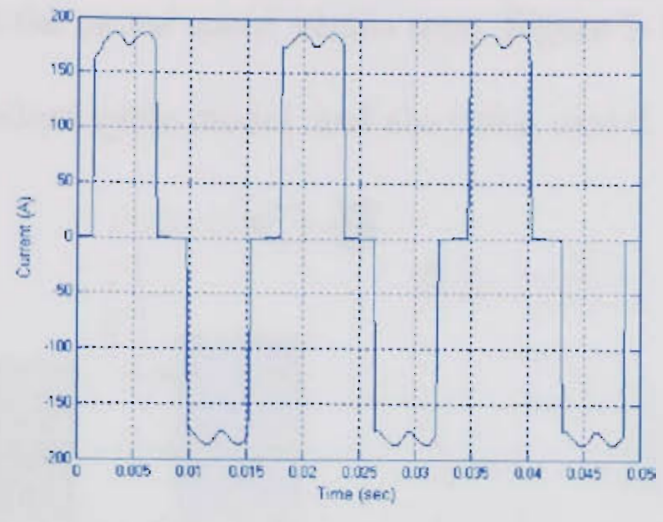

(a) From abc model

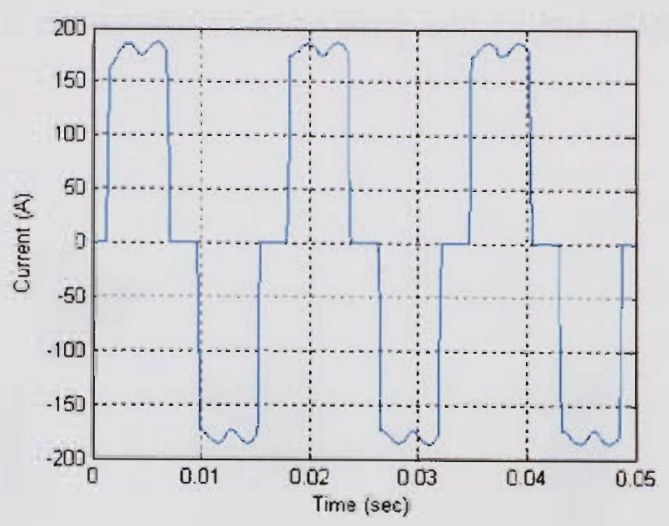

(b) From FE

Fig.5.9 Cable phase a current waveform

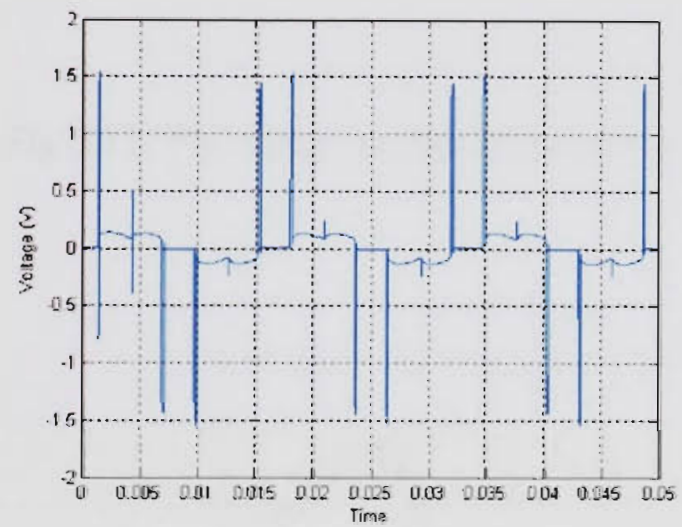

(a) From abc model

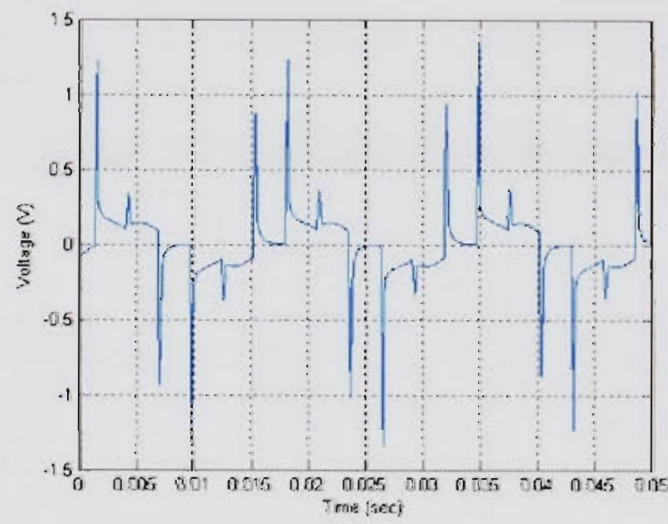

(b) From FE

Fig.5.10. The voltage drop across the cable

It clear that the terminal behavior of the cable and the abc model is the same, except a small difference in the voltage waveform obtained from abc model.

The second case study was performed by connecting the developed PM motor model to a voltage source through the cable developed. The overall system Simulink model is shown in Fig. 5.11. The system consists of the source, the cable model, rectifier, inverter, the PM motor phase variable model, and the speed controller. 
Figure 5-12 through 5-14 shows the motor three phase currents, the motor torque, and the motor speed versus time. Figure 5-15 shows the voltage drop across the cable for standard cable model, and abc cable model.

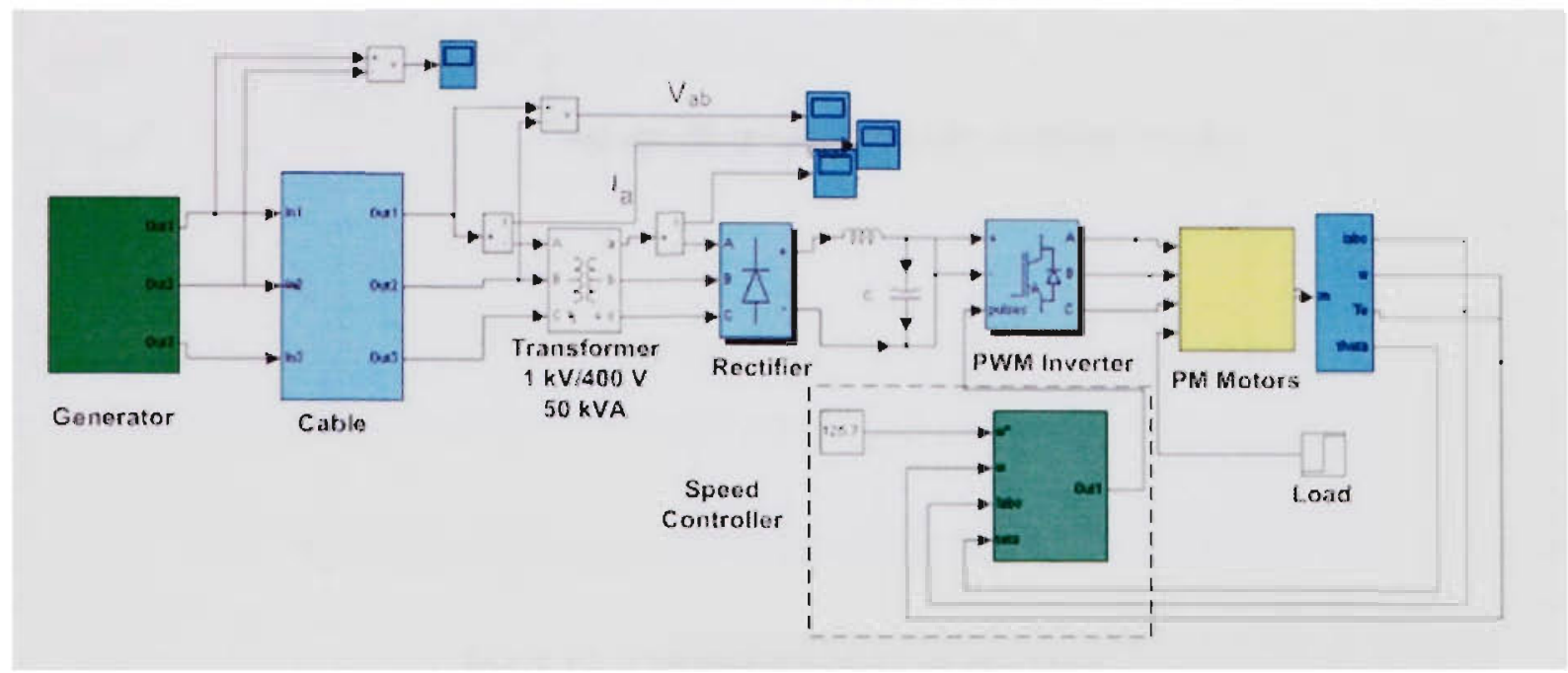

Fig.5.11. The motor model connected to a voltage source through the cable model

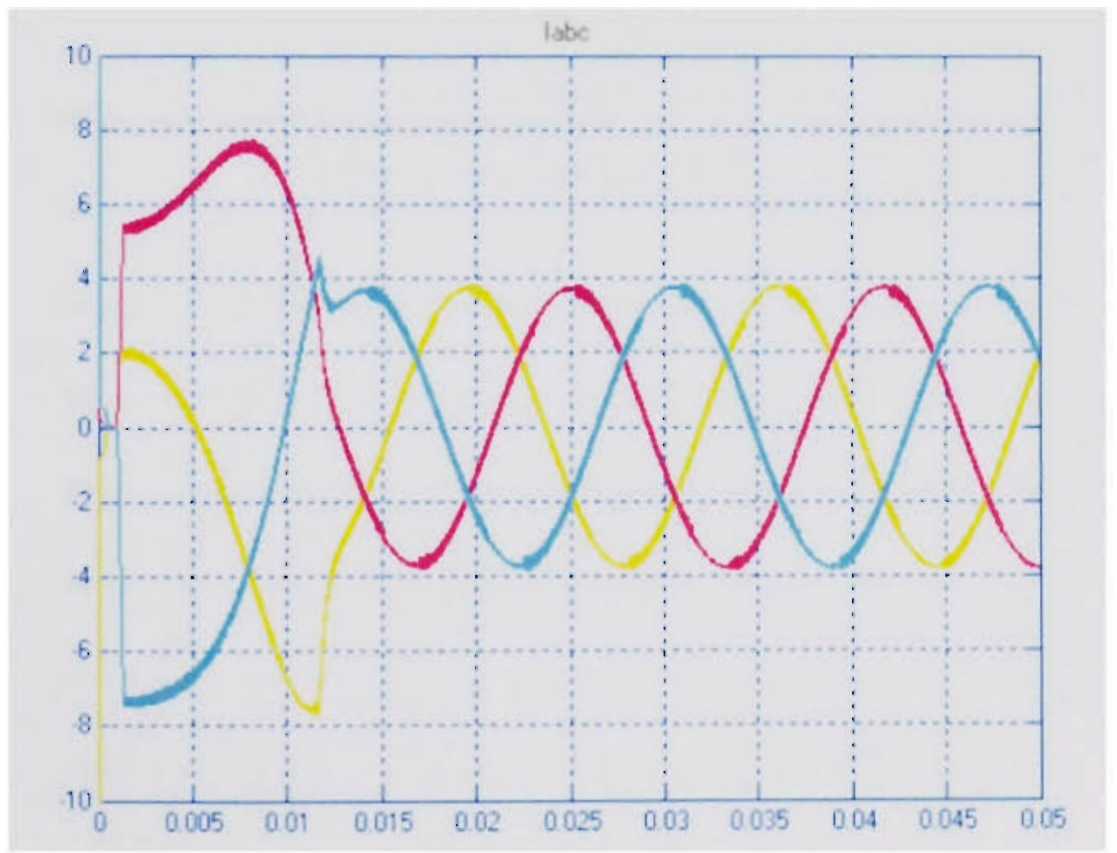

Fig.5.12. The motor three phase currents 


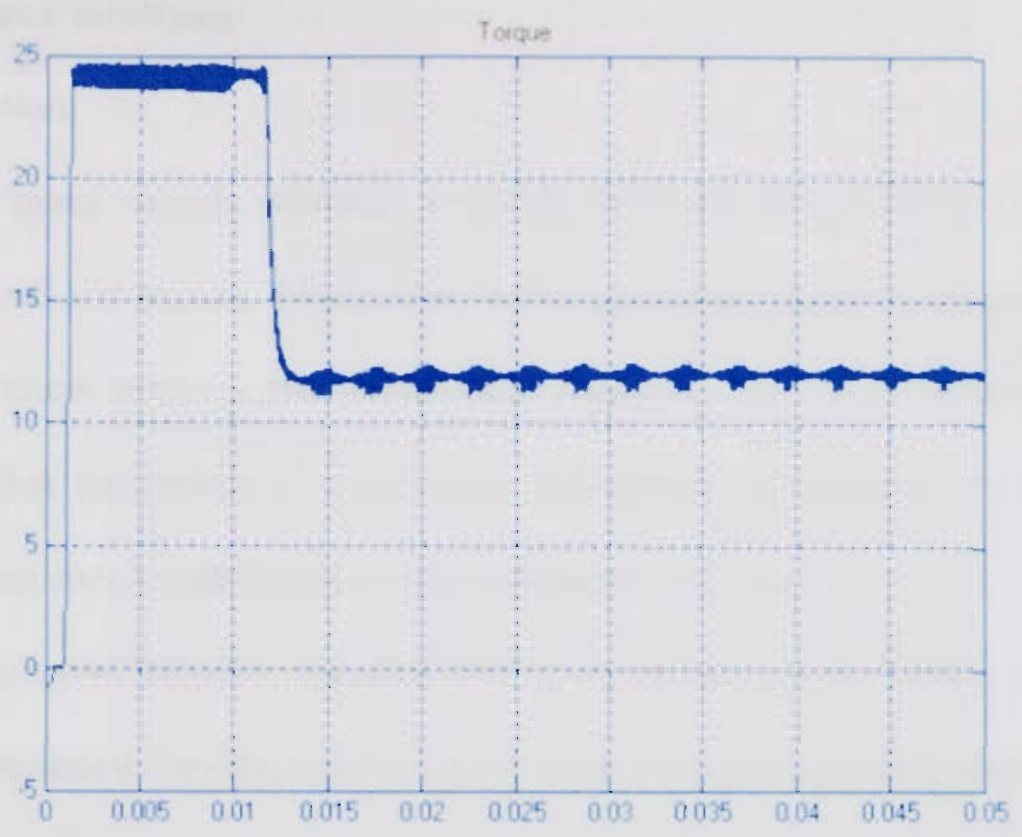

Fig.5.13. The motor torque versus time

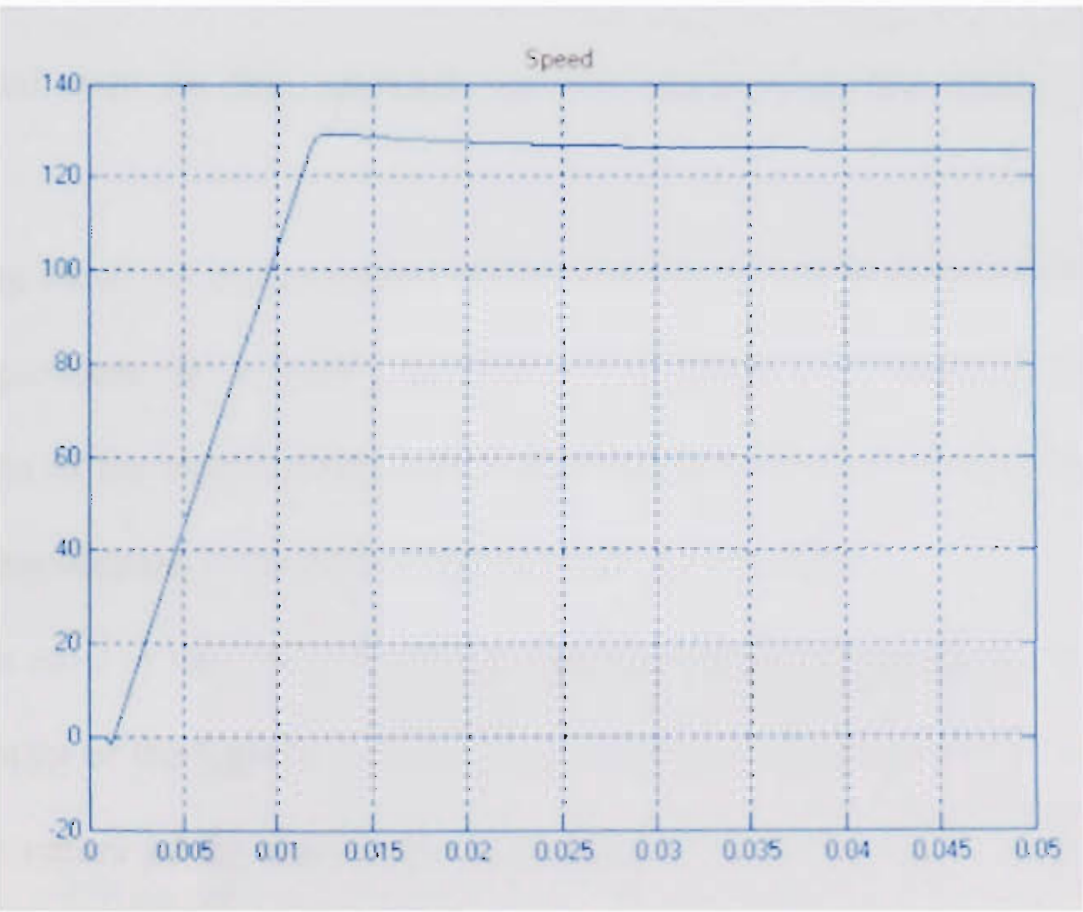

Fig.5.14. The motor speed versus time 


\subsection{Transformer modeling}

\subsubsection{Introduction}

There are many models currently available for power transformers [39]. Modeling based on the self and mutual inductances; which utilize formulae to calculate of self and mutual inductances between the transformer windings. The main disadvantage of this technique is that the values of transformer inductances are close in value due to the transformer iron core which results in ill-conditioned equations.

Geomagnetically induced currents (GIC) saturation model which use nonlinear resistance to represent the effect of the saturation in the equivalent magnetic circuit.

Modeling based on leakage inductance which represents adequately the leakage inductances (i.e. load or short circuit conditions) of a transformer but iron core is not properly represented.

Modeling based on principle of duality which deals accurately with the iron core, but models based only on this approach are not representing the leakage inductances correctly.

Modeling based on measurements which focuses on representing a transformer as a terminal equivalent in a wide spectrum of frequencies. Tests are made for the determination of the model parameters in the frequency domain or time domain. Weak basis for generalization is the major drawback of those methods.

However most of these models assume that the transformer parameters do not change with the change of the transformer operating condition. Most of these models ignore the time-variant nature of the transformer inductances and their effect on the transformer operation. 
The time variant nature of the transformer inductance was demonstrated in chapter 3 . This will make most of these models inaccurate especially when predicating the transformer harmonic behavior under non-linear load.

\subsubsection{Transformer phase variable model}

The main disadvantage of the FEM model is the excessive longer simulation time and high processing power requirement. This in turn will increase the overall system simulation time. Using the FE model for the transformer takes into consideration details that cannot be included by currently available methods (e.g. inductance time variant, effect of core saturation, and skin effects).

Phase variable will provide us with the ability to integrate the transformer model within different types of studies while maintaining short simulation time. The model parameters are derived from the from nonlinear transient $\mathrm{FE}$ solutions for the transformer.

The three phase transformer is modeled as a three two winding transformer. The self and mutual inductances between the different primary and secondary coils obtained in chapter 3 were utilized. The two winding transformer voltage, flux linkage, currents, and mutual flux linkage are described by the following mathematical equations [40]:

$$
\begin{aligned}
& V_{1}=R_{1} i_{1}+\frac{d \lambda_{1}}{d t} \\
& V_{2}^{\prime}=R_{2} i_{2}^{\prime}+\frac{d \lambda_{2}^{\prime}}{d t} \\
& \lambda_{1}=L_{l 1} i_{1}+\lambda_{m}, \lambda_{2}^{\prime}=L_{l 2}^{\prime} i_{2}^{\prime}+\lambda_{m}, \lambda_{m}=M\left(i_{1}+i_{2}^{\prime}\right) \\
& \lambda_{1}=\int\left\{v_{1}-r_{1}\left(\frac{\lambda_{1}-\lambda_{m}}{L_{l 1}}\right)\right\} d t \\
& \lambda_{2}^{\prime}=\int\left\{v_{2}^{\prime}-r_{2}^{\prime}\left(\frac{\lambda_{2}^{\prime}-\lambda_{m}}{L_{l 2}^{\prime}}\right)\right\} d t
\end{aligned}
$$


$i_{1}=\frac{\lambda_{1}-\lambda_{m}}{L_{l 1}}$ and $i_{2}^{\prime}=\frac{\lambda_{2}^{\prime}-\lambda_{m}}{L_{l 2}^{\prime}}$

$\lambda_{m}=l_{M}\left(\frac{\lambda_{1}}{L_{l 1}}+\frac{\lambda_{2}^{\prime}}{L_{l 2}^{\prime}}\right)$

$\frac{1}{l_{M}}=\frac{1}{M}+\frac{1}{L_{l 1}}+\frac{1}{L_{l 2}^{\prime}}$

Where:

$v_{1}, v_{2}^{\prime}:$ primary side voltage and secondary side voltage referred to primary side

$i_{1}, i_{2}^{\prime}:$ primary side current, and secondary side current referred to primary side

$\lambda_{1}, \lambda_{2}^{\prime}, \lambda_{m}$ : flux linkage of primary side, flux linkage of secondary side referred to primary side, and the mutual flux

$L_{l 1}\left(t, \lambda_{m}\right), L_{l 2}^{\prime}\left(t, \lambda_{m}\right), M\left(t, \lambda_{m}\right):$ The primary leakage inductance, secondary leakage inductance referred to primary side, and the mutual inductance between the primary and secondary as a function of the time, and magnetizing flux.

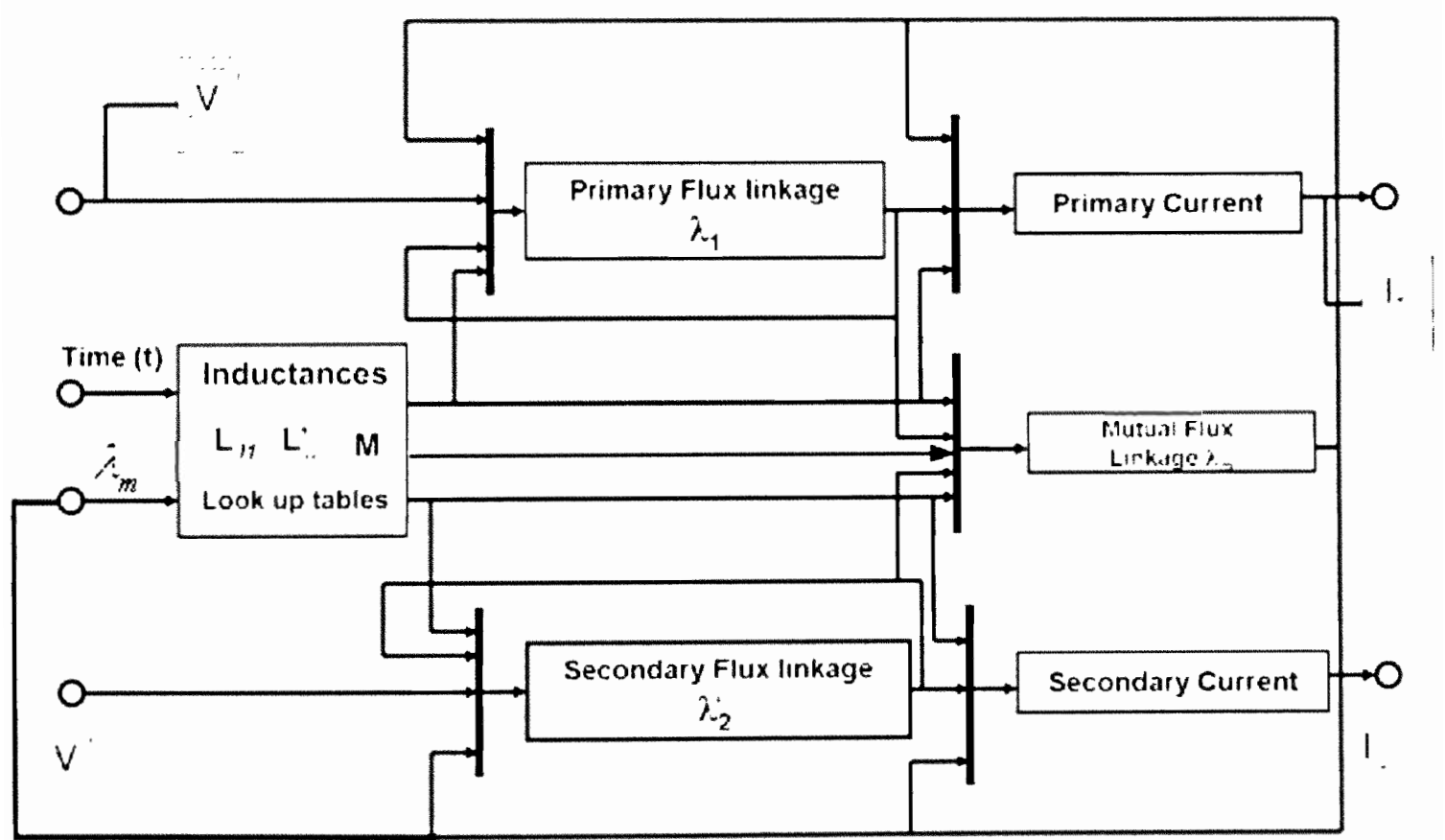

Fig.5.15. Simulink block flow implementation of the transformer model 
The Phase variable model was implemented in Simulink. Figure 5.15 shows the block diagram flow of the transformer phase variable model. The model is essentially a detailed inductance model. The effect of saturation on the transformer leakage inductances is considered by utilizing lookup table for the primary and secondary inductances. The values of lookup table inductances were obtained from nonlinear FE solution for different magnetizing current levels as described before in chapter 3 .

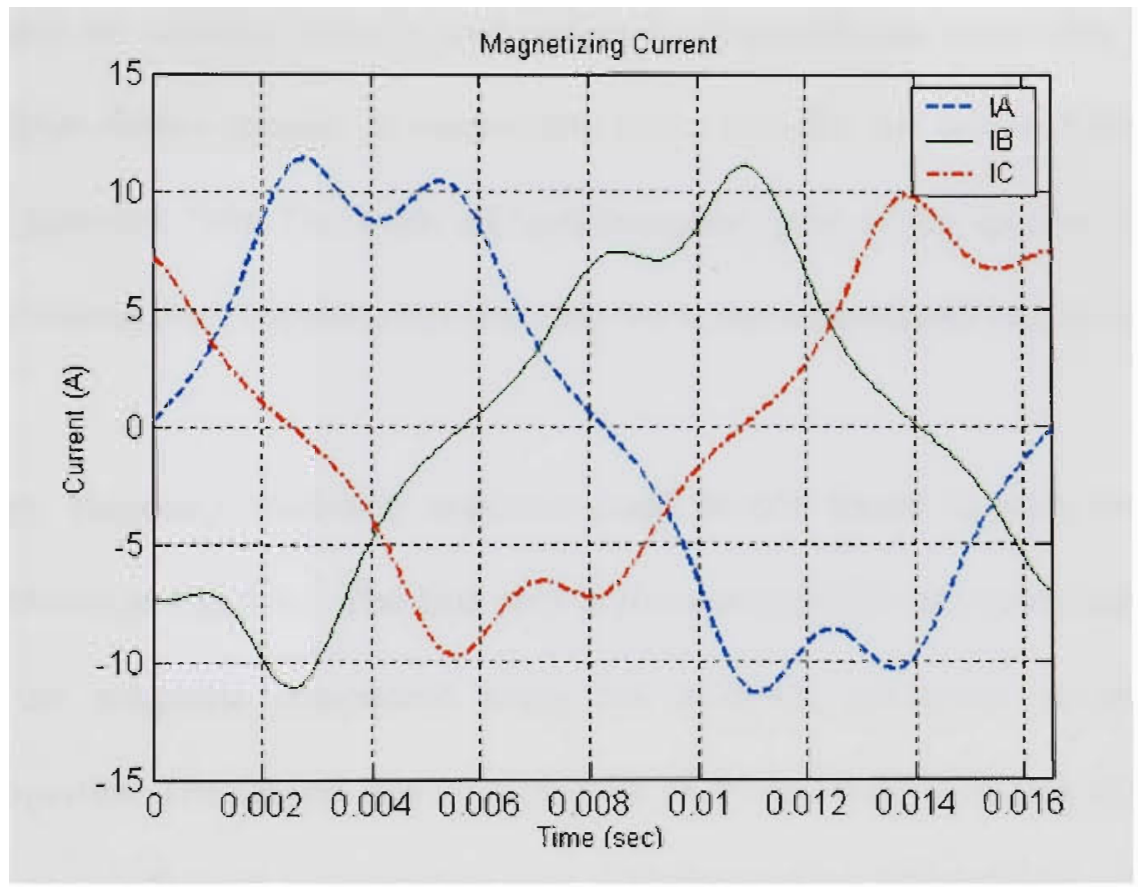

Fig.5.16. Transformer three phase magnetizing current

\subsubsection{Simulations}

Several simulations were conducted to evaluate the developed model response. An open circuit simulation test was conducted by connecting a high resistance on the transformer secondary side. Figure 5.17 shows the transformer three phase magnetizing current. 


\section{Chapter 6: Model Development for Shipboard Power system Components for High Frequency Studies}

\subsection{Introduction}

This chapter deals with high frequency modeling of different shipboard Power system components. High-frequency modeling is essential in the design stage of naval power system components to study the impulse voltage and switching surge distribution. It is also necessary for winding integrity and insulation diagnosis and most often frequency effects for high-fidelity models in bandwidths up to $10 \mathrm{MHz}$ are required for condition monitoring purposes [39]. The study of high-frequency part of the spectra is necessary due to the resulting stray capacitances shunting the series inductances and dominating the response.

The high frequency modeling approach used in this thesis is represented in the Flowchart shown in Fig 6.1. The first step is the construct the geometry that describes physically the magnetic component using the available geometric component and material properties. The second step is to perform FEM simulations. In this step two type of analysis are performed magnetodynamic and electrostatic FE analysis. In the first analysis, a voltage source is applied to the windings and the magnetic fields and current densities in the magnetic component are then calculated in a frequency range. The lower frequency limit of the FE analysis is the equipment normal operating frequency and the upper frequency limit is several $\mathrm{MHz}$, to take into account the frequency related effects. The second analysis is performed in DC conditions. A voltage is applied to the conductors and the electric fields and stored energy in the components is then calculated. 
The third step is to use the result of FE simulations in step 2 to calculate the model parameters as descried in chapter 2 .

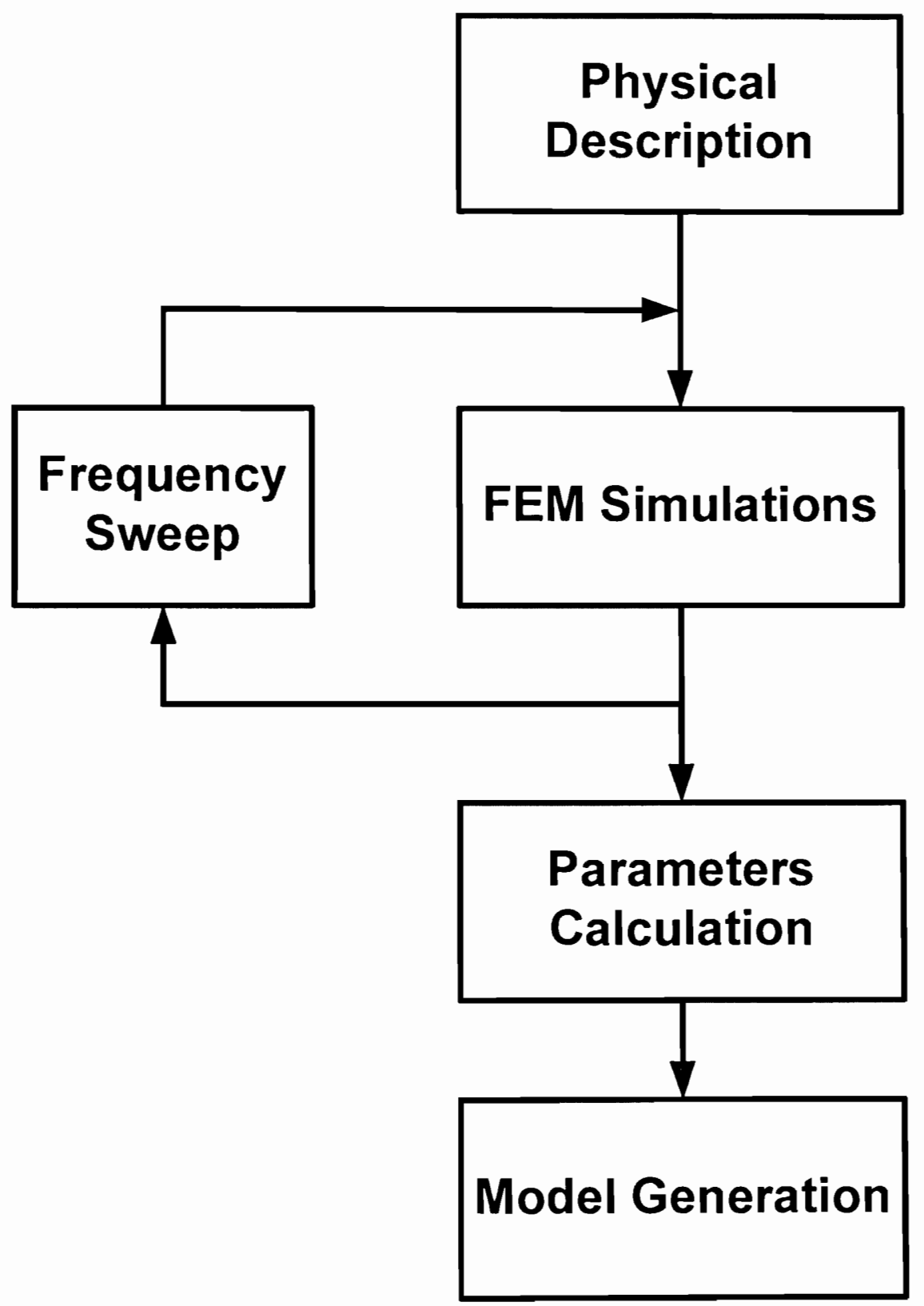

Fig.6.1. Flowchart of HF model generation procedure

Self capacitances of each conductor as well as the mutual capacitances between turns were calculated by electrostatic FE analysis. The fourth step is to build a model that can 
be used in the chosen system simulator (e.g. Simulink). This technique will allow the physical representation of the nonlinear magnetic core behavior of the component as well as the strong frequency dependence of the component parameters.

A turn-to-turn based model would be unnecessary complex and require excessive computational capabilities. When lumping several turns to one electrical lumped element, the order of the model is reduced. This is done by shifting the capacitances connected to internal coil nodes to the external ones.

Another approach is to consider the frequency dependence of the magnetic comments behavior on the source frequency. The Frequency response is obtained from FE analysis and is then fitted with rational function approximation. This chapter shows how to find the poles of the rational function approximation using a technique called Vector Fitting. The model is then constructed by connecting the component low frequency model in parallel with the frequency dependent branch (FDB).

\subsection{Vector fitting}

Vector fitting is a robust numerical method for rational approximation in the frequency domain. It permits to identify state space models directly from measured or computed frequency responses, both for single or multiple input-output systems. This technique was introduced by Gustavsen and Semlyen [53-57].

\subsubsection{The theory of vector fitting}

Vector fitting (VF) approximates the admittance $\mathrm{Y}(\mathrm{s})$ frequency response with rational functions. VF takes as input a column $Y(\mathrm{~s})$ and approximates all of its $\mathrm{n}$ elements simultaneously using an identical set of $\mathrm{N}$ poles [53].

Each element $Y_{j}$ in $h$ becomes 
$Y_{j}(s) \approx Y_{f i t}(s)=\sum_{i=1}^{N} \frac{c_{j i}}{s-a_{i}}+d_{i}+s e_{i} \quad \mathrm{j}=1, \ldots, \mathrm{n}$

Where $c_{i}$ and $a_{i}$ denote the residues and the poles, respectively. Note that the residues depend on $j$, while the poles do not. The terms $d_{i}$ and $e_{i}$ may be specified as zero, if desired. The poles and residues are real or complex conjugate, whereas $\mathrm{d}$ and e are real. The poles and residuals are real or complex conjugate, where $d_{i}$ and $e_{i}$ are real. The VF objective is to find a function that minimizes the following weighted least squares error for the entire vector $Y(s)$ :

$$
\min \left(E=\sum_{j=1}^{n} \sum_{k=1}^{N_{s}} w_{k}\left|Y_{f t i j}\left(s_{k}\right)-Y_{j}\left(s_{k}\right)\right|^{2}\right)
$$

Here $w_{k}>0$ denotes a weight associated with the jth data sample.

It is worthwhile to repeat that the rational function in (6-1) is linear in the unknowns $c_{i}, d_{i}$ and $e_{i}$, but nonlinear in $a_{i}$. As well, the approximation stops at the first order of $\mathrm{s}$ because passive electrical networks are composed of only first-order elements.

VF solves (6-1) sequentially as linear problem in two stages, both times with known poles.

\subsubsection{Pole identification}

Start by stating that $f(s)$ is a rational function, such that $f(s)=\frac{N(s)}{D(s)}$, where the order of the numerator is one greater than the order of the denominator. Our task is to find suitable values for the coefficients of $N(s)$ and $D(s)$ so that this relation is as accurate as possible. 
Select a set of starting poles $\bar{a}_{i}$ in (6-1), and multiple $f(s)$ with an unknown function $\sigma(s)$. The function $\sigma(s)$ has a rational approximation with the same known poles. This gives the augmented problem:

$$
\left[\begin{array}{c}
\sigma(s) f(s) \\
\sigma(s)
\end{array}\right]=\left[\begin{array}{c}
\sum_{i=1}^{N} \frac{c_{i}}{s-\bar{a}_{i}}+d_{i}+s h_{i} \\
\sum_{i=1}^{N} \frac{\widetilde{c}_{i}}{s-\widetilde{a}_{i}}+1
\end{array}\right]
$$

Note that the ambiguity in the solution for $\sigma(s)$ has been eliminated by forcing $\sigma(s)$ to approach unity at high frequencies.

Multiplying the second row in (6-3) by $f(s)$ yields the following:

$$
\left(\sum_{i=1}^{N} \frac{c_{i}}{s-\bar{a}_{i}}+d_{i}+s h_{i}\right)=\left(\sum_{i=1}^{N} \frac{\tilde{c_{i}}}{s-\widetilde{a}_{i}}+1\right) f(s)
$$

or

$$
(\sigma f)_{f i t}(s)=\sigma_{f i t}(s) f(s)
$$

This is a linear equation with unknowns, $c_{i}, d_{i}, e_{i}$, and $\tilde{c}_{i}$. It can be solved as overdetermined problem of the form $A x=b$.

For a given frequency point $s_{k}$ we have:

$$
A_{k} x=b_{k}
$$

Where:

$$
\begin{aligned}
& x=\left[c_{1} \ldots \mathrm{c}_{\mathrm{N}} \mathrm{de} \widetilde{\mathrm{c}_{1} \ldots . \widetilde{\mathrm{c}_{\mathrm{N}}}}\right]^{T} \\
& b=\left[f\left(s_{1}\right) \ldots . . f\left(s_{k}\right)\right]^{T} \\
& A_{k}=\left[\frac{1}{s_{k}-a_{1}} \ldots . . \frac{1}{s_{k}-a_{N}} 1 s_{k} \frac{-f\left(s_{k}\right)}{s_{k}-a_{1}} \ldots . . \frac{-f\left(s_{k}\right)}{s_{k}-a_{N}}\right]
\end{aligned}
$$


$k$ is the total number of sampled frequencies. $k$ must be at least as large as the width of A so that a solution is possible (i.e. $k \geq 2 N+2$ ).

A rational function approximation for $f(s)$ can now be readily obtained from (6-4). This can be shown by writing the sum of partial fraction in (6-4) as a fraction:

$$
(\sigma f)_{f i t}(s)=h \frac{\prod_{n=1}^{N+1}\left(s-z_{n}\right)}{\left(s-\bar{a}_{n}\right)}, \sigma_{f i t}(s)=\frac{\prod_{n=1}^{N+1}\left(s-\tilde{z}_{n}\right)}{\left(s-\bar{a}_{n}\right)}
$$

From $(6-8)$ we get

$$
f(s)=\frac{(\sigma f)_{f i t}(s)}{\sigma_{f i t}(s)}=h \frac{\prod_{n=1}^{N+1}\left(s-z_{n}\right)}{\prod_{n=1}^{N+1}\left(s-\widetilde{z_{n}}\right)}
$$

Equation (6-9) shows that the poles of $f(s)$ become equal to the zeros of $\sigma_{f i t}(s)$. Thus by calculating the zeros of $\sigma_{f i t}(s)$ we get a good set of poles for fitting the original function $f(s)$. Some of the new poles may be unstable. This problem is solved by inverting the sign of the unstable poles real parts.

\subsubsection{Finding the residues}

Once the zeros of $\sigma(s)$ are found, these are used as the new set of known poles, $a_{i}$, and then the (6-1) is solved in a manner similar to the technique used to obtain $c_{i}, d_{i}$, and $e_{i}$. This is again a linear least-squares problem of the form $A x=b$.

\subsubsection{Iteration}

Step 6.3.1 and 6.3.2 may have to be repeated several times with the new poles as starting poles, in order for the method to converge. When convergence is achieved, $\sigma(s)$ becomes unity. 
Thus vector fitting solves Eq. (6-1) as follows:

1. Chose a set of starting poles $\bar{a}_{1} \ldots \bar{a}_{N}$.

2. Solve (6-4) with respect to $c_{i}, \tilde{c}_{i}, d, h$.

3. Calculate the poles $a_{1} \ldots . . a_{N}$ of $\mathrm{Y}(\mathrm{s})$ as the zeros of $\sigma(s)$.

4. Solve (6-4) with respect to $c_{i}, d, h$.

5. If necessary repeat procedures from 1-4 with the new poles as starting poles until constraints given by $(6-2)$ is minimized.

\subsubsection{Synthesization by electrical network}

The rational function given by (6-1) can be represented by an equivalent electrical network as shown in Fig. 6-2

$R_{o}$ and $C_{o}$ are calculated as

$$
C_{o}=e, R_{o}=\frac{1}{d}
$$

Each real pole gives an RL-branch

$$
R_{1}=-\frac{a}{c}, \mathrm{~L}_{1}=\frac{1}{c}
$$

Each complex conjugate pair $\frac{c^{\prime}+j c^{\prime \prime}}{s-\left(a^{\prime}+j a^{\prime \prime}\right)}+\frac{c^{\prime}-j c^{\prime \prime}}{s-\left(a^{\prime}-j a^{\prime \prime}\right)}$

Gives an RLC branch

$$
\begin{aligned}
& L=\frac{1}{2 c^{\prime}} \\
& R=\left(-2 a^{\prime}+2\left(c^{\prime} a^{\prime}+c^{\prime \prime} a^{\prime \prime}\right) L\right) L \\
& \frac{1}{C}=\left(a^{\prime 2}+a^{\prime 2}+2\left(c^{\prime} a^{\prime}+c^{\prime \prime} a^{\prime \prime}\right) R\right) L
\end{aligned}
$$




$$
G=-2\left(c^{\prime} a^{\prime}+c^{\prime \prime} a^{\prime \prime}\right) C L
$$

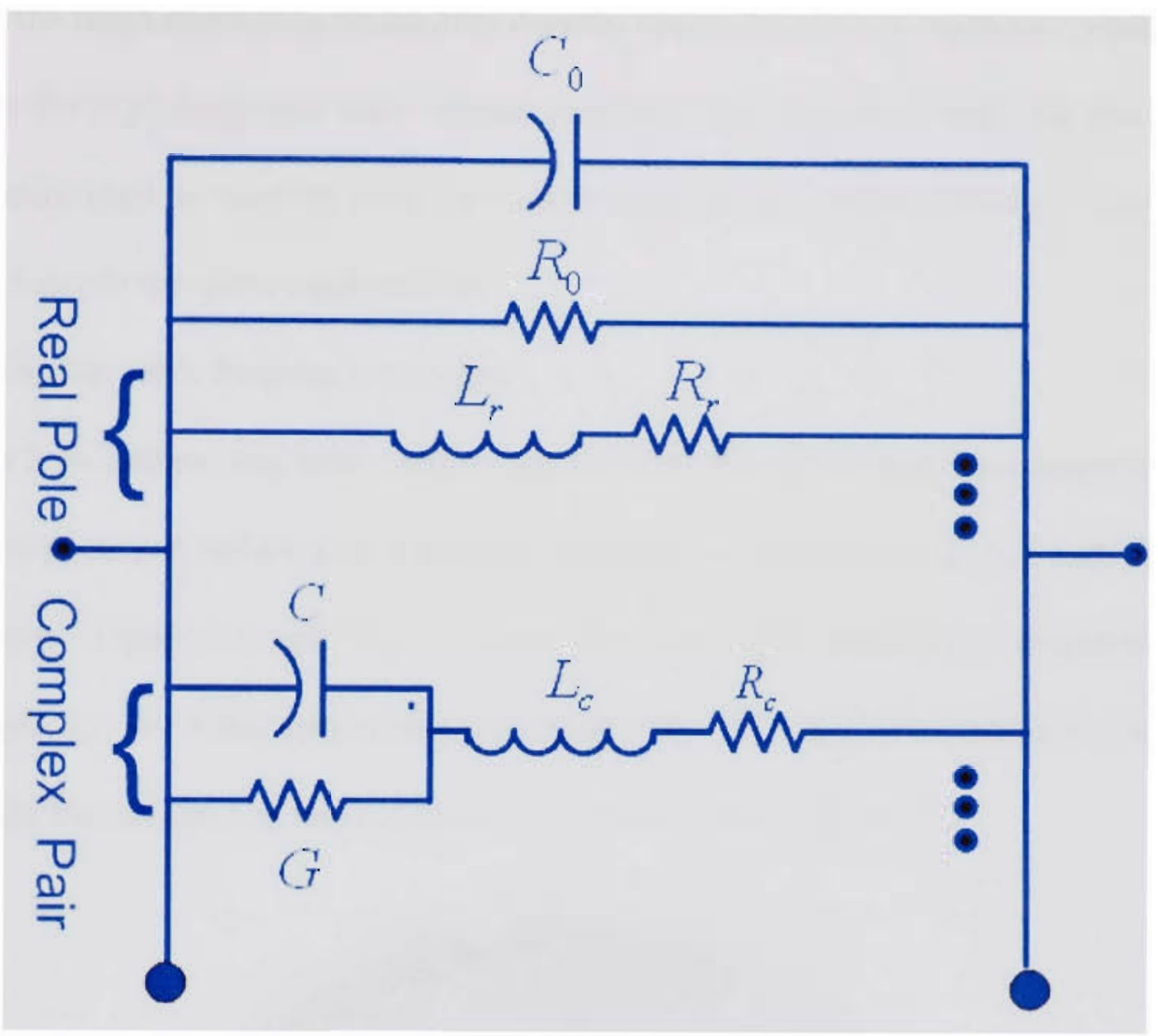

Fig. 6-2 Synthesization by electrical network

\subsection{Cable frequency dependent modeling}

The cable frequency response was obtained from FE magnetodynamic analysis. The mesh is adjusted in coordination to the skin depth. The mesh size should be less than the skin depth.

\subsubsection{Cable frequency response}

A 2-D FE magnetodynamic problem is solved to calculate the resistance and inductance of the cable as a function of frequency. For calculation of these parameters, the eddy current effects in the conductors are considered. The conductors, resistivity and unit permeability were assigned. All other materials were set to properties of air. The 
time harmonic solution with nonlinearity is obtained by assigning different permeability values to the mesh depending on the flux density vector. In order to mesh the conductor's region for the high frequency eddy current problem, care was taken such that the size of the elements used is smaller than the wavelength of the PWM excitation frequency. Figure 6.3 shows the cable mesh details.

\subsubsection{Fitting the cable frequency response}

The VF procedure was then used to approximate the cable frequency response. The cable resistance and inductance frequency response is shown in Fig. 6.4 and Fig. 6.5 respectively. Figure 6.6 and Fig 6.7 show the fitted cable admittance magnitude and phase angle for six order approximation respectively. The dot line represents the fitted value while the continuous line represents the values obtained from $\mathrm{FE}$.

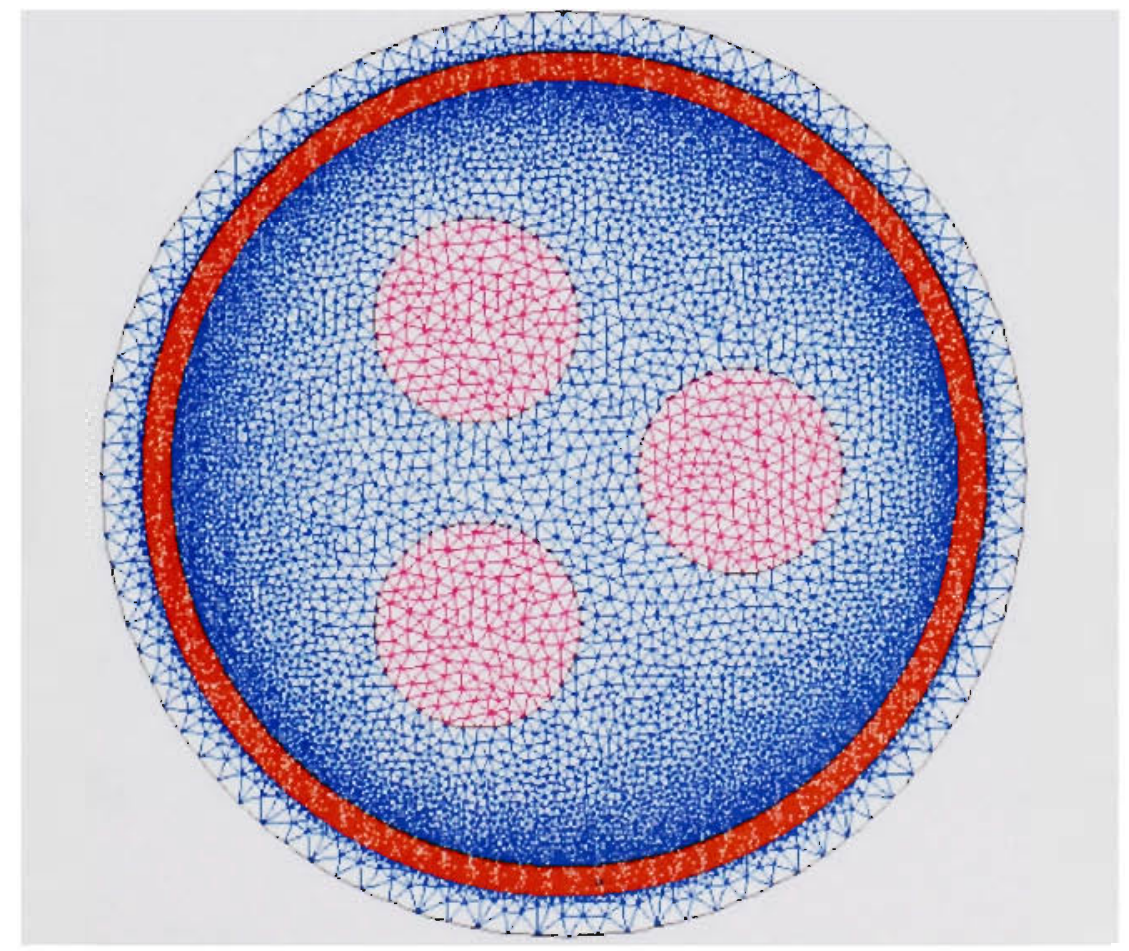

Fig. 6.3 The used cable geometry and the mesh details 


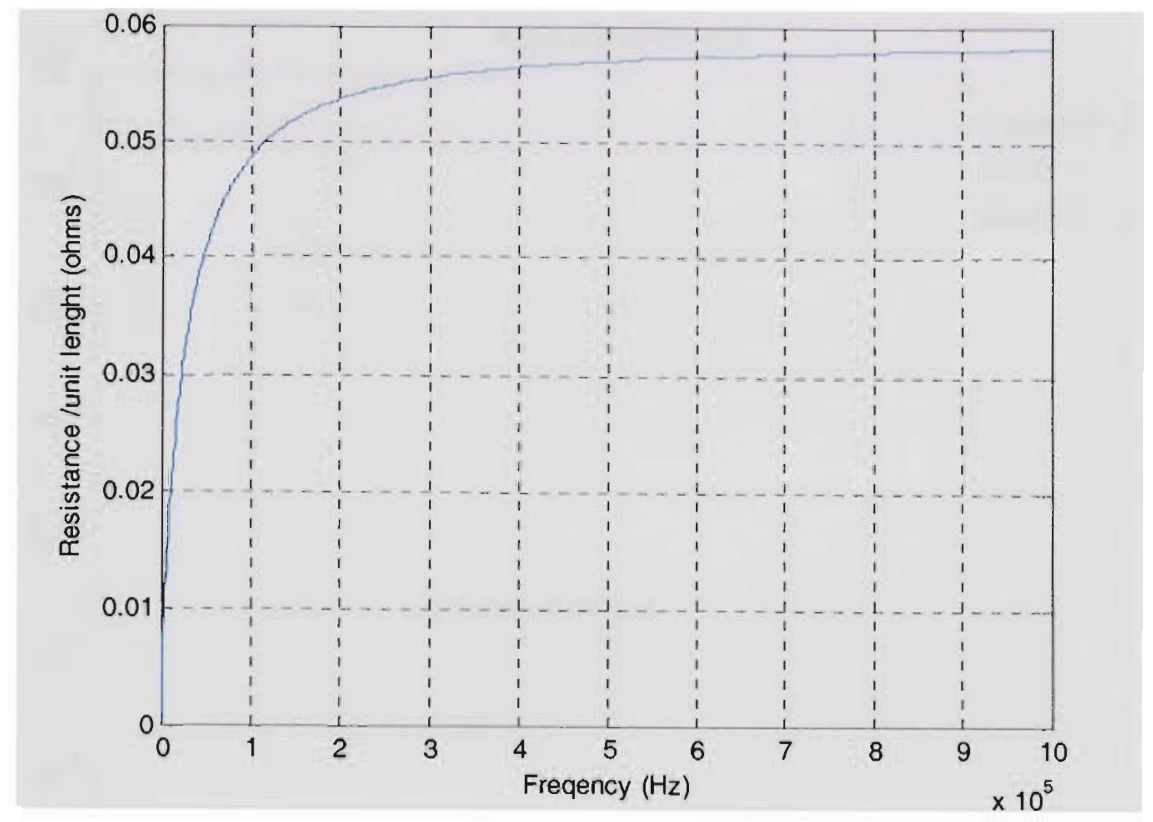

Fig. 6.4 The frequency dependence of the cable resistance

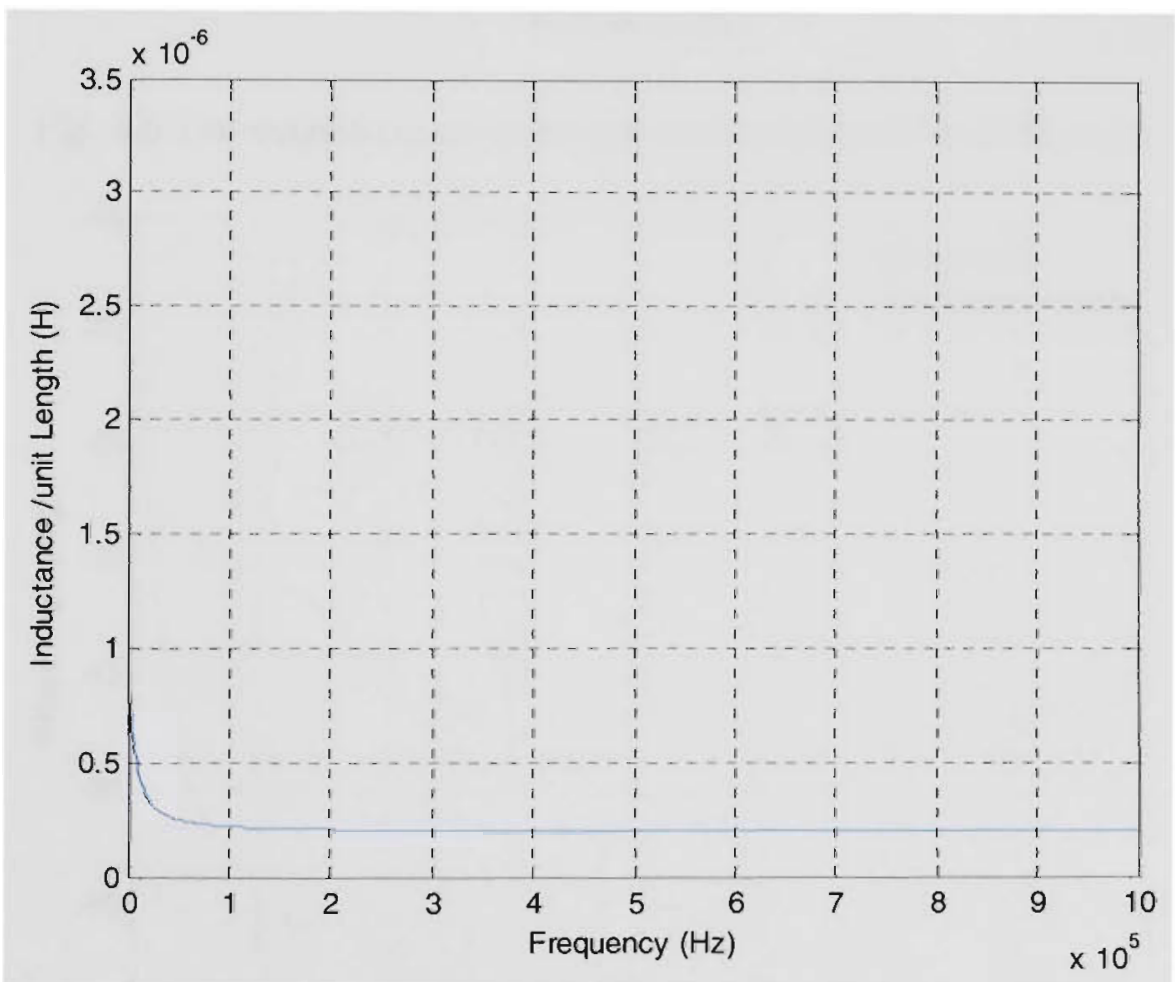

Fig. 6.5 The frequency dependence of the cable inductance 


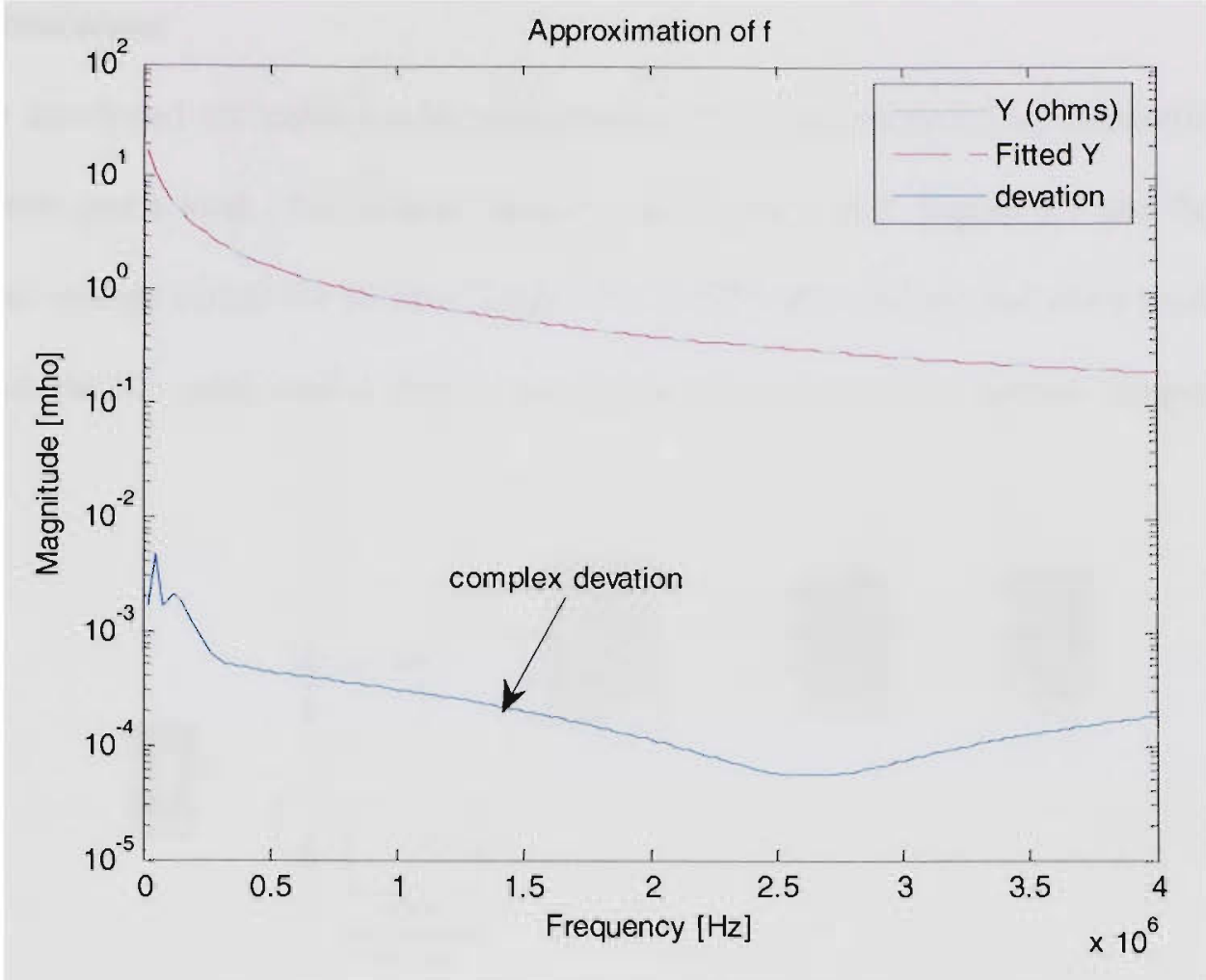

Fig. 6.6 The magnitude six order approximation result and the error

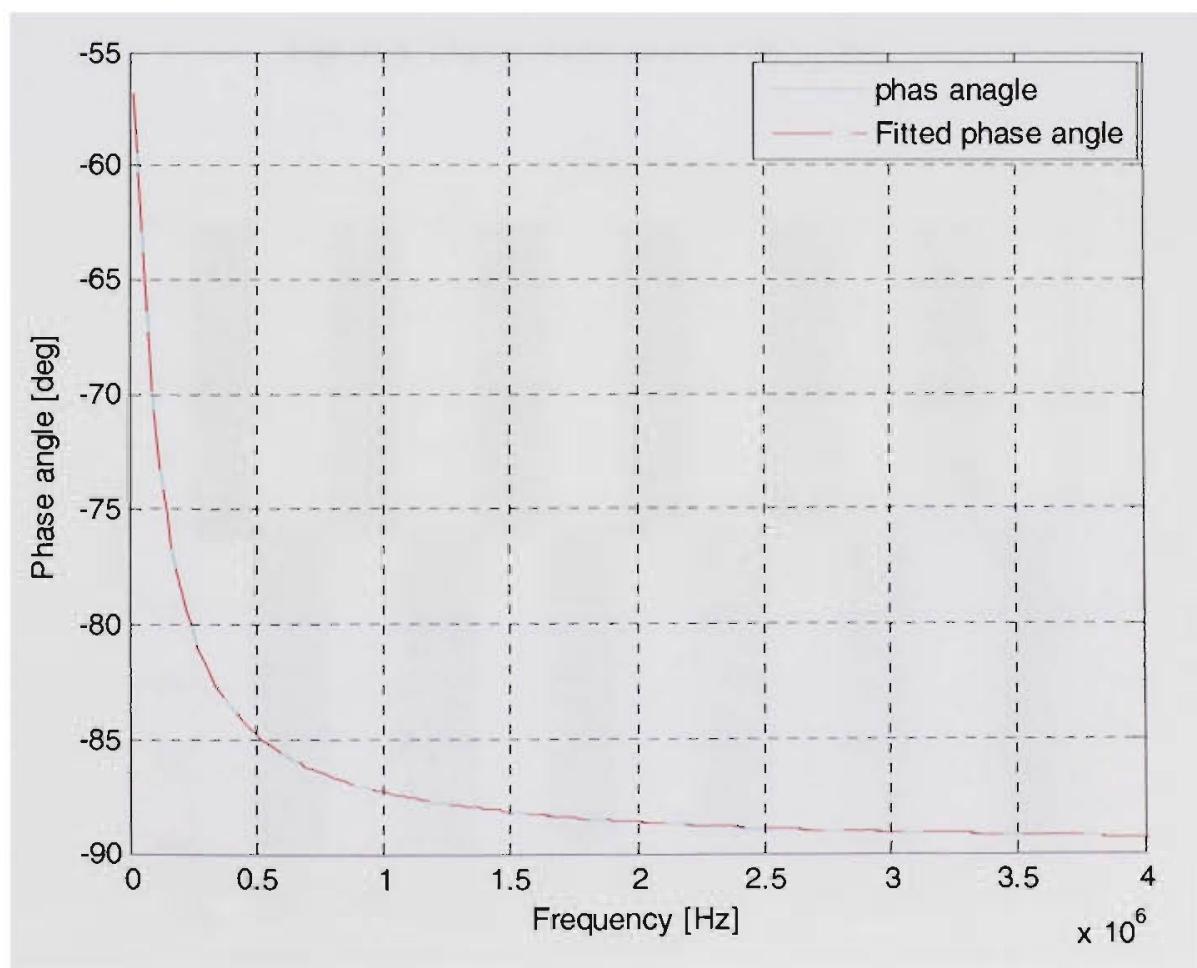

Fig. 6.7 The phase angle six order approximation of the cable impedance 


\subsubsection{Simulations}

The developed HF cable model performance was then evaluated by connecting it to an inverter and a load. The system used is shown in Fig. 6.8. Figure 6.9 and Fig. 6.10 show the voltage across the inverter output due to HF cable and normal cable model. It's clear that the HF cable model shows more harmonics compared to normal lumped cable model.

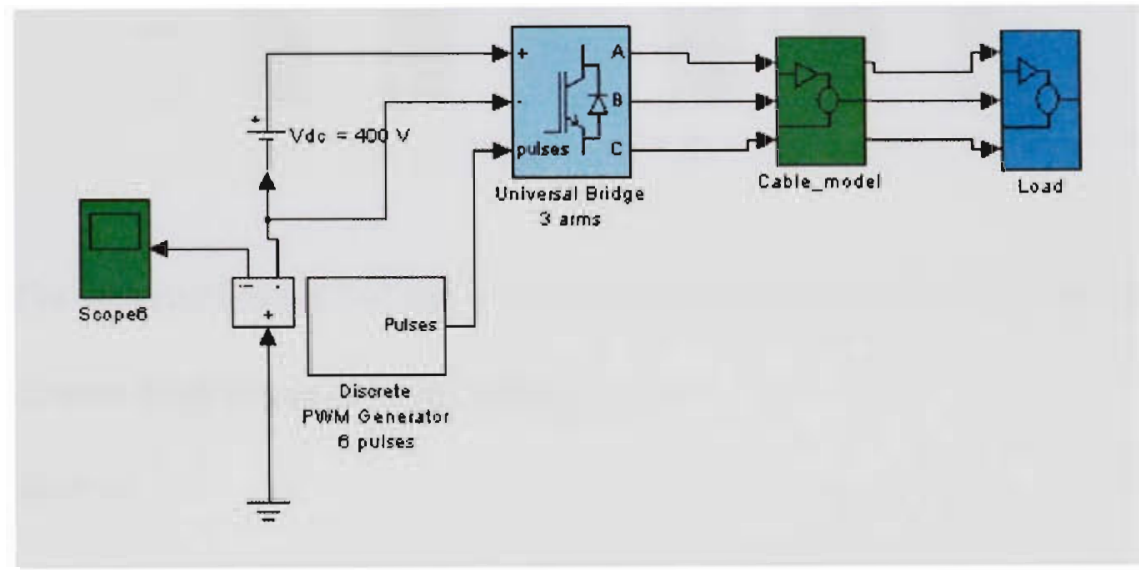

Fig. 6.8 The system used in the study

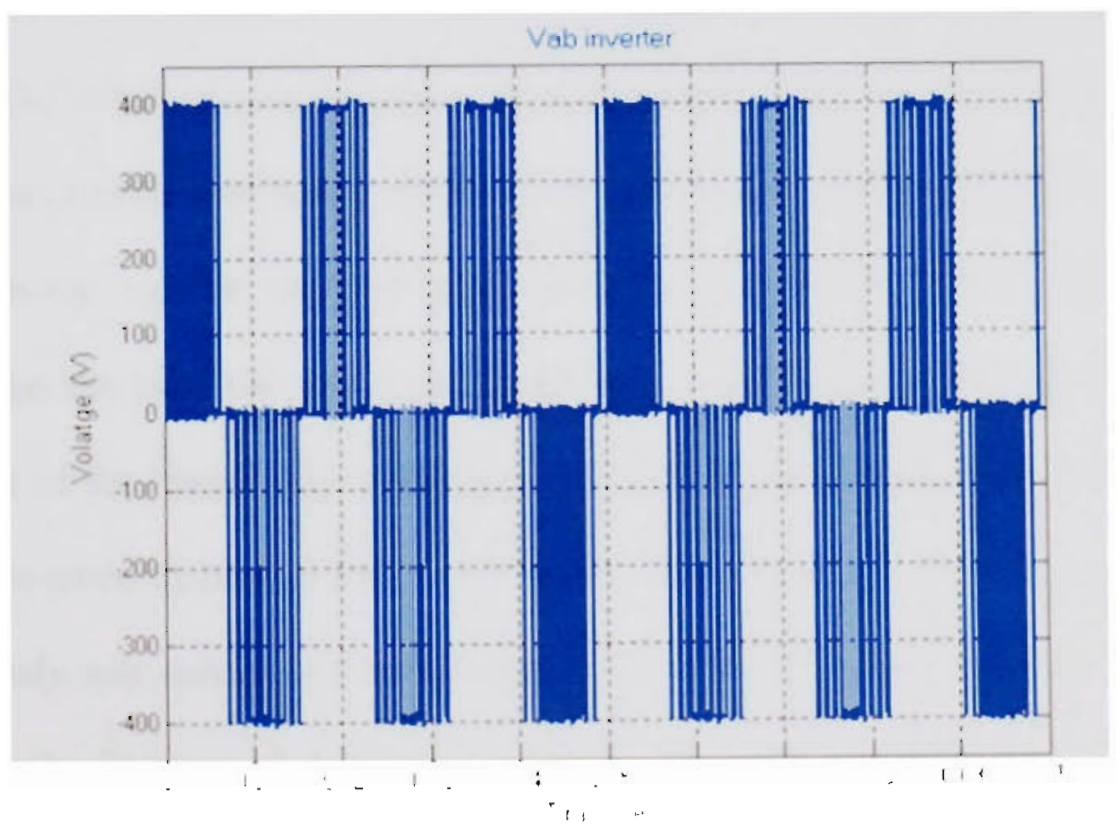

Fig. 6.9 The inverter out voltage when connected to the developed HF cable model 


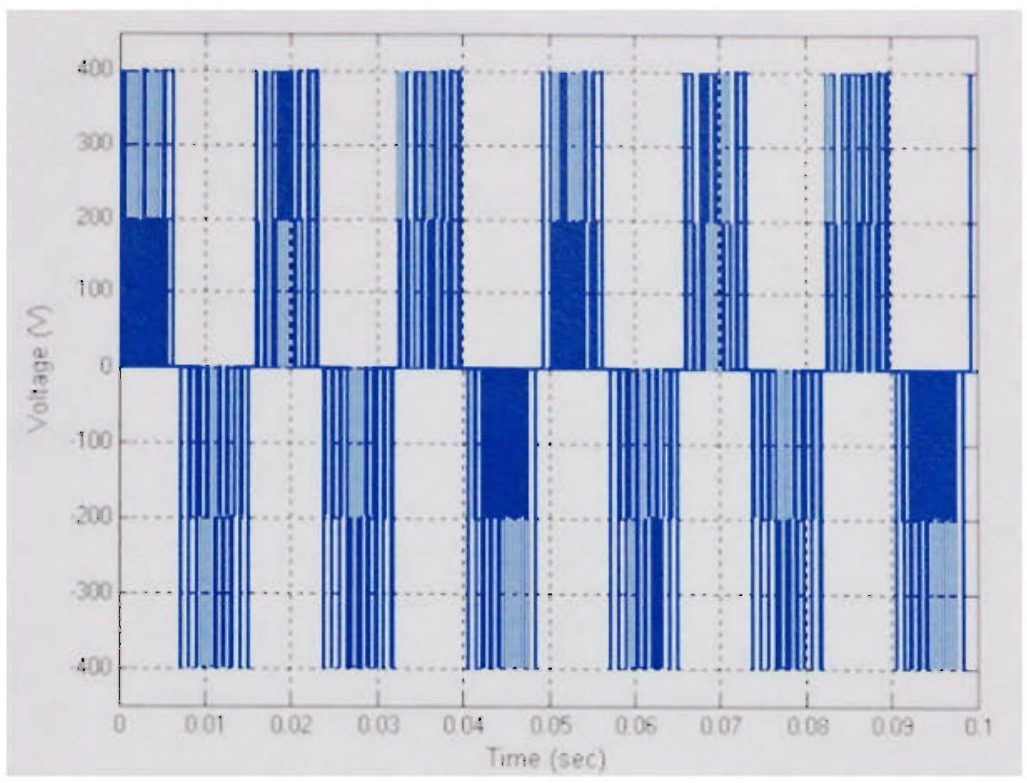

Fig. 6.10 The inverter output voltage when connected to the normal lumped cable model

\subsection{Transformer high frequency modeling}

\subsubsection{Introduction}

Modeling of power transformer represents a challenge to engineers. There are different models for transformers for various contingencies. These models were designed to include the effect of core saturation, ferroresonance, hysteresis, and insulation issues. The increase utilization of power electronic devices (e.g. inverter, rectifier) and nonlinear loads in power system required more accurate transformer models that take into consideration the time variant of the transformer parameters as well as the frequency dependence of the transformer parameters. The transformer model accuracy will affect the ability to correctly predict the system harmonics and the total harmonic distortion.

This study was carried out on a single phase $125 \mathrm{kVA}$, shell type transformer with sandwich coils. Figure 6.11 shows the transformer geometry and the mesh details used in the study. 


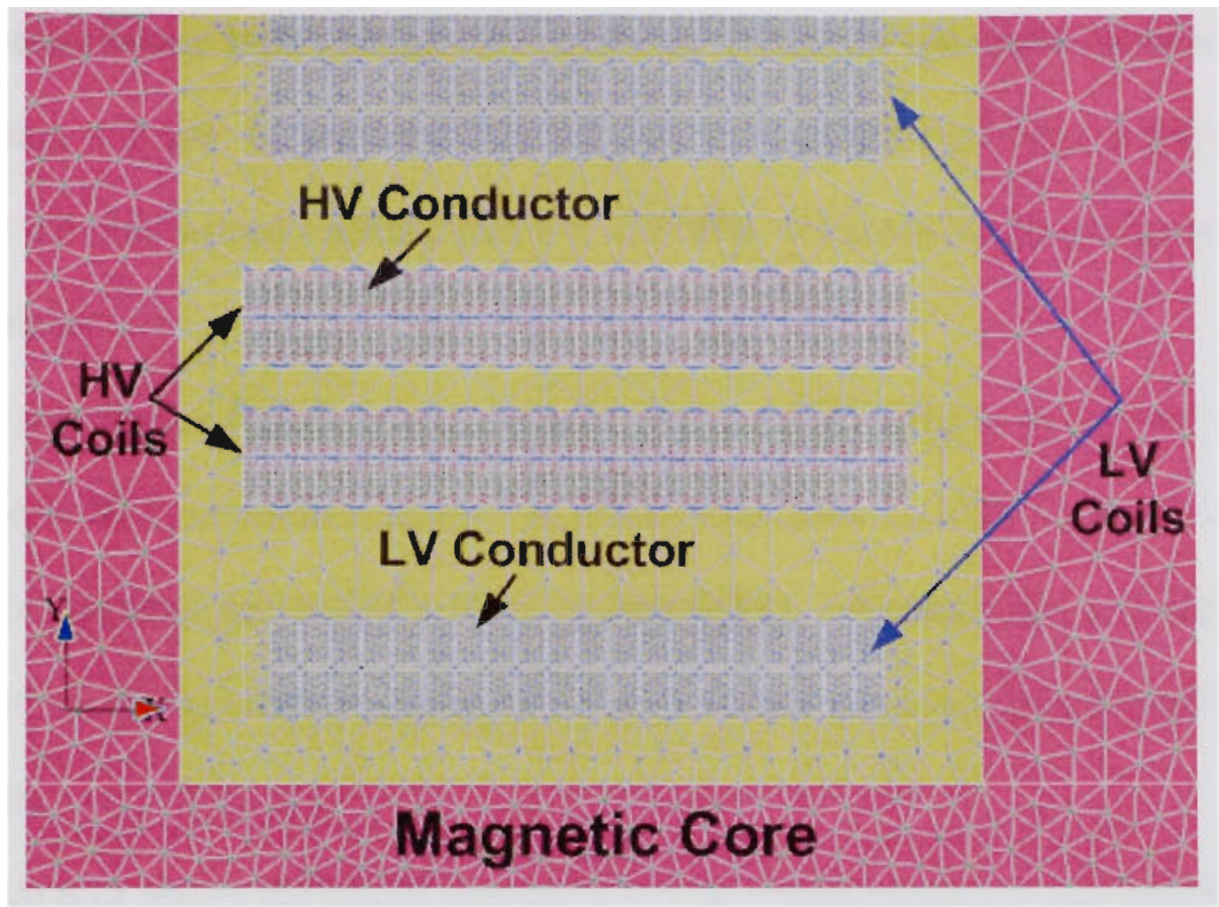

Fig.6.11. The used transformer geometry

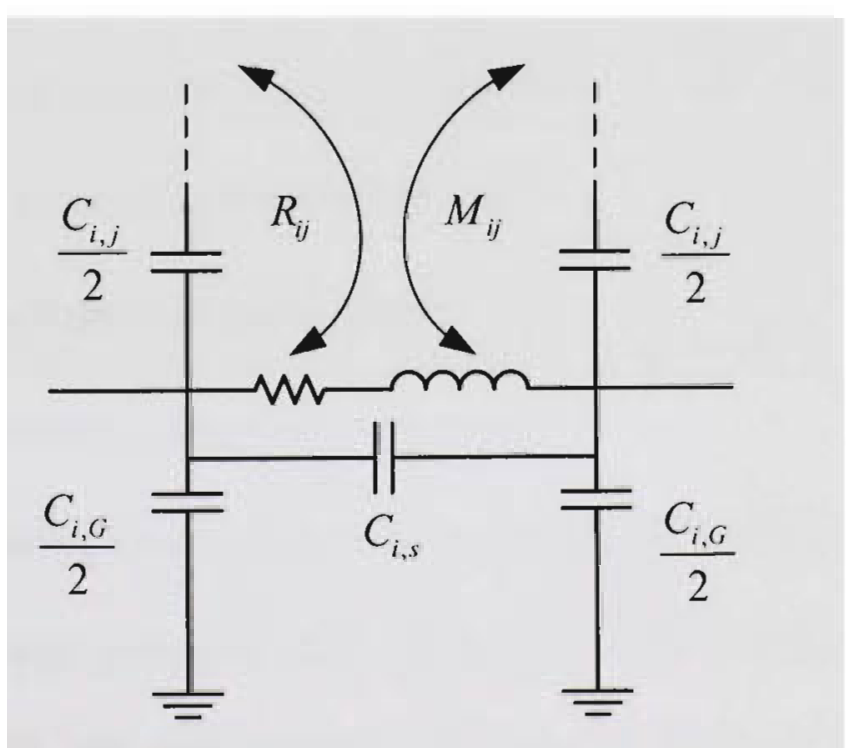

Fig.6.12. Lumped parameter equivalent of one winding 
The transformer high voltage side conductor comprises two paper covered stripes each $7 \mathrm{~mm} \times 2 \mathrm{~mm}$ in parallel while the low voltage conductor has 4 stripes each $7 \mathrm{~mm} \times 4.5 \mathrm{~mm}$ wound together with a $1 \mathrm{~mm}$ strip of fibrous insulation between each set of 4 strips.

The transformer high frequency behavior was modeled using large number of multinodal, linear RLC networks connected in series. Each linear network represents one turn. Each RLC network consists of a linear resistance (R), inductance (L), and capacitance (C) based on the transformer geometry and transformer materials magnetic and electric properties.

Figure 6.12 shows the circuit equivalent of one winding element. Mutual effects are shown for both inductance and resistance. Mutual capacitances to other windings and to the ground are shown as well as series capacitance across the winding. The different elementary parameters are as follows:

$R_{i}=$ winding resistance at the selected frequency (DC Value plus the skin effect)

$L_{i}=$ winding self inductance (Skin effect Included)

$C_{s, i}=$ series capacitance along the winding itself

$C_{i, G}=$ Capacitance from winding to ground

$M_{i, j}=$ Mutual inductance between winding $i$ and $j$

$R_{i, j}=$ losses in winding $i$ due to the current in winding $j$ (Proximity effect)

Due to the geometry symmetry, only one quarter of the transformers geometry is modeled. Under PWM operating condition the resistance, inductance and capacitance values varies from the low frequency operation. The windings have skin and proximity 
effects which cause resistance to be much higher than the low frequency resistance. The inductance value decreases with an increase in the operating frequency while the capacitance effect comes into the picture at high frequencies. Also with the PWM operation, the transformer's winding capacitance is distributed between several parts of the winding (turn to turn and turn to ground). Hence, to obtain accurate values of the resistance, self and mutual inductances and various capacitances, finite element analysis is used. The mesh element size is selected in coordination with the skin depth (depth of penetration).

\subsubsection{Inductance Calculation}

If several conductors are current excited at the same time, the computed inductance for a conductor is a global inductance L. This includes the self inductance of the chosen conductor as well as the mutual inductances due to other conductors. From the voltage and current in the source, the reactance is calculated.

The calculated inductances here are the transformer's apparent inductances. The variation of global inductance for source with frequency is shown in Fig. 6.13. The Figure shows that the primary inductance value decreased by $50 \%(60 \mathrm{~Hz}-50 \mathrm{kHz})$.

\subsubsection{Resistance calculation}

A 2-D FE magnetodynamic problem is solved to calculate the resistance and inductance of the transformer as a function of frequency. For calculation of these parameters, the eddy current effects in both conductor and iron core were considered. To include these effects, two properties were assigned to the iron material; nonlinear permeability and resistivity. In the case of conductors, resistivity and unit permeability were assigned. All other materials were set to properties of air. 


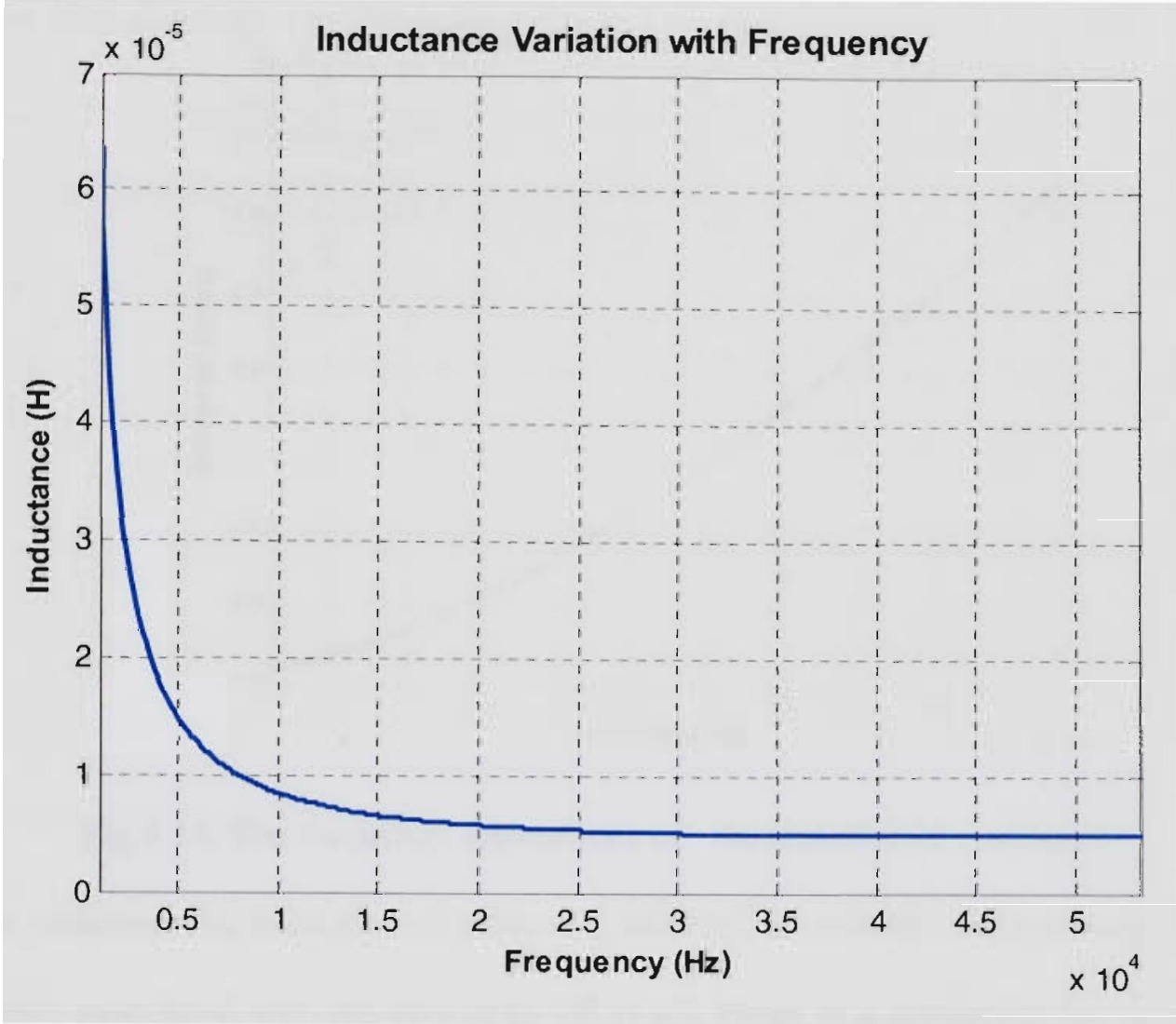

Fig.6.13. The frequency dependence of the transformer inductance

The time harmonic solution with nonlinearity is obtained by assigning different permeability values to the mesh depending on the flux density vector. In order to mesh the conductor's region for the high frequency eddy current problem, care was taken such that the size of the elements used is smaller than the wavelength of the PWM excitation frequency.

For overvoltage studies, corresponding to $0.2 \mu$ s rise time of PWM supply wavefront, the frequency range of the FE analysis was chosen to be $60 \mathrm{~Hz}-10 \mathrm{MHz}$. For studying the effect of different switching frequencies, $10-30 \mathrm{kHz}$ PWM frequencies were used in the simulation. 


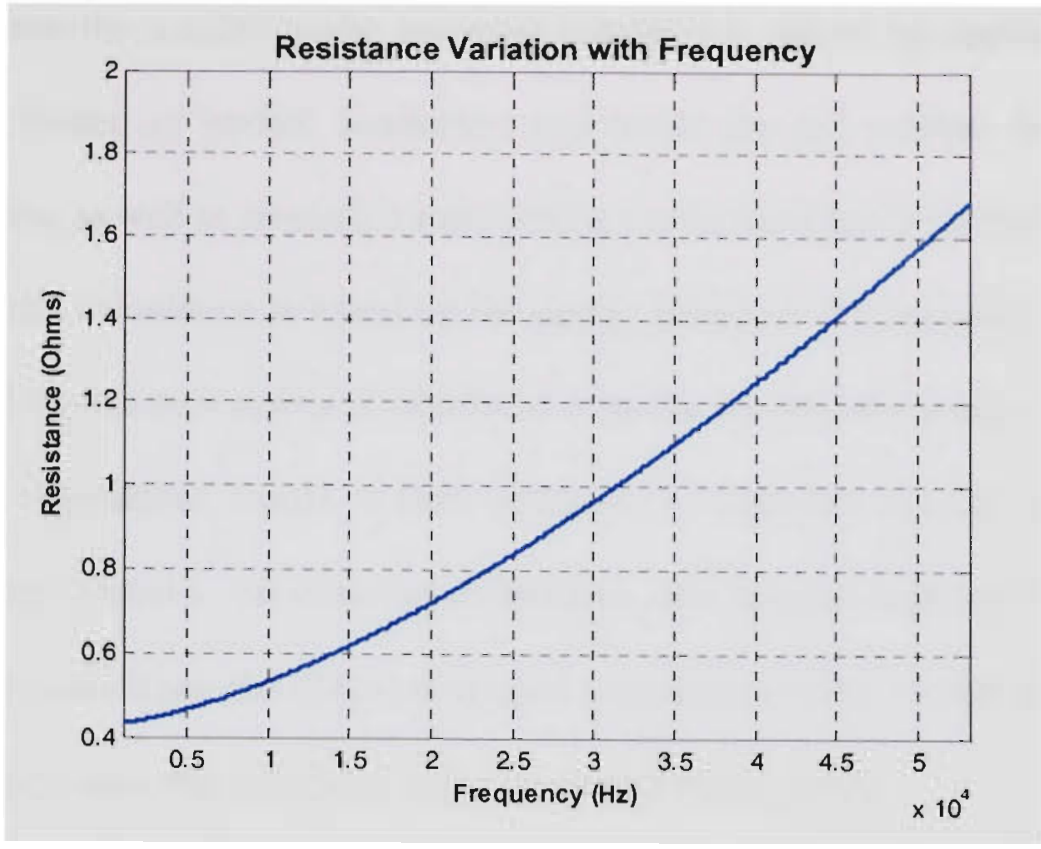

Fig.6.14. The frequency dependence of the transformer resistance

The simultaneous excitation of turns is performed to consider the proximity effects. The losses associated with the proximity effect are losses in a conductor due to currents in all other conductors. Proximity effects are far more than skin effects while calculating the resistance values at high frequency operation.

After solving coupled field and circuit problem simultaneously, the voltage in the source is obtained. From the voltage and current phasors in the source, the resistance is calculated. The variation of resistance with the source frequency is shown in Fig. 6.14. The Figure shows that the resistance value increased by $300 \%$ when the supply frequency increase from $60 \mathrm{~Hz}$ to $50 \mathrm{kHz}$.

\subsubsection{Capacitances calculation}

The electrostatic problem is solved for the calculation of capacitances as capacitances are a function of geometry rather than frequency. An electrostatic analysis is assumed to be a linear analysis that means the electric field is proportional to the applied voltage. The 
analysis determines the electric scalar potential distribution caused by applied voltage. The turns were treated as perfect conductors and hence are not meshed from inside. Boundaries of turns as well as insulation surrounding the turn are finely meshed.

The capacitance calculation is based on the energy principle. By applying voltage on turns, the ground capacitance matrix is calculated from the stored static energy.

The lumped capacitance matrix is then obtained. To calculate lumped capacitance matrix, charges are related to the potential differences. The lumped capacitance matrix is obtained for coil turns. Then the obtained lumped capacitance value is multiplied by the mean length of turn since the calculated value is per unit meter length.

\subsubsection{Model Reduction Technique}

This technique was developed by F. de leon and A. Semlyen [124]. This reduction technique is used to reduce the order of the distributed parameter winding. In this technique, all the nodes other than terminal nodes are eliminated. A nodal model is created in which half of each capacitance is shifted towards two ends of the conductor and then the nodal capacitance matrix is formed from the conductor capacitance matrix by recognizing which elements are connected to each node. The resulting matrix is of the order $2 \mathrm{~N}$ with the general terms given by:

$$
\begin{aligned}
& C_{2 i-1,2 j-1}=C_{2 i, 2 j}=1 / 2 C_{i, j}^{\text {turn }} \\
& C_{2 i-1,2 j}=C_{2 i, 2 j-1}=0
\end{aligned}
$$

To reduce the order of model, series connected conductors in one slot are lumped to form sections. In short, the process is shifting of internal capacitances towards preestablished section nodes. Once the capacitances are moved to section nodes, conductor 
inductances and resistances are lumped together. The entire process is summarized in Fig. 6.15 .

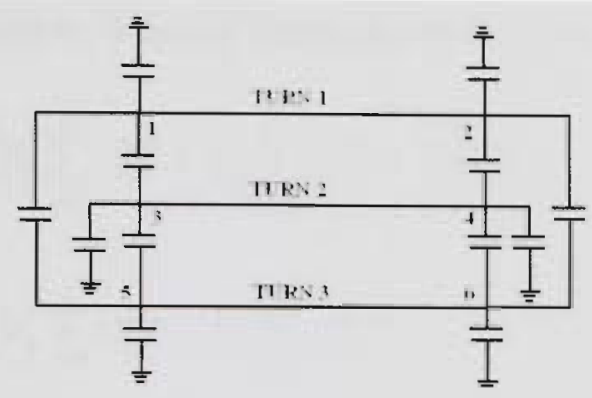

(a) Turn-to-turn model

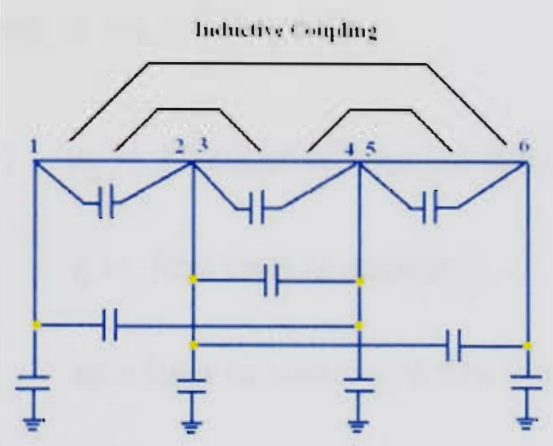

(b) First Lumping step

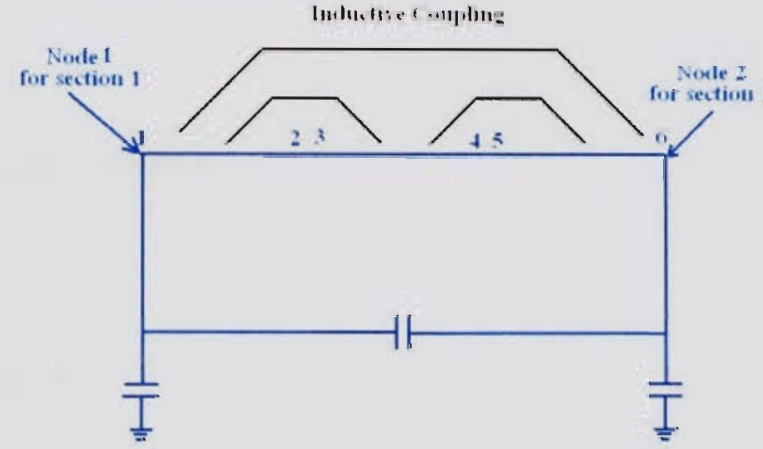

(c) Capacitance Moved to the section nades

Fig. 6.15 The reduction Technique

There is no need to write explicitly the matrix $C_{\text {node }}$ of order $2 \mathrm{~N}$ since all the needed information is contained in the matrix $C^{\prime \prime \prime \prime n}$, which is of order N. By using equation (6-1) and the definitions of self $\left(C_{i i}\right)$ and mutual $\left(C_{i j}, i \neq j\right)$ capacitances we have,

$$
\begin{aligned}
& C_{1,1}^{\mathrm{sec}}=C_{1.1}^{\text {turn }}+\frac{1}{2}\left(C_{1,2}^{\text {urm }}+C_{2.1}^{\text {turn }}\right)+\frac{1}{2} C_{2.2}^{\text {t(urn }} \\
& C_{2.2}^{\mathrm{sec}}=C_{3.3}^{\text {turn }}+\frac{1}{2}\left(C_{3.2}^{\text {unrn }}+C_{2,3}^{t u n}\right)+\frac{1}{2} C_{2,2}^{\text {turn }} \\
& C_{1.2}^{\mathrm{sec}}=C_{2,1}^{\text {urrn }}=C_{1,2}^{\text {urun }}+\frac{1}{2}\left(C_{1,2}^{\text {turn }}+C_{2,1}^{\text {uwn }}\right)
\end{aligned}
$$


The procedure described above can be generalized for any odd number of turns per section, any number of sections per winding.

The general equations to form a winding are as follows:

$$
\begin{aligned}
& C_{i i \text { node }}^{\mathrm{sec}}=\sum_{i=i_{i}}^{l_{i}} \sum_{j=i_{i}}^{l_{i}} C_{i j}^{\text {turn }}+1 / 2 \sum_{i=i_{i}}^{l_{i}} C_{i, m}^{\text {turn }}+1 / 2 \sum_{j=i_{i}}^{l_{i}} C_{m, j}^{\text {turn }}+1 / 2 C_{m, m}^{\text {turn }} \\
& C_{i j \text { node }}^{\mathrm{sec}}=\sum_{i=i_{i}}^{l_{i}} \sum_{j=i_{j}}^{l_{i}} C_{i j}^{\text {turn }}+1 / 2 \sum_{i=i_{i}}^{l_{i}} C_{i, m}^{\text {turn }}+1 / 2 \sum_{j=i_{j}}^{l_{i}} C_{m, j}^{\text {turn }}
\end{aligned}
$$

Where, $l_{i}=i_{i}+\frac{1}{2}\left(w_{k}-1\right)-1$

$$
\begin{aligned}
& w_{k}=\text { Number of lumped turns in section } \mathrm{k} \\
& i_{i}=\text { first turn in section } \mathrm{i} \\
& m=\text { turn in section of this section }
\end{aligned}
$$

From (6-21) and (6-22), we can see that the reduction process is simply carried out by the addition of elements in the turn capacitance matrix $C^{\text {turn }}$ by blocks. The boundaries for forming the blocks are the turns located at the beginning, at the center and at the end of section to be formed. Each section produces four entries in the node capacitance matrix for sections $C_{\text {node }}^{\text {sec }}$. Once the capacitances have been moved to the ends of the section become connected in series. In this case the equivalent inductance is the sum of all element self and mutual inductance.

\subsubsection{Model Description}

Figure 6.16 shows high frequency phase variable model of transformer. It is constructed by connecting high frequency branch in parallel with the power frequency model. The Power frequency part represent the transformer for power frequency 
operation while high frequency winding branch represents the transformer high frequency dynamic behavior to account for overvoltages or spikes in the current waveforms.

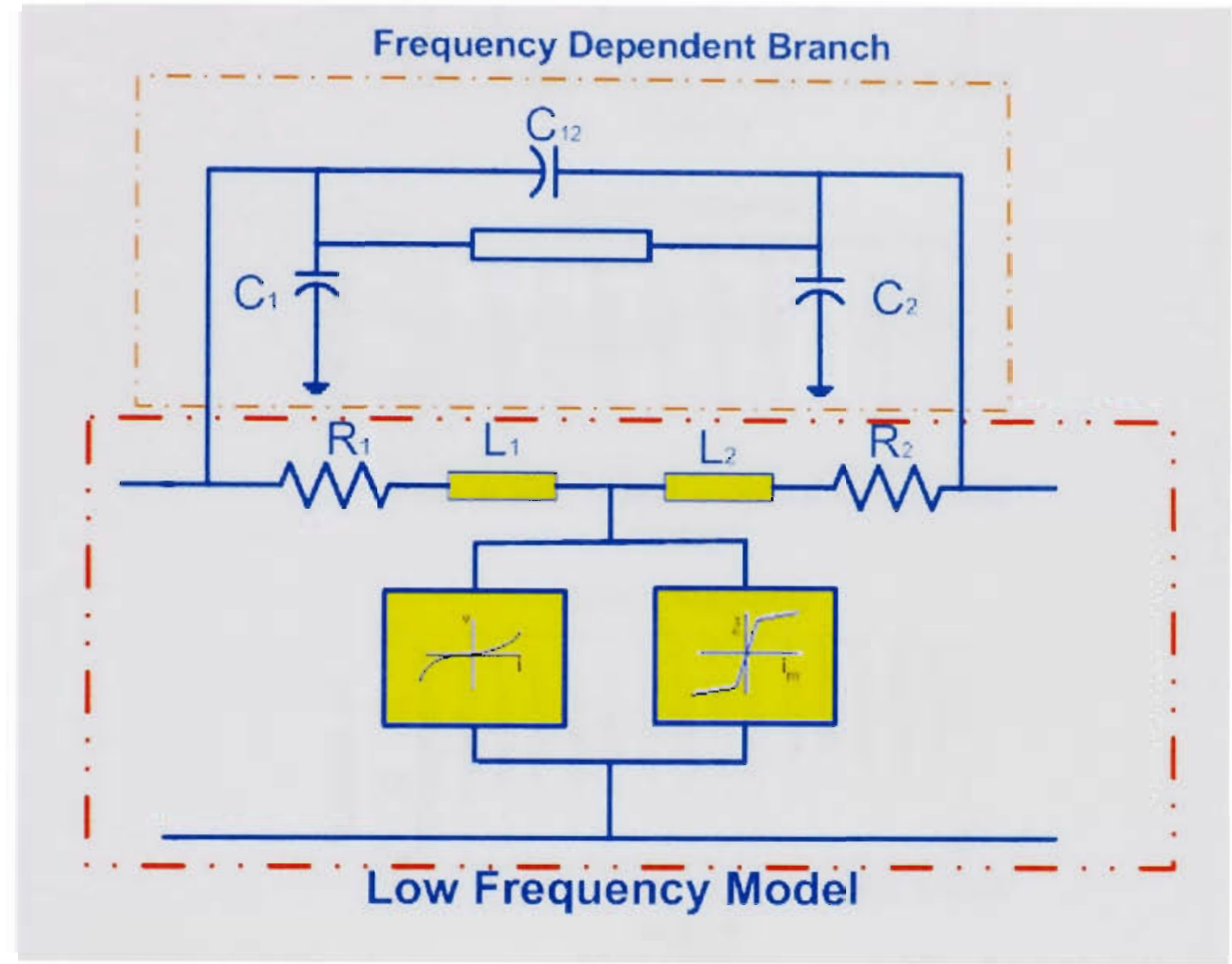

Fig. 6.16 High frequency phase variable model of transformer

\subsubsection{Simulation results}

The developed high frequency physical phase variable model of the transformer is tested with constant frequency inverter and cable to study the effect of different switching frequencies implemented in Simulink.

The obtained high frequency current profile is shown in Fig. 6.17. It can be seen that the proposed high frequency model can reflect the spikes due to the PWM excitation. Pulsations in the waveforms are the result of PWM action while spikes are the result of high PWM switching speeds. 

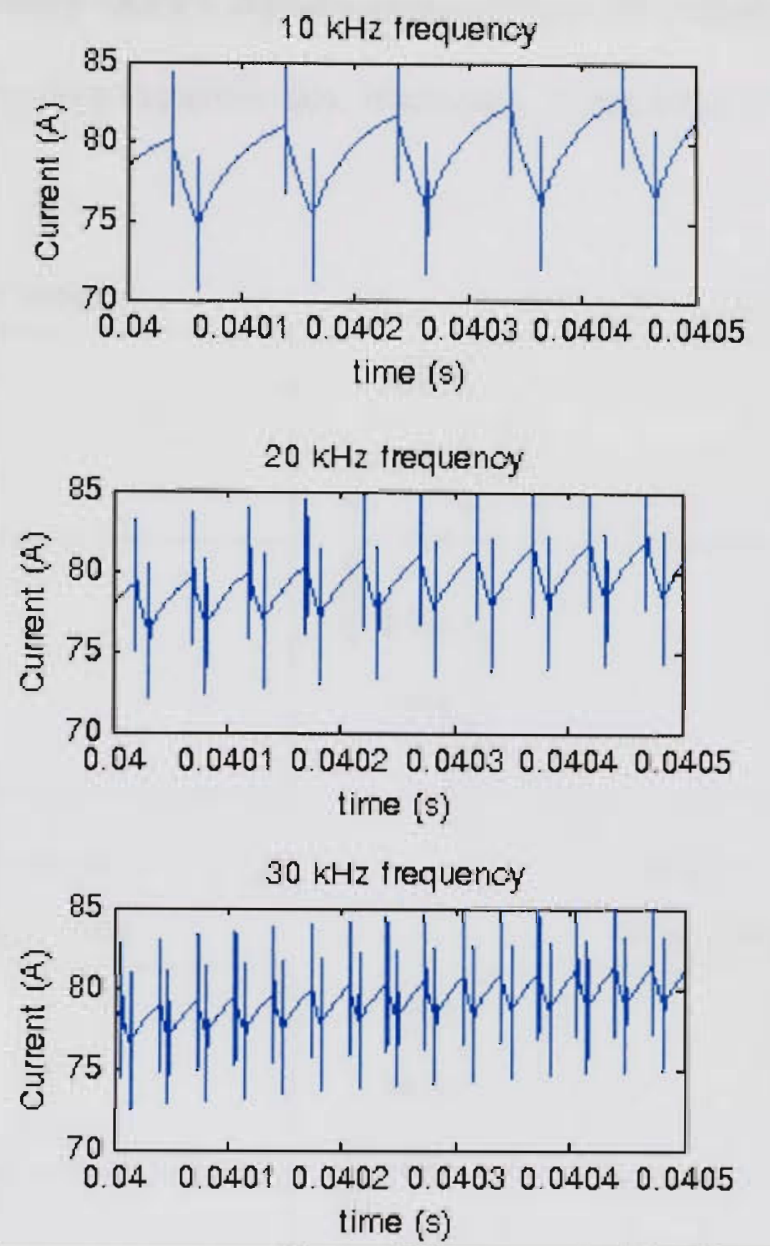

Fig. 6.17 Primary current profile at different switching frequencies

The number of source current spikes is increased with the increase in supply switching frequency. This is obvious as the spikes appear at every transition of PWM supply. The increase in the magnitude of the spike is marginal as both capacitance and applied voltage are constant during all switching frequency operation. With increase in the frequency, high frequency branch provides low resistance path to high frequency components. As low and high frequency models are connected in parallel, high frequency components passing to the low frequency path are reduced. If high frequency branch is removed, the spikes inherent in the PWM operation can not be obtained by low frequency 
model. Thus high frequency branch represents accurately the transformer under PWM operation and take into consideration the frequency dependence of the transformer parameters.
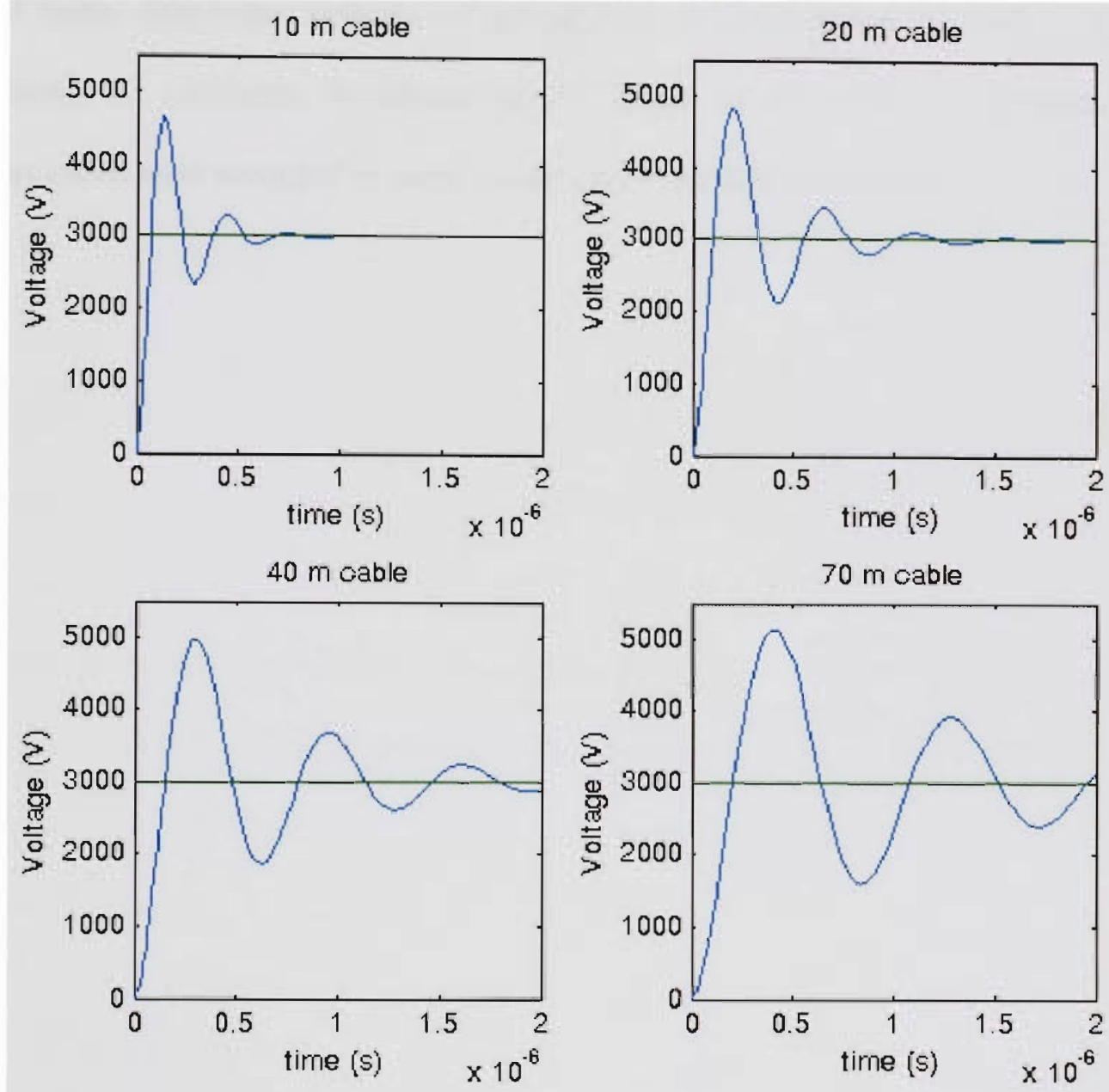

Fig. 6.18 Primary current profile at different switching frequencies

Also the interaction between the inverter, cable and transformer is studied to predict the overvoltage transients. A high frequency cable model developed in the previous section was used. These voltage transients are mainly due to the steep voltage rise times of PWM inverter as well as impedance mismatch between cable characteristic (surge) impedance and transformer input impedance. 
The overvoltage results were obtained for different cable lengths. As the length of the cable increases, the overvoltage peak value also increases as shown in Fig. 6-18. For comparison purpose, the magnitude of PWM voltage is also shown which is equal to 3000 volts. Since the velocity of propagation is independent of cable length, the frequency of oscillation is reduced as the length of the cable is increased. Similar observations were recorded by other authors utilizing different models. 


\section{Chapter 7: Wavelets Transform}

\subsection{Introduction}

The word wavelet is derived from the French word "ondelette", meaning small wave. The beginnings of the wavelets transform as a specialized field can be traced to the work of Grossman and Morlet [197-200]. Their motivation in studying wavelet transforms was provided by the fact that certain seismic signals can be modeled suitably by combining translations and dilations of a simple, oscillatory function of finite duration called a wavelet.

Wavelets are oscillatory functions that are localized in both time and frequency domains. A signal representation based on these localized oscillations is highly desired because the wavelet coefficients convey details in time and frequency. A small coefficient means there is little variation in the function or signal in the vicinity of that particular small oscillation, whereas a large coefficient indicates that there is appreciable change in the signal or function. Analysis of the coefficients allows features in the function or signal to be identified.

The short-term Fourier transform (STFT) is a time-frequency analysis technique which used a specific window to divide the time-frequency plane into uniform divisions. The window will suppress the analyzed signal outside a certain region and therefore allow the Fourier transform to represent the signal in both time-frequency domains. For nonstationary signals the accuracy of the STFT for extracting signal time/frequency features is highly limited by the fixed window length. If the window is designed for low frequencies the long window will not be suitable for high frequencies contents within the waveform. On the other hand, for a window designed for high frequencies the window 
length will not be long enough to handle low frequencies and will result in excessive frequency distortion of the signal spectrum. Therefore the STFT with its fixed width window is not readily adaptable for capturing the nonstationary signals characteristics [59-63].

As Fourier transform, the wavelet transform decompose a given function into a series in term of a basis function. However, unlike Fourier transform which uses complex exponentials as a basis function, wavelet transform uses the dilated and translated version of oscillations (wavelets) localized in both time and frequency. In wavelet analysis different mother wavelets can be used according to its feasibility to analyzed waveform. The main advantage of wavelet over the short time Fourier transform (STFT) is that it uses a variable-sized regions windowing technique, while STFT uses a fixed time window size for all frequencies. For nonstationary signals, STFT does not track the signal dynamics properly because of the fixed window width. Wavelet uses long time windows for low-frequencies and short time windows for high-frequencies. Figure 7.1 shows the time frequency resolution of Wavelet Transform (WT).

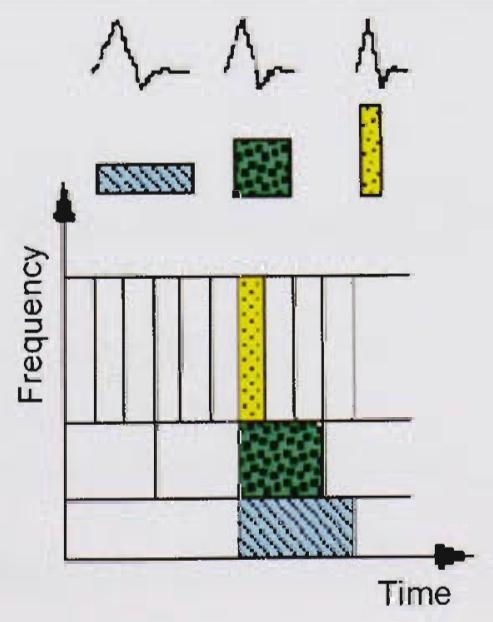

Fig.7.1 Time Frequency resolution of Wavelet Transform (WT) 
With the increase utilization of power electronics devices in the power system the harmonics and disturbances generated in the system can have a wide frequency bandwidth, from high-frequency transients and edges to slowly varying harmonic components. Hence, analysis only in the frequency or time domain alone is not sufficient to capture the signal features that are spread within a wide bandwidth. The wavelet transform provides a local representation (in both time and frequency) of a nonstationary signal. Therefore it is suitable for analyzing a signal where time and frequency resolution is needed, unlike FFT which gives a global representation of the signal.

\subsection{Continuous Wavelet Transforms (CWT)}

The continuous wavelet transform (CWT) was developed to overcome the short time Fourier transform frequency resolution problem. By using a family of basis functions, wavelets can be formulated to describe signals in a localized time and frequency format. A linear combination of these shifted and scaled basis functions can be applied to represent these functions in the space spanned by this wavelet family.

For a continuous time function $f(\mathrm{t})$ defined in finite energy signal space $L^{2}(R)$ that is

$$
\int_{-\infty}^{x}|f(t)|^{2} d t<\infty
$$

The wavelet transform $W_{x}(b, a)$ of a continuous-time signal $f(\mathrm{t})$ is defined as follows

$$
C W T_{\psi} x(a, n)=W_{x}(a, b)=|a|^{-0.5} \int_{-\infty}^{\infty} f(t) \psi^{*}\left(\frac{t-b}{a}\right) d t
$$

Where: $\psi(t)$ is the wavelet basis function and the asterisk denotes a complex conjugate $a, b \in R$ are the time shifting and the scaling parameters and $a \neq 0$. 
The scaling factor $|a|^{-0.5}$ is used in order to maintain the scaled wavelet energy at unity and therefore maintain the orthogonality property.

Thus, the wavelet transform is computed as the inner product of $f(\mathrm{t})$ and translated and scaled versions of a single function $\psi(t)$, the so-called mother wavelet.

\subsection{Choice of analyzing mother wavelet}

In wavelet applications, different wavelet mother functions have been suggested and utilized. The choice of analyzing mother wavelets plays a significant role in detecting and localizing various types of signals. The selection of the mother wavelet function depends on it's feasibility to the analysis requirements. However, the selection of an appropriate mother wavelet without knowing the type of analyzed disturbances is a formidable task.

A well chosen mother wavelet will result in that most wavelet coefficients are close to zero. The ability of the wavelet analysis to produce a large number of non-significant wavelet coefficients depends on the regularity of the analyzed waveform $f(\mathrm{t})$, and the number of vanishing moments and support size of $\psi(t)$.

Therefore, instead of creating algorithms to select appropriate wavelets, we chose the Daubechies wavelet family, which is one of the one of most suitable mother wavelets for power system relaying and power quality studies.

\subsection{Discrete Wavelet Transforms (DWT)}

\subsubsection{Multiresolution analysis}

As described in the previous section CWT is basically a type of convolution and convolution is a filtering process. The discrete wavelet transforms (DWT) can be computed by lowpass and highpass filtering of the discrete signal together with changes 
in its sampling rates. This process will decompose the signal into approximation version and detail version of the original signal. The approximation version results from convolution of the original signal with lowpass filter and the detailed results from convolution with highpass filter with downsampling by 2 . The original signal is the summation of its detailed and approximated version interpolated by a factor of two.

A multiresolution analysis of $L^{2}(R)$ is defined as a sequence of closed subspaces $V_{j}$ of $L^{2}(\mathrm{R}), \mathrm{j} \in \mathrm{Z}$, with the following properties [197-200]:

1. $V_{j} \subset V_{j+1}$

2. $f(t) \in V_{j} \Leftrightarrow v(2 t) \in V_{j+1}, j \in Z$

3. $f(t) \in V_{0} \Leftrightarrow v(t-k) \in V_{0}, k \in Z$

4. $\bigcup_{j=-\infty}^{+\infty} \mathrm{V}_{\mathrm{j}}$ is dense in $\mathrm{L}^{2}(\mathrm{R})$ and $\bigcap_{j=-\infty}^{+\infty} \mathrm{V}_{\mathrm{j}}=\{0\}$

5. A scaling function or father function $\varphi \in V_{o}$, with a non-vanishing integral, exists such that collection $\{\varphi\{t-k) \mid k \in Z\}$, forms a Riesz basis for $V_{o}$

A signal can be successively approximated by DWT with different scales known as multiresolution decomposition. Each step of the decomposition of the signal corresponds to a certain frequency resolution. The decomposition process can be repeated on subsequent approximations only and leave the details. The process will decompose the original signal into many lower-resolution frequency components. This is known as the wavelet decomposition tree.

For $\mathrm{j}$ level decomposition tree, there are $\mathrm{j}+1$ possible ways to decompose or encode the original signal. 


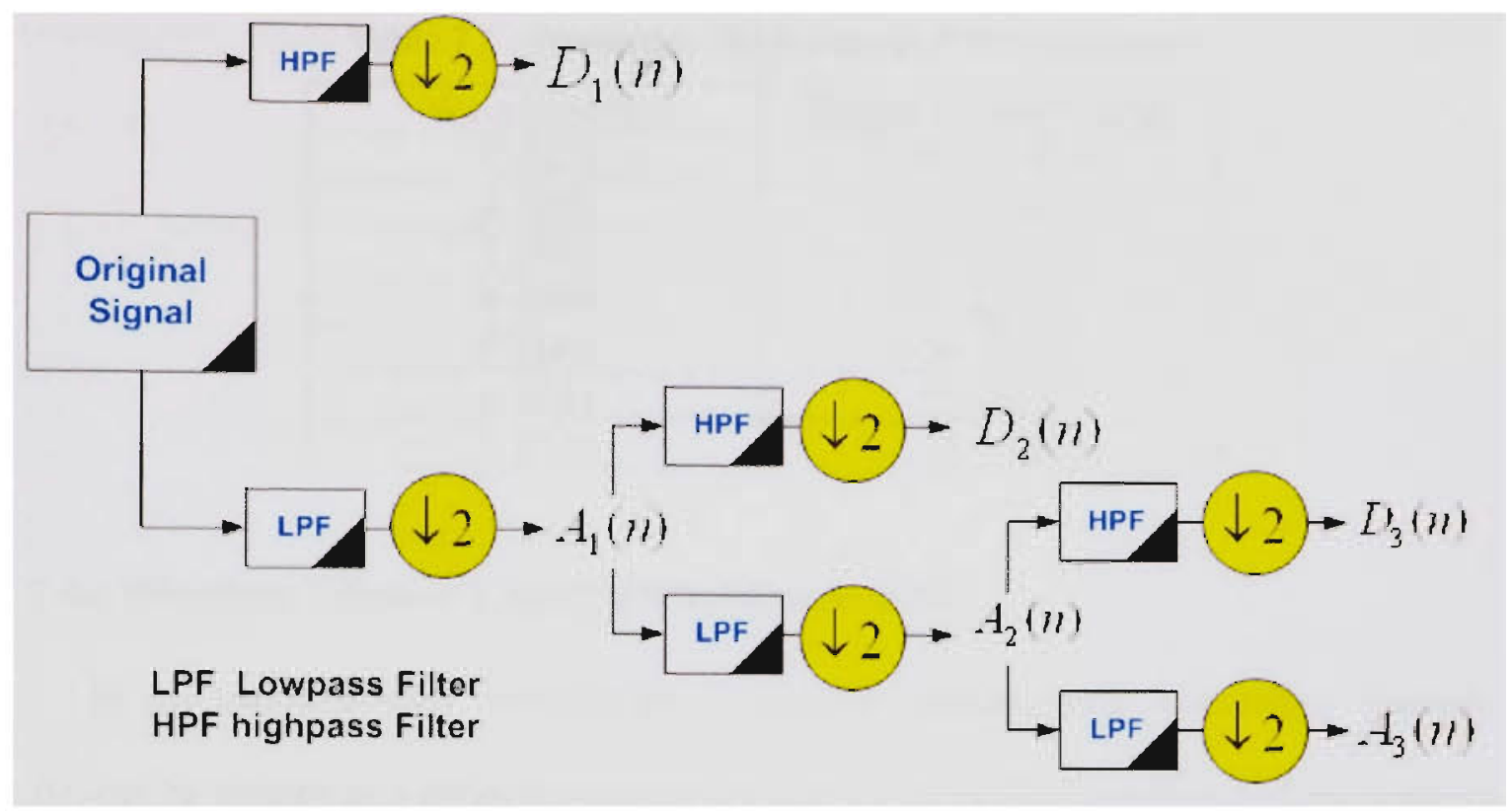

Fig.7.2 Three decomposition levels with successive filtering and downsampling by 2

Figure 7.2 shows a typical three levels wavelet decomposition tree. The series $A_{j}$ is known as the approximation coefficients, and the series $D_{j}$ is known as the detail coefficients at level $j$. Each of the wavelet scales or decomposition levels correspond to a frequency band given by [70]:

$$
f=2^{(n-j)}\left(f_{s} / 2^{n}\right)
$$

Where:

$$
\begin{aligned}
& f \text { higher frequency limit of the frequency band represented by level } j \\
& f_{s} \text { is the sampling frequency } \\
& 2^{n} \text { Number of data points in the input signal }
\end{aligned}
$$

Table 1 gives the frequency band information for seven levels of wavelet analysis for a sampling rate of $f_{s}=10 \mathrm{kHz}$. 
Table 7.1 Frequency Allocation in DWT Analysis

\begin{tabular}{|c|c|}
\hline Wavelet level & Frequency band range \\
\hline 1 (D1) & $2500-5000 \mathrm{~Hz}$ \\
\hline 2 (D2) & $1250-2500 \mathrm{~Hz}$ \\
\hline 3 (D3) & $625-1250 \mathrm{~Hz}$ \\
\hline 4 (D4) & $312-625 \mathrm{~Hz}$ \\
\hline 5 (D5) & $156-312 \mathrm{~Hz}$ \\
\hline 6 (D6) & $39-78 \mathrm{~Hz}$ \\
\hline 7 (A6) & $20-39 \mathrm{~Hz}$ \\
\hline
\end{tabular}

\subsubsection{Waveform Effective Value Representation in DWT}

In the multiresolution formulation of discrete wavelets, any real valued function $i(t)$ can be written as a series expansion in terms of the scaling function and wavelets as follows:

$$
i(t)=\sum_{n=0}^{2^{N}-1} B_{J_{0}}(n) \phi_{J_{n, n}, n}(t)+\sum_{j=J_{0}}^{J-1} \sum_{n=0}^{2^{N-1}-1} B_{j}(n) \psi_{j, n}(t)
$$

The RMS value of this waveform with period $T=2^{N}$ can be expressed in terms of wavelet at a certain level $j$ as follows [70-76]:

$$
i_{r m s}=\sqrt{\frac{1}{T} \int_{0}^{T}\left[\left(\sum_{n=0}^{2^{N}-1} B_{J_{11}}(n) \phi_{J_{0}, n}(t)+\sum_{j=J_{0}}^{J-1} \sum_{n=0}^{2^{N-1}-1} B_{j}(n) \psi_{j, n}(t)\right)\right]^{2} d t} .
$$

Where: $\psi_{j, n}(t)$ is the bandpass wavelet function,

$$
\begin{aligned}
& \phi_{j, n}(t) \text { is the lowpass scaling function, } \\
& B_{0}(n)=\left\langle i(t), \phi_{J_{1}, n}(t)\right\rangle \text {, is the scaling function coefficients, } \\
& B_{j}(n)=\left\langle i(t), \psi_{j, n}(t)\right\rangle, \text { is the DWT coefficients of } i(t) \text { at level } j .
\end{aligned}
$$

The node $j=J_{0}$ is the lowest frequency band of the original waveform. The nodes $j>J_{0}$ include the waveforms of higher frequency order bands. 
Since the wavelet basis functions are orthogonal, the following properties should be fulfilled:

$$
\begin{aligned}
& \int \psi_{j, k}^{P}(t) d t=0 \quad \mathrm{p}=\text { integer } \\
& \int \psi_{j, k}^{P}(t) \psi_{j, k}^{q}(t)=\left\{\begin{array}{cc}
1 & \mathrm{p}=\mathrm{q} \\
0 & p \neq q
\end{array}\right. \\
& \int\left(\phi_{j, k}(t)\right)^{2}=1 \\
& \int \phi_{j, k}(t) \psi_{j, k}^{p}(t)=0 \quad \mathrm{p} \neq 0
\end{aligned}
$$

Using the wavelet scaling function and the wavelet basis orthogonality property, equation (7-6) becomes:

$$
i_{r m s}=\sqrt{\frac{1}{2^{N}} \sum_{j=0}^{j-1} \sum_{n=0}^{2^{N-j}-1}\left(B_{j}(n)\right)^{2}}=\sqrt{\sum_{J=0}^{j-1}\left(i_{j}\right)^{2}} .
$$

Where, $i_{j}$ is the RMS value of frequency band $j$

\subsection{Wavelet Packet Transform Algorithm (WPT)}

\subsubsection{Theory}

The wavelet packet transform (WPT) method is a generalization of wavelet decomposition. In wavelet packet analysis, the original signal is decompose into detailed version and approximated version. All the subsequent details and approximations version are also split into details and approximations version. For $\mathrm{j}$ level decomposition level there are more than $2^{2^{j-1}}$ different ways to decompose the original signal.

The DWT logarithmic frequency resolution is not appropriate for some application like power quality studies which required equal frequency bands. Because the resulted frequency bands from DWT do not have the same frequency width. Wavelet Packets 
allow for the decomposition of the waveforms into equal frequency width bands. Figure 7.3 shows a two level WPT decomposition levels.

In Fig. 7.3, each subspace, also referred to as a node, is indexed by its depth and the number of subspaces below it at the same depth. The original signal is designated depth zero.

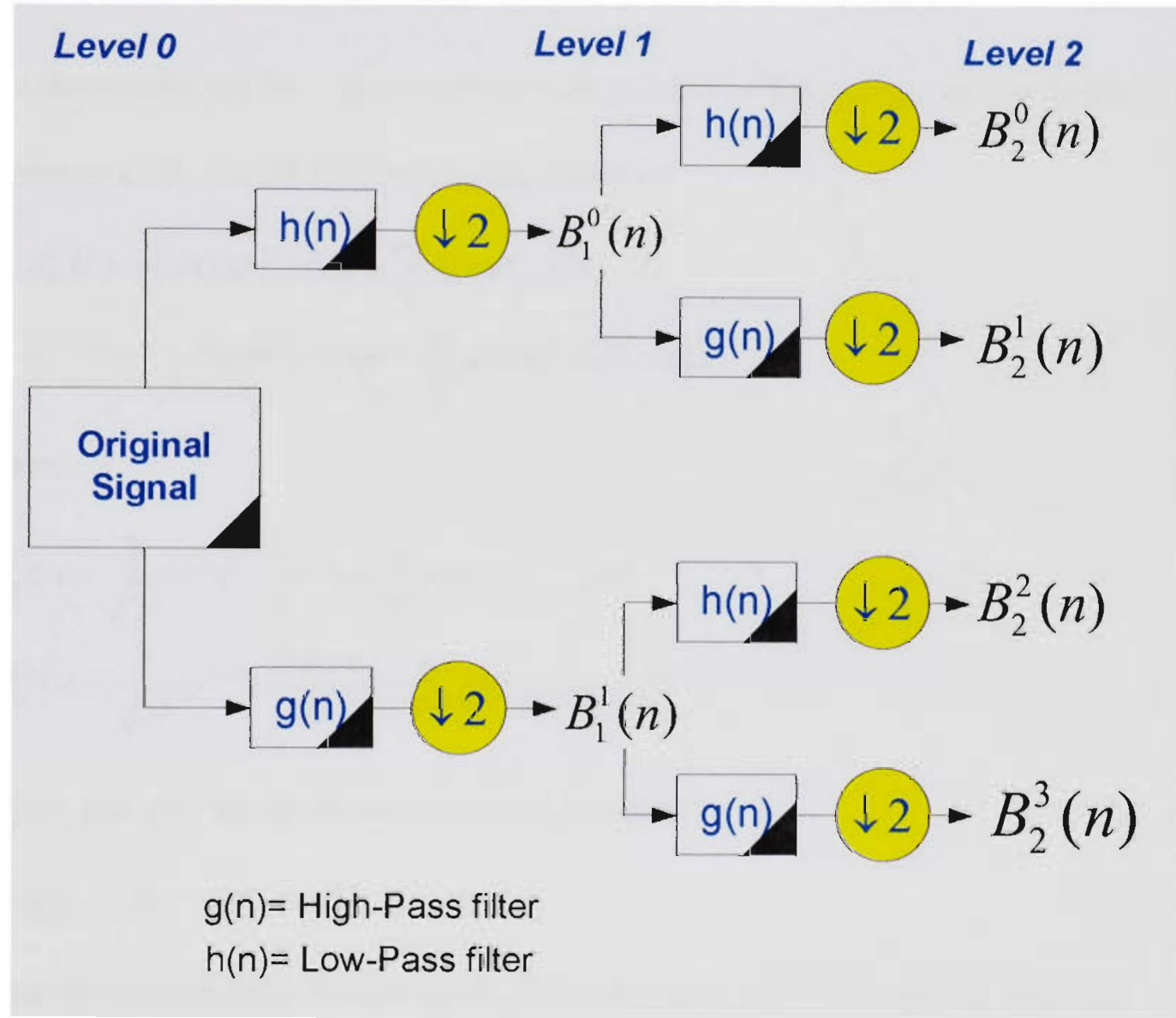

Fig.7.3 WPT two decomposition levels with successive filtering and downsampling by 2

The wavelet packet reconstruction scheme is achieved by upsampling, and filtering with appropriate filters and adding coefficients resulting from the decomposition process. Let $\phi(t)$ and $\psi(\mathrm{t})$ be the scaling function and the corresponding mother wavelet function in the conventional DWT and define $\psi^{\circ}(t)=\phi(t)$, and $\psi^{\prime}(t)=\psi(t)$, 
For $2^{N}$ sampled waveform the wavelet transform coefficients (WTCs) of a given function $\mathrm{B}(\mathrm{t})$ at the level $j$ and $k^{t h}$ point can be evaluated by convolving the sequence $d_{j-1}^{i}(k)$ with a low pass filter (LPF), and then downsampling by a factor of two. In the same manner, coefficients (WTCs) of a given function $i(t)$ at the $(2 i+1)^{t h}$ node can be evaluated obtained by convolving the sequence $d_{j-1}^{i}(k)$ with a highpass filter (HPF), and then downsampling by a factor of two. The number of frequency bands at level $j$ is $2^{j}$. Mathematically, the (WTCs) can be expressed as:

$$
\begin{aligned}
& d_{j}^{2 i}(k)=\int B(t) \psi_{j, k}^{2 i}(t) d t=\sum_{n} h(n) d_{j-1}^{i}(2 k-n) \\
& d_{j}^{2 i+1}(k)=\int B(t) \psi_{j, k}^{2 i+1}(t) d t=\sum_{n} g(n) d_{j-1}^{i}(2 k-n)
\end{aligned}
$$

Where:

$$
\begin{aligned}
& \psi_{j, k}^{2 i}(t)=\frac{1}{\sqrt{2^{j}}} \psi^{2 i}\left(\frac{2^{j} k-t}{2^{j}}\right)=\sum_{n} h(n) \psi_{j-1,2 k-n}^{i}(t) \\
& \psi_{j, k}^{2 i+1}(t)=\frac{1}{\sqrt{2^{j}}} \psi^{2 i+1}\left(\frac{2^{j} k-t}{2^{j}}\right)=\sum_{n} g(n) \psi_{j-1,2 k-n}^{i}(t)
\end{aligned}
$$

$\psi_{j, k}^{2 i}(t)$ and $\psi_{j, k}^{2 i+1}(t)$ are the wavelet basis functions

$$
j=0,1, \ldots, \mathrm{N} \quad \text { and } i=0,1, \ldots \ldots, 2^{j-1}-1
$$

Since the wavelet basis is orthogonal, the following properties should be fulfilled:

$$
\begin{aligned}
& \int \psi_{j, k}^{P}(t) d t=0 \quad \mathrm{p}=\text { integer } \\
& \int \psi_{j, k}^{P}(t) \psi_{j, k}^{q}(t)=\left\{\begin{array}{cc}
1 & \mathrm{p}=\mathrm{q} \\
0 & p \neq q
\end{array}\right. \\
& \int\left(\phi_{j, k}(t)\right)^{2}=1 \\
& \int \phi_{j, k}(t) \psi_{j, k}^{p}(t)=0 \quad \mathrm{p} \neq 0
\end{aligned}
$$


7.3.2 WPT Representation of the waveform indices

In DWT the signal $i(t)$ can be represented in terms of shifted and dilated versions of a bandpass wavelet function $\psi_{j, k}^{i}(t)$ and lowpass scaling function $\phi_{j . k}(t)$ as follows:

$$
i(t)=\sum_{n=0}^{2^{N-1}-1} B_{j}^{0}(n) \phi_{j, k}(t)+\sum_{i=1}^{2^{\prime}-1} \sum_{n=0}^{2^{N-1}-1} B_{j}^{i}(n) \psi_{j, k}^{i}(t)
$$

Where:

$B_{j}^{0}(n)=\left\langle i(t), \phi_{j, k}(t)\right\rangle=\int i(t) \phi_{j, k}(t) d t$, is the scaling function coefficients.

$B_{j}^{i}(n)=\left\langle i(t), \psi_{j, k}^{i}(t)\right\rangle$, is the DWT coefficients of $i(t)$ at node $i(i>0)$ and level $j$.

The node $i=0$ is the lowest frequency band of the original waveform. The nodes $i>0$ include the waveforms of higher frequency order bands.

The RMS of a waveform with period $\mathrm{T}=2^{N}$ can be expressed in terms of wavelet at a certain level $j$ as follows:

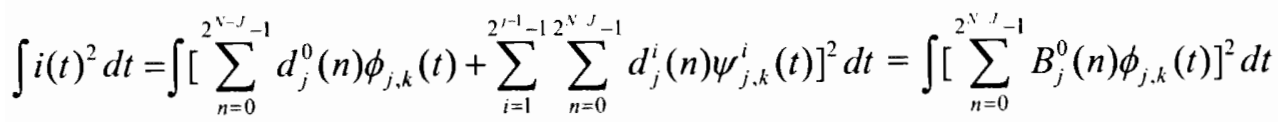

$$
\begin{aligned}
& \left.+\int\left[\sum_{i=1}^{2^{\prime}-1} \sum_{n=0}^{2^{x j}-1} B_{j}^{i}(n) \psi_{j, k}^{i}(t)\right]^{2} d t\right]^{2}+2 \int\left(\sum_{i=1}^{2^{i}-1} \sum_{n=0}^{2^{*}, t} B_{j}^{0}(n) B_{j}^{i}(n) \phi_{j, k}(t) \psi_{j, k}^{i}(t)\right) \\
& =\left[\sum_{n=0}^{2^{N-J}-1}\left(B_{j}^{0}(n)\right)^{2} \int\left[\phi_{j, k}(t)\right]^{2} d t+\sum_{i=1}^{2^{j-1}-1} \sum_{n=0}^{N-J}\left(B_{j}^{i}(n)\right)^{2} \int\left[\psi_{j, k}^{i}(t)\right]^{2} d t\right. \\
& +2\left(\sum_{i=1}^{2^{\prime} 1-1} \sum_{n=0}^{N-2^{-1}-1} B_{j}^{0}(n) B_{j}^{i}(n) \int \phi_{j, k}(t) \psi_{j, k}^{i}(t) d t\right)
\end{aligned}
$$

The RMS of the waveform can now found by dividing by the wave period $\mathrm{T}$ and take the root square of Eq. (7-11).

$$
i_{r m s}=\sqrt{\frac{1}{T} \int_{0}^{T}\left[\left(\sum_{n=0}^{2^{N}-1} B_{j}^{0}(n) \phi_{j, k}(t)+\sum_{i=1}^{2^{\prime}-12^{N} \sum_{n=0}^{\prime}-1} B_{j}^{i}(n) \psi_{j, k}^{i}(t)\right)\right]^{2} d t} .
$$


Using the wavelet the scaling function and the wavelet basis orthogonality property, Eq. (7-12) becomes:

$$
i_{r m s}=\sqrt{\frac{1}{2^{N}} \sum_{i=0}^{2^{\prime}-1} \sum_{n=0}^{2^{N-i}-1}\left(B_{j}^{i}(n)\right)^{2}}=\sqrt{\sum_{i=0}^{2^{i}-1}\left(i_{j}^{i}\right)^{2}} .
$$

Where, $i_{j}^{i}$ is the RMS value of frequency band $j$ at node $i$

The total harmonic band distortion (THBD) is defined by the ratio of the RMS value of the higher harmonic bands at $\mathrm{i}>0$ (i.e. excluding the lowest band) to the total RMS value of the distorted waveform. The THBD for the signal $i(t)$ is given by

$$
\mathrm{THBD}=\frac{1}{i_{r m s}} \sqrt{\sum_{i=1}^{2^{i}-1}\left(i_{j}^{i}\right)^{2}}
$$

This technique can be used to quantify different types of power quality disturbances and faults. Wavelet based techniques has high ability to extract the different harmonic components disregarding the length of their occurrence in time. The variable windowing feature will make wavelet capable of handling nonstationary signals. 


\section{Chapter 8: Classification and Characterization of Power System Events Using}

\section{Wavelets}

\subsection{Introduction}

This chapter deals with classification of power system events using wavelet based algorithm. This includes application of DWT and WPT described in chapter 7 to classify power system events. The main advantage of wavelet over the short time Fourier transform (STFT) is that it uses a variable-sized regions windowing technique, while STFT uses a fixed time window size for all frequencies and this imposes limitations for the analysis of low-frequency and high-frequency nonstationary signals at the same time. This chapter is divided into two sections the first section deals with the application of DWT as a feature extraction for the fault signal waveforms, and the second section deals with the application of WPT to power quality problems.

\subsection{Application of DWT as feature extractor for fault signals}

Harmonics and discontinuities generated by power system faults can have a wide frequency bandwidth, from high-frequency transients and edges to slowly varying harmonic components. Also the increase utilization of power electronics devices in the power system gives rise to harmonics in the system. Hence, analysis only in the frequency or time domain alone is not sufficient to capture features that are spread within a wide bandwidth. The wavelet transform provides a local representation (in both time and frequency), of faults nonstationary signals. Therefore, it is suitable for analyzing a signal where time and frequency resolution is needed, unlike FFT which gives a global presentation of the signal. 
In this section we will utilize DWT to classify internal faults in three phase transformer and three phase induction motor.

\subsubsection{Characterization of three phase transformers internal faults using DWT}

FEM was used in chapter 4 to model transformer with internal faults. Then DWT was used to extract the characteristics of the internal faults. The three phase currents, the DC side load current and the neutral current waveforms were then analyzed using DWT for different faults and healthy condition. The DWT feature extraction algorithm details were discussed in Chapter 7. A four level decomposition with $\mathrm{db} 20$ as the mother wavelet was selected to perform our study. Wavelet $\mathrm{db} 20$ is an orthogonal mother wavelet from Daubechies family with compact support and highest number of vanishing moments.

Fig. 8.1 through 8-4 shows the Wavelet transform coefficients (WTCs) for phase c primary, phase b primary, phase a primary and the dc side currents respectively for turnto-ground fault with 24 shorted turns on the primary side.

The event appears to be localized in the approximation A4, detail D4, and detail D1. It can be seen that the details DWT coefficients at the time of the fault occurrence are much higher than their values after or before this time. The details D1-D4 of the three phase primary currents contain a sharp spike at fault occurrence. The fourth approximation (A4) which indicates the low frequency content of the fault signal shows a high fluctuation in the current with magnitude increase after the fault occurrence for phase a current and phase c current and magnitude decrease for phase b current. The first detail (D1) RMS which indicates the high frequency content of the fault signal shows a high increase (up to $100 \%$ increase) after the fault occurrence for phase a current and phase $\mathrm{c}$ current and no change for phase $\mathrm{b}$ current. 


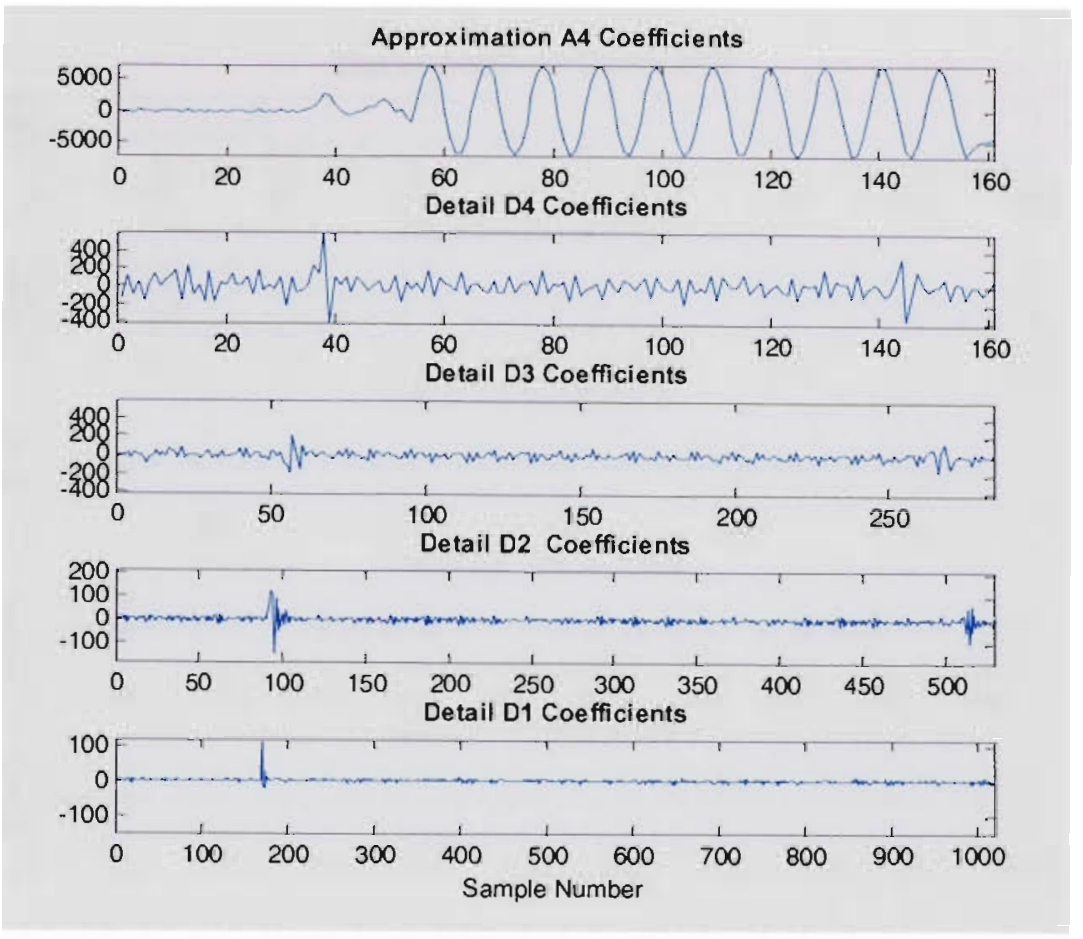

Fig.8.1. WTCs of phase c primary current for primary phase $\mathrm{c} 24$ shorted turn-to-ground

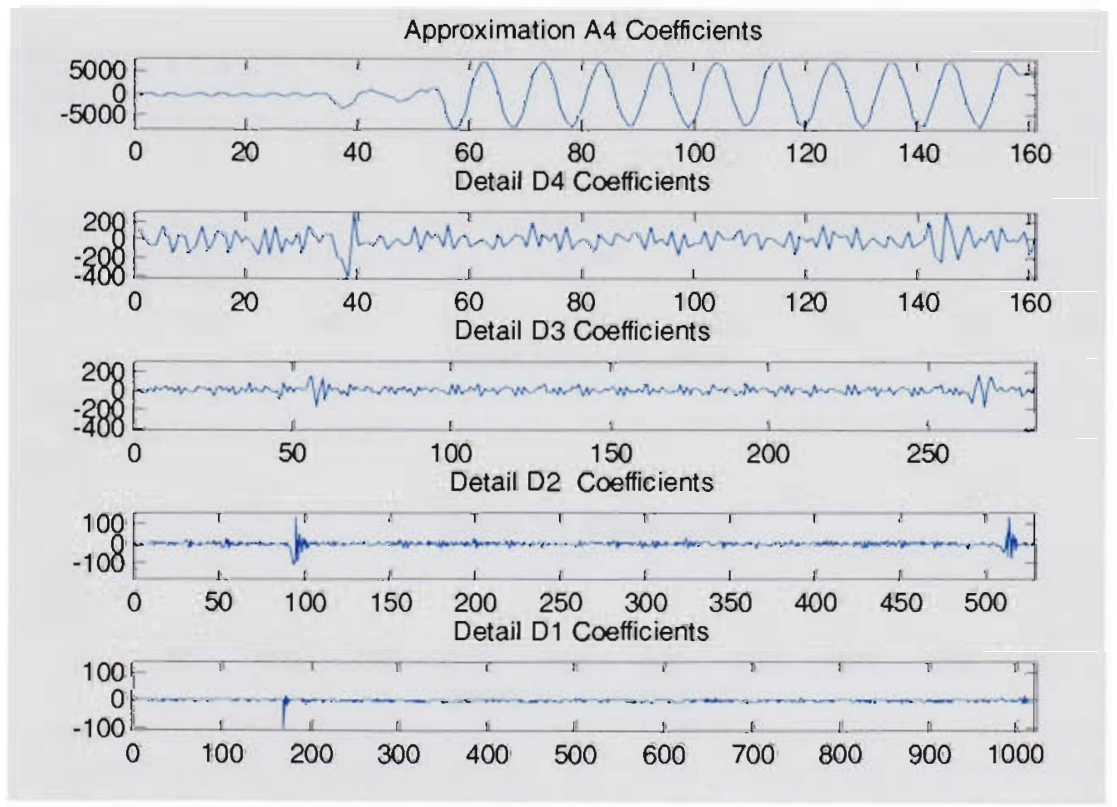

Fig. 8.2. WTCs of phase a primary current for primary phase c 24 shorted turn-to-ground 


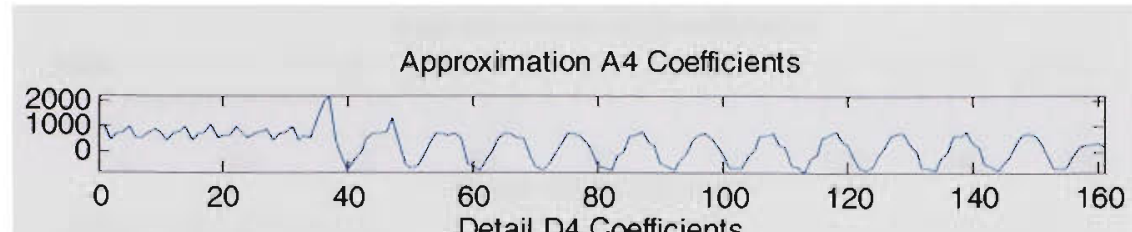

Detail D4 Coefficients
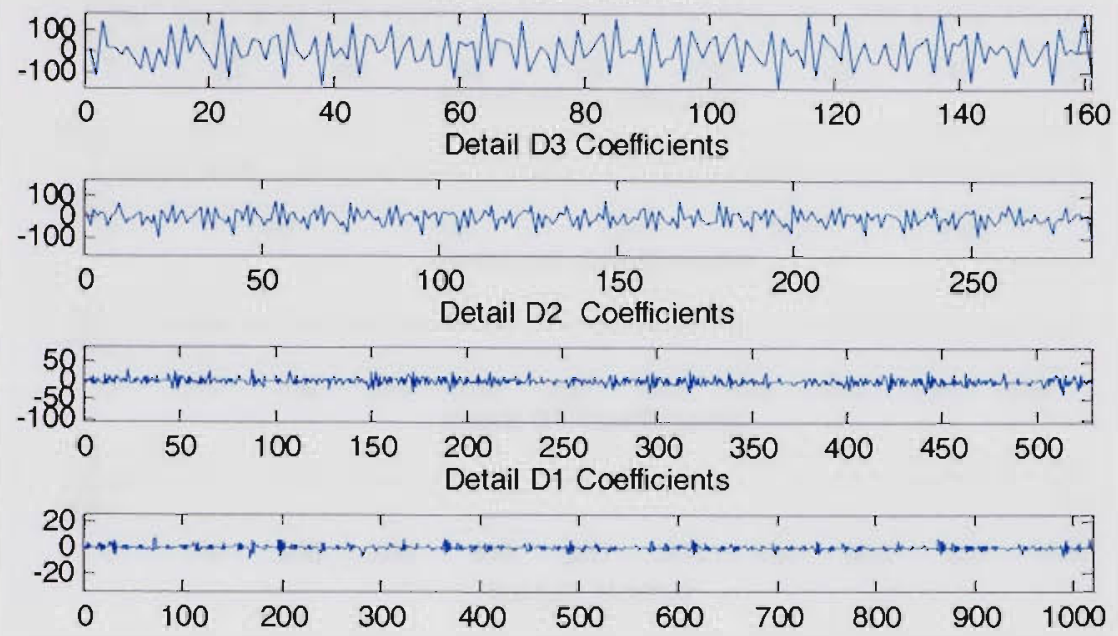

Fig.8.3. WTCs of phase b primary current for primary phase $\mathrm{c} 24$ shorted turn-to-ground

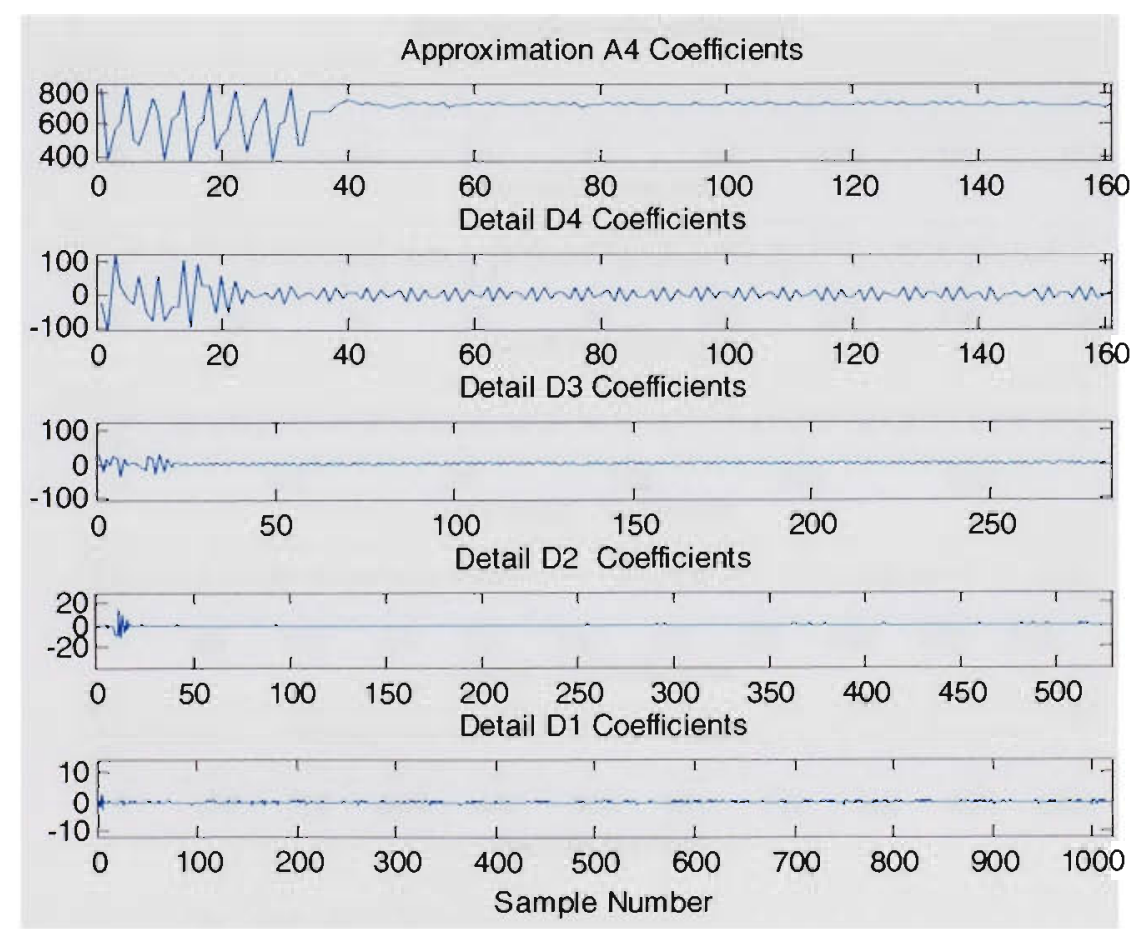

Fig.8.4. WTCs of the DC side current for primary phase c 24 shorted turn-to-ground 


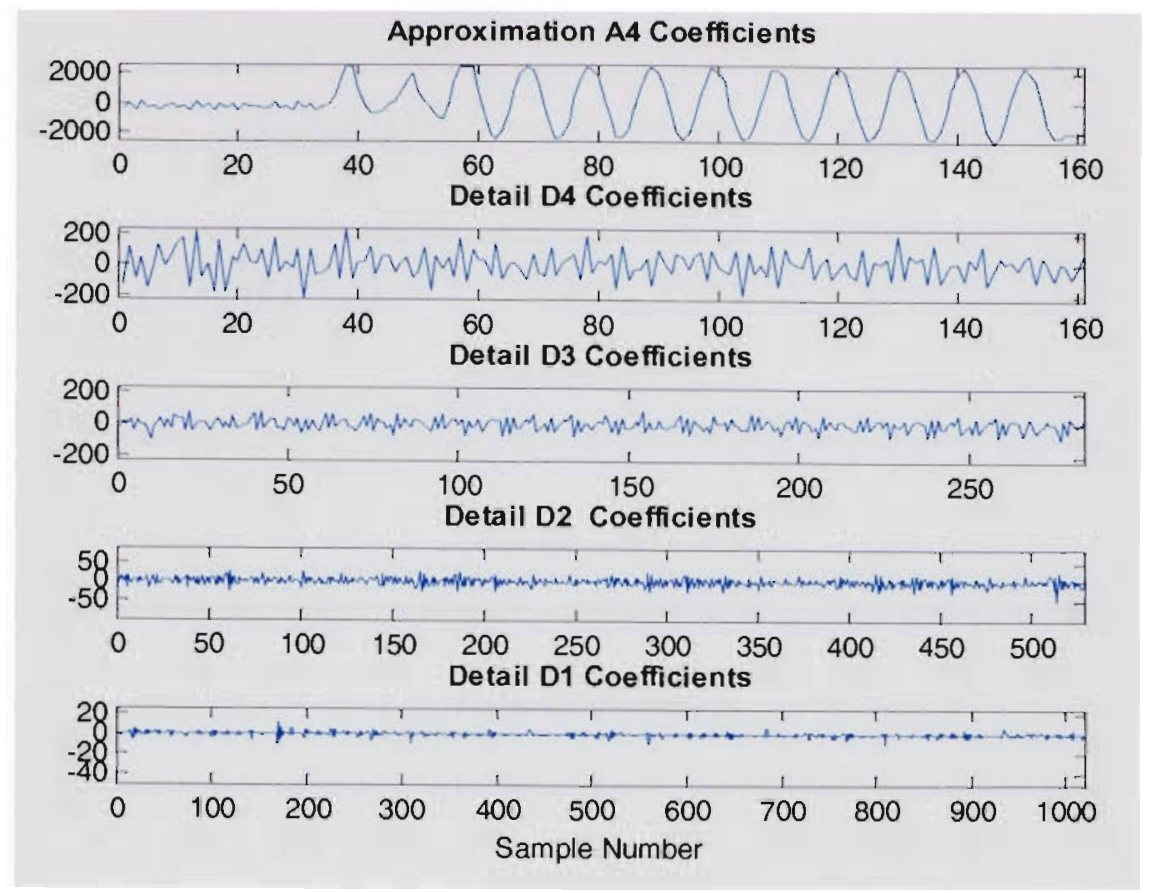

Fig.8.5. WTCs of primary phase c current for turn-to-turn fault on the primary of phase $\mathrm{c}$

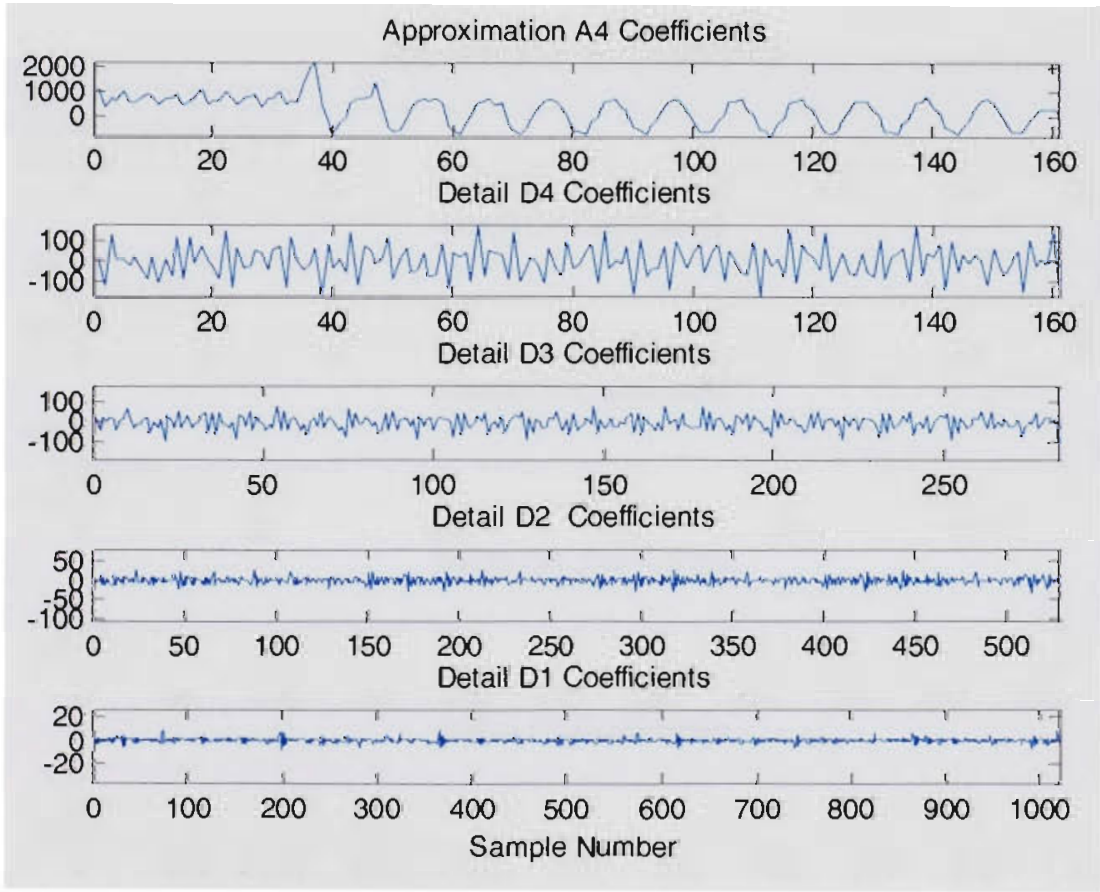

Fig.8.6. WTCs of primary phase b current for turn-to-turn fault on the primary of phase c 


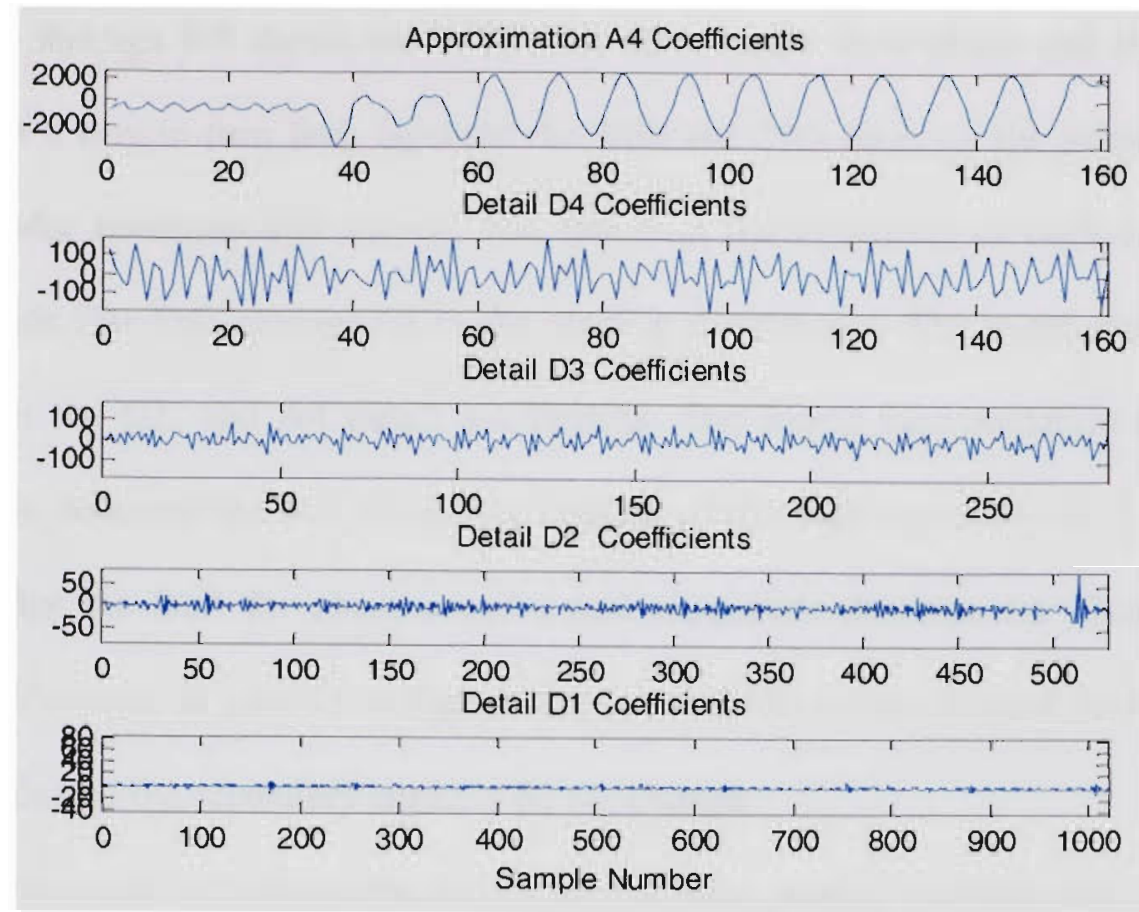

Fig.8.7. WTCs of primary phase $\mathrm{b}$ current for turn-to-turn fault on the primary of phase $\mathrm{c}$

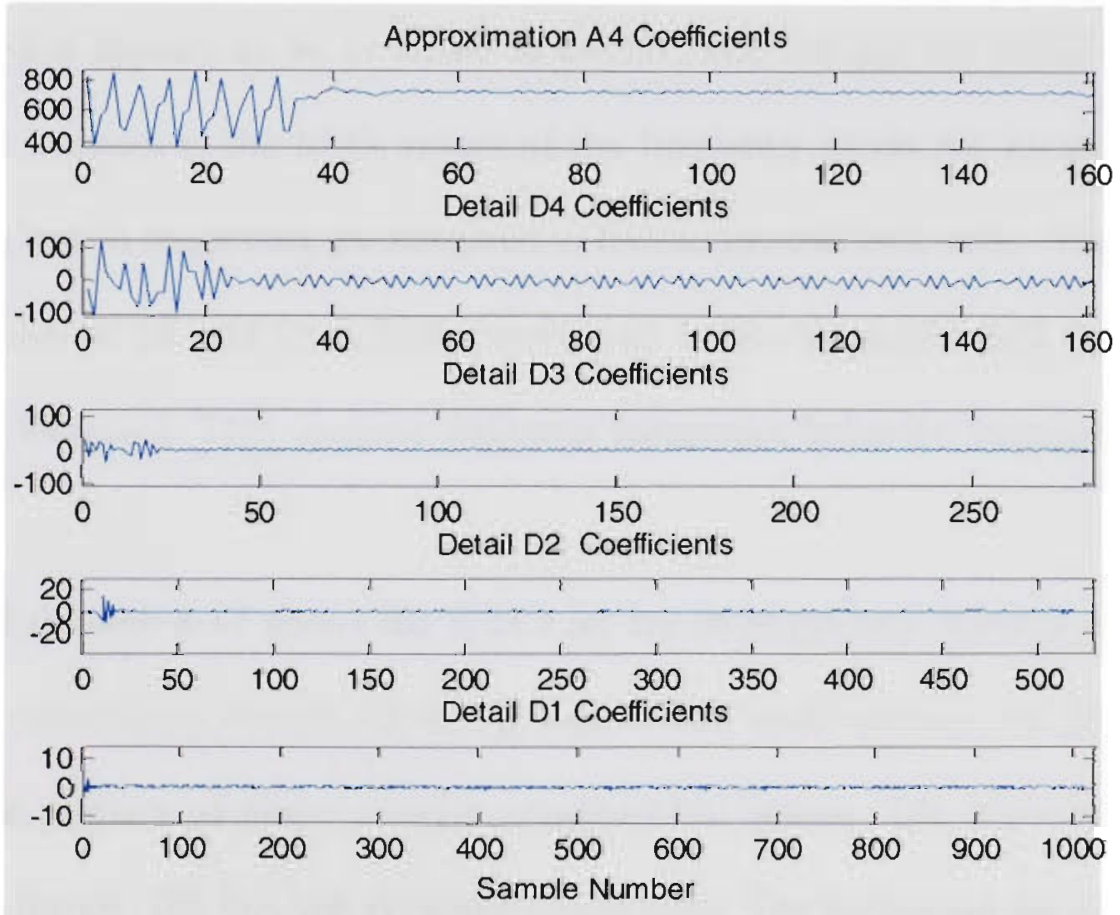

Fig. 8.8. WTCs of the DC side current for turn-to-turn fault on the primary of phase c 
Fig 8.5 through $8-8$ shows the WTCs for the primary three phase and the DC side currents for a turn-to-turn fault between the 20th and 29th turns on the primary side of phase $\mathrm{c}$ under nonsinusoidal source. The spikes at the beginning of each of the scale detail signals (D1-D4) correspond to the starting disturbance. The event appears to be localized in the D3, and A4 detail coefficients. The fourth approximation (A4) RMS value which indicates the low frequency content of the fault signal shows a magnitude increase after the fault for phase a and $\mathrm{c}$ and magnitude decrease for phase $\mathrm{b}$. This magnitude decrease in phase $\mathrm{b}$ is higher compared to the turn-to-ground fault case. The DC load side and the secondary currents do not change.

Fig 8.9 through 8-12 shows the WTCs for the three primary currents and the DC side for arcing turn-to-ground fault on phase c primary under nonsinusoidal source. In this fault, the event appears to be localized in the D1, D3, D4 and A4 detail coefficients. There is an increase in the RMS values of the frequency bands A4 for phase a and c currents but less in magnitude as compared to turn-to-ground fault only. The increase in the RMS value of Dl and D4 is higher compared to turn-to-ground fault only (between $75 \%-150 \%$ increase). This increase indicates harmonics behavior increase due to the arcing.

Fig 8.13 through $8-17$ shows the WTCs for the three primary currents, the DC side, and phase a secondary current for arcing turn-to-turn fault between the 20th and 29th turns on the primary of phase $c$ under nonsinusoidal source. The event appears to be localized in the D1, D2, D3, and A4 detail coefficients. The increase in the RMS value of D1, D2, and D4 is higher compared to turn-to-turn fault only. This increase indicates harmonics behavior increase due to the non linear resistance of the arcing. 


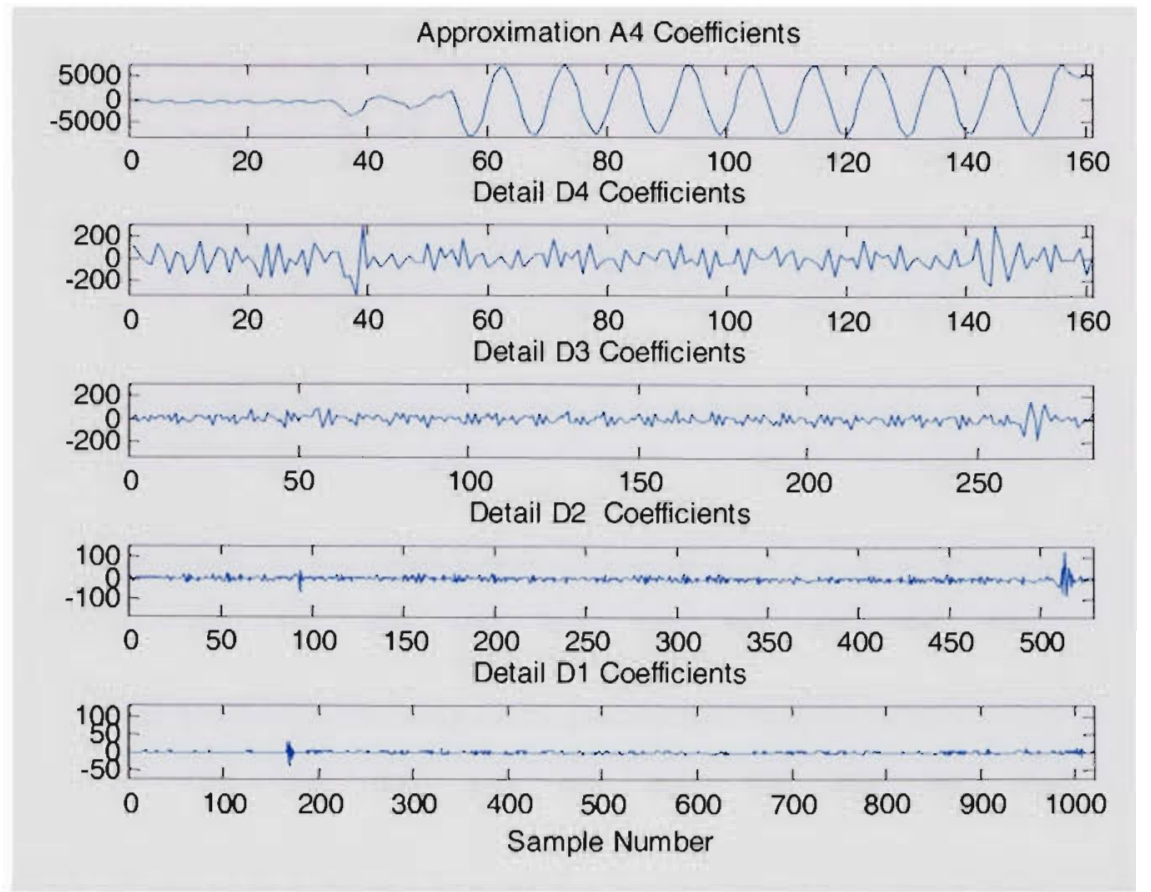

Fig.8.9. WTCs of phase c primary current for arcing turn-to-ground fault on the primary of phase $\mathrm{c}$

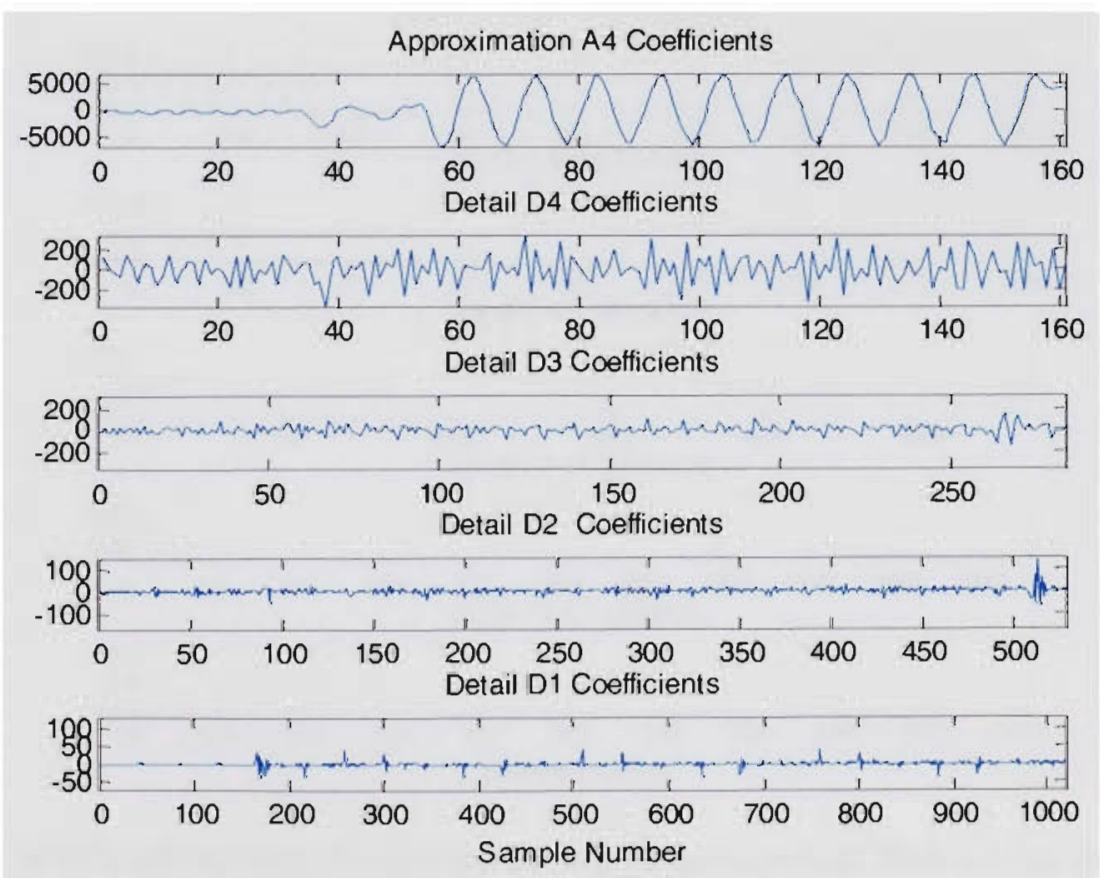

Fig.8.10. WTCs of phase a primary current for arcing turn-to-ground fault on primary of phase $\mathrm{c}$ 


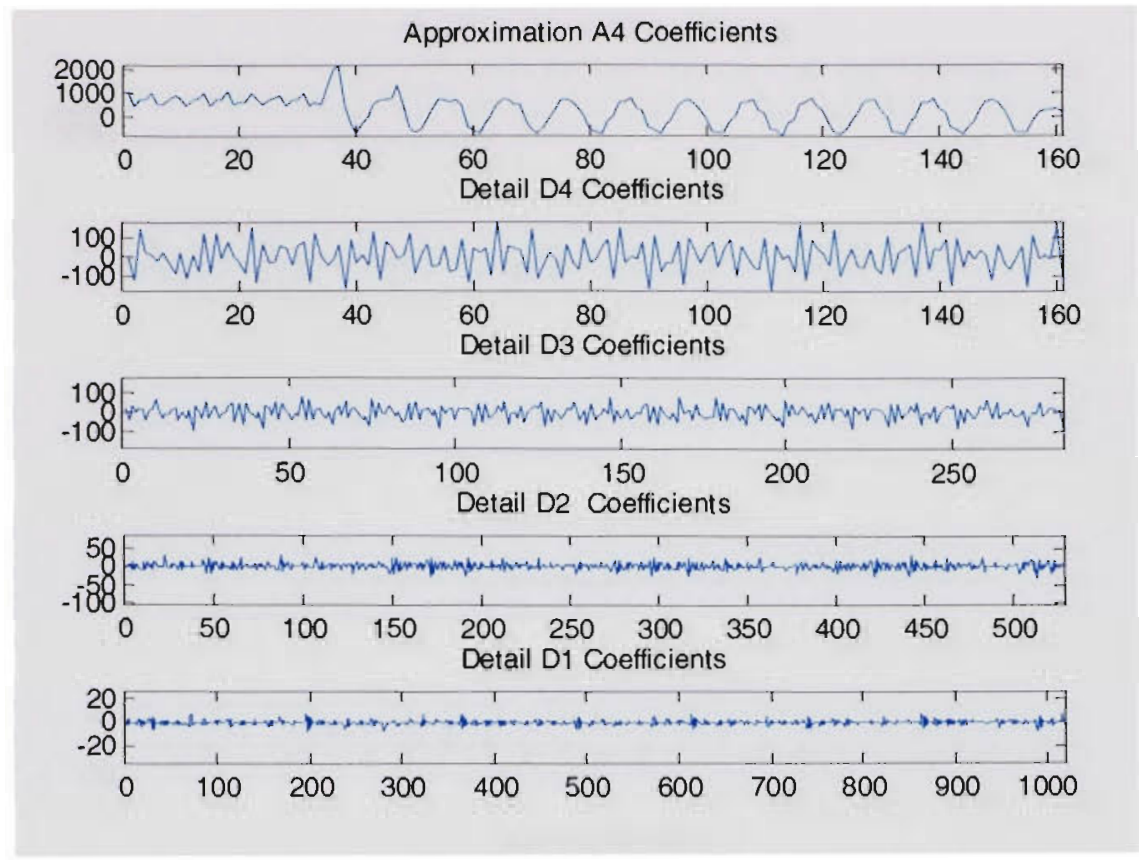

Fig. 8.11. WTCs of phase b primary current for arcing turn-to-ground fault on the primary of phase $c$

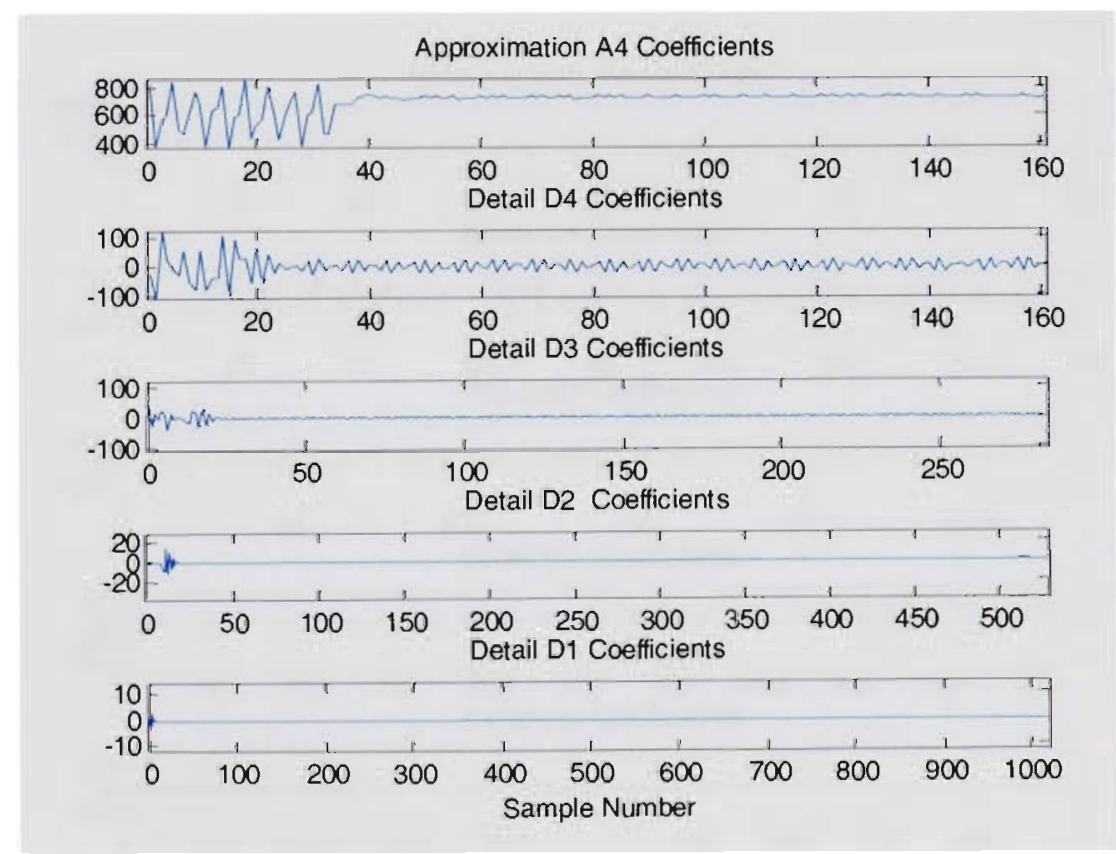

Fig.8.12. WTCs of DC side current for arcing turn-to-ground fault on the primary of phase $\mathrm{c}$ 


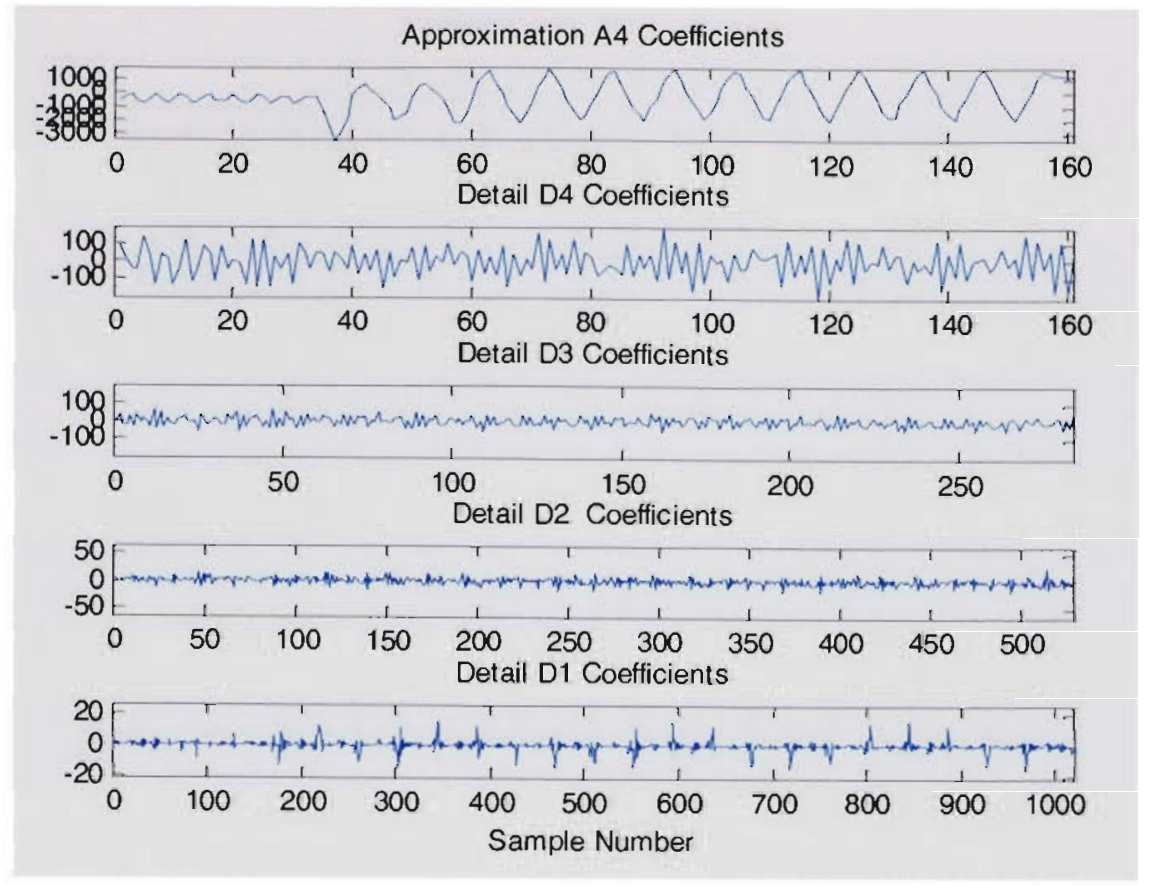

Fig.8.13. WTCs of phase a primary current for arcing turn-to-turn fault the primary of phase $\mathrm{c}$

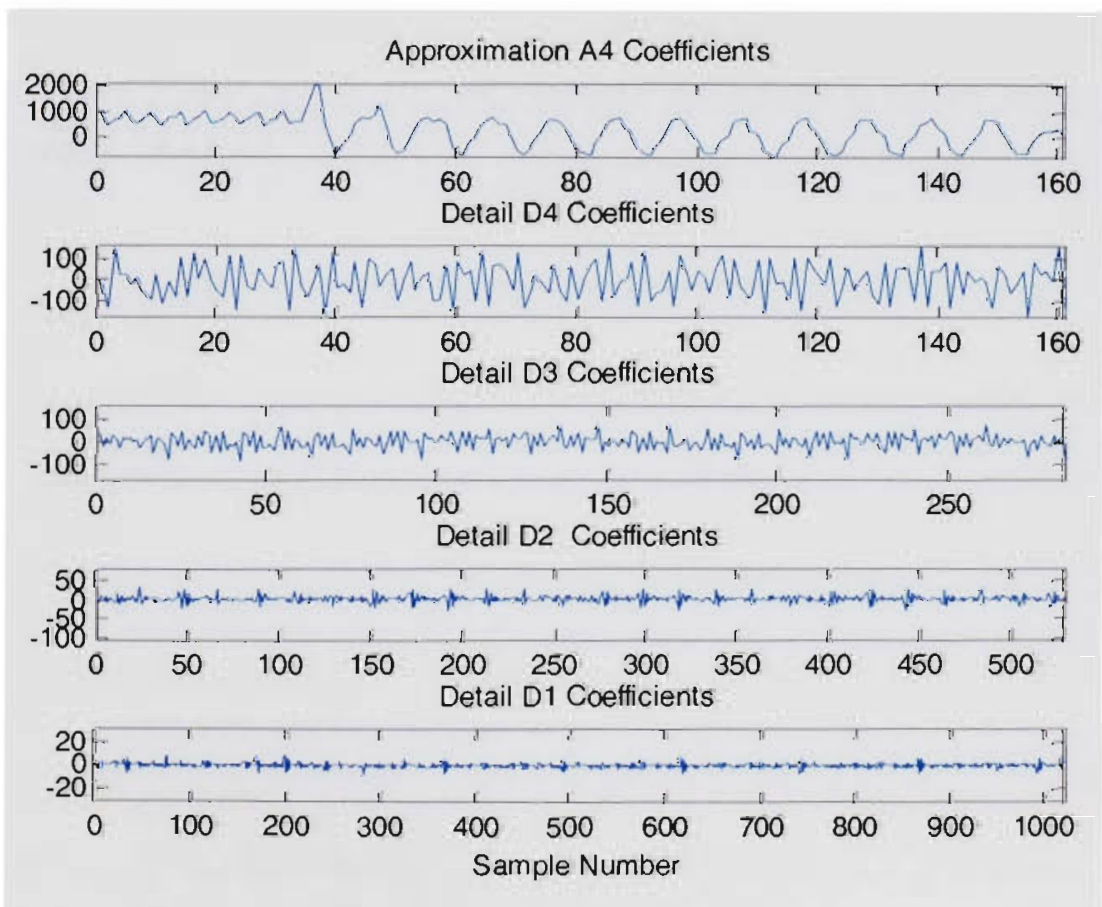

Fig.8.14. WTCs of phase b primary current for arcing turn-to-turn fault on the primary of phase c 


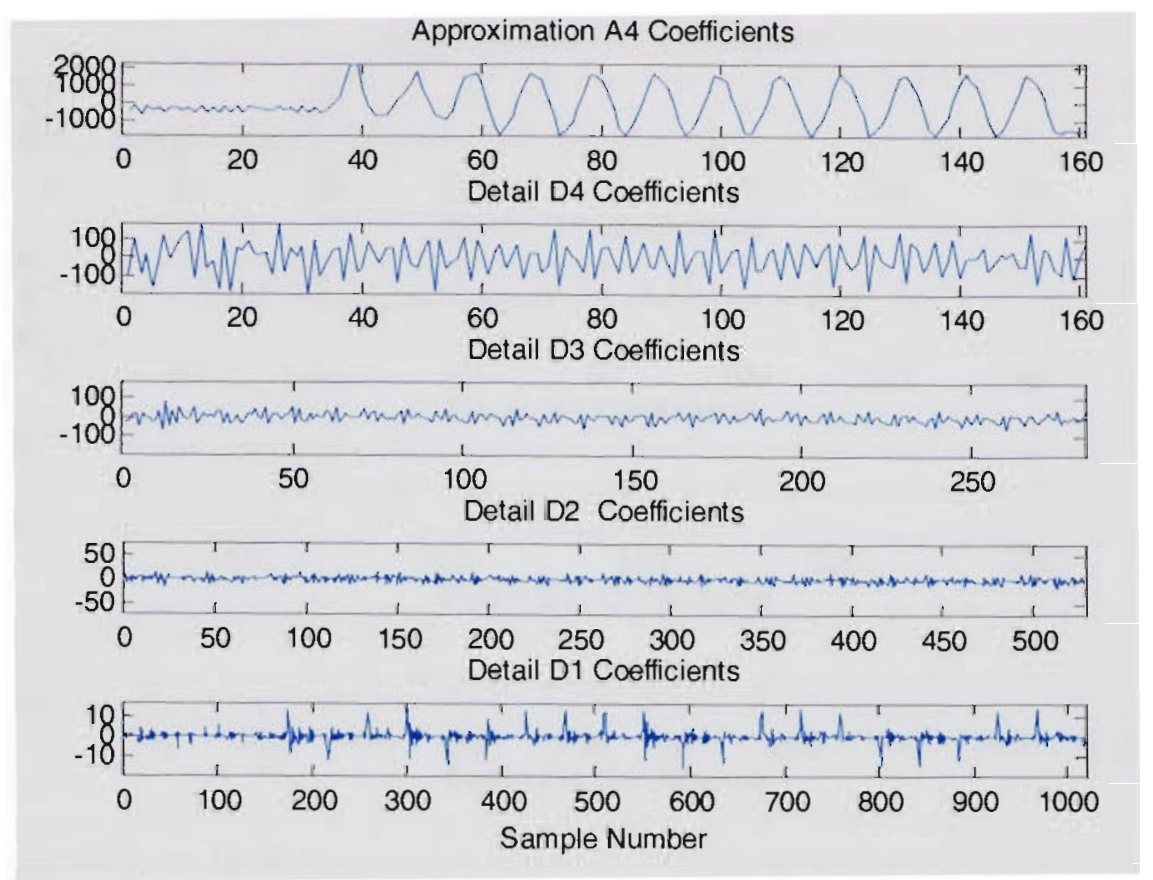

Fig.8.15. WTCs of phase c primary current for arcing turn-to-turn fault on the primary of phase $c$

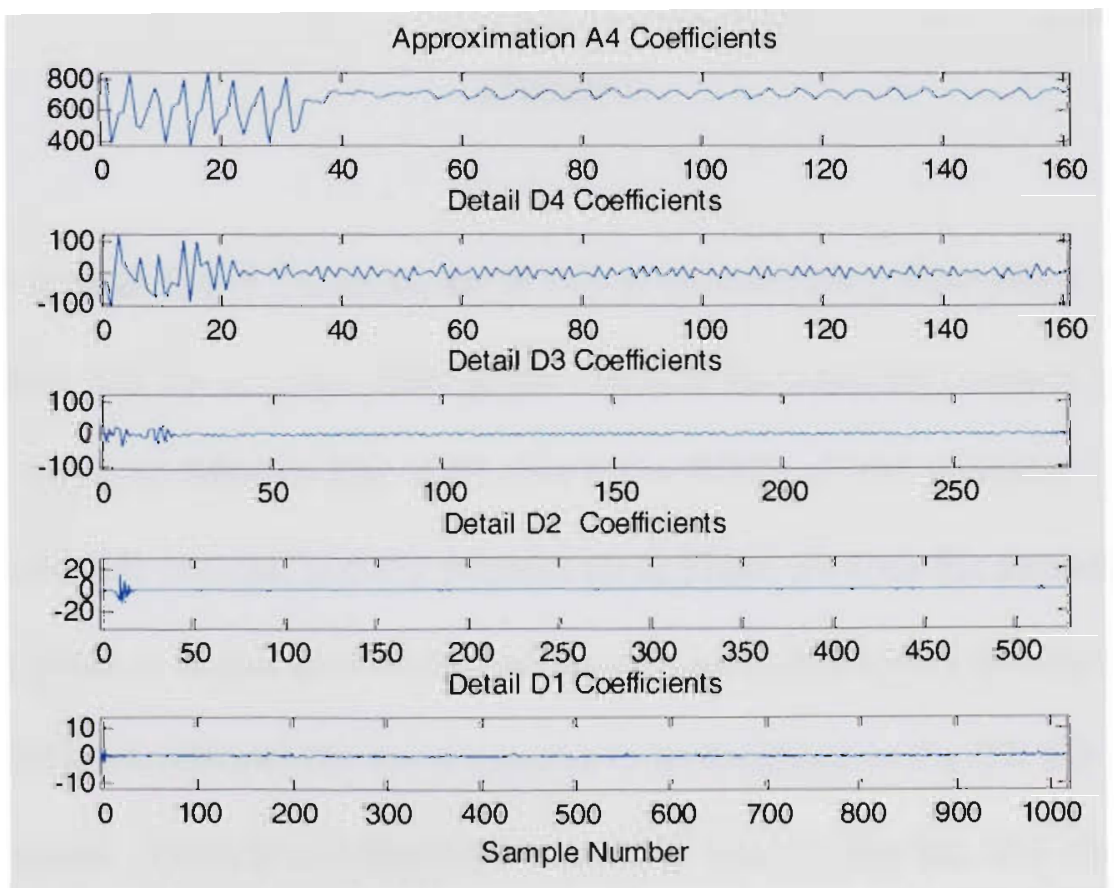

Fig.8.16. WTCs of DC side current for arcing turn-to-turn fault on the primary of phase c 


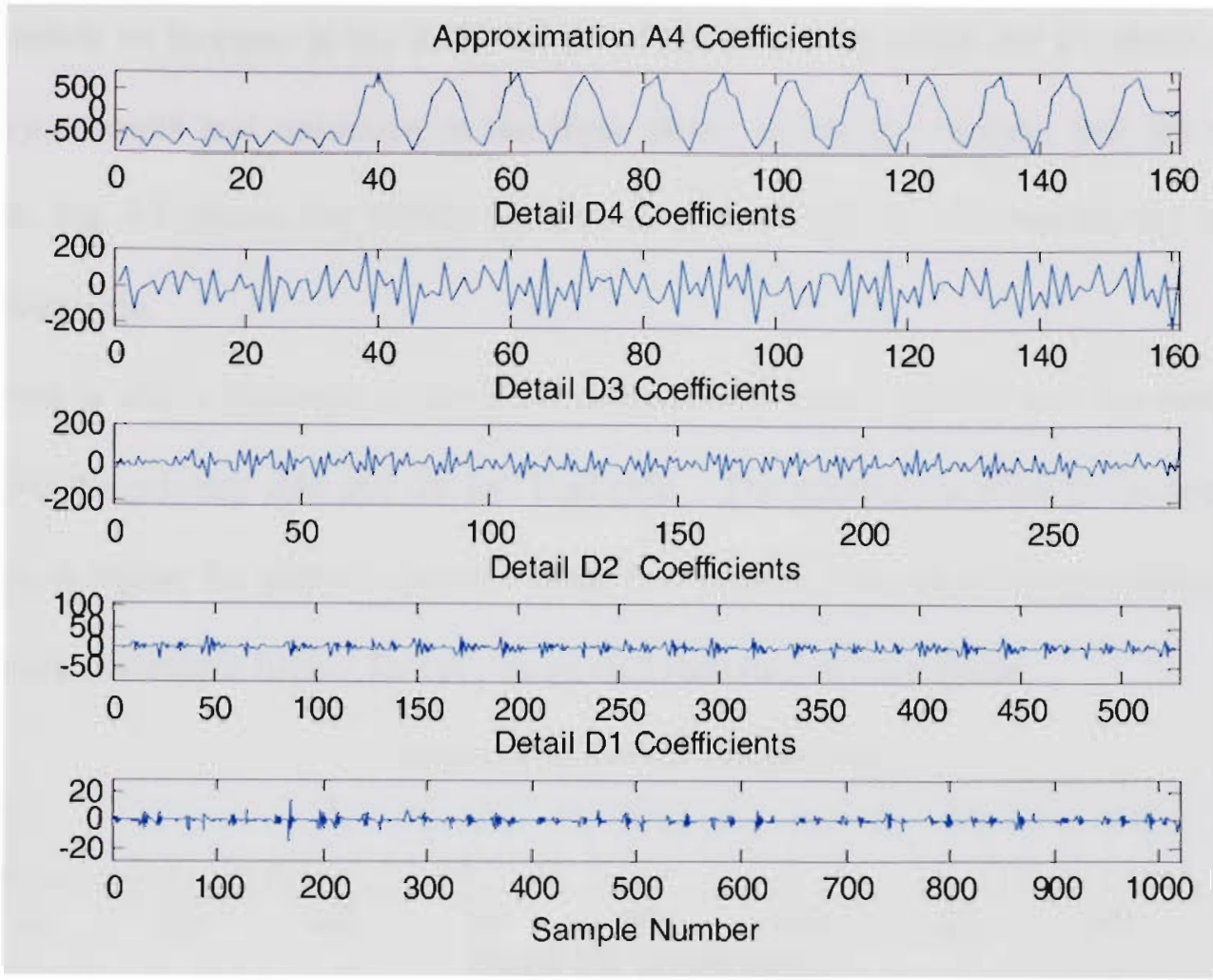

Fig.8.17. WTCs of phase a secondary current for arcing turn-to-turn fault on the primary of phase c

Fig 8.18 through Fig. 8.24 shows the WTCs of the secondary three phase currents, the dc side current, and the primary three phase currents for secondary turn-to-ground fault on phase c. Fig 8.25 through Fig. 8.30 shows the WTCs of the secondary three phase currents, the dc side current, and the primary three phase currents for secondary turn-toturn fault on phase $\mathrm{c}$. In this case of the turn-to-ground fault and turn-to-turn fault, on the secondary of the transformer the event appears to be localized in the D2, D3 .D4 and A4 detail coefficients. There is a reduction in the RMS value of the D2, D3, D4 and A4 on all of the secondary side currents. This declines increase with the number of faulty turns increase. 
There is an increase in the RMS values of the frequency bands A4 for phase a and c primary currents and reduction in the three phase secondary currents and the dc side current. Fig 4.5 shows the WTCs for turn-to-ground fault on the transformer phase c secondary side.

There is also a decrease in the RMS of frequency bands D2-D4, and increase in D1 RMS for the primary side and the DC load side. The relative decrease in the secondary currents is higher for phase $\mathrm{c}$ than the other two phases. The reduction percentage in the secondary currents is higher for turn-to-ground than turn-to-turn faults.

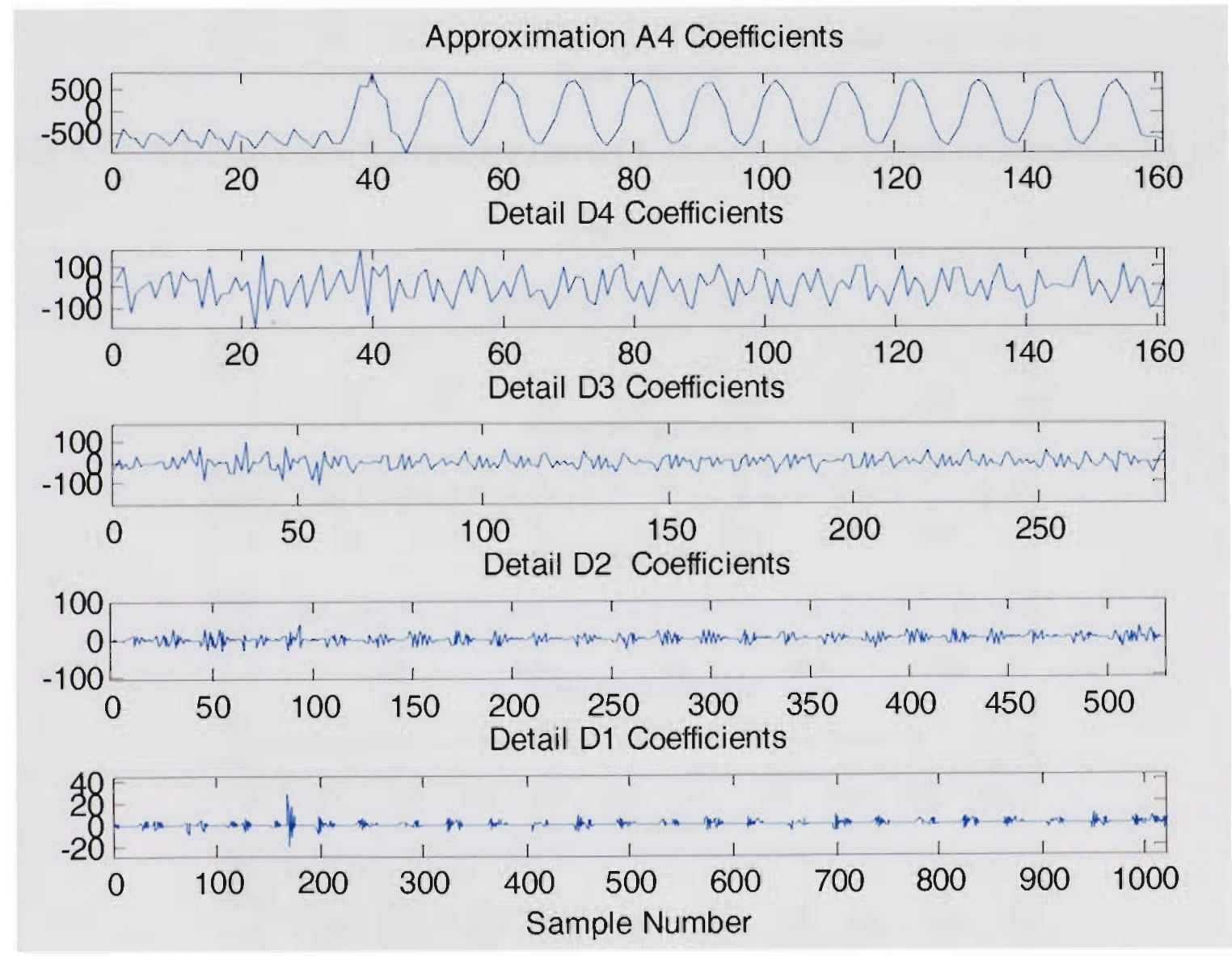

Fig.8.18. WTCs of phase a secondary current for turn-to-ground fault on the secondary of phase c 


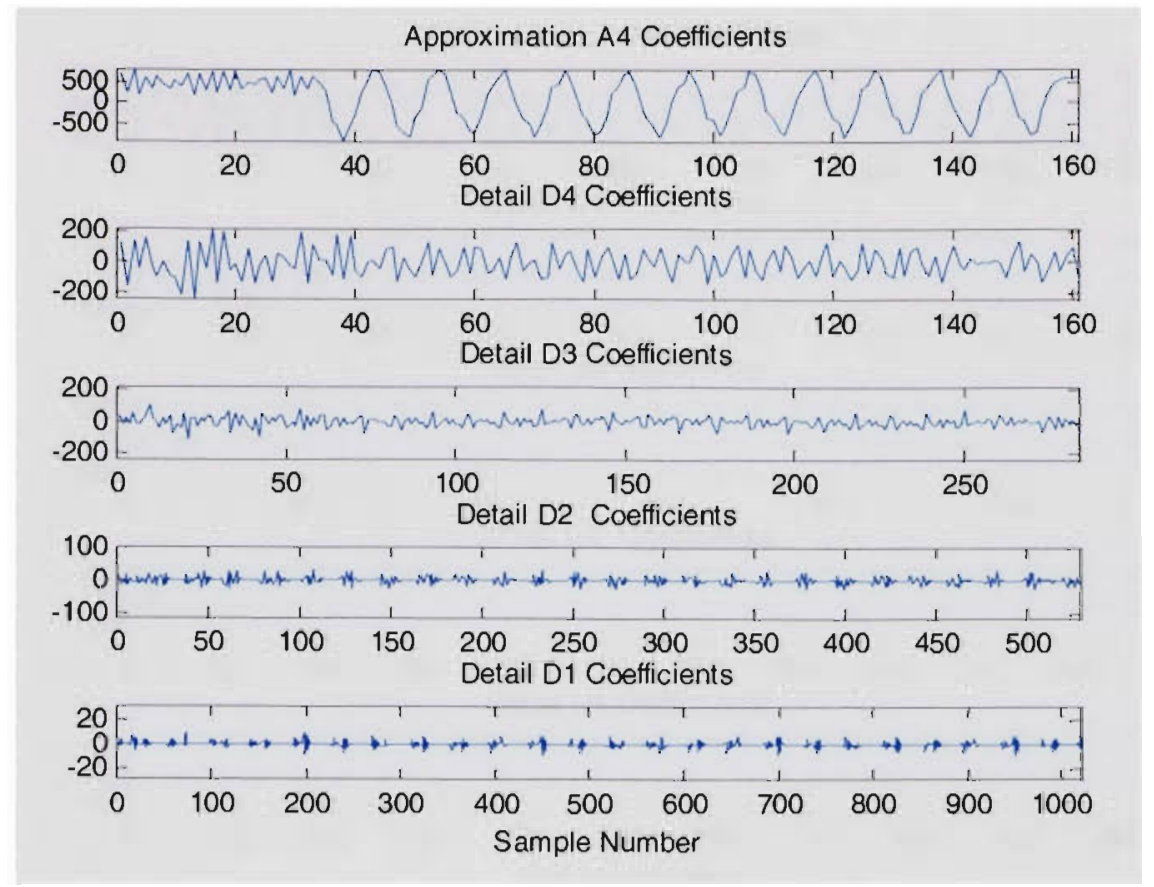

Fig.8.19. WTCs of phase b secondary current for turn-to-ground fault on the secondary of phase c

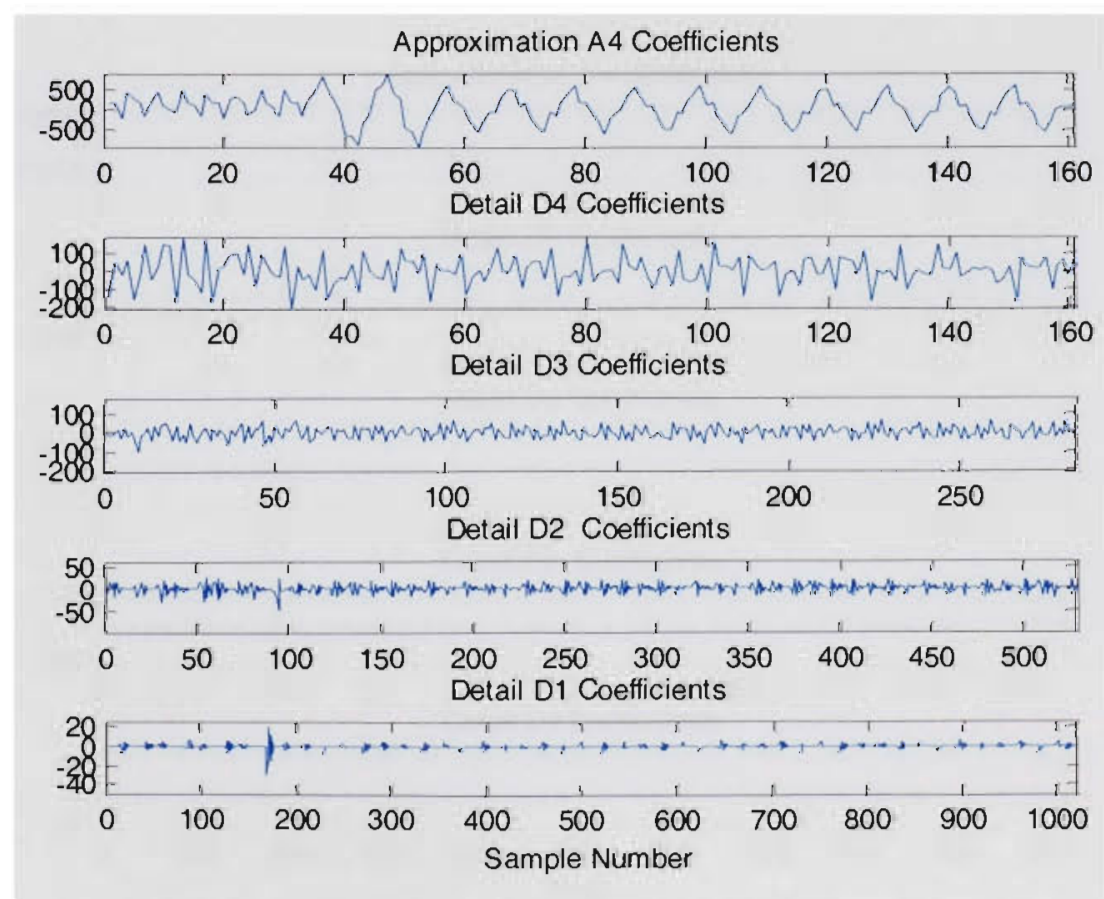

Fig.8.20. WTCs of phase c secondary current for turn-to-ground fault on the secondary of phase c 


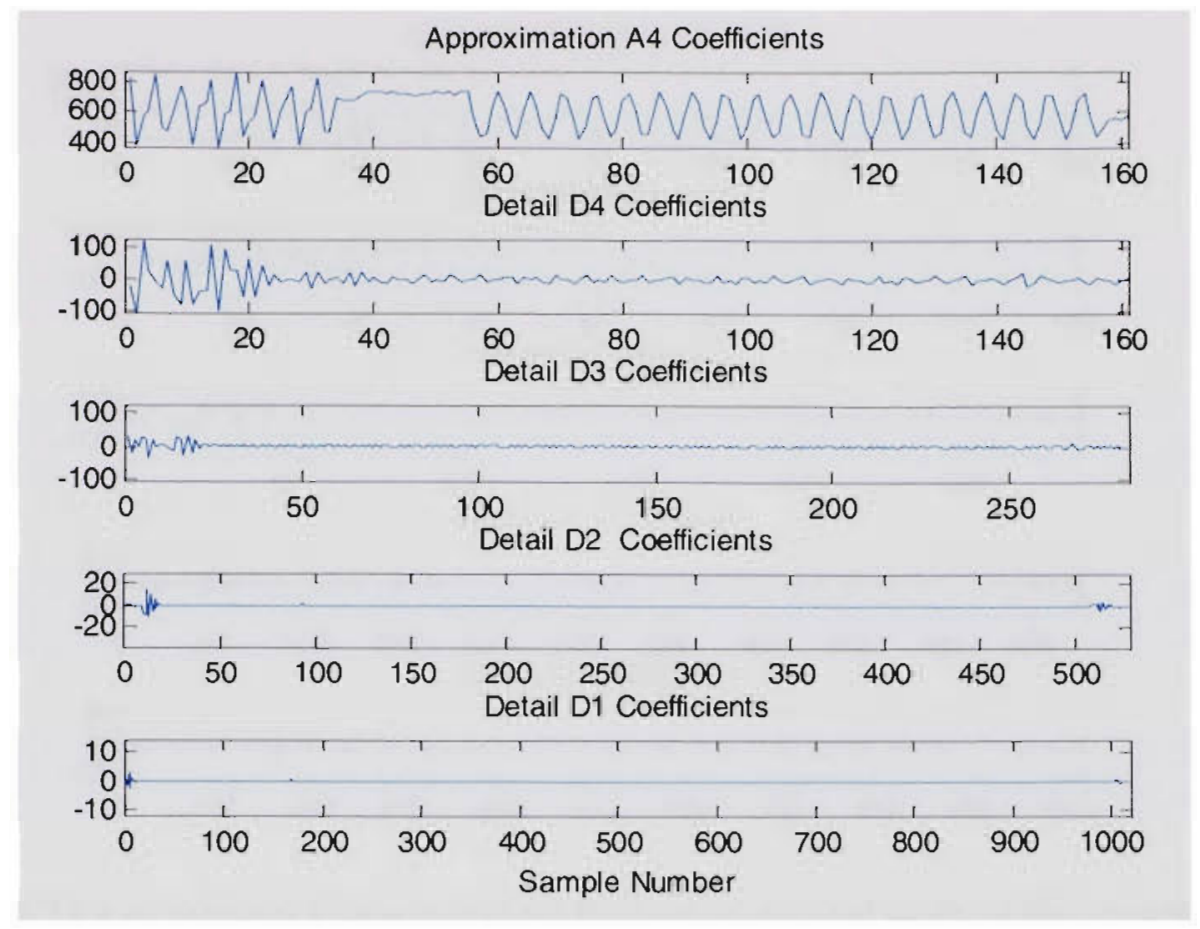

Fig.8.21. WTCs of the dc side current for turn-to-ground fault on the secondary of phase

C
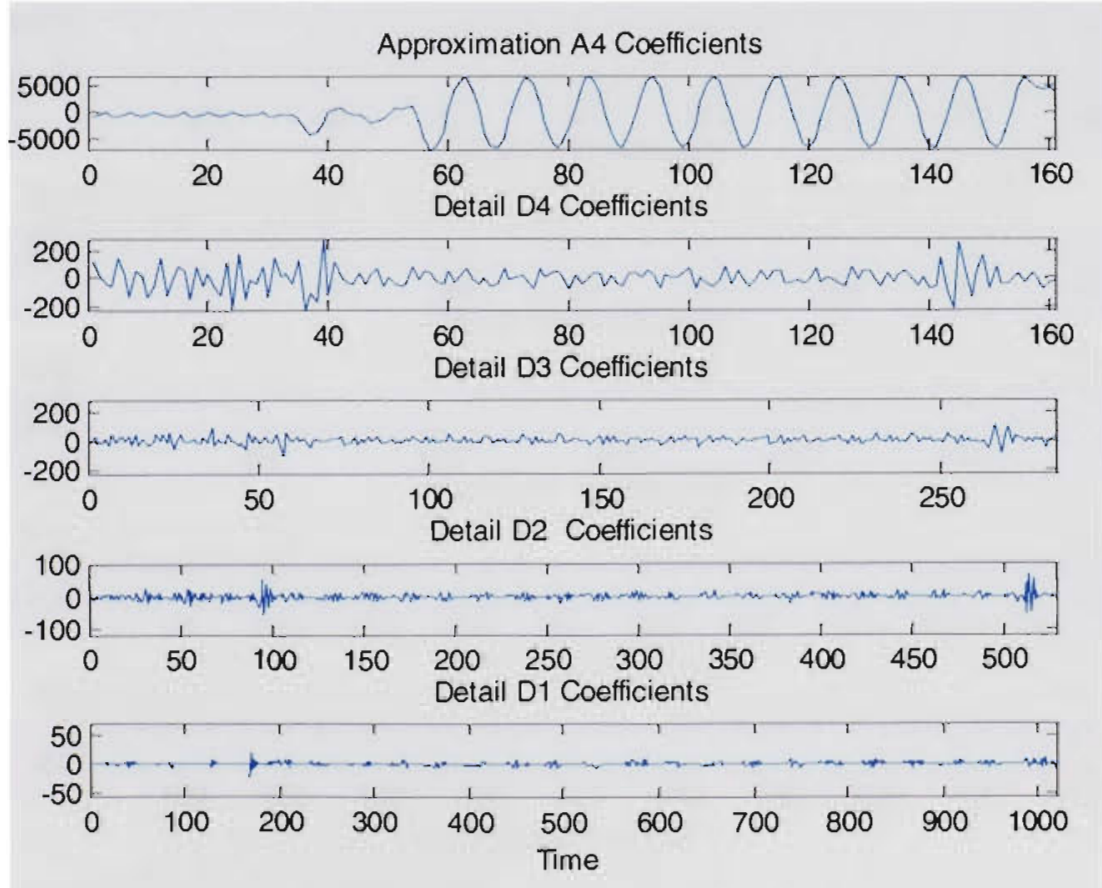

Fig.8.22. WTCs of primary Phase a current for turn-to-ground fault on the secondary of phase c 


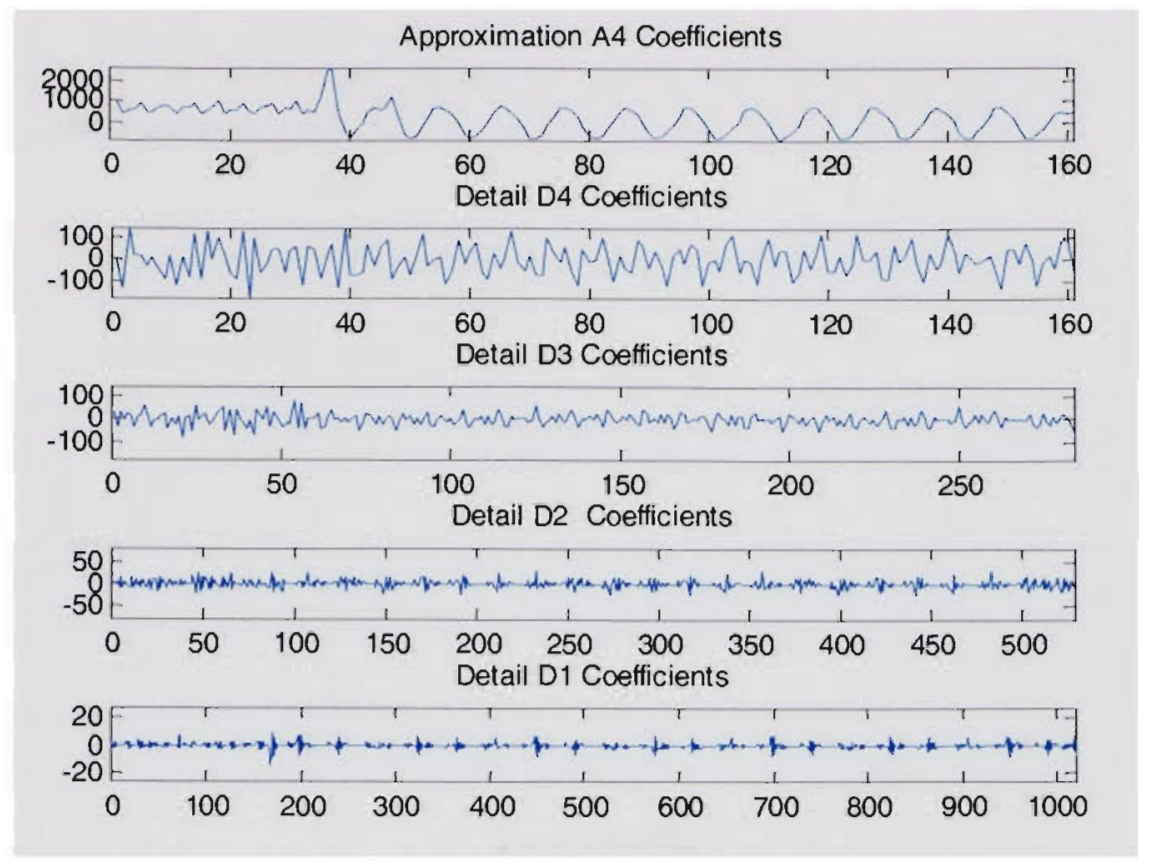

Fig.8.23. WTCs of primary Phase b current for turn-to-ground fault on the secondary of phase $\mathrm{c}$
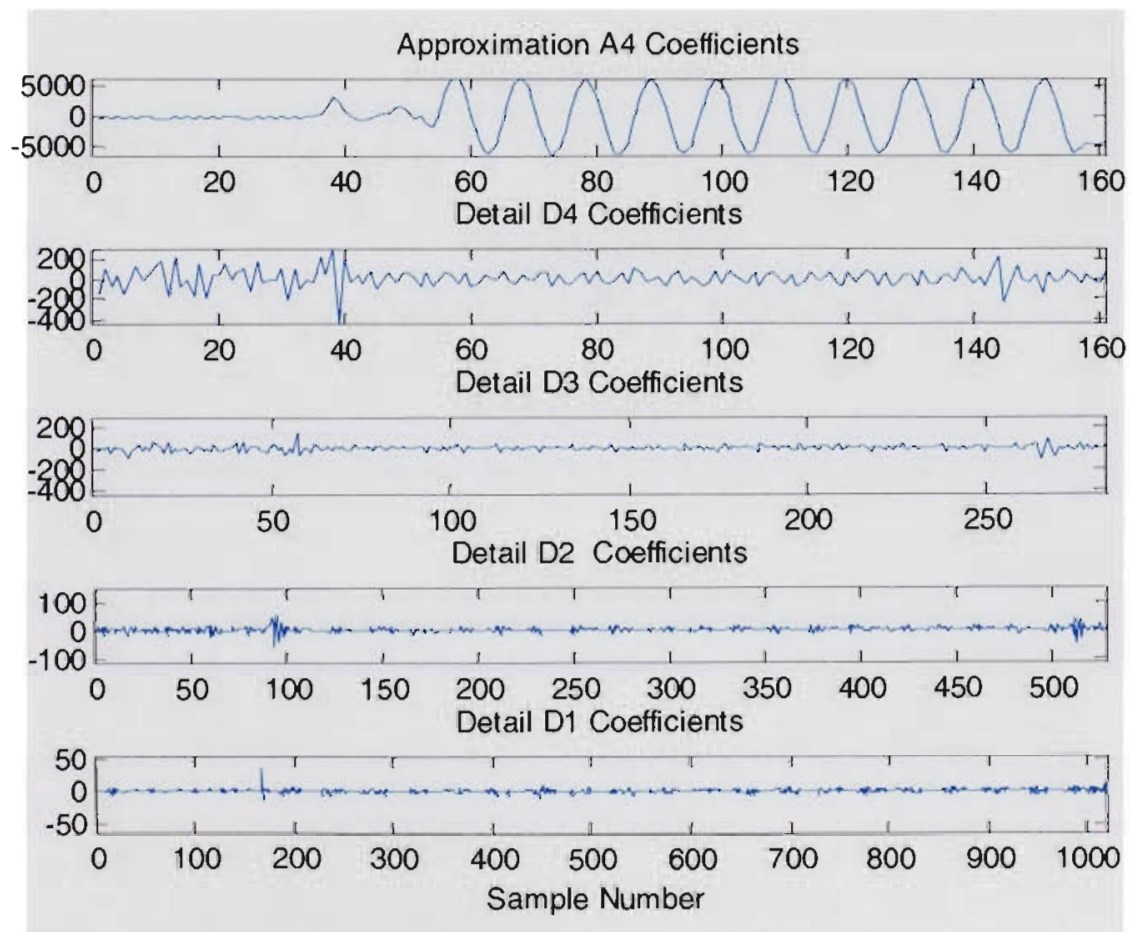

Fig. 8.24. WTCs of primary Phase C current for turn-to-ground fault on the secondary of phase c 


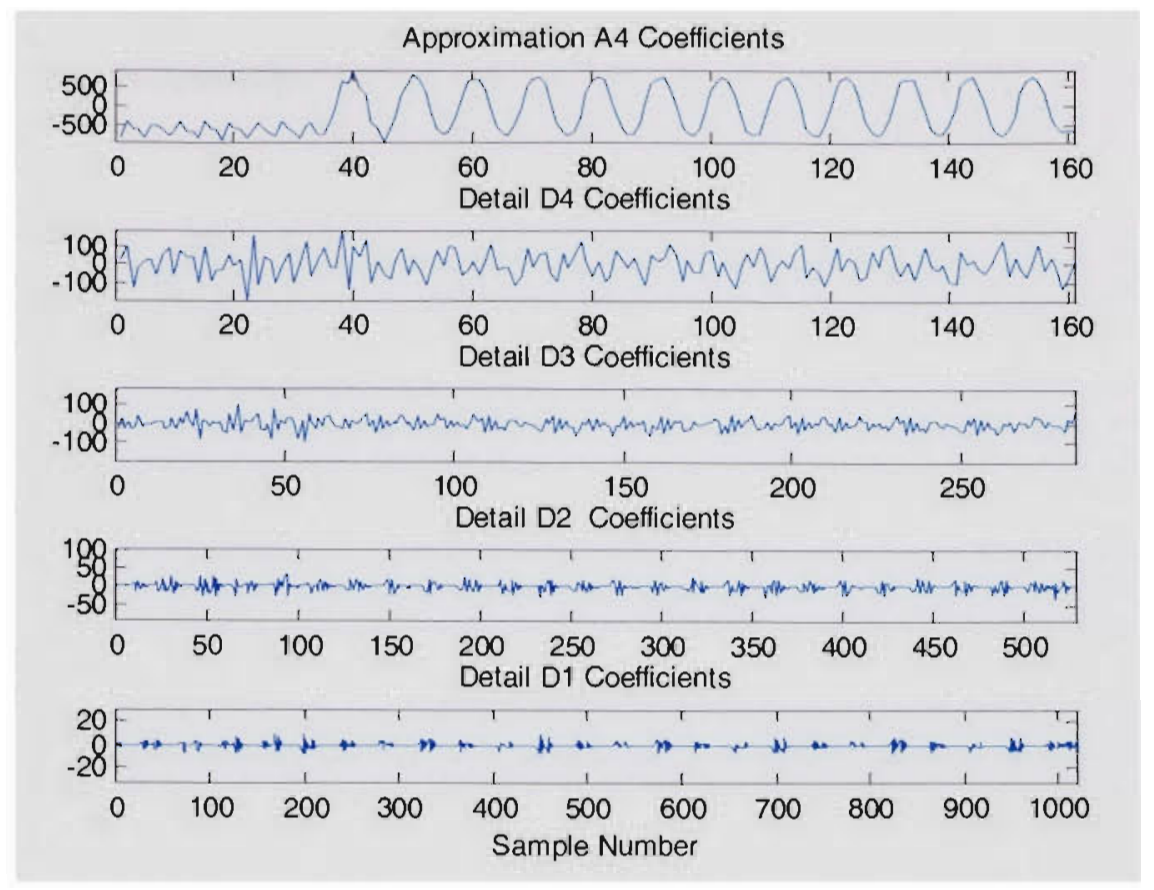

Fig.8.25. WTCs of phase a secondary current for turn-to-turn fault on the secondary of phase $\mathrm{c}$

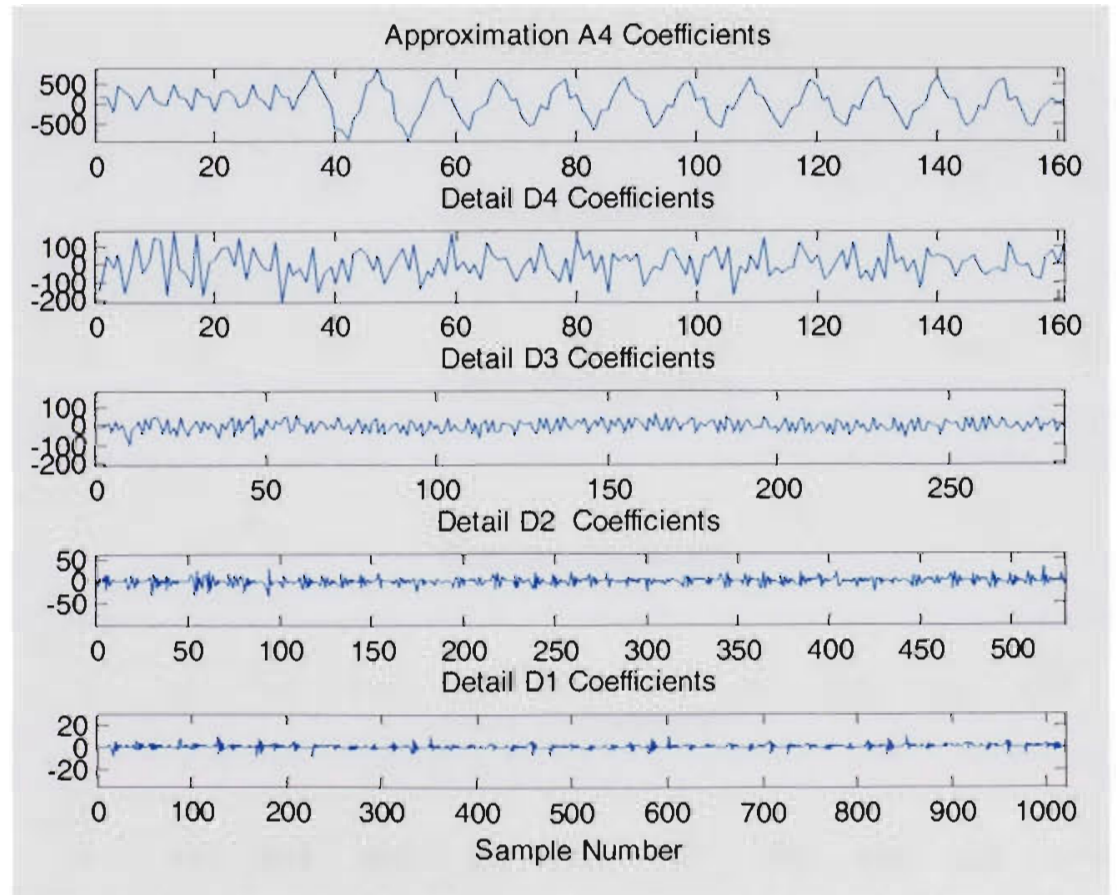

Fig.8.26. WTCs of phase $\mathrm{c}$ secondary current for turn-to-turn fault on the secondary of phase $\mathrm{c}$ 


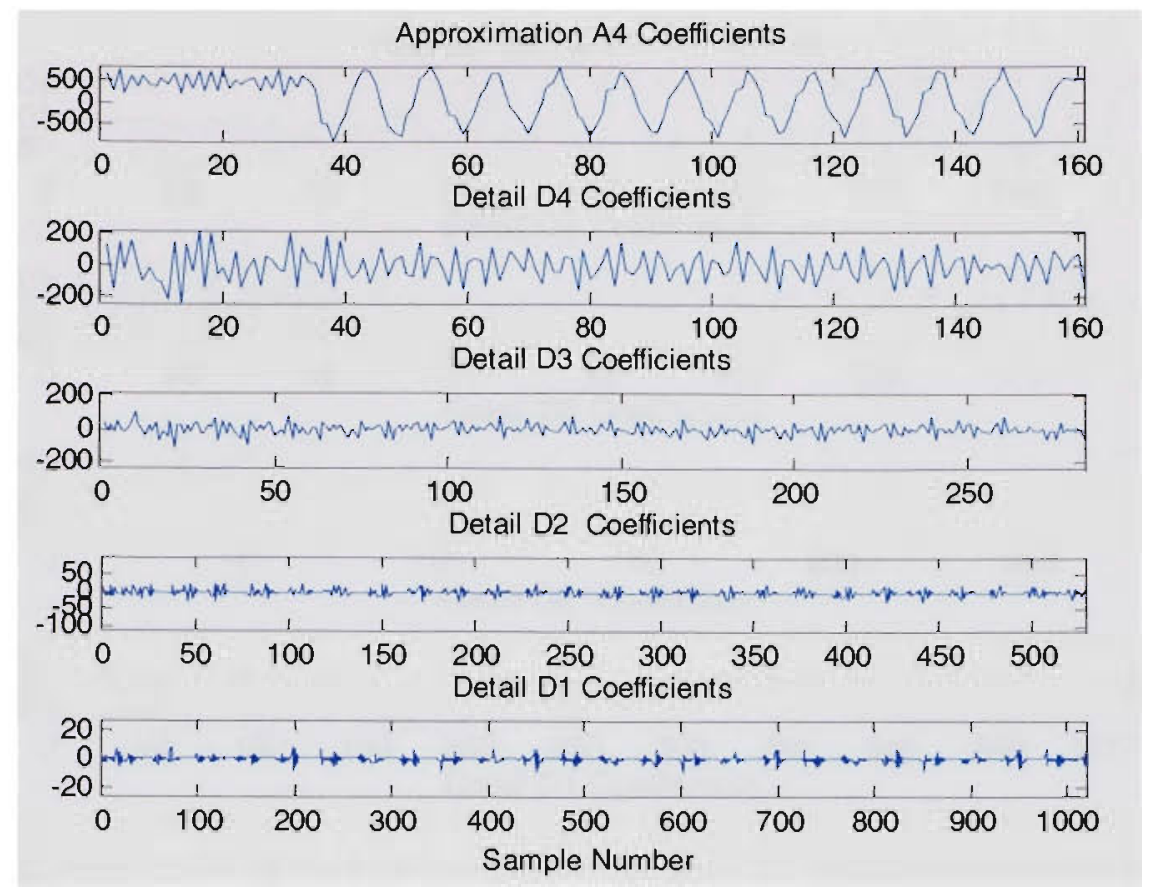

Fig.8.27. WTCs of phase b secondary current for turn-to-ground fault on the secondary of phase c

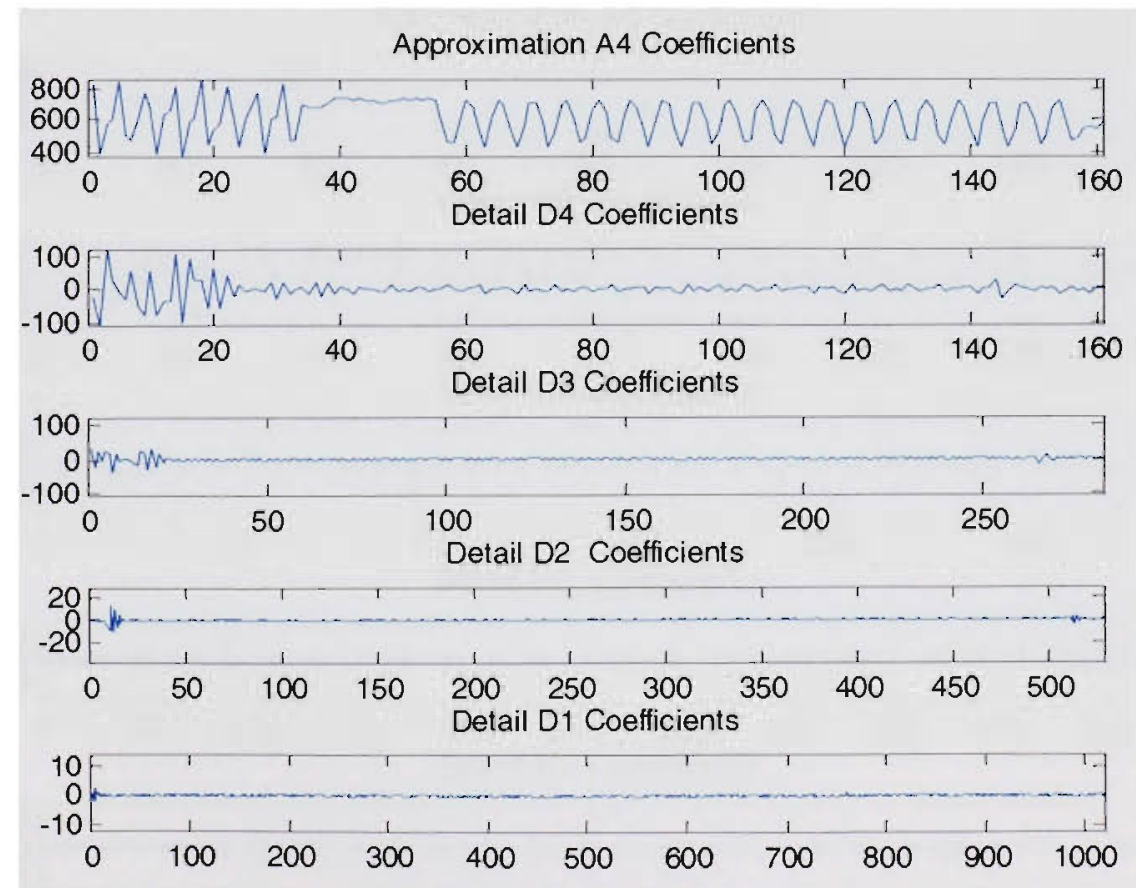

Fig.8.28. WTCs of the dc side current for turn-to-turn fault on the secondary of phase c 


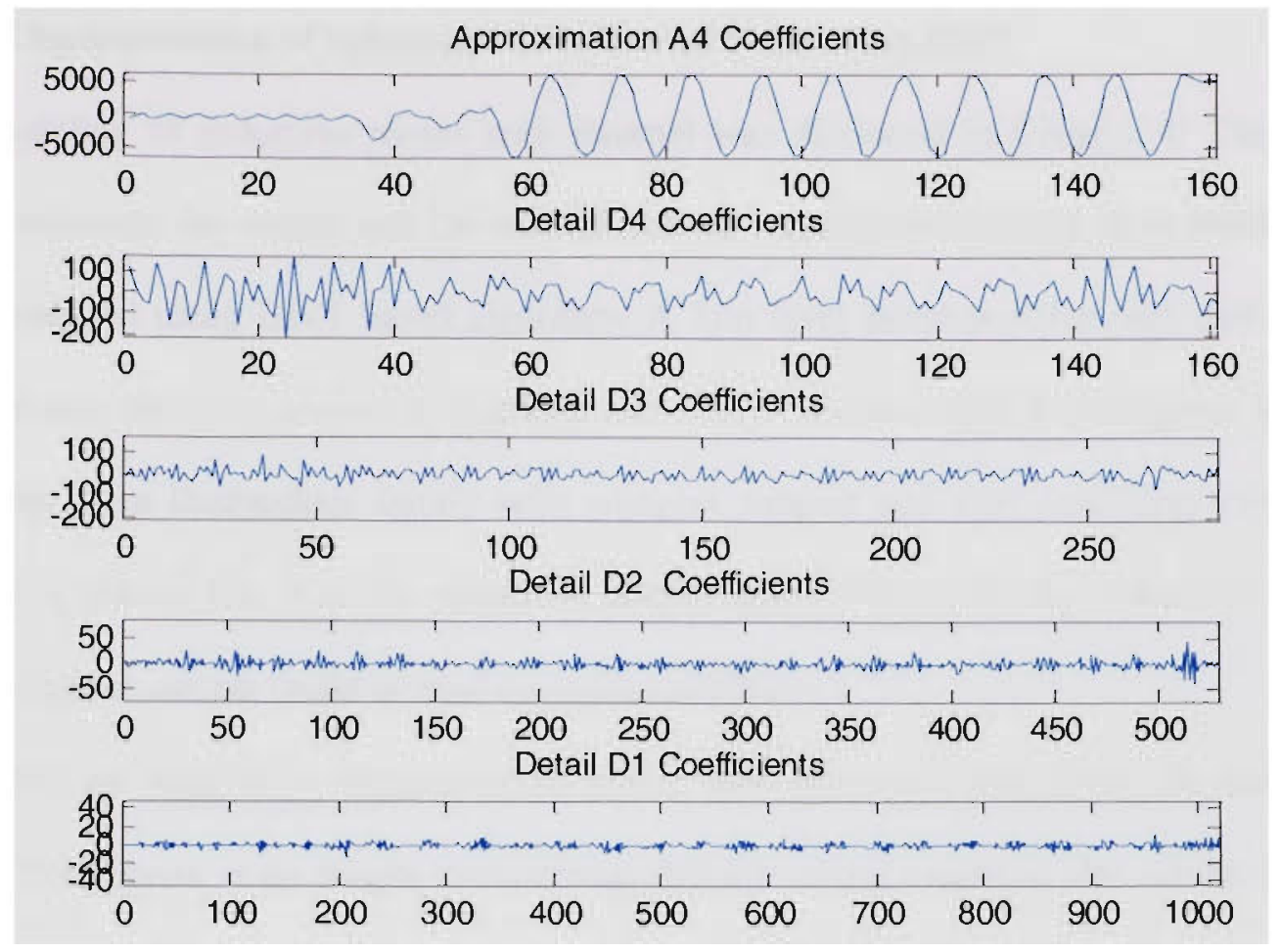

Fig.8.29. WTCs of primary Phase a current for phase c secondary turn-to-turn fault

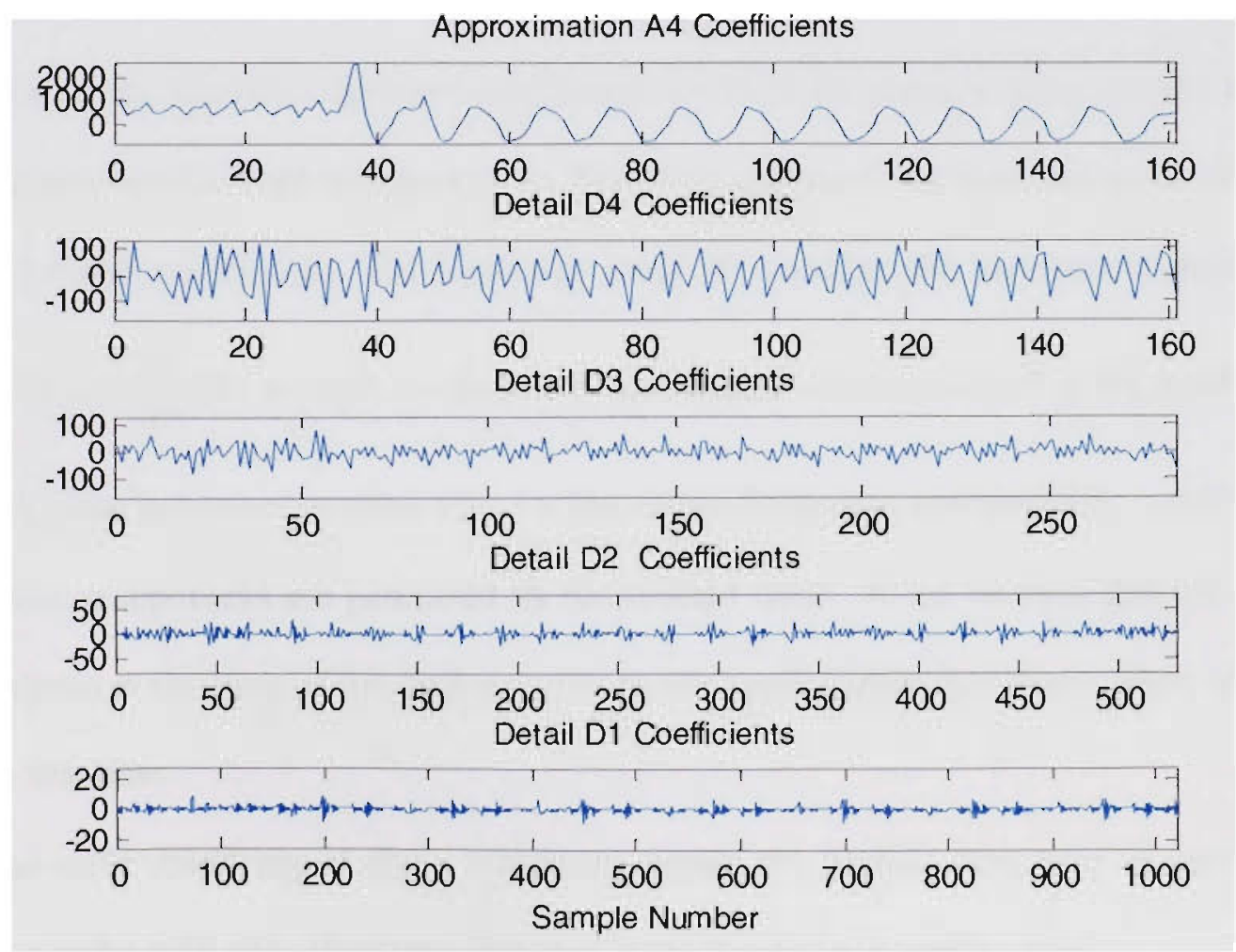

Fig.8.30. WTCs of primary phase b current for phase $\mathrm{c}$ secondary turn-to-turn fault 


\subsubsection{Characterization of induction motor internal faults using DWT}

Modeling of induction motor with internal was discussed in Chapter 4 . The three phase currents, the torque and the neutral current waveforms obtained from FEM were then analyzed using DWT based algorithm. A four level decomposition with db4 as the mother wavelet was selected to perform our study. Wavelet $\mathrm{db} 4$ is orthogonal mother wavelets from Daubechies family with compact support and four vanishing moments. Fig. 8.31 shows the Wavelet transform coefficients (WTCs) for the induction motor phase a stator current under normal operating condition.

There are significant changes in the motor three phase currents due to shorted turns fault. The current is no longer symmetrical and the strong magnetic flux of the faulted coil will influence the currents in other phases. Besides the current increase in phase a there will be a relative increase in phase $\mathrm{c}$ and a relative decrease in phase $\mathrm{b}$ current. Fig. 8.32 shows the Wavelet transform coefficients (WTCs) for phase a stator current for 44 shorted turns under ideal voltage source. The event appears to be localized in the D1, D4 and A4 detail coefficients. The frequency band D1 contains the harmonic components given by $\left(m\left(\frac{2 N}{P}\right)(1-s) \pm 1\right) f$, where $\mathrm{N}$ is the number of rotor bars, $\mathrm{P}$ is the number of poles, $\mathrm{s}$ is the induction machine slip, $f$ is the source frequency, and $\mathrm{m}=1,2,3, \ldots$ [90-94].

These components are generated by the shorted turns. It can be seen that the DWT coefficients at the time of the fault occurrence are much higher than their values after or before this time.

The scale detail signal (D1), which represents the highest frequency components, shows a spike with very short time duration at the disturbance starting time. 


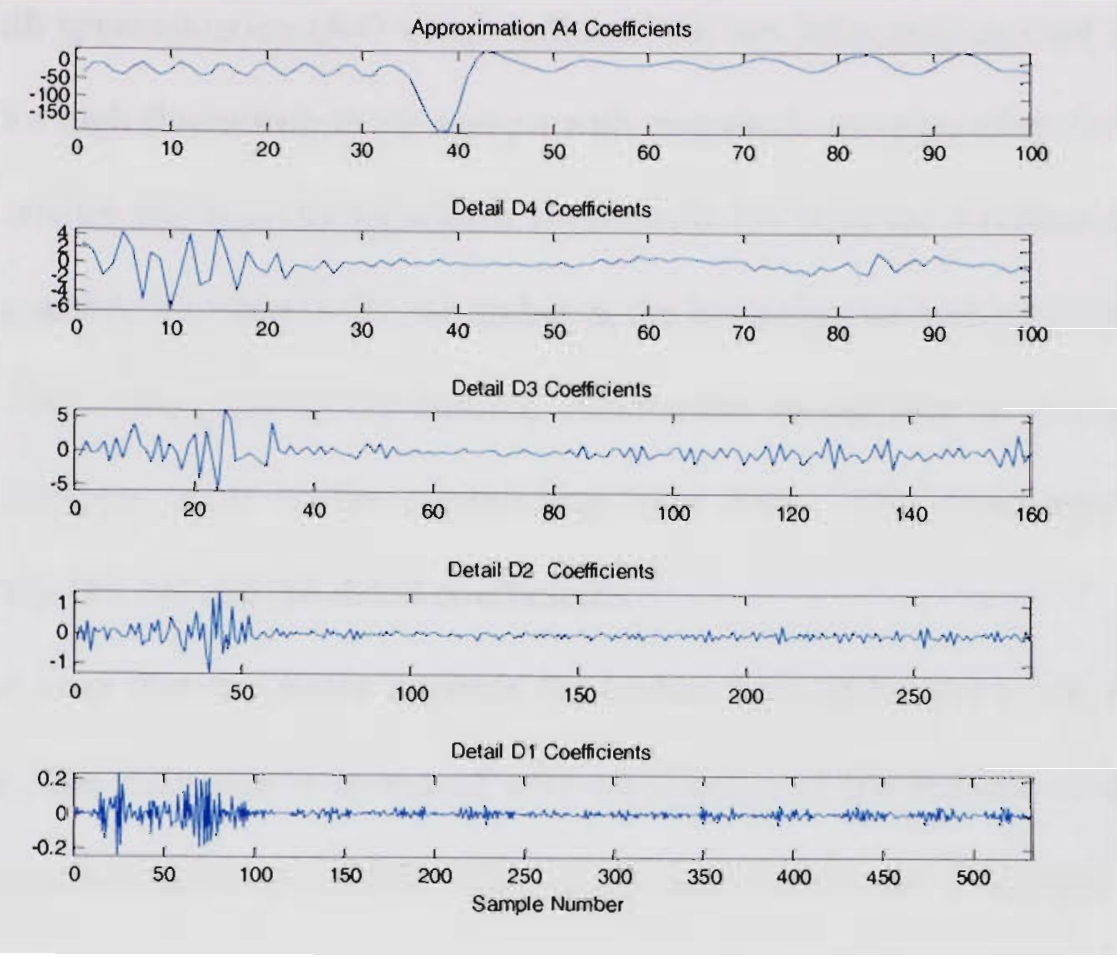

Fig.8.31. WTCs of phase a current for healthy motor under ideal voltage supply

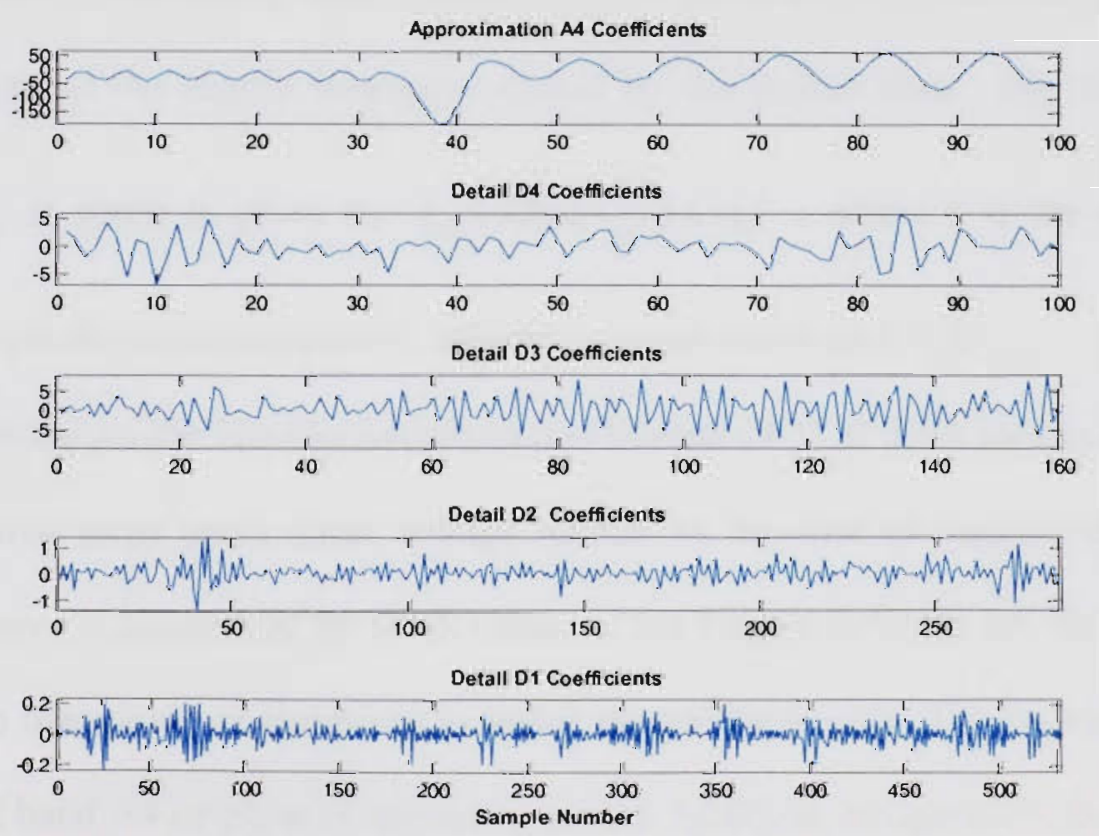

Fig. 8.32. WTCs of the induction motor phase a current for 44 shorted stator turns under ideal voltage supply 
The fourth approximation (A4) which indicates the low frequency content of the fault signal shows a high fluctuation in the current with magnitude increase after the fault.

Figure 8.33 shows the Wavelet transform coefficients (WTCs) for 4 broken bars under ideal voltage source. In figure 8.33 , the spikes at the beginning of each of the scale detail signals (D1-D4) correspond to the starting disturbance. In the case of broken bar, the current redistributes itself in the surrounding rotor bars. The event appears to be localized in the D2, D4 and A4 detail coefficients.

It can be seen that the stator currents for broken bars faults are lower than in the healthy case. The reduction is increased with an increase in the number of faulty bars. The fourth approximation (A4) RMS value which indicates the low frequency content of the fault signal shows a magnitude decrease after the fault. This decrease ranges between $3-25 \%$ compared to the healthy case. The reduction is due to the left sidebands frequency components around the supply frequency caused by the broken bars. The side bands frequency $f_{b b}$ is given is given by $f_{b b}=\left(2 m\left(\frac{1-s}{P}\right) \pm s\right) f$, where $\mathrm{s}$ is the induction machine slip, $f$ is the source frequency, and the harmonic index $\mathrm{m}$ is $1,2,3, \ldots$

Figure 8.34 shows the Wavelet transform coefficients (WTCs) for 4 broken bars with 44 stator shorted turns under ideal voltage source. In the case of shorted turns with broken bars, there is increase in the RMS values of the frequency bands A4, D4, D3, and D1 but less in magnitude as compared to stator shorted turns only. The decrease in the RMS value of band A4 of phase A current is around 3-20\% as compared to short circuit only case. 


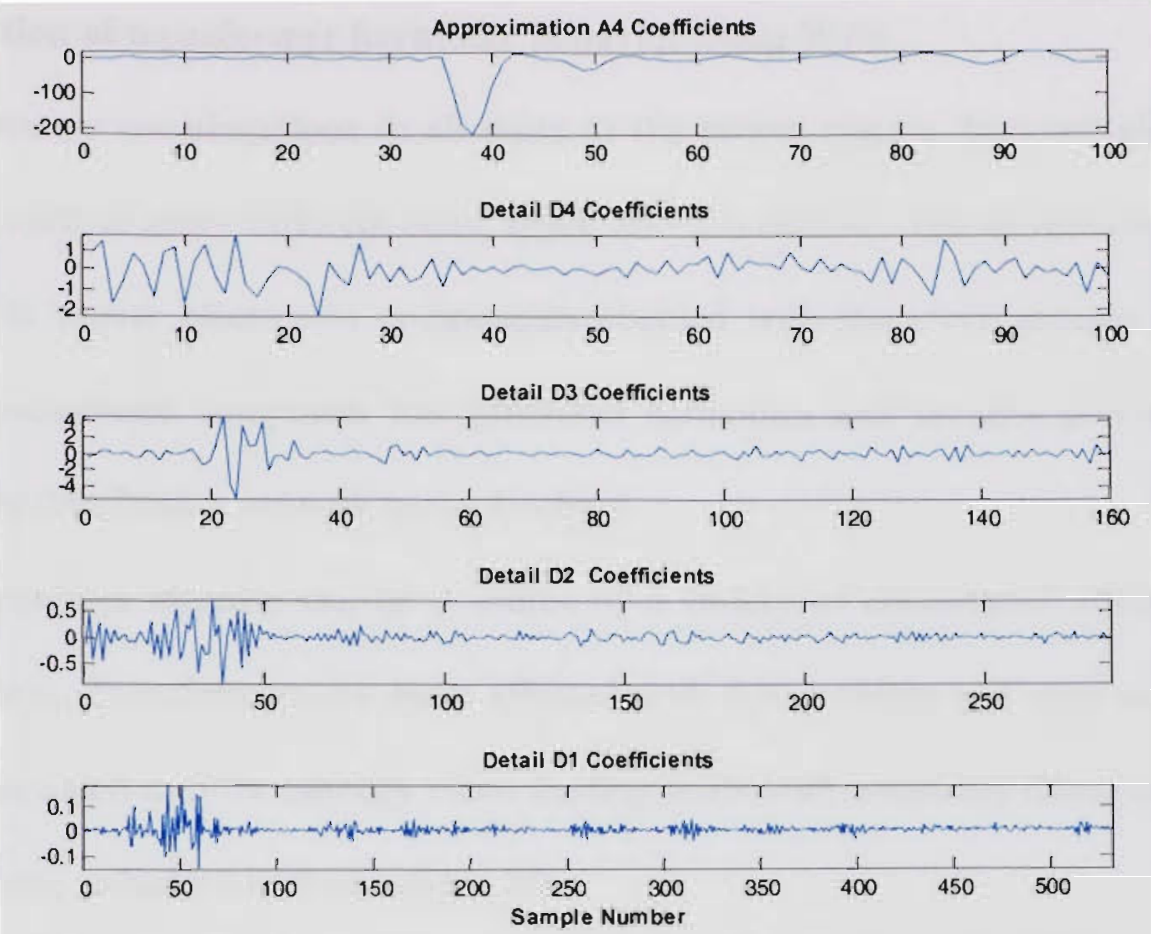

Fig. 8.33. WTCs of phase a current for 4 broken bars under ideal voltage supply

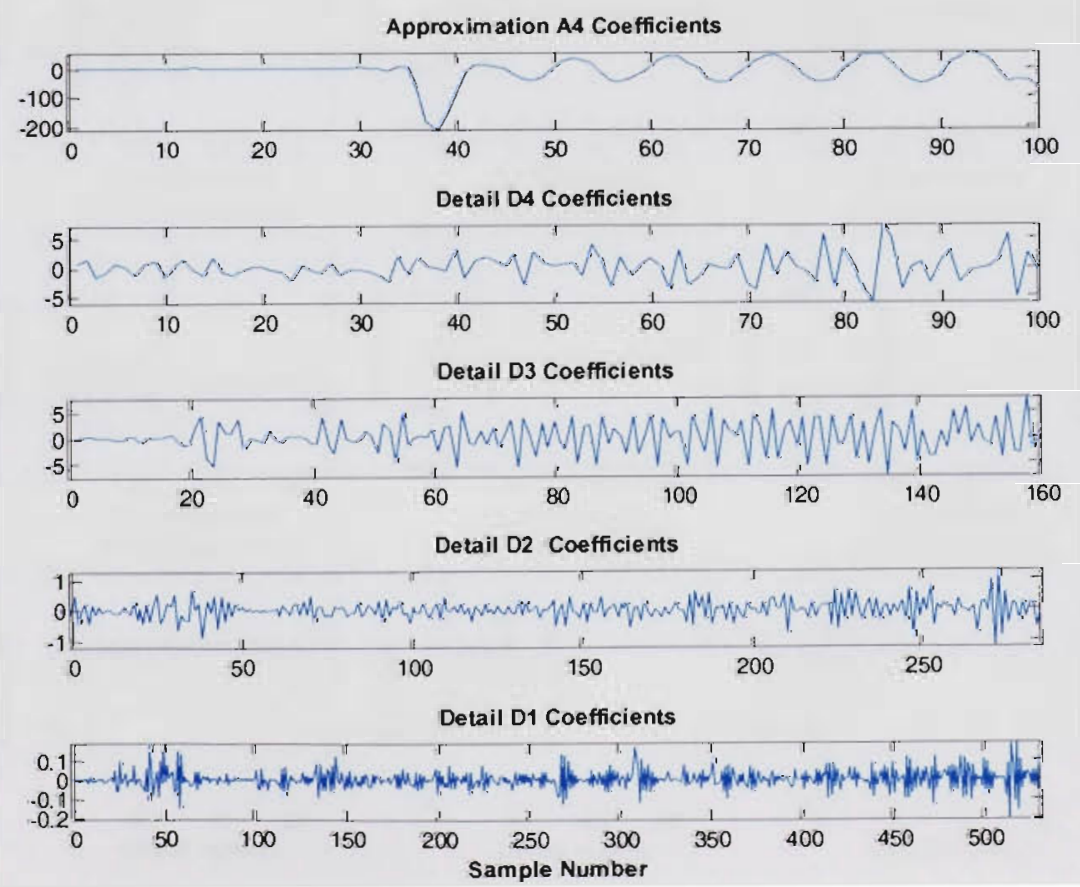

Fig.8.34. WTCs of phase a current for 44 stator shorted turns with 4 broken bars under ideal voltage supply 


\subsection{Evaluation of transformer harmonic behavior using WPT}

Transformers are ubiquitous in all parts of the power system, between all voltage levels, and exist in many different sizes, types, and connections. The increase utilization of non-linear power electronics components coupled with the more prevalent use of microprocessor-based equipment has generated harmonics and brought power quality issues on the distribution network to the forefront.

The harmonics increase can be a source of a variety of undesirable effects in the energy system. Transformers are most affected with this problem and may need to be derated to as much as $50 \%$ capacity when feeding loads with extremely distorted current waveforms and reduction in their service life.
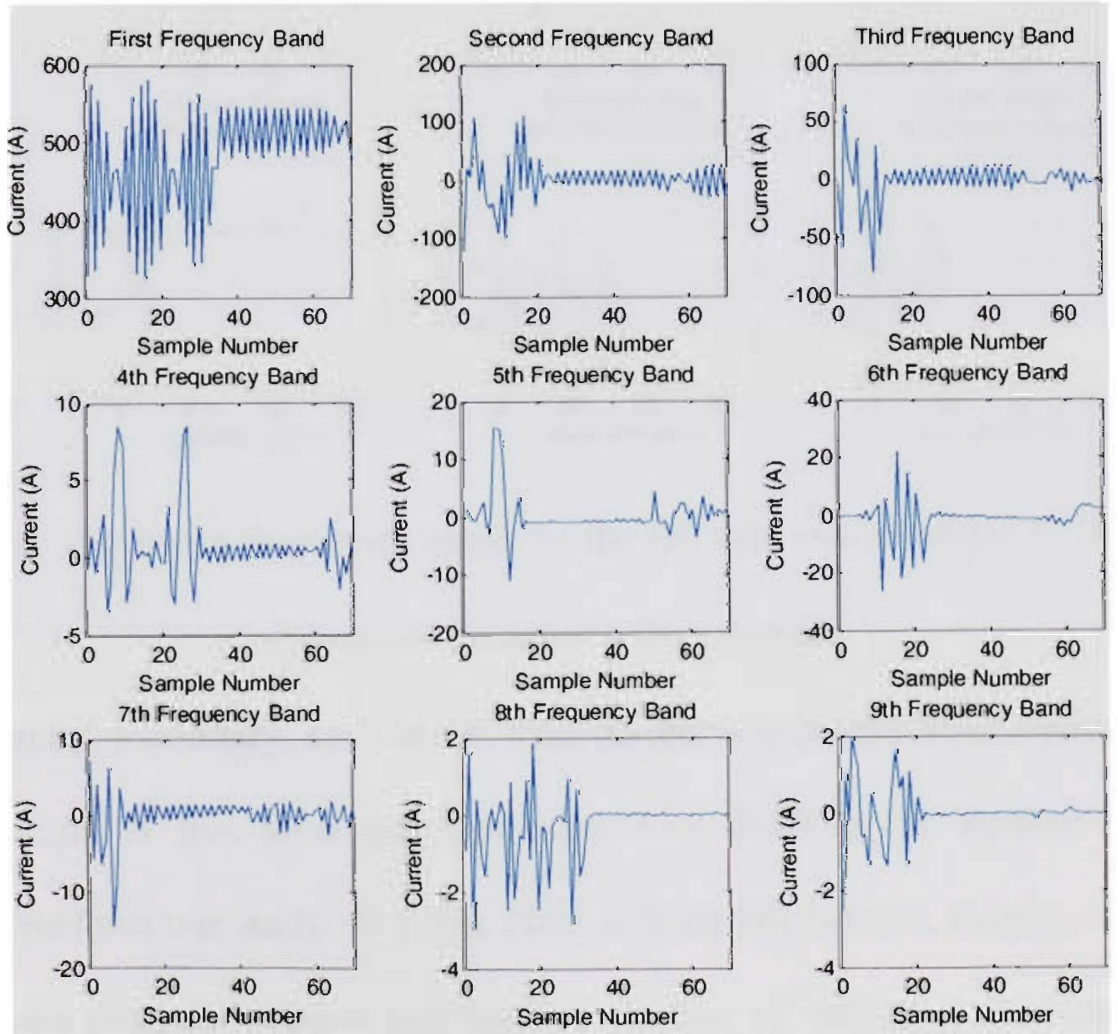

Fig. 8.35. The first 6 frequency bands for the DC side current under $3^{\text {rd }}$ peaking harmonic distorted voltage supply. 
The IEEE/ANSI C57.110-1998 standards states that a transformer subject to nonsinusoidal load current having more than $5 \%$ total harmonics distortion needs to be derated [52]. The harmonic behavior of the transformer was evaluated by using FEM as descried in chapter 3 .
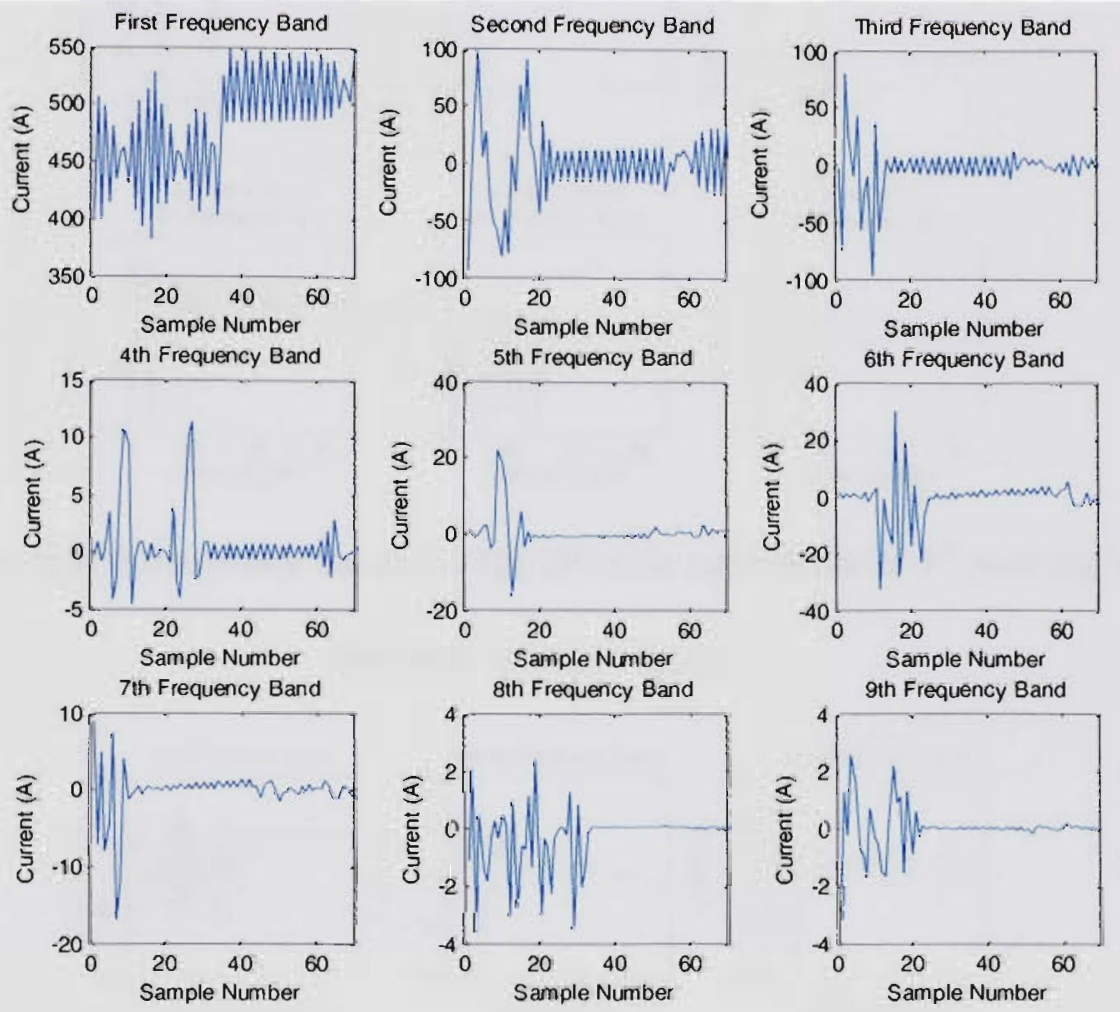

Fig.8.36. The first 6 frequency bands for the DC side current under $3^{\text {rd }}$ flattened harmonic distorted voltage supply.

The primary, secondary, and the DC side current waveforms were analyzed by using WPT algorithm. A five level decomposition with $\mathrm{db} 20$ as the mother wavelet was selected to perform our study. Wavelet db20 is from Daubechies Family of Orthogonal Wavelets with compact support and highest number of vanishing moments. This will decompose the signal into 32 frequency band. 

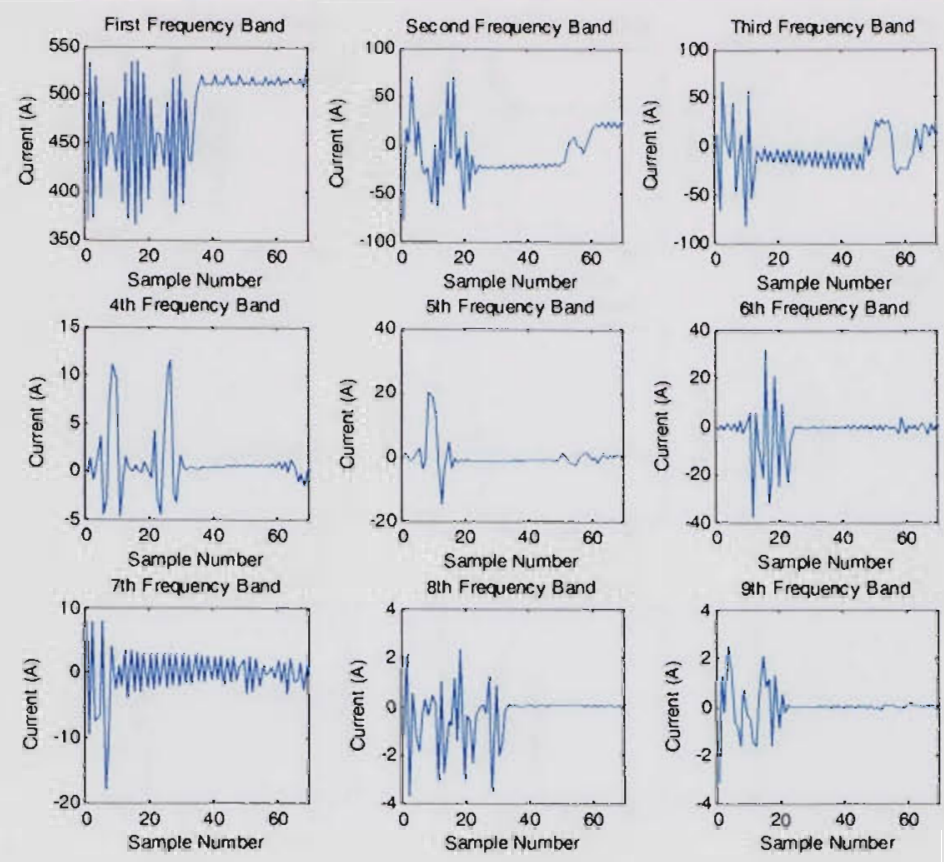

Fig. 8.37. The first 6 frequency bands for the DC side current under $5^{\text {th }}$ peaking harmonic distorted voltage supply.

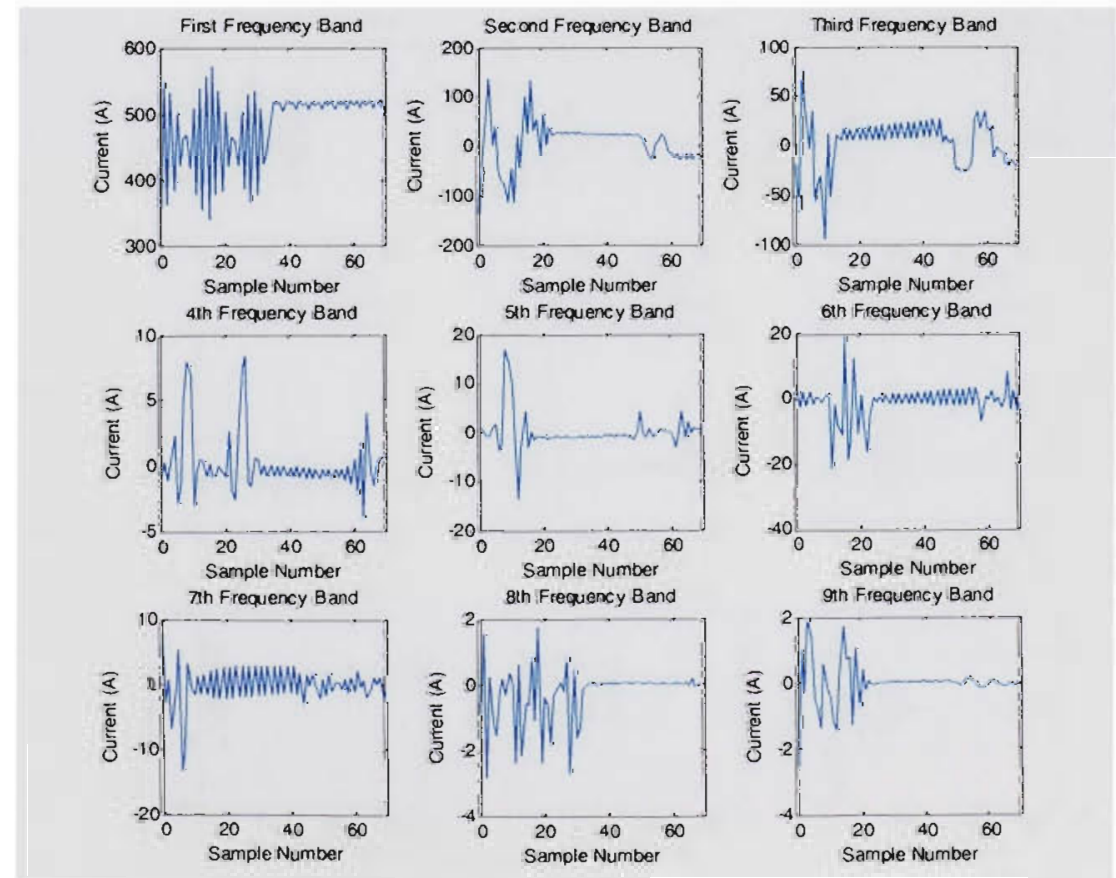

Fig. 8.38. The first 6 frequency bands for the DC side current under $5^{\text {th }}$ flattened harmonic distorted voltage supply. 

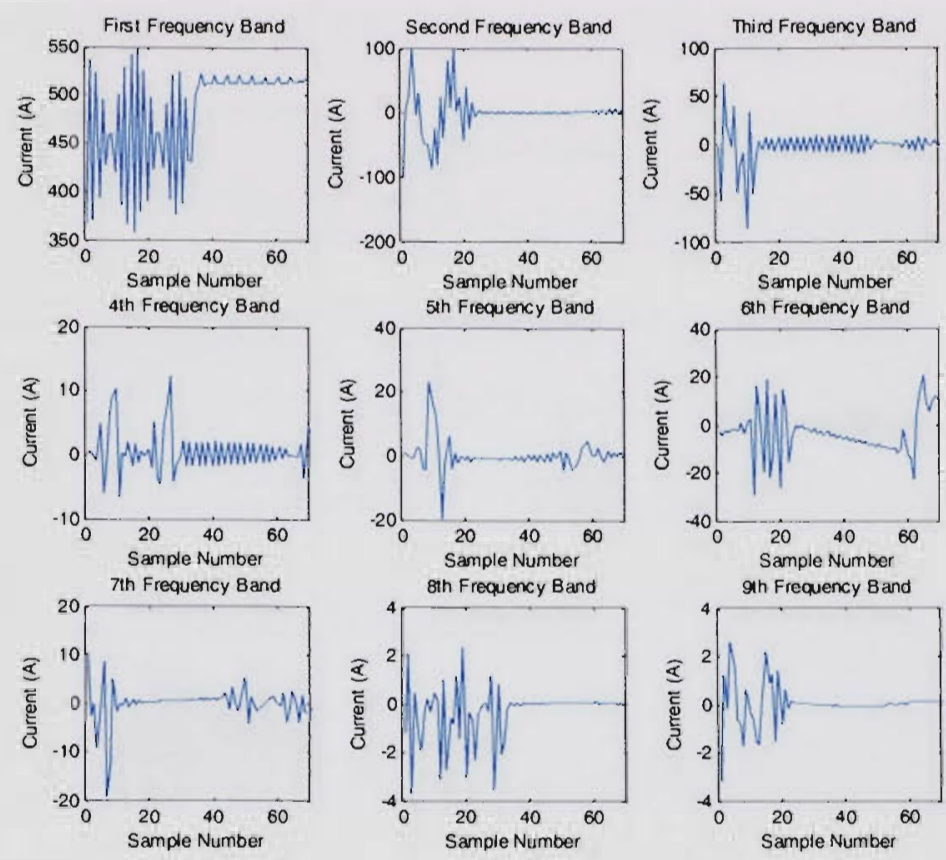

Fig. 8.39. The first 6 frequency bands for the DC side current under $9^{\text {th }}$ peaking harmonic distorted voltage supply.
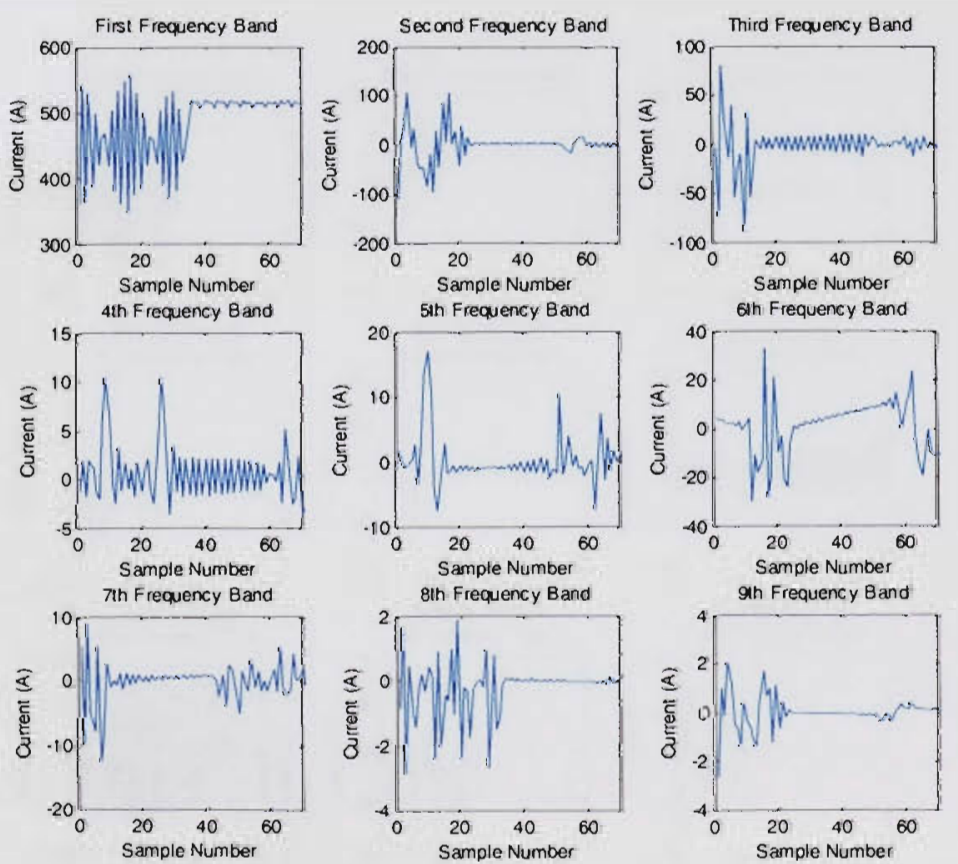

Fig. 8.40. The first 6 frequency bands for the DC side current under $9^{\text {th }}$ flattened harmonic distorted voltage supply. 

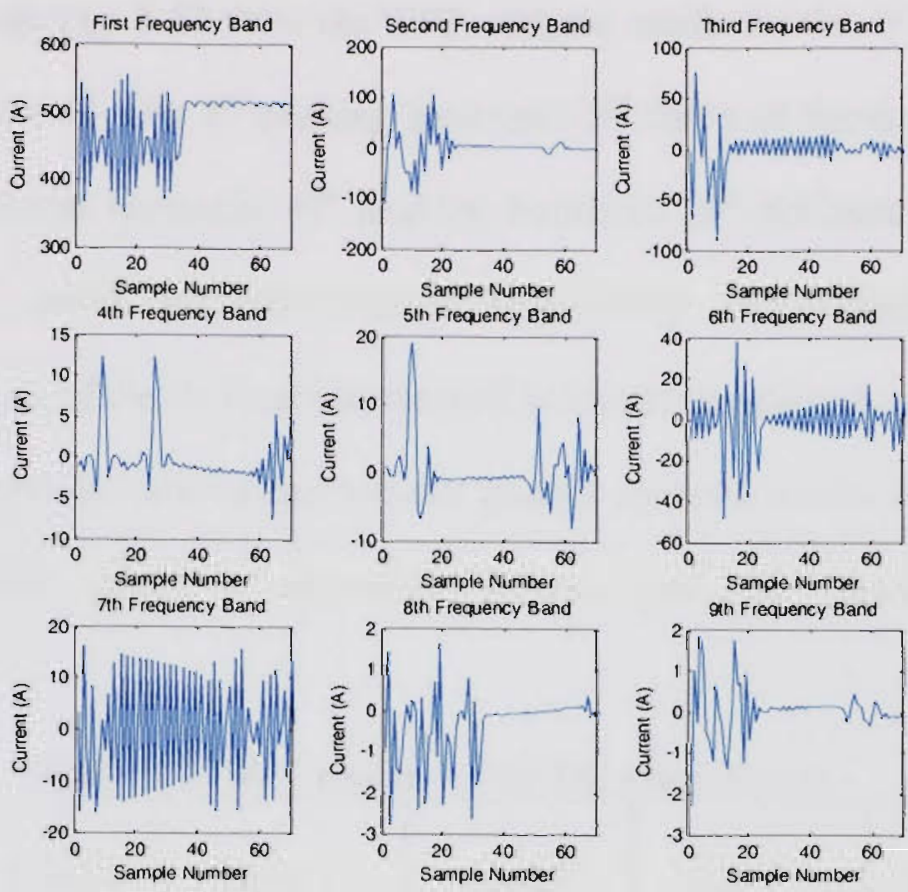

Fig.8.41. The first 6 frequency bands for the DC side current under $11^{\text {th }}$ peaking harmonic distorted voltage supply.
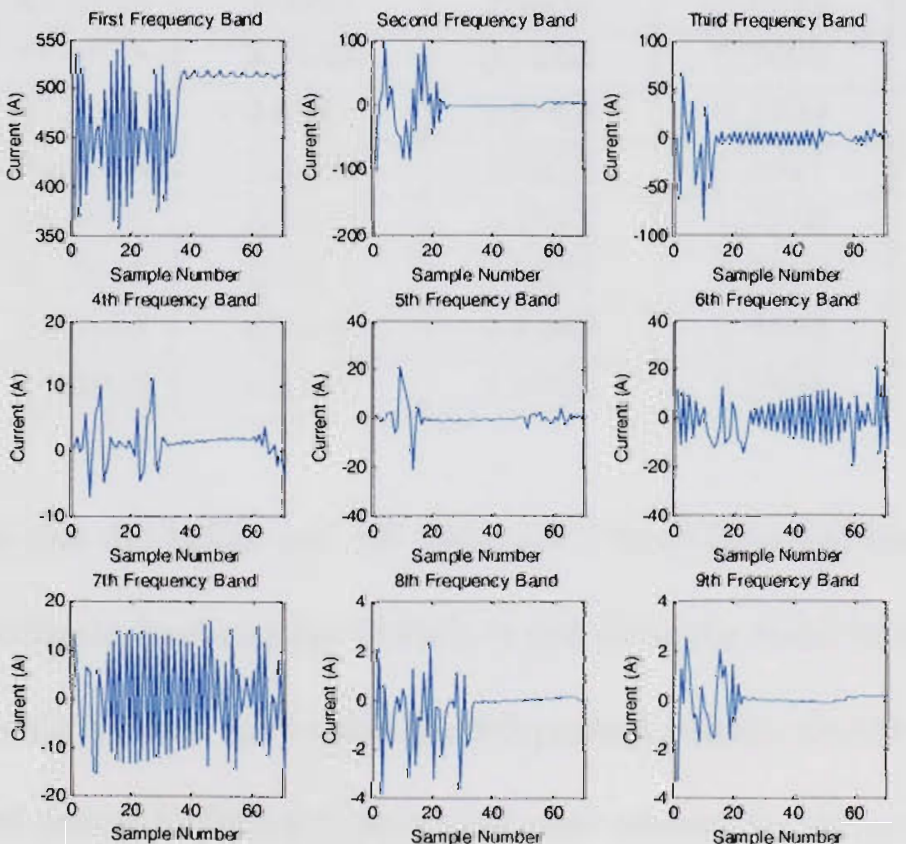

Fig.8.42. The first 6 frequency bands for the DC side current under $11^{\text {th }}$ flattened harmonic distorted voltage supply. 
Fig. 8.35 through Fig. 8.42 show the WPT analysis results for the dc load current for voltage source distorted with $3^{\text {rd }}$ peaking harmonic, $3^{\text {rd }}$ flattened harmonic, $5^{\text {th }}$ peaking harmonic, $5^{\text {th }}$ flattened harmonic, $9^{\text {th }}$ peaking harmonic, $9^{\text {th }}$ flattened harmonic, $11^{\text {th }}$ peaking harmonic, and $11^{\text {th }}$ flattened harmonic respectively. In this figure, the spikes at the beginning of each of the six bands correspond to the starting disturbance.

Tables 8-1, 8-2, and 8-3 shows the wavelet packet analysis results for the DC side currents, transformer phase a secondary current, and the magnetizing current respectively.

Table 8-1 WPT results for the DC side currents

\begin{tabular}{|c|c|c|c|c|c|}
\hline & $\begin{array}{c}\text { Band 0 } \\
0-120 \mathrm{~Hz}\end{array}$ & $\begin{array}{c}\text { Band 1 } \\
120-240 \mathrm{~Hz}\end{array}$ & $\begin{array}{c}\text { Band 2 } \\
240-360 \mathrm{~Hz}\end{array}$ & $\begin{array}{c}\text { Band 3 } \\
360-480 \mathrm{~Hz}\end{array}$ & $\begin{array}{c}\text { THBD } \\
\%\end{array}$ \\
\hline Ideal & 83.1785 & 3.0574 & 2.7787 & 1.9848 & 8.0372 \\
\hline $3^{\text {rd }} \mathrm{P}$ & 83.9581 & 4.5267 & 2.5578 & 1.8894 & 8.3579 \\
\hline $3^{\text {rd }} \mathrm{F}$ & 83.0131 & 6.0658 & 2.8638 & 2.1642 & 10.6785 \\
\hline $5^{\text {th }} \mathrm{P}$ & 83.4775 & 3.7258 & 2.7102 & 3.7499 & 9.5574 \\
\hline $5^{\text {th }} \mathrm{F}$ & 83.3600 & 4.6821 & 2.6780 & 4.2709 & 9.7901 \\
\hline $7^{\text {th }} \mathrm{P}$ & 82.0273 & 3.3288 & 5.7078 & 3.1611 & 10.4810 \\
\hline $7^{\text {th }} \mathrm{F}$ & 84.4958 & 2.8365 & 2.2612 & 1.7350 & 7.8111 \\
\hline $\mathrm{M} 7^{\text {th }}$ & 81.5016 & 4.8111 & 3.5052 & 3.0513 & 9.9604 \\
\hline $\mathrm{M} 3^{\text {rd }}$ & 80.6568 & 4.9145 & 2.8749 & 2.9174 & 9.6296 \\
\hline $\mathrm{M} \mathrm{5}^{\text {th }}$ & 81.0803 & 6.6234 & 2.6792 & 3.0491 & 10.6869 \\
\hline
\end{tabular}

Table 1 shows that the M5th, and 3rd flattened voltage distorted waveforms have the highest impact on the dc load current THBD. It will raise the third harmonic component in the dc load current. Table 2 shows that the 5 th peaked voltage distorted waveforms has the highest impact on the THBD for the transformer secondary current. It will raise the fifth harmonic component in the transformer secondary current. Table 3 shows that the 
$5^{\text {th }}$ peaked voltage distorted waveform has the highest impact on the magnetizing current. It will raise the fifth harmonic in the current. The difference between the harmonic responses of the transformer under sinusoidal and voltage supply distorted by $9^{\text {th }}$ and $11^{\text {th }}$ harmonics is small.

Table 8-2 WPT results for the transformer secondary current

\begin{tabular}{|c|c|c|c|c|c|}
\hline & $\begin{array}{c}\text { Band 0 } \\
0-120 \mathrm{~Hz}\end{array}$ & $\begin{array}{c}\text { Band 1 } \\
120-240 \mathrm{~Hz}\end{array}$ & $\begin{array}{c}\text { Band 2 } \\
240-360 \mathrm{~Hz}\end{array}$ & $\begin{array}{c}\text { Band 3 } \\
360-480 \mathrm{~Hz}\end{array}$ & $\begin{array}{c}\text { THBD } \\
\%\end{array}$ \\
\hline Ideal & 67.6432 & 7.1515 & 7.8811 & 13.0129 & 27.8975 \\
\hline $3^{\text {rd }} \mathrm{P}$ & 66.5970 & 9.6528 & 6.7138 & 13.5543 & 29.7245 \\
\hline $3^{\text {rd }} \mathrm{F}$ & 69.1658 & 7.3087 & 9.2830 & 11.4252 & 27.0932 \\
\hline $5^{\text {th }} \mathrm{P}$ & 66.3129 & 7.3275 & 5.8915 & 16.8675 & 32.2955 \\
\hline $5^{\text {th }} \mathrm{F}$ & 68.6885 & 6.7513 & 9.0769 & 9.6741 & 24.4384 \\
\hline $7^{\text {th }} \mathrm{P}$ & 66.7155 & 6.8632 & 6.9432 & 13.2562 & 25.6037 \\
\hline $7^{\text {th }} \mathrm{F}$ & 68.5685 & 7.2511 & 9.2865 & 11.9961 & 28.9540 \\
\hline $\mathrm{M} 7^{\text {th }}$ & 66.8332 & 6.5617 & 8.3409 & 11.9279 & 28.3109 \\
\hline $\mathrm{M} 3^{\text {rd }}$ & 65.512 & 8.3773 & 9.0746 & 9.978 & 28.9602 \\
\hline $\mathrm{M} \mathrm{5}^{\text {th }}$ & 67.5821 & 6.0805 & 7.6991 & 8.6662 & 24.4676 \\
\hline
\end{tabular}

In addition, from tables 8-1 to 8-3, one can conclude that the harmonic response of the transformer is mainly dependent on the phase angle of the voltage supply harmonics relative to the fundamental component, and a lesser extent on the magnitude of the harmonics components itself.

Harmonic characteristics of the transformer magnetizing current and load current are significantly different under nonsinusoidal supply voltage. The transformer harmonic response is mainly dependent on the phase angle of the voltage supply harmonics, and a lesser extent on the magnitude of the harmonics components itself. The effect of supply 
voltage harmonics gradually diminishes with the order of harmonics increase beyond the $11^{\text {th }}$ harmonic.

Table 8-3 WPT results for the transformer magnetizing current

\begin{tabular}{|c|c|c|c|c|c|}
\hline & $\begin{array}{c}\text { Band 0 } \\
0-120 \mathrm{~Hz}\end{array}$ & $\begin{array}{c}\text { Band 1 } \\
120-240 \mathrm{~Hz}\end{array}$ & $\begin{array}{c}\text { Band 2 } \\
240-360 \mathrm{~Hz}\end{array}$ & $\begin{array}{c}\text { Band 3 } \\
360-480 \mathrm{~Hz}\end{array}$ & $\begin{array}{c}\text { THBD } \\
\%\end{array}$ \\
\hline Ideal & 8.1013 & 0.5128 & 0.1435 & 0.1516 & 7.3384 \\
\hline $3^{\text {rd }} \mathrm{P}$ & 7.9791 & 0.7338 & 0.1413 & 0.1475 & 9.7665 \\
\hline $3^{\text {rd }} \mathrm{F}$ & 7.9773 & 0.5404 & 0.1492 & 0.2150 & 8.0942 \\
\hline $5^{\text {th }} \mathrm{P}$ & 7.9652 & 0.7004 & 0.1487 & 0.3182 & 10.7041 \\
\hline $5^{\text {th }} \mathrm{F}$ & 7.9990 & 0.5062 & 0.1742 & 0.3971 & 8.5790 \\
\hline $7^{\text {th }} \mathrm{P}$ & 8.0059 & 0.5007 & 0.1622 & 0.2015 & 7.3110 \\
\hline $7^{\text {th }} \mathrm{F}$ & 8.0059 & 0.5007 & 0.1622 & 0.2015 & 7.3110 \\
\hline $\mathrm{M}^{\text {th }}$ & 7.9609 & 0.6279 & 0.1901 & 0.2746 & 9.4166 \\
\hline${\mathrm{M} 3^{\text {rd }}}^{8}$ & 8.0957 & 0.5220 & 0.1930 & 0.4228 & 9.0463 \\
\hline $\mathrm{M} \mathrm{5}^{\text {th }}$ & 7.9295 & 0.5327 & 0.3315 & 0.2661 & 9.3641 \\
\hline
\end{tabular}




\section{Chapter 9: Rea-Time Distributed Simulation of Power Electronic Systems and}

\section{Drives}

\subsection{Introduction}

A drive system that consists of a motor controlled by a power electronics converter is a complex and nonlinear system. The major research areas in electrical drives are high level modeling of the integrated motor drive, new converter-inverter topologies, control algorithms, performance optimization, and fault tolerant controllers. Thus performing system-level testing which is one of the major subsystems in developing a complex product in a comprehensive and cost effective way, need real-time simulations.

In order to evaluate the interaction between the control system and the controlled target in real time a simulator in which the inputs and outputs of the tested control system can be connected to a real-time simulation of the target process is needed. This mean that the controller is connected across the controlled target, this method is known as hardware-in-the-loop (HIL) simulation. By using the HIL simulations we can evaluate different subsystem interaction and between the control algorithms and the controlled process in real time. In HIL simulations, we can connect certain hardware device to a simulated dynamic equivalent of an apparatus and run this system in real time. A particular advantage of this simulator is that it allows the gradual change from pure software simulation environment to mixed simulation environment by gradually integrating actual electrical and mechanical subsystems into the simulation loop. This is done be replacing a certain device model in the simulated system by the actual hardware. By utilizing HIL simulations in the design process can reduce the development cycles, 
cut the overall cost, prevent costly failures, and test the interaction between different subsystems comprehensively before integrating them together into one system.

With more detailed models used in the studies, the global system model built this way would be too complicated, this model will likely be very computationally intensive, slow, and time consuming. Also, the power electronic circuit simulation with the application of physical models of power semiconductor devices is very time-consuming and requires very strong computation tools. The multi-distributed modeling concept allows us to overcome this drawback. In this approach, the global model is to be distributed over several processors through communication link (TCP/IP, FireWire or Shared Memory).

Also, It's allows the subsystems (components or group of components) to be executed at different update rates, cycles can be freed up for executing the subsystem(s) that need to be updated faster. The multi-distribution modeling can involve different operating systems. Real time operating system (e.g. QNX or Linux) will reduce the simulation time requirement by a big percentage.

This chapter describes the real time with HIL simulator, and outlines its software and hardware subsystems.

\subsection{System Description}

The software and hardware tools used in the development and implementation of this real time with HIL simulator are discussed in this section. The overall system architecture is shown in Fig.9.1. This simulator provides us with an efficient way to evaluate different control algorithms, diagnose the incompatibilities of the motor with the drive system, predict the future performance of motor or power quality mitigating devices, or motor protection system, as well as design and evaluate digital control algorithms. 


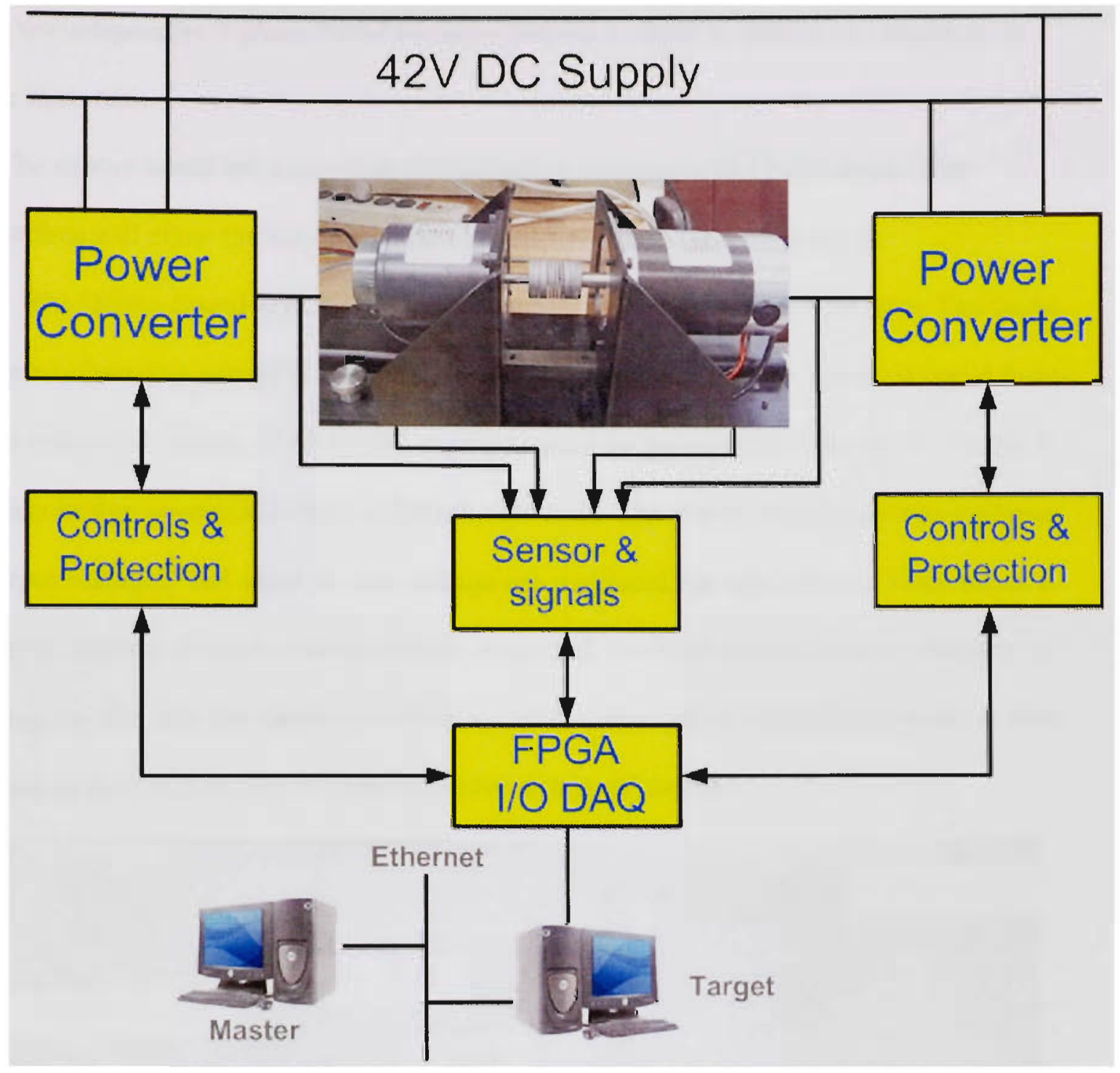

Fig.9.1 The real-time simulator architecture

\subsubsection{Drives board}

The drives board consists of two independent three phase inverters. Each inverter uses MOSFETs as switching devices [218]. The drives board can be used to conduct different drive experiments for both $\mathrm{AC}$ and $\mathrm{DC}$ machines.

The main features of the control board are [218]: 
- Two independent 3-phase PWM inverters that can be used to control two machines at the same time.

- The control board has a complete digital/analog interface with FPGA board. This interface will allow the implementation of real-time digital control systems.

The Drives Board is protected by overcurrent protection for each inverter. The faults can be cleared by using the"RESET" switch on the board or through a control signal from the command station. A $42 \mathrm{~V} \mathrm{DC}$ supply is used to provide the input power to the 3 phase bridge inverters through a flyback converter. The control board inverters currents, output voltages, and input dc bus voltage are measured through sensors. Only phase A and $\mathrm{B}$ currents of each inverter can be measured, the third current can be obtained by using the fact that the algebraic summation of the three phase currents in a balance three phase system is zero. Fig. 9.2 shows the drives boards' layout.

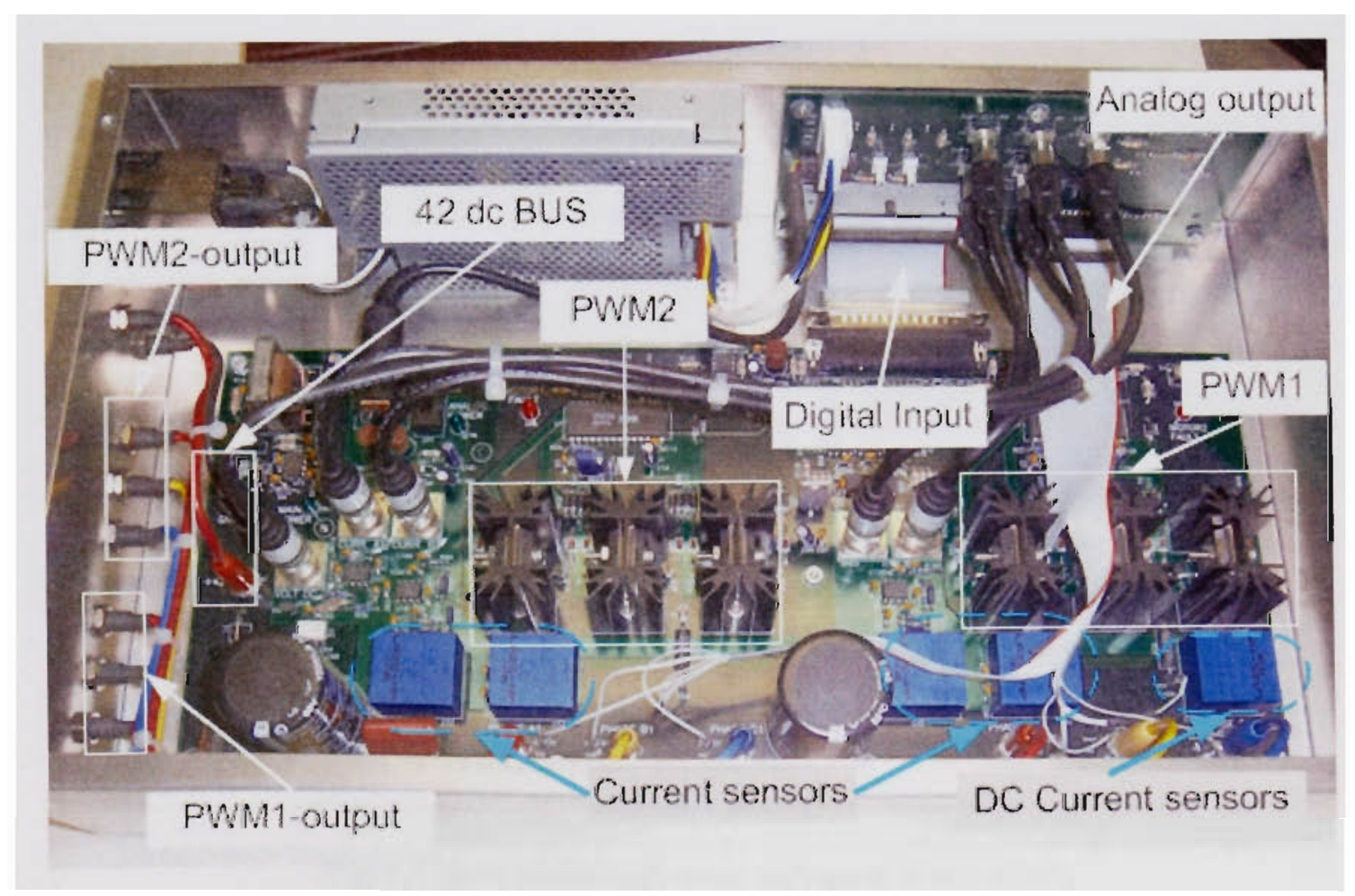

Fig.9.2 The drive board layout 


\subsubsection{Block diagram and schematic interface}

The real-time distributed simulation simulator is designed and optimized to run Simulink based models in real-time, with fixed time step solvers.

MATLAB is widely high level programming shell used in the simulation and analysis of dynamic systems and control analysis. MATLAB is a programming numerical analysis shell with add-on components called toolboxes. Simulink, a toolbox extension of MATLAB, is dynamic system simulation software which utilized a graphical programming language for building system models based on their mathematical description [25]. The Real Time Workshop (RTW) toolbox is capable of generating real time code for Simulink models. The real-time code is used to control a system in the HIL environment. The data generated by any of the system components, digital or hardware, can be displayed in real-time and/or saved for further analysis.

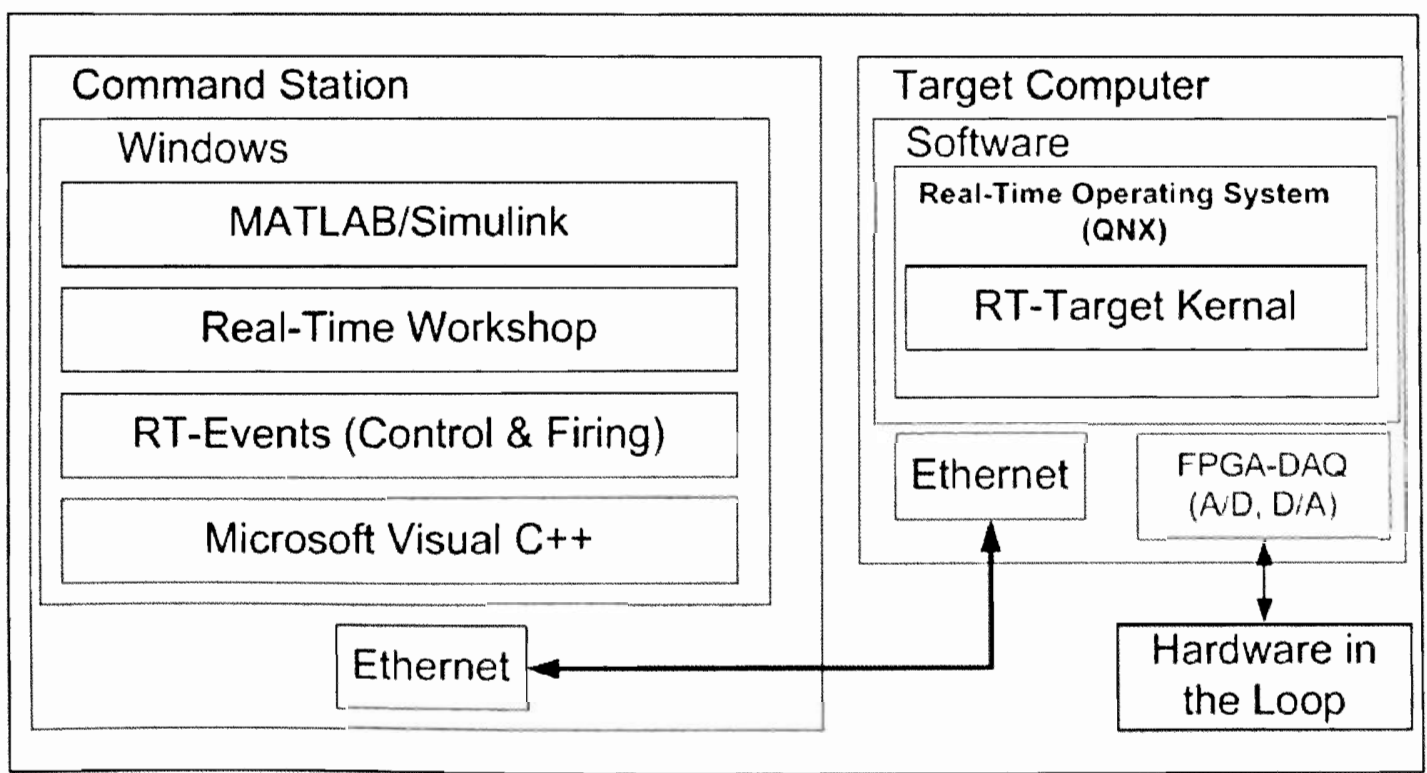

Fig.9.3 Host-target real-time so ftware architecture 
The simulator uses Simulink as interface shell for building the system simulator in block-diagram format. The block diagrams contained both the models for different system components, as well as the blocks that handle the I/O interface with the real hardware and the TCP/IP communication between the master and the target. The block diagram models are converted into C-code using RTW, and then transferred in to target machine for real-time simulation. Figure 9.3 shows the real time simulator software subsystems architecture.

\subsubsection{Distributed Computing Systems}

The ability to connect multiple computers through a communications network has existed for many years. Initially, computer-to-computer communication consisted of a small number of systems performing bulk file transfers. The 1980s brought the invention of high-speed local area networks, or LANs. A LAN allows hundreds of machines to be connected together.

New simulation approaches began to emerge, such as distributing a certain task between different computing nodes and send each node results to main node for display. This approach coordinates between the different computing nodes to achieve higher speed simulations using the communication link. A global model is divided into multi smaller subsystems; every subsystem is assigned to one of the computing node. In order to achieve real time simulations we need to use real-time operating systems (RTOS). These RTOS are those that guarantee that the system will respond in a predetermined amount of time.

We will utilize two different real time operating systems QNX and Linux. Both OS are designed for real time operation with communication abilities. 


\subsubsection{Data Acquisition}

A major subsystem in the real-time HIL simulator is the DAQ subsystem which is responsible for interfacing with real world hardware devices, controller, or sensors. The DAQ (I/O) provides the interface between the target computer and the HIL system.

High performance I/O Xilink Virtex-II pro Field Programmable Gate Arrays (FPGA) DAQ was used. This FPGA DAQ card can run HIL controlled motor inverter with ACside diode rectifier at time step as low as 40 microseconds.

The I/O interfaces are configured and controlled through Simulink custom blocks. The inputs and outputs interface to these blocks can be managed through Simulink directly, without writing low-level driver codes. The data collected will also be available directly for any subsystem inside the Simulink model for processing or for storing for further processing.

The RT shell generates automatically the I/O drivers and models code that handle and direct the flow data from or into the physical $\mathrm{I} / \mathrm{O}$ cards.

\subsubsection{Simulator Configuration}

The configuration of the real time simulator consists of following integrated subsystems:

- One or more target computation nodes (PC's) with real time operating system (e.g. QNX real-time operating system or Linux).

- Command or host station that represents the user interface with the system through which the user can control and change the system parameters. It also , coordinates the simulations between the targets, collects the results for display, and control the communication between the command station and the targets as well as the communication between the target PC's. 
- I/O's interface with the simulator (analog I/O, digital I/O, PWM I/O, encoders, etc). I/O's can be managed by dedicated processors.

The simulator transfers data and control signals between the host and the target PC through an Ethernet connection. This network connection allows the distribution of model computation between the system nodes and thus achieves small simulation time step size. Full control of the target application and computer using Simulink is provided to the user through the RT-shell.

RT-EVENTS are used to generate IGBT inverter signals (Pulses) for real-time simulation of electrical inverters with DC-stage [214]. 


\section{Chapter 10: Experimental Implementation}

\subsection{Introduction}

This chapter deals with the utilization of the distributed simulation environment to conduct system studies. First we will use the system to conduct pure software distributed simulations. Then we will use the hardware in the loop (HIL) approach to simulate and evaluate integrated motor drive system under different control algorithms.

\subsection{Distributed simulations}

In order to evaluate the benefits of the distributed simulation, we conducted a case study in which a PM phase variable model is controlled using vector based controller.

Figure 10.1 shows the Simulink model of the motor drive system.

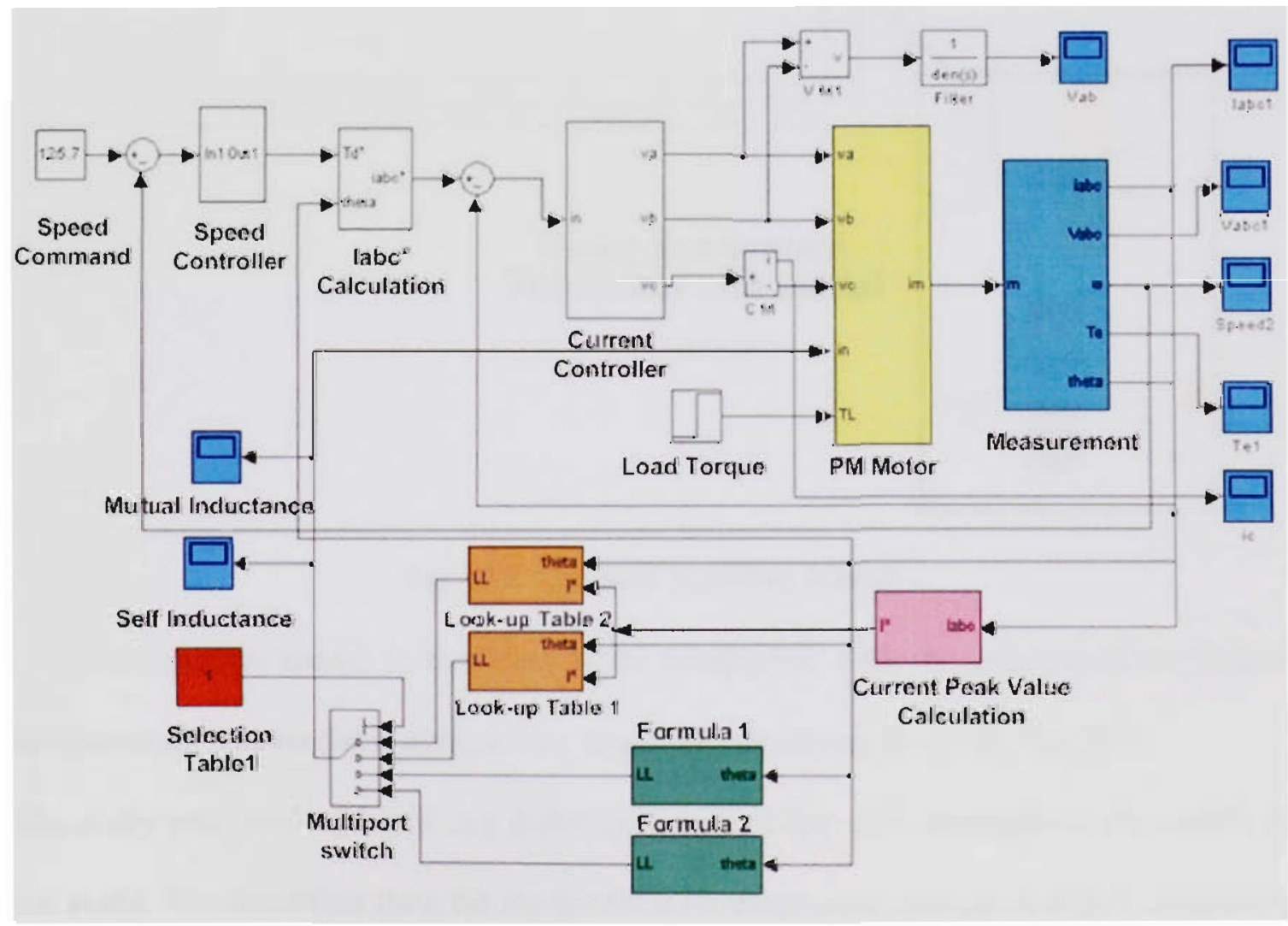

Fig.10.1 Simulink Model for PM synchronous vector control system 


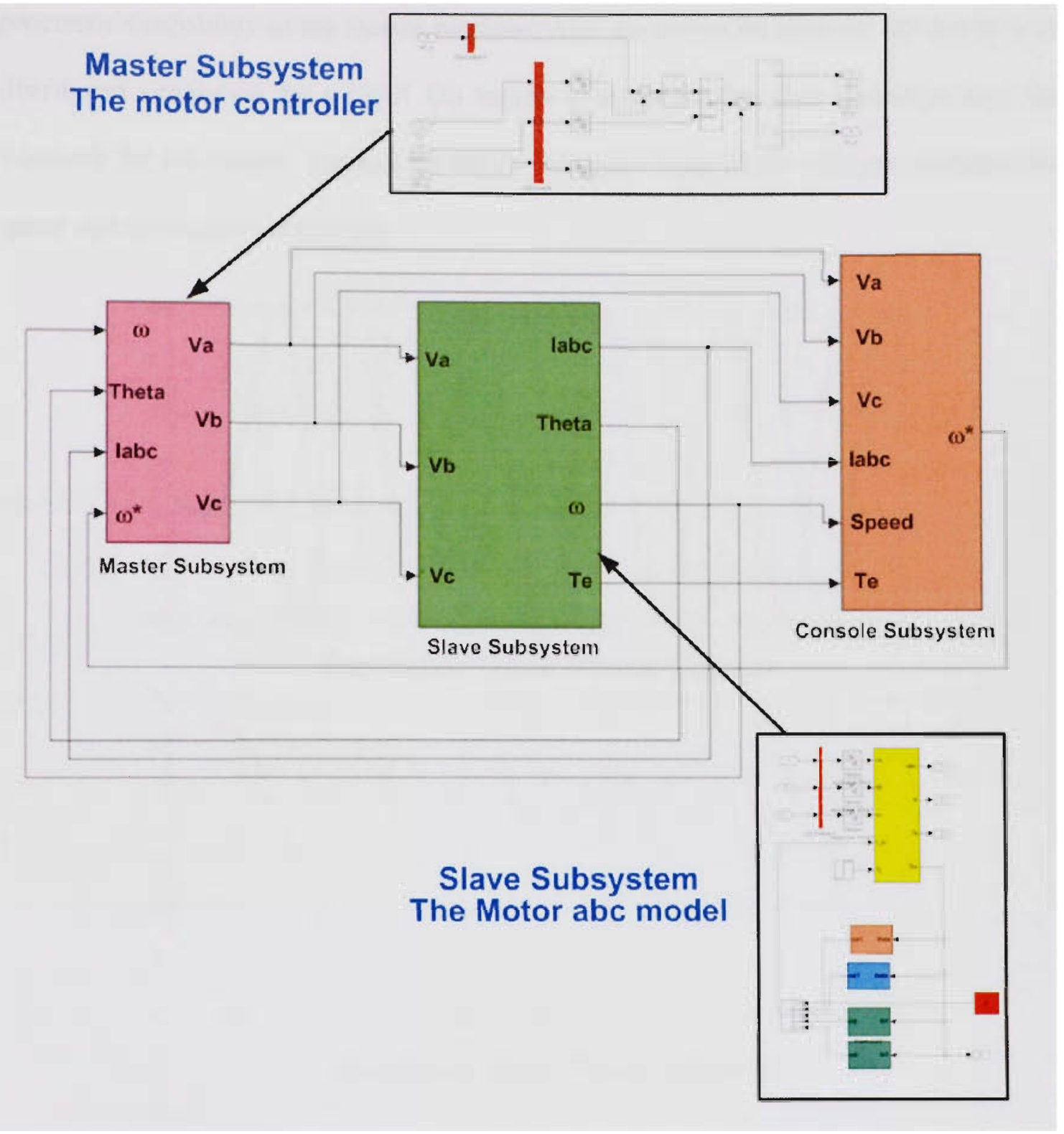

Fig.10.2 The final modified Model

The Simulink model is modified to be compatible with the distributed simulation environment. The model is divided into small subsystems as shown in Fig. 10.2.

The study was conduced utilizing different targets. Table 10.1 summarizes the results of the study. The execution time for the model with distributed simulation (QNX targets) is less than execution time for Simulink alone (almost half the time) despite the higher 
processor's capability of the master machine. Also the execution time for the model with distributed simulation for a QNX OS targets is much smaller than execution time for windows XP OS targets. Figure 10.3 shows the motor three phase voltages, currents, the speed and the torque versus time.
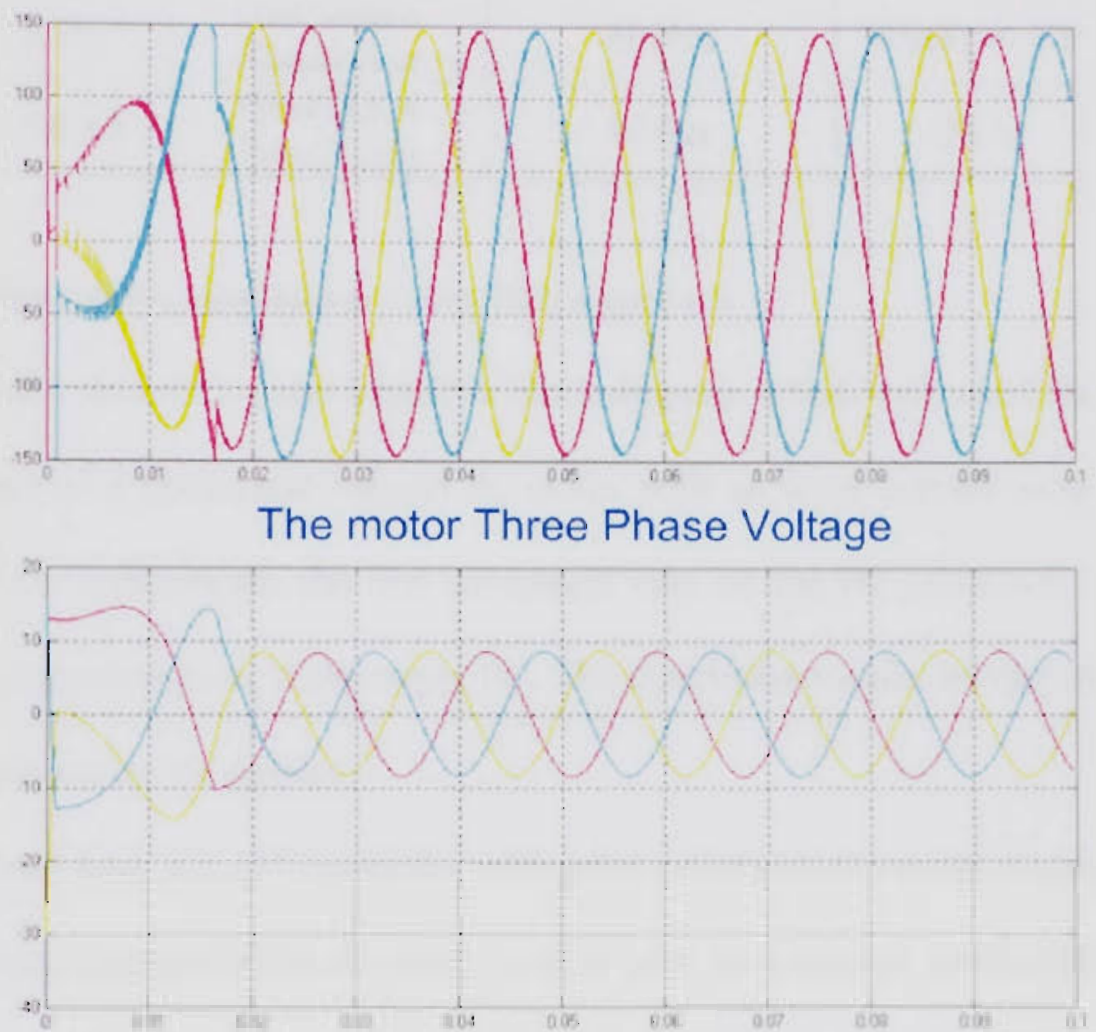

The motor three Phase currents

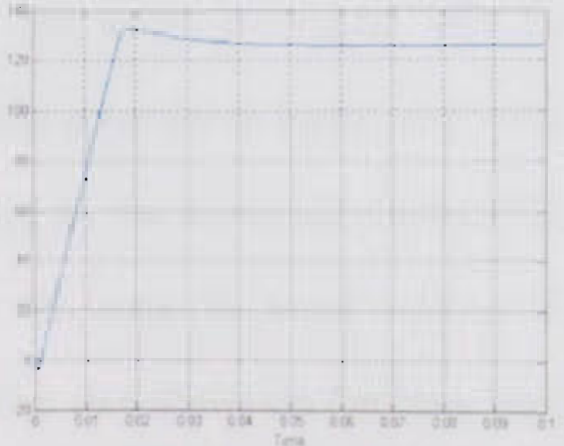

The motor speed

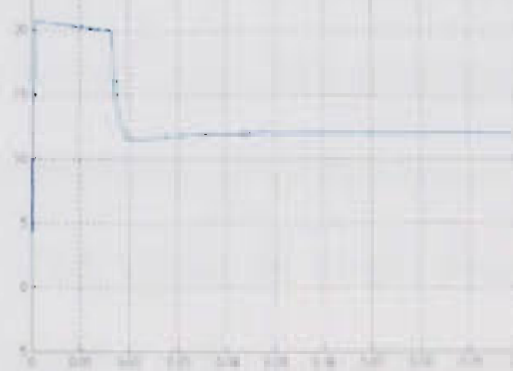

The motor Torque

Fig. 10.3 The motor voltage, current, speed and torque versus time 
Table 10.1 Comparison of Execution times for Simulink and distributed simulation

\begin{tabular}{|c|c|c|c|}
\hline Case & Method & $\begin{array}{c}\text { Execution Time } \\
\text { for 0.1 sec } \\
\text { simulation }\end{array}$ & Targets OS \\
\hline CASE 1 & $\begin{array}{c}\text { Simulink } \\
\text { (No simulation } \\
\text { distribution) }\end{array}$ & $20 \mathrm{sec}$ & -------- \\
\hline CASE 2 & $\begin{array}{c}\text { Distributed } \\
\text { Simulation }\end{array}$ & $40 \mathrm{Sec}$ & Windows XP \\
\hline CASE 3 & $\begin{array}{c}\text { Distributed } \\
\text { Simulation }\end{array}$ & $10 \mathrm{Sec}$ & QNX \\
\hline
\end{tabular}

\subsection{DC motor drive simulation using HIL approach}

Figure 10.4 shows the main Simulink block diagram which were used for closed-loop speed control of a permanent magnet dc motor with an inner current loop. The model consists of two subsystems, the first subsystem runs on the PC command station while the second subsystem runs at the target PC. The simulation results are then transferred to the command station for display.

The current loop has a PI controller with gains calculated to cancel the pole due to the electrical time constant of the dc motor, and to give the required bandwidth. The speed controller also has a PI controller designed to achieve a certain phase margin and bandwidth. The motor is coupled with another PMDC motor which functions as a generator. The generator is supplying a variable resistive load. The controller parameters are controlled by the command station GUI. A fixed-step sampling time of $80 \mu$ s was chosen for this setup to avoid overrun. Fig. 10.5 shows the block diagram of the controller. Fig. 10.6 shows the Simulink implementation of the controller block diagram.

The PWM signals are generated by RT-EVENTS and then send to a control board that runs the dc motor. 


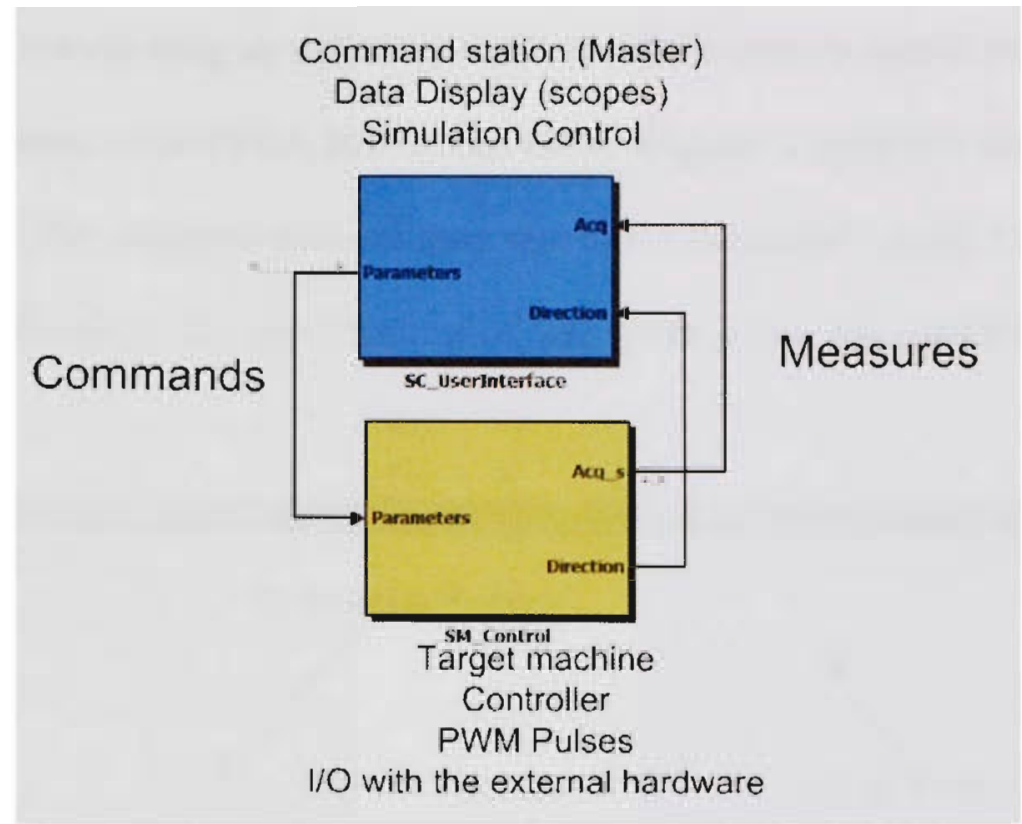

Fig.10.4 The Simulink block diagram for PMDC motor control

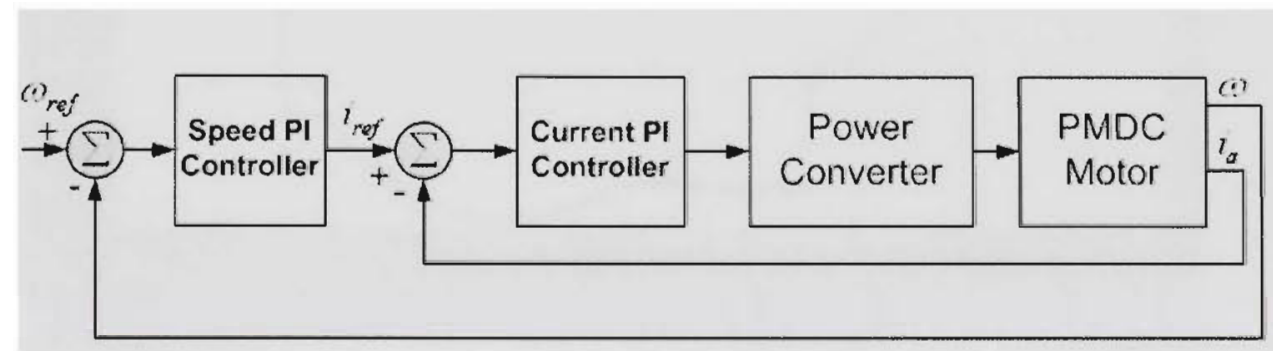

Fig.1 0.5 Block diagram of speed-current controller for an PMDC machine

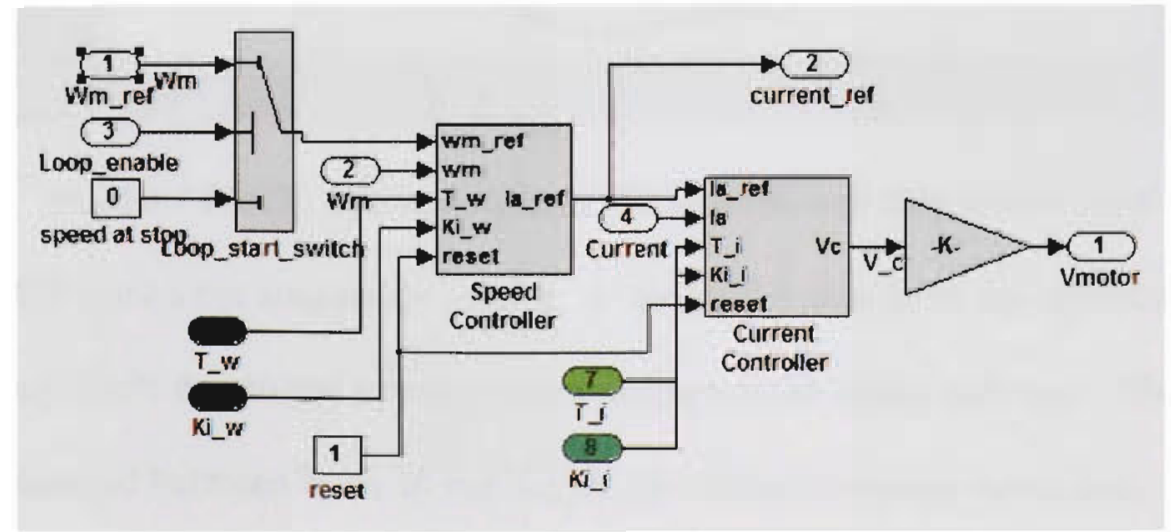

Fig.10.6 The controller Simulink block diagram details 
The speed is sensed using an optical encoder, and the current is sensed and fed to one of the ADC channels of the FPGA board. This block diagram is compiled and transferred to the target-PC. The collected data are then sent to the command station for display. A square pattern change in the speed reference was given using the graphical real time interface.
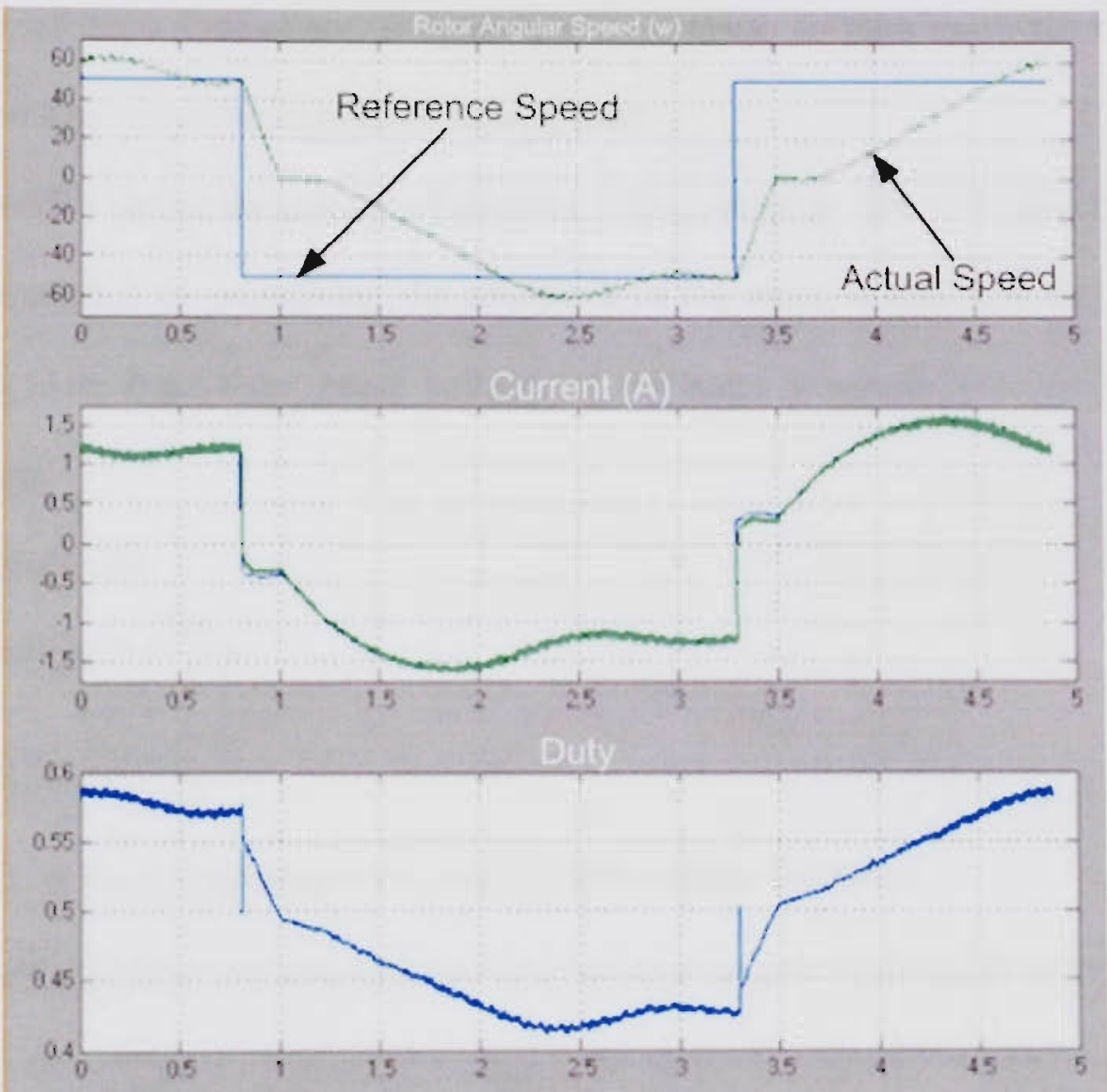

Fig.10.7 PMDC Machine speed, DC current, and duty versus time

Fig. 10.7 shows the simulation results. It shows the change in the speed, the current, and the duty cycle due to the square pattern change in the speed reference. The reference speed is changed between from $50 \mathrm{rad} / \mathrm{sec}$ to $-50 \mathrm{rad} / \mathrm{sec}$ (reversal operation). The results clearly show that the output of the controller follows the imposed reference. 


\subsection{Scalar based induction motor controller using HIL approach}

In scalar control schemes the phase relations between induction motor (IM) space vectors are not controlled during transient. The control scheme is based on steady state characteristics, which allows stabilization of the stator flux for different speed and torque values. In many industrial applications, the requirements related to the dynamic properties of drive control are of secondary importance. In such cases the open-loop constant voltage $/ \mathrm{Hz}$ control system is usually used.

Figure 10.8 shows the scheme of constant $\mathrm{V} / \mathrm{Hz}$ controller. The $42 \mathrm{~V}$ induction motor can be controlled by controlling the amplitude of the input voltage and the operating frequency. The input three phase voltages at the stator terminals have the following expressions.

$$
\begin{aligned}
& V_{A}=V_{m} \cos (2 \pi f t) \\
& V_{B}=V_{m} \cos \left(2 \pi f t-\frac{2 \pi}{3}\right) \\
& V_{C}=V_{m} \cos \left(2 \pi f t+\frac{2 \pi}{3}\right)
\end{aligned}
$$

Where $V_{m}$ is the voltage amplitude, and $f$ is the supply frequency

The control algorithm implies constant ratio between the amplitude of the voltage and the frequency $\left(k=V_{m} / f\right)$. Figure 10.8 shows the block diagram of the induction motor scalar controller. The control algorithm was implemented using the real time shell. The motor is coupled with another PMDC motor which functions as a generator. The generator is loaded with a variable resistor bank.

The controller parameters are controlled by the command station GUI. The V/f control algorithm is designed using Simulink and then implemented using the RT- 
simulator to run the $42 \mathrm{~V}$ induction motor. It was implemented on a 2 -node PC-cluster of 3.0 GHz P4 processors. Figure 10.9 shows the detail of the target PC block diagram.

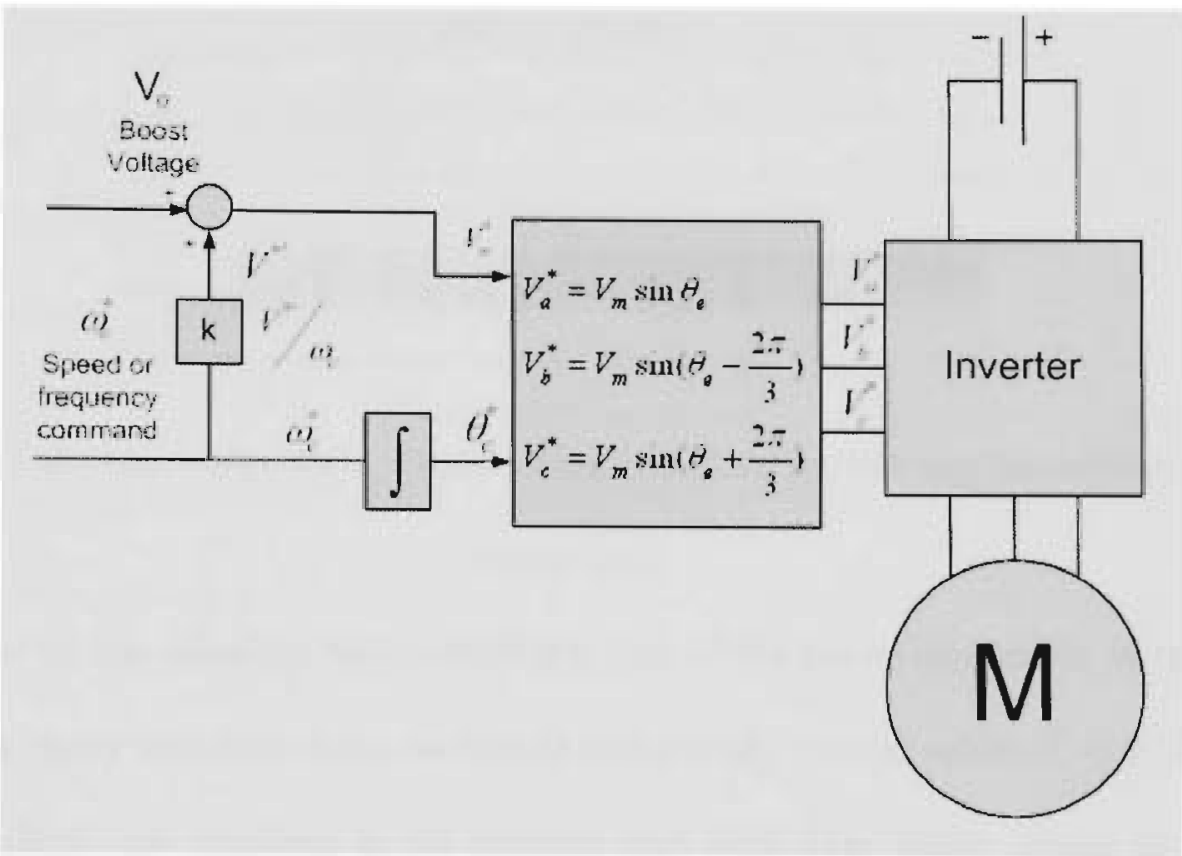

Fig.10.8 Block diagram of a scalar controller for an induction motor

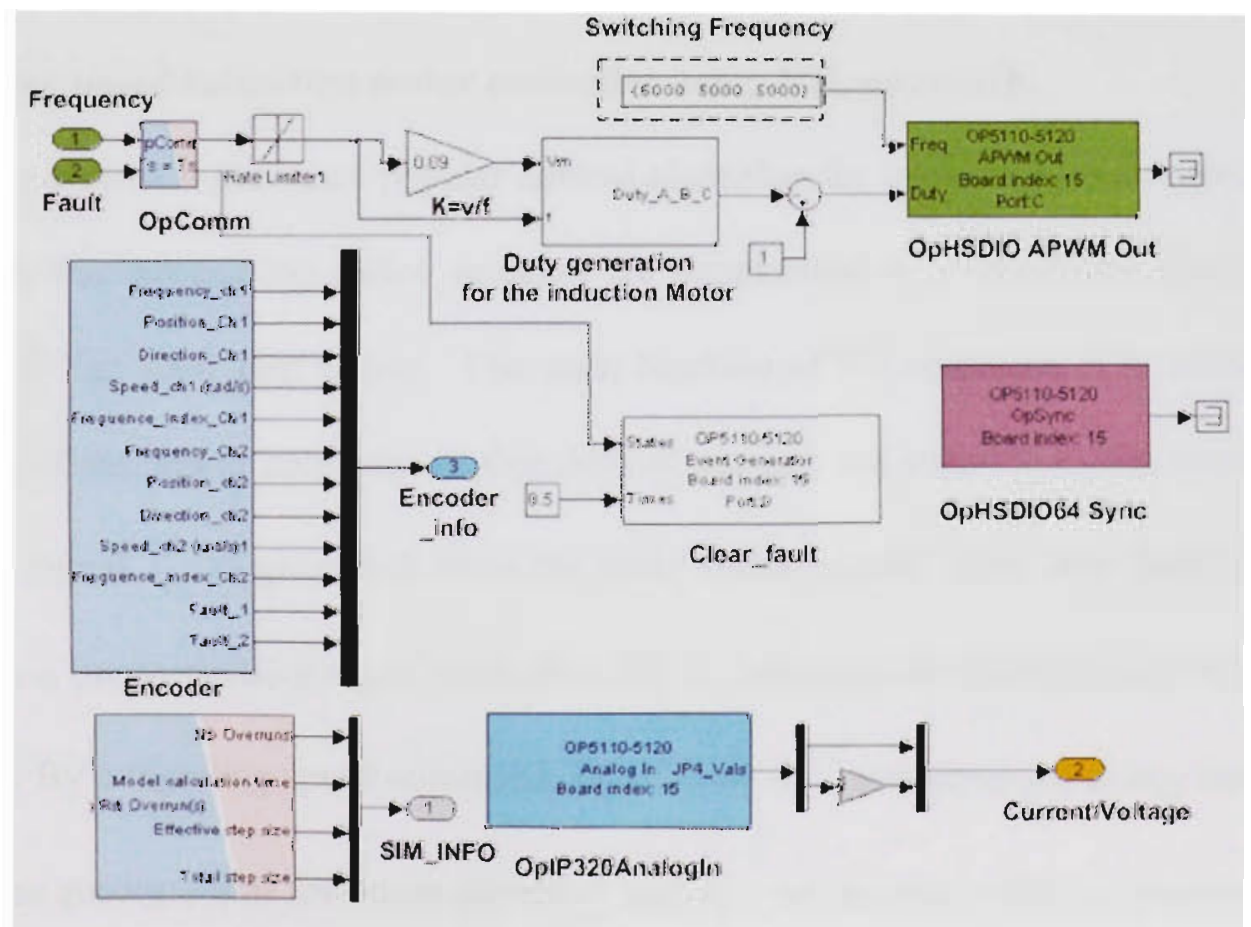

Fig.10.9 Inside the target PC Simulink block subsystem 


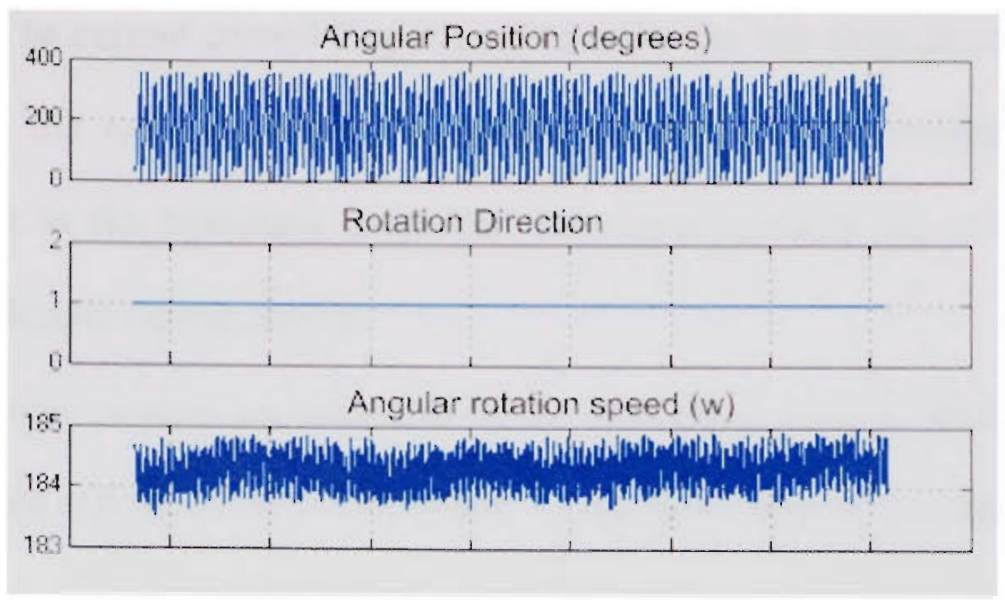

Fig.10.10 Induction motor theta, rotation direction, theta and the angular rotation speed versus time

The size of the sampling time constitutes one of the major constraints in real-time simulations, many tests have been conducted in this study and the value of was found to be the smallest one resulting in no overrun and with time factor equals to 1 . The simulation results are shown in Fig. 10.9.

\subsection{Vector based Induction motor controller using HIL approach}

Vector control is the most popular control algorithm for high performance induction motors. In this method the motor equation are transformed in a coordinate system that rotates with the rotor flux vector. The main function of the controller is to control the switching of the power converter so that desired currents are supplied to the motor. The reference signal $i_{q}^{*}$ is generated from the outer speed control loop. The speed control loop uses a proportional-integral controller (PI) to produce the quadrate-axis reference current $i_{q}^{*}$. By integrating speed command, rotor angle $\theta_{m}$ is determined at any instant of time. Once quadrate-axis reference current $i_{q}^{*}$ and $\theta_{m}$ are known, reference currents are calculated. To make sure motor is supplied with the desired currents, hysteresis current 
control is used. The current control loop is used to make the real three-phase currents of the motor equal the reference ones. The actual phase current is compared with its reference current in the hysteresis controller. Its output controls the switch position maintaining the desired output current.

The actual motor currents are measured by the Hall-effect sensors, which have good frequency response and fed to the FPGA board. As the motor neutral is isolated, only two phase currents are fed back to the board and the other phase current is calculated from them. The calibration of the current sensor is such that for $1 \mathrm{~A}$ current flowing through the current sensor, output is 0.5 volts. Q axis reference current is calculated from speed error signal by PI controller. Three phase reference currents are generated utilizing reference $\mathrm{q}$ axis current and rotor position angle which is obtained through encoder mounted on the shaft of the motor or through the estimator. Computed three phase reference currents are converted to three phase reference control voltages by the PI controller. The three phase reference control voltages are given as follows:

$$
\begin{aligned}
& V_{A}(t)=\hat{V}_{c} \sin (\omega t) \\
& V_{B}(t)=\hat{V}_{c} \sin \left(\omega t-\frac{2 \pi}{3}\right) \\
& V_{C}(t)=\hat{V}_{c} \sin \left(\omega t+\frac{2 \pi}{3}\right)
\end{aligned}
$$

Where: $\omega$ is the angular frequency of the control voltage and $\vec{V}_{c}$ is the amplitude. Assuming the magnitude of the triangular voltage equal to one, the duty ratios for three phases are generated according to the following equations. 


$$
\begin{aligned}
& d_{A}(t)=\frac{1}{2}+\frac{1}{2} \frac{\hat{V}_{c}}{\hat{V}_{t r i}} \sin (\omega t) \\
& d_{B}(t)=\frac{1}{2}+\frac{1}{2} \frac{\hat{V}_{c}}{\hat{V}_{t r i}} \sin \left(\omega t-\frac{2 \pi}{3}\right) \\
& d_{C}(t)=\frac{1}{2}+\frac{1}{2} \frac{\hat{V}_{c}}{2} \sin \left(\omega t+\frac{2 \pi}{3}\right)
\end{aligned}
$$

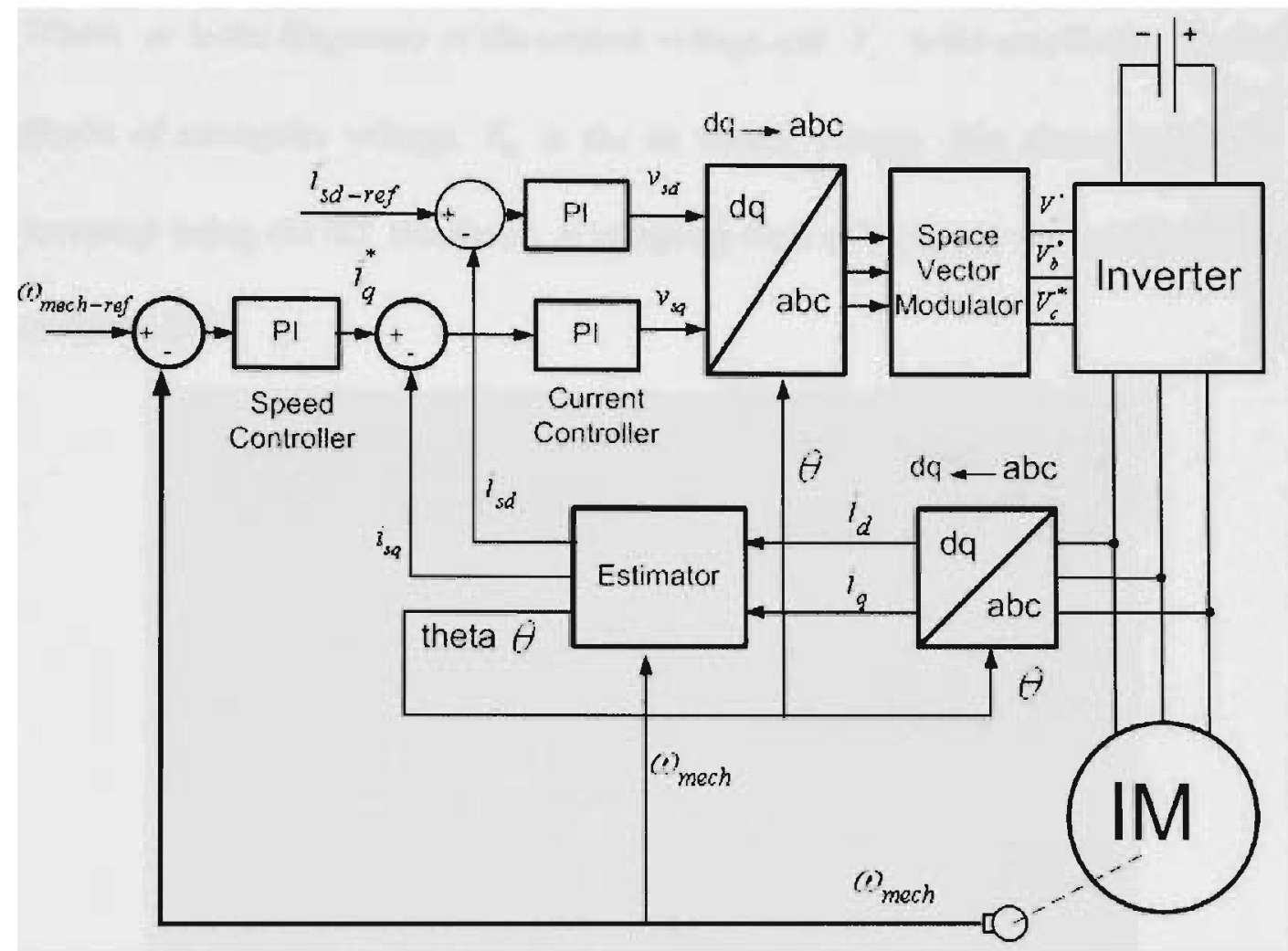

Fig.10.11 Block diagram of a vector controller for an induction motor

Figure 10.11 shows the block diagram of the vector controller. These duty ratios are fed to the PWM generating IC in drives board through the real time simulator. Inside the IC, the three phase control voltages are compared with the triangular waveform, the resultant of which is converted to six pulses by logical functions. The six pulses are fed to the MOSFET'S to generate three phase voltages whose average values are given by the following expressions: 


$$
\begin{aligned}
& \bar{V}_{A N}(t)=\frac{V_{d}}{2}+\frac{V_{d}}{2} \frac{\bar{V}_{c}}{\bar{V}_{t r i}} \sin (\omega t) \\
& \bar{V}_{B N}(t)=\frac{V_{d}}{2}+\frac{V_{d}}{2} \frac{\bar{V}_{c}}{\bar{V}_{t r i}} \sin \left(\omega t-\frac{2 \pi}{3}\right) \\
& \bar{V}_{C N}(t)=\frac{V_{d}}{2}+\frac{V_{d}}{2} \frac{\bar{V}_{c}}{\bar{V}_{t r i}} \sin \left(\omega t+\frac{2 \pi}{3}\right)
\end{aligned}
$$

Where $\omega$ is the frequency of the control voltage and $\hat{V}_{c}$ is the amplitude, $\hat{V}_{t r i}$ is the amplitude of triangular voltage, $V_{d}$ is the dc source voltage. The above algorithm is implemented using the RT simulator. A sampling time of $80 \mu \mathrm{sec}$ was used with time factor equals to 1 .
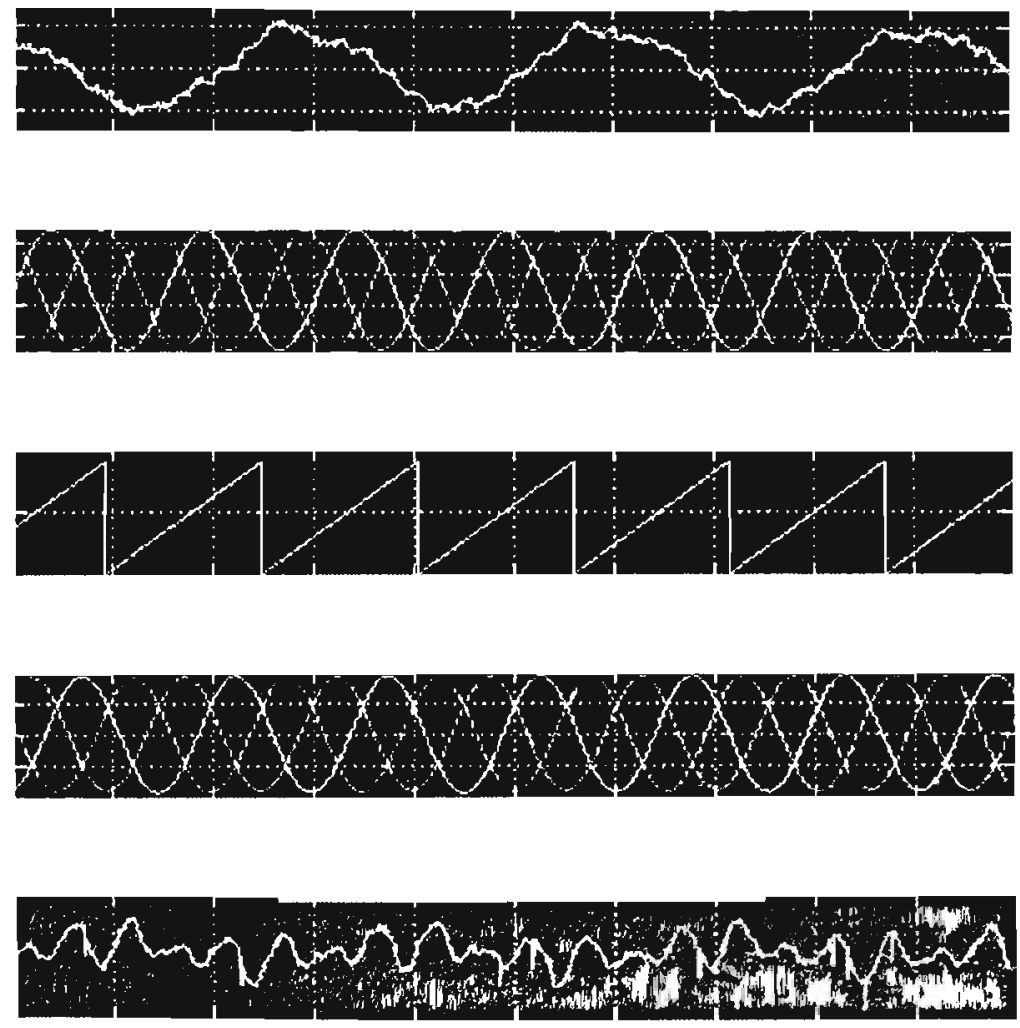

Fig.10.12 Induction motor speed, duty cycle, theta and the three phase currents versus time 
To make sure motor is supplied with the desired currents, hysteresis current control is used. The current control loop is used to make the real three-phase currents of the motor equal the reference ones. The actual phase current is compared with its reference current in the hysteresis controller.

Its output controls the switch position maintaining the desired output current. The speed loop controller has a bandwidth of $25 \mathrm{rad} / \mathrm{sec}$ and a phase margin of 60 degrees. The above algorithm is implemented using the RT simulator with hardware in the loop. A sampling time of $80 \mu s$ was used with time factor equals to 1. A snapshot of the simulation results displayed at the master machine is shown in Fig. 10.12.

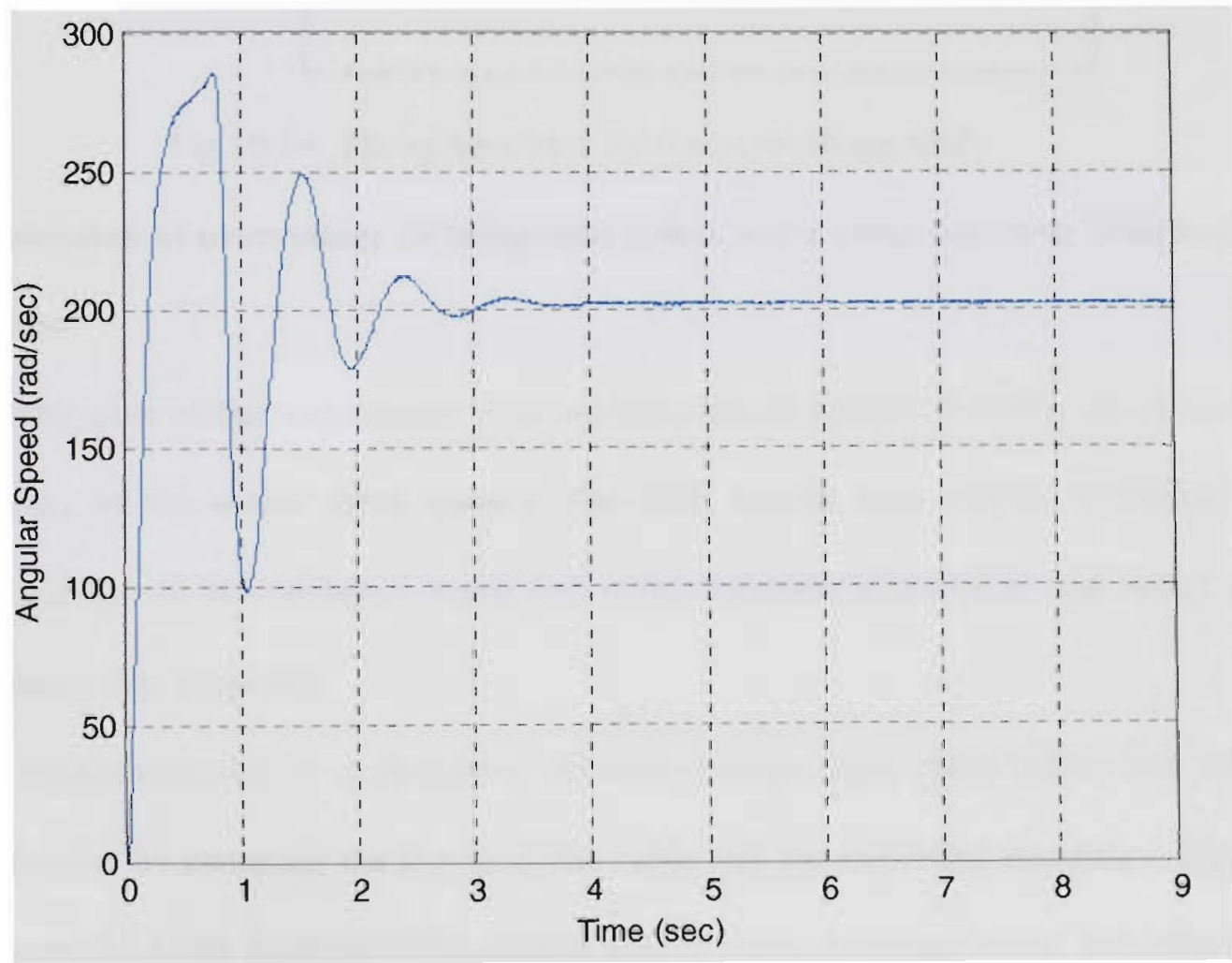

Fig. 10.13. Induction motor speed variation with time 


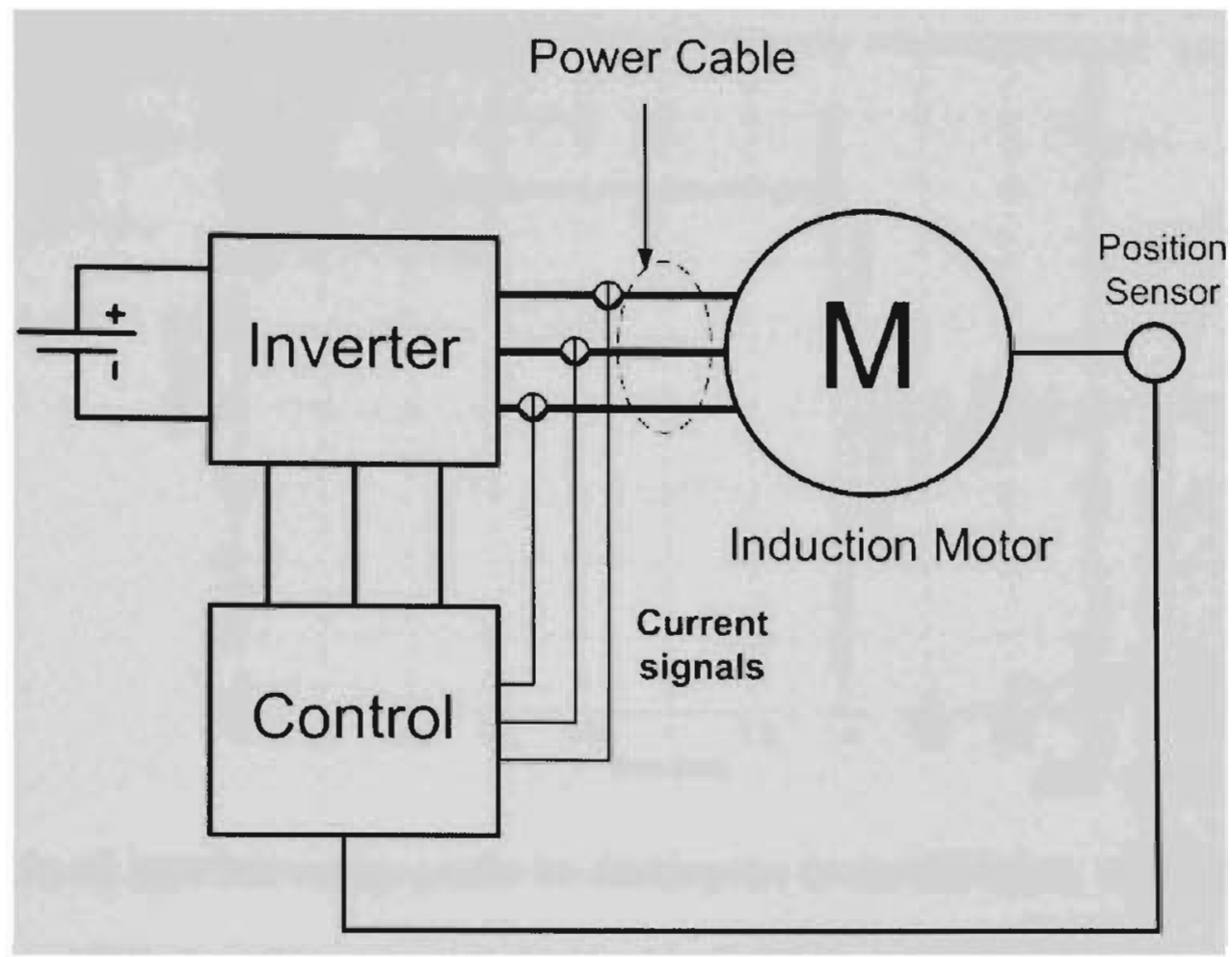

Fig. 10.14. The system used for the overvoltage study

10.6 Evaluation of overvoltage in integrated motor drive using real time distributed simulations

The objective of this experiment is to evaluate the interaction between the different components of the motor drive system. The HIL system was used to evaluate the overvoltage due to the reflected wave and common-mode phenomena and select the suitable switching frequency.

The induction motor is connected to inverter through three phase cable. The study was performed by changing the length of the cable and the switching frequency. Figure 10.14 shows the block diagram of the system used in the overvoltage study. The transient overvoltages results from the drive-motor-cable dynamic response to inverter pulse voltage. 


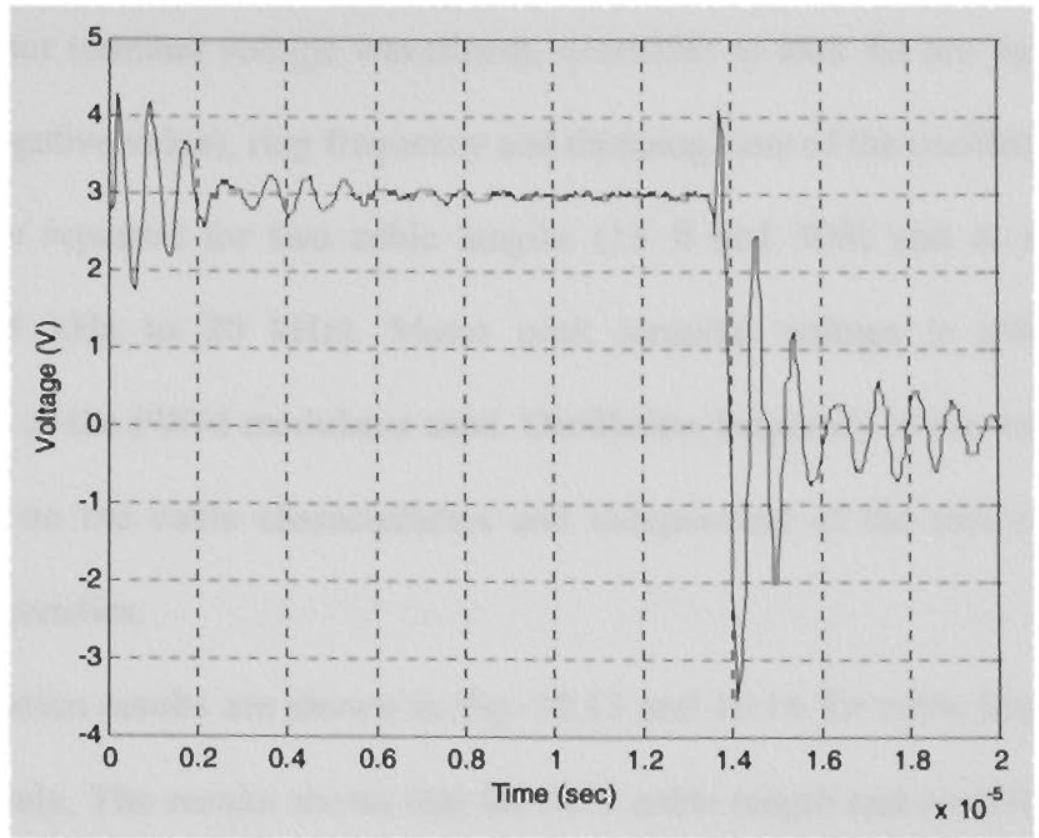

Fig.10.15. Line-line voltage profile for double pulse for the $42 \mathrm{~V}$ motor with $30 \mathrm{ft} 14$ AWG, 4-conductor braided shield cable @ 20kHz switching frequency

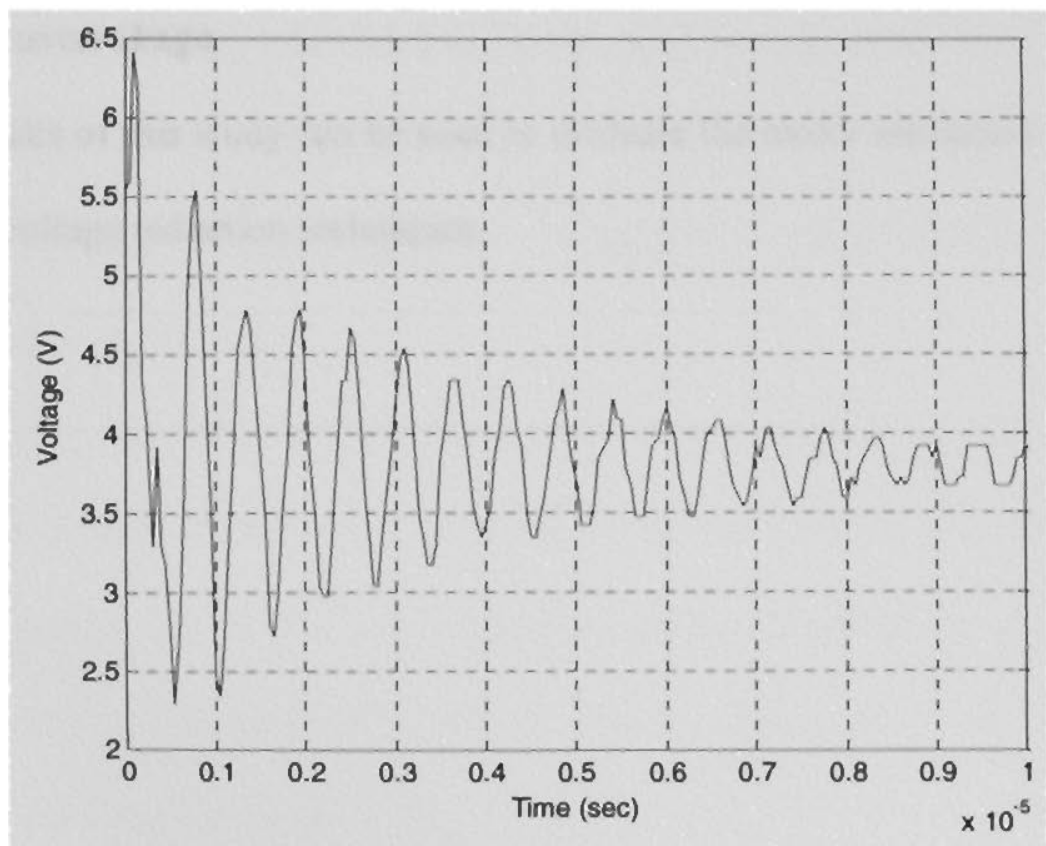

Fig.10.16. Line-line voltage profile for single pulse for the $42 \mathrm{~V}$ motor with $15 \mathrm{ft} 14$ AWG, 4-conductor braided shield cable@20kHz switching frequency 
At the motor terminal voltage waveforms, quantities to look for are peak magnitude (positive or negative value), ring frequency and damping time of the oscillation.

Tests were repeated for two cable lengths (15 ft and 30ft) and at several PWM frequencies $(5 \mathrm{kHz}$ to $20 \mathrm{kHz}$ ). Motor peak terminal voltage is affected by the characteristics of the PWM modulator used. Oscillation frequency at the motor terminals depends only on the cable characteristics and independent of the motor or the drive system characteristics.

The simulation results are shown in Fig. 10.15 and 10.16 for cable length of 15 and $30 \mathrm{ft}$ respectively. The results shows that for $30 \mathrm{ft}$ cable length and switching frequency of $20 \mathrm{khz}$ there will be $200 \%$ ( 2 p.u.) momentary overvoltage on the motor terminal, while for the $15 \mathrm{ft}$ cable length with $20 \mathrm{kHz}$ switching frequency there will be only $185 \%$ momentary overvoltage.

The results of this study can be used to evaluate the motor insulation system, and the motor overvoltage reduction techniques. 


\section{Chapter 11: Recommendations for future work}

Suggestions for further Studies that can be conducted as future additions or further studies are numerous. Such a future work and or additions that can be use to extend this work in the following ways:

- Use of the real time distributed environment to perform system level studies such as stability, and system reconfiguration.

- Additional developments to further refine and develop the models and parameter estimation methods covered in this dissertation.

- Modeling different types of non linear loads and their effect on the system overall harmonic behavior.

- Developing fault tolerant control algorithms for faulty equipments to minimize the damage and to keep them in service as long as possible. And then use the real time shell to evaluate the developed control algorithm.

- Developing detection algorithms for internal faults using the wavelet features obtained in this work.

- Studying the effect of replacing power electronics building block components on the system performance and plug and play components or subsystems.

- To include the dc bus system into the studies identified in this dissertation to examine the possibilities of adding alternate energy producing elements such as fuel cells and flywheel. 


\section{Chapter 12: Conclusion}

This dissertation presented the modeling and analysis of a typical shipboard power system using a real time distributed simulation environment. The Phase variable model is more accurate than normal d-q models. The distributed simulation concept was used to achieve real time simulation speed, which allows the connection of actual hardware directly in the simulation loop. The parameters used in building the different components models were obtained from coupled-FE analyses. This technique is significance because the FEA methodologies to extract high frequency parameters were shown to be accurate and can be used to eliminate costly manufacturing and testing. Different models were built for low and high frequency studies. These models were used to investigate different low and high frequency operational issues in the shipboard power system.

The Coupled Field- Circuit analysis was used to generate a database for internal faults. Modeling components with internal faults represent a challenge because of alternating nature of the magnetic field which will change the components parameters values. In practice, this can usually be obtained by measurements if a testing setup could be created. This dissertation would allow us to obtain these variations numerically.

A methodology for fault feature extraction and harmonic behavior evaluation for different shipboard power system components, using a wavelet based algorithm, was developed. The results show the ability of the wavelet based algorithm to extract harmonics and to capture the nonstationary waveform dynamics.

This modeling methodology can be utilized to evaluate and predicate the shipboard power system components future behavior in the design stage which will reduce the 
development cycles, cut overall cost, prevent failures, develop operational strategies and test the system exhaustively before integrating it into the system.

The distributed time simulation can be used to conduct real time simulations and can allow change gradually from pure software simulation environment to mixed simulation environment by integrating actual hardware into the simulation loop. 


\section{References}

[1] Naval ships technical Manual, "Electric Power Distribution Systems," Naval sea systems command, 1998.

[2] J. L. Ykeme, "Protective Devices in Navy Shipboard Electrical Power Systems," Naval Engineers Journal, May 1988, pp. 166-179.

[3] N. Doerry, J. Davis, "Integrated power system for marine applications," Naval Engineers Journal, 106, No. 3, May 1994, pp. 77-90.

[4] N. Doerry, H. Robey, J. Amy, and C. Petry, "Powering The Future with the Integrated Power System," Naval Engineers Journal 108, No. 3, May 1996, pp. 267-280.

[5] H. Zhang, K. L. Butler, N. D. R. Sarma, H. DoCarmo, S. Gopalakrishnan and A. Adediran, "Analysis of Tools for Simulation of Shipboard Electric Power Systems," Electric Power System Research, Vol. 58, Issue 2, June 2001, pp. 111-122.

[6] K.L. Butler, N.D.R. Sarma, C.A. Whitcomb, H.J. DoCarmo, and H. Zhang, "Shipboard Systems Deploy Automated Protection," IEEE Computer Application in Power 11, April 1998, pp. 31-36.

[7] H. Xiao, A. Adediran, and K. L. Butler, "Fault Scenario Studies Based On Geographical Information For Shipboard Power Systems," Proceedings of North American Power Symposium, Oct. 15-16, 2001, College Station, TX, pp. 436-442.

[8] A. Adediran, H. Xiao, and K. Butler, "Fault studies of an U.S. Naval shipboard Power System," Proceeding of North American Power Symposium, Waterloo, Canada, October 2000, pp. 18-25.

[9] H. Zhang, and K. Butler, "Simulation of Ungrounded Shipboard Power Systems in PSPICE," Proceedings of 1998 Midwest Symposium on Circuits and Systems, Notre Dame, IN, August 1998, pp. 58-62.

[10] A. T. Adediran, H. Xiao and K. L. Butler, "The Modeling and Performance Testing of a Shipboard Power System," Proceedings of 33rd annual Frontiers of Power Conference, October 2000, pp. VI-1 - VI-9.

[11] O. A Mohammed, S. Liu, and Z. Liu, "A Phase Variable Model of Brushless DC Motor Based on Physical FE Model and Its Coupling With External Circuits," IEEE Trans. on Magnetics, Vol. 41, No. 5, May 2005, pp. 1576-1579. 
[12] O. A Mohammed, S. Liu, and Z. Liu, "Physical Modeling of PM Synchronous Motors for Integrated Coupling with Machine Drives," IEEE Trans. on Magnetics, Vol. 41, No. 5, May 2005, pp. 1628-1631.

[13] O. A Mohammed, S. Liu, and Z. Liu, "A Phase Variable Model of Brushless DC Motor Based On Physical FE Model and Its Coupling with External Circuits," IEEE Trans. on Magnetics, Vol. 41, No. 5, May 2005, pp. 1576-1579.

[14] O. Mohammed, Z. Liu, and S. Liu, "A phase Variable PM Machine Model for Integrated Motor Drive Systems," in Proceedings of 2004 IEEE 35th Power Electronics Specialists Conference, PESC04, June 20-25, 2004, Aachen, Germany, pp. $4825-4830$.

[15] O. Mohammed, S. Liu, and Z. Liu, "Physical Modeling Study of Electrical Machinery," in CDROM of the Progress in Electromagnetics Research Symposium PIERS2004, March 28-31, 2004, Pisa, Italy, pp.763-766.

[16] O. Mohammed, Z. Liu, and S. Liu, "Physical Machine Models for Integrated Motor Drive Applications," in CDROM of the Electric Machines Technology Symposium EMTS2004, January 27-29, 2004, Philadelphia, USA.

[17] O. A. Mohammed, S. Liu, and Z. Liu, "FE-Based Physical Phase Variable Model of PM Synchronous Machines With Stator Winding Short Circuit Fault," to be presented at the 17th International Conference on Electric Machines, Sept. 2-5, 2006, Chania, Crete Island, Greece.

[18] O. A. Mohammed, S. Liu, Z. Liu, N. Abed, and S. Ganu, "Finite element based phase variable modeling of electrical machines for dynamic simulation of integrated drives," Electric Machines Technology Symposium 2006 (EMTS 2006), may 2224, Philadelphia, PA.

[19] O. A. Mohammed, S. Liu, and Z. Liu, "An FE-based physical phase variable model for PM synchronous machines including dynamic core losses," digest book of the 12th Biennial IEEE Conference on Electromagnetic Field Computation CEFC 2006 .

[20] Alternative Transients Program (ATP) Rule Book, Nov. 1995.

[21] Canadian/American EMTP User Group, "Alternative Transients Program (ATP) Rule book," 1995.

[22] MicroSim Corporation, PSpice A/D Simulator, Version 8.0, July 1997.

[23] Virtual Test Bed (University of South Carolina) Website, http://vtb.engr.sc.edu/ 
[24] MathWorks, Using Matlab, Inc., MA, 2006.

[25] MathWorks, Using Simulink, Inc., MA, 2006.

[26] MathWorks, Real Time Workshop User's Guide, Inc., MA, 2006

[27] MathWorks, SimPowerSystems User's Guide Version 4 , Inc., MA, 2006

[28] P.S. Shiakolas, and D. Piyabongkarn, "Development of a Real-Time Digital Control System with a Hardware-in-the-Loop Magnetic Levitation Device for Reinforcement of Controls Education," IEEE Trans. on education IEEE Trans, Vol. 46, Feb. 2003, pp. 79-87.

[29] A. B. J. Reece, and T.W. Preston, "Finite Element Methods in Electrical Power Engineering," Oxford University Press, 2000.

[30] I.A. Tsukerman, A. Konrad, G. Meunier, and J.C. Sabonnadiere, "Coupled FieldCircuit Problems: Trends and Accomplishments," IEEE Trans. on Magnetics, Vol. 29, No. 2, March 1993, pp. 1701-1704.

[31] G. Meunier, D. Shen, and J.L. Coulomb, "Modelisation of 2D and Axisymmetric Magnetodynamic Domain by the Finite Elements Method," IEEE Trans. on Magnetics, Vol. 24, No. 1, Jan. 1988, pp. 166-169.

[32] P. Lombard, and G. Meunier, "A General Purpose Method for Electric and Magnetic Combined Problems for 2D, Axisymmetric and Transient Systems," IEEE Trans. on Magnetics, Vol. 29, No. 2, March 1993, pp. 1737-1740.

[33] E. Vassent, G. Meunier, and A. Foggia, "Simulation of Induction Machines Using Complex Magnetodynamic Finite Element Method Coupled with the Circuit Equations," IEEE Trans. on Magnetics, Vol. 27, No. 5, Sept. 1991, pp. 4246-4249.

[34] E. Vassent, G. Meunier, A. Foggia, and G. Reyne, "Simulation of Induction Machine Operation Using a Step by Step Finite Element Method Coupled with the Circuits and Mechanical Equations," IEEE Trans. on Magnetics, Vol. 27, No. 6, Nov. 1991, pp. 5232-5234.

[35] F. Piriou, and A. Razek, "Coupling of Saturated Electromagnetic Systems to NonLinear Power Electronic Devices," IEEE Trans. on Magnetics, Vol. 24, No. 1, Jan. 1988, pp. 274-277.

[36] F. Piriou, and A. Razek, "Finite Element Analysis in Electromagnetic Systems Accounting for Electric Circuits," IEEE Trans. on Magnetics, Vol. 29, No. 2, Mar. 1993, pp. 1669-1675. 
[37] S. I. Nabeta, "Finite Element Simulations of Unbalanced Faults in a Synchronous Machine," IEEE Trans. on Magnetics, Vol. 32, No. 3, May 1996, pp. 1561-1564.

[38] H. Wang, and K. Butler, "Finite element analysis of internal winding faults in distribution transformers," IEEE Trans. on Power Delivery, Vol. 16, No. 4, Jul. 2001, pp. 422-428.

[39] F. de Leon and A. Semlyen, "Complete Transformer Model for Electromagnetic Transients," IEEE Trans. on Power Delivery, Vol.9, No.1, pp.231-239, January 1994.

[40] C. ONG, "Dynamic Simulation of Electric Machinery,” Prentice Hall, 1998.

[41] V. Brandwajn, H.W. Dommel and I.I. Dommel, "Matrix Representation of ThreePhase N-Winding Transformers for Steady-State and Transient Studies," IEEE Trans. on Power Apparatus and Systems, Vol.PAS-101, No.6, June 1982, pp.13691378.

[42] D.J. Wilcox, "Theory of Transformer Modeling using Modal Analysis," IEE proceeding, vol. 138, No.2, Mar. 1999, pp. 121-128.

[43] X. Chen, "A three Phase Multi-legged Transformer model in ATP using the directly-formed inductance matrix," IEEE Trans. on Power Delivery, Vol. 11, No. 3, Jul.1996, pp. 1554-1562.

[44] C.M. Artui, "Transient Simulation and Analysis of a three phase Step-up Transformer Following an out-of-phase Synchronization," IEEE Trans. on Power Delivery, Vol. 6, No. 1, Jan.1991, pp. 196-207.

[45] C. Saldaña and G. Calzolari, "Analysis of Core Type Transformer Models Based on the Principle of Duality in Electromagnetic Transients Studies," Proceedings of the International Conference on Power Systems Transients IPST'97, Seattle, USA, June 22-26, 1997.

[46] S. H. Khan, J. M. El-Shawish, L. Finkelstein, and K. T. V. Grattan, "Finite Element Modeling of Saturation and Eddy Current Effects in Commercial Variable Transformers," IEEE Trans. on Magnetics, Volume 37, Issue 4, July 2006, pp. 2783-2786.

[47] E. Dick and W. Walson, "Transformer Models for Transient studies Based on Field Measurements," IEEE Trans. on Power Apparatus and systems, Vol. PAS-100, No. 1, Jan 1981, pp. 409-418. 
[48] Q. Su, R. E. James, and D. Sutanto, "A z-transform model of transformers for the study of electromagnetic transients in power systems," IEEE Trans on Power Systems, vol. 5, Feb. 1990, pp. 27-33.

[49] S. Bouissou, F. Piriou, C. Kieny, and G. Tanneau, "Numerical simulation of a power transformer using 3D finite element method coupled to circuit equation," IEEE Trans. Magn., vol. 30, 1994, pp. 3224-3227.

[50] A. C. Delaiba, J. C. de Olivrira, J. R. Cardoso, and S. Y. Nabeta, "Behavior of Three Phase Transformers supplying non linear loads using time domain representation and Finite element analysis”. IEEE Trans. Magn., vol. 34, No. 5, Part: 1, Sept. 1998, pp. 3174-3177.

[51] P. T. M. Vaessen, "Transformer model for high frequencies," IEEE Trans. Power Delivery, vol. 3, Oct. 1988, pp. 1761-1768.

[52] IEEE/ANSI C57.110-1998, "IEEE recommendation practice for establishing transformer capability when supplying nonsinusoidal load currents," IEEE, New York.

[53] B. Gustavsen and A. Semlyen, "Rational approximation of frequency domain responses by vector fitting," IEEE Trans. Power Delivery, vol. 14, No. 3, July 1999, pp. 1052-1061.

[54] B. Gustavsen and A. Semlyen, "Application of vector fitting to the state equation representation of transformers for simulation of electromagnetic transients," IEEE Trans. Power Delivery, vol. 13, July 1998, pp. 834-842.

[55] B. Gustavsen, "Frequency dependent modeling of power transformers with ungrounded windings," IEEE Trans. Power Delivery, Vol. 19, No. 3, July 2004, pp. $1328-1334$.

[56] B. Gustavsen, "Application of vector fitting to high frequency transformer modeling," in Proc. International Conference on Power System Transients, IPST'2003, New Orleans, paper 9d-2, Sept 28 - Oct 2, 2003.

[57] B. Gustavsen, "Wide band modeling of power transformers," IEEE Trans. Power Delivery, vol. 19, No. 1, Jan. 2004, pp. 414-422.

[58] P. Bastard, P. Bertrand and M. Meunier, "A Transformer Model for Winding Fault Studies," IEEE Trans. on Power Delivery, Vo1.9, No.2, April 1994, pp.690-699.

[59] S. Santoso and P. Hofmann, "Power quality assessment via wavelet transform analysis," IEEE Trans. on Power Delivery, Vol. 11, No. 2, Apr. 1996, pp. 924-930. 
[60] D. C. Robertson, O. I. Camps, J. S. Mayer, and W. B. Gish, "Wavelets and electromagnetic power system transients," IEEE Trans. on Power Delivery, Vol. 11, No. 2, pp. 1050-1057, Apr. 1996.

[61] G. T. Heydt and A. W. Galli, "Transient power quality problems analyzed using wavelets," IEEE Trans. on Power Delivery, Vol. 12, No. 2, Apr. 1997, pp. 908-915.

[62] S. Santoso, W.M. Grady, E.J. Powers, J. Lamoree, and S.C. Bhatt, "Characterization of distribution power quality events with Fourier and wavelet transforms," IEEE Trans. Power Delivery, Vol. 15, 2000, pp. 247-254.

[63] W.K. Yoon and M.J. Devaney, "Power measurement using the wavelet transform," IEEE Trans. Instrum. Meas., Vol. 47, Oct. 1998, pp. 1205-1209.

[64] W.K. Yoon and M.J. Devaney, "Reactive Power measurement using the wavelet transform,” IEEE Trans. Instrum. Meas., Vol. 49, No. 2, April 2000, pp. 246-252.

[65] W. A. Wilkinson and M. D. Cox, "Discrete wavelet analysis of power system transients," IEEE Trans. Power Systems, Vol. 11, No. 4, Nov. 1996, pp. 20382044.

[66] K. Butler, and M. Bagriyanik "Characterization of Transients in Transformers Using Discrete Wavelet Transforms". IEEE Trans. Power Systems, Vol. 18, No. 2, May 2003, pp. 648-656.

[67] M. G. Morante and D. W. Nicoletti, "A wavelet-based differential transformer protection,” IEEE Trans. Power Delivery, Vol. 14, Oct. 1999, pp. 1351-1358.

[68] P. L. Mao and R. K. Aggarwal, "A wavelet transform based decision making logic method for discrimination between internal faults and inrush currents in power transformers," Electrical Power \& Energy Systems, Vol. 22, 2000, pp. 389-395.

[69] A.W. Galli and O. M. Nielsen, "Wavelet analysis for power system transients," IEEE Comput. Applicat. Power, Vol. 12, Jan. 1999, pp. 16-25.

[70] C. Parameswariah, and M. Cox, "Frequency Characteristics of Wavelets," IEEE Trans. Power Delivery, Vol. 17, July 2002, pp. 800-804.

[71] T. X. Zhu, "Effective Value Calculation in Wavelet Domain," IEEE Trans. Power Delivery, Vol. 19, No. 1, Jan. 2004, pp. 400-404.

[72] T. X. Zhu, S. K. Tso, and K. L. Lo "Wavelet-Based Fuzzy Reasoning Approach to Power-Quality Disturbance Recognition," IEEE Trans. Power Delivery, Vol. 19, No. 4, Oct. 2004, pp. 1928-1935. 
[73] T.B. Littler, and D. J. Morrow, "Wavelets for the Analysis and Compression of Power System Disturbances." IEEE Trans. Power Delivery, Vol. 14, No. 2, Apr. 1999 , pp. 358-364.

[74] Zwe-Lee Gaing, "Wavelet-Based Neural Network for Power Disturbance Recognition and Classification," IEEE Trans. Power Delivery, Vol. 19, No. 4, Oct. 2004, pp. 1560-1568.

[75] P. K. Dash, B. K. Panigrahi, D. K. Sahoo, and G. Panda, "Power Quality Disturbance Data Compression, Detection, and Classification Using Integrated Spline Wavelet and S-Transform," IEEE Trans. Power Delivery, Vol. 18, No. 2, Apr. 2003, pp. 595-600.

[76] M. V. Chilukuri, and P. K. Dash, "Multiresolution S-Transform-Based Fuzzy Recognition System for Power Quality Events," IEEE Trans. Power Delivery, Vol. 19, No. 1, Jan. 2004, pp. 323-330.

[77] E. Hamid, Z. Kawasaki, H. Yoshida, and H. Doi," Wavelet Analysis of Voltage Disturbances for Power Quality Applications," IEE of Japan. Vol.122, No.2, February 2002.

[78] H. A. Toliyat, G. Suresh and A. Abur, "Estimation of voltage distribution on the inverter fed random wound induction motor windings supplied through feeder cable," IEEE Trans. on Energy Conversion,Vol.14, No.4, Dec. 1999, pp. 976-981.

[79] G. Suresh, H. A. Toliyat, A. Abur, "Analysis of the effect of feeder cable on the stator winding voltage stress in a PWM induction motor drive," $23^{\text {rd }}$ Electrica1/Electronics Insulation Conference, Sept. 1997, pp.407-412.

[80] H. A. Toliyat, G. Suresh and A. Abur, "Simulation of voltage stress on the inverter fed induction motor winding supplying through feeder cable," IAS society general meeting, Oct. 1997, pp.143-150.

[81] G. Suresh, H.A. Toliyat, D.A. Rendusara, and P.N. Enjeti, "Predicting the Transient Effects of PWM Voltage Waveform on the Stator Winding of Random Wound Induction Motors", IEEE Trans. on Power Electronics, Vol. 14, No. 1, Jan. 1999, pp. 23-30.

[82] G. Grandi, D. Casadei, and U. Reggiani, "Common- and Differential-Mode HF Current Components in AC Motors Supplied by Voltage Source Inverters," IEEE Trans. Power Electronics, Vol. 19, No. 1, Jan. 2004, pp. 16-24. 
[83] A. F. Moreira, T. A. Lipo, G. Venkataramanan, S. Benet, "High frequency modeling for cable and induction motor overvoltage studies in long cable drives," IEEE Trans. on Industry Applications, Vol. 38, No.5, Sept/Oct. 2002, pp. 12971306.

[84] A.H. Bonnet and G.C. Soukup, "Cause and analysis of stator and rotor failures in three-phase squirrel-cage induction motors," IEEE Trans. Ind. Applicat., Vol. 28, July/Aug. 1992, pp. 921-937.

[85] H. Douglas, P. Pillay, A. Ziarani;" Current signature analysis of induction motor mechanical faults by wavelet packet decomposition," IEEE Trans. on Industrial Electronics, Vol. 50, No. 6, Dec. 2003, pp. 1217-1228.

[86] M. E. H. Benbouzid," A review of induction motors signature analysis as a medium for faults detection," IEEE Trans. on Industrial Electronics, Vol. 47, No. 5, Oct. 2000 , pp. $984-993$.

[87] G. H. Jang, , and S. J. Park, "Simulation of the Electromechanical Faults in a Single-Phase Squirrel Cage Induction Motor," IEEE Trans. on Magnetics, Vol. 39, No. 5, Sept. 2000, pp. 2618-2620.

[88] J. Watson, N. Paterson, D. Dorrell, "The Use of Finite Element Methods to Improve Techniques for the Early Detection of Faults in 3-phase Induction Motors," IEEE Trans. on Energy Conversion, Vol. 14, No. 3, Sept. 1999, pp. 655-660.

[89] J. Bangura and N. Demerdash," Diagnosis and Characterization of Effects of Broken Bars and Connectors in Squirrel-Cage Induction Motors by a TimeStepping Coupled Finite Element-State Space Modeling Approach," IEEE Trans. on Energy Conversion, Vol. 14, No. 4,Sept. 1999, pp. 1167-1175.

[90] J. Bangura and N. Demerdash," Effects of Broken Bars/End-Ring Connectors and Airgap Eccentricities on Ohmic and Core Losses of Induction Motors in ASD's Using a Coupled Finite Element-State Space Method," IEEE Trans. on Energy Conversion, Vol. 15, No. 1, Mar. 2000, pp. 40-47.

[91] H. Toliyat, and T. Lipo, “ Transient analysis of cage induction machines under stator, rotor bar and end ring faults," IEEE Trans. on Energy Conversion, Vol. 10, No. 2, Jun. 1995, pp. 241-247.

[92] J. Milimonfared, H. Kelk, S. Nandi, A. Minassians, and H. Toliyat, Senior Member, IEEE "A Novel Approach for Broken-Rotor-Bar Detection in Cage Induction Motors," IEEE Trans. on Industry Applications, Vol. 35, No. 5, sept./oct. 1999, pp. 1000-1006. 
[93] ST. Manolas, and J. Tegopoulos, "Analysis of Squirrel Cage Induction with Broken bars and Rings," IEEE Trans. on Energy Conversion, Vol. 14, No. 4, Jun. 1999, pp. 1300-1305.

[94] R. Fiser, and S. Ferkolj "Application of a Finite Element Method to Predict Damaged Induction Motor Performance," IEEE Trans. on Magnetics, Vol. 37, No. 5, Sept. 2001, pp. 2618-2620.

[95] T. Gammon, J. Matthews, "Arcing-fault models for low-voltage power systems," IEEE Annual Meeting 2000, May 2000, pp. 119-126.

[96] S. Sudhoff, , D. Aliprantis, B. Kuhn, and P. Chapman, "An Induction Machine Model for Predicting Inverter-Machine Interaction," IEEE Trans. on Energy Conversion, Vol. 17, No. 2, Jun. 2002, pp. 203-210.

[97] R. Dorf, “ The Electrical Engineering Handbook,” CRC Press, 2000

[98] R. Kaufman, and J.C. Page, "Arcing fault protection for low voltage power distribution systems - nature of the problem," IEEE Trans. on Power Apparatus and systems (AIEEE), June 1960.

[99] G. Melero, and F. Cabanas, "Electromagnetic torque harmonics for on-line interturn short circuits detection in squirrel cage induction motor," EPE99 Conference, Lausanne, 1999

[100] M. Say, "Performance and design of AC Machines," 3rd Edition, Pitman Publishing, 1974.

[101] J. A. Siegler, A. A. Sarkady, and C. Nemarich, "Motor Current Signal Analysis for Diagnosis of fault Conditions in Shipboard Equipment," Naval Engineers Journal, Jan 1995

[102] O. A. Mohammed, N.Y. Abed, and S. Liu " Investigation of the Harmonic Behavior of Three Phase Transformer Under Nonsinusoidal Operation Using Finite Element and Wavelet Packets," IEEE Trans. on Magnetics, Volume 42, Issue 4, April 2006, pp. 967-970

[103] E. Hamid, Z. Kawasaki, H. Yoshida, and H. Doi, "Wavelet Analysis of Voltage Disturbances for Power Quality Applications," IEE of Japan. Vol.122, No.2, February 2002

[104] O. A. Mohammed, N.Y. Abed, and S. Ganu "Modeling and characterization of induction motor internal faults using finite element and discrete wavelet transforms," IEEE Trans. on Magnetics, Vol. 42, Issue 4, Oct. 2006, pp. 3434 3436 
[105] O. A. Mohammed, Liu, Z., S. Liu, and N.Y. Abed, "Finite-Element-Based Nonlinear Physical Model of Iron-Core Transformers for Dynamic Simulations" IEEE Trans. on Magnetics, Vol. 42, Issue 10, April 2006, pp.1027-1030.

[106] O. A Mohammed, Z Liu, S. Liu, and N.Y. Abed "Electric machine operational modeling utilizing coupled electromagnetic and electric drives simulations," International Journal for Computation and Mathematics in Electrical Engineering (COMPEL), Vol. 24 (2), pp. 495-508.

[107] A. H. Chowdhury, W. M. Grady, and E. F. Fuchs, "An investigation of the harmonic characteristics of transformer excitation current under nonsinusoidal supply voltage," IEEE Trans. on Power Delivery, Vol. 14, No. 2, May 1996, pp. $450-458$.

[108] R. A. Rivas, and J. R. Martí, "Calculation of frequency-dependent parameters of arbitrarily-shaped power cables using the partial subconductor equivalent circuit method," IEEE Trans. on Power Delivery. Vol. 17, No. 4, October 2002, pp. 1085-1092.

[109] J. G. Reckleff, E. Fromholtz, R. Musil, S. Wenger, "Measurement of Fast Rise Time Transients Switching Large 13.2 KV Motors," IEEE power engineering society paper 87 WM $124-1$.

[110] H. Oraee, P.G. McLaren, "Surge Voltage Distribution in Line-End Coils of induction Motors,” IEEE Trans., Vol. PAS-104, No. 7, July 1985, pp. 1843-1848.

[111] D. H. Hwang, Y.J. Kim, S.W. Bae, D.H. Kim, and I.W. Lee, "Experimental Comparison of Insulation Systems for IGBT PWM Inverter-Fed Low-Voltage Induction Motors," 2002 33rd Annual IEEE PESC Proceedings, Australia, Vol. 2, June 23-27, 2002, pp. 973-978.

[112] L. Gubbala, A. von Jouanne, P. Enjeti, C. Singh, H. Toliyat, "Voltage Distribution in the Windings of an AC Motor Subjected to High dv/dt PWM Voltages," Proc. of PESC Conference, June 1995, pp. 579-585.

[113] G. Grandi, D. Casadei, U. Reggiani, "Equivalent Circuit of Mush Wound AC Windings For High Frequency Analysis," ISIE 1997, Portugal, pp. 201-206.

[114] A.Consoli, G. Oriti, A. Testa, A.L. Julian, "Induction Motor Modeling for Common Mode and Differential Mode Emission Evaluation," Proceedings of IAS Conference, October 1996, Vol. 1, pp. 595-599.

[115] G. Grandi, D. Casadei, A. Massarini, "High Frequency Lumped Parameter Model for AC Motor Windings,” EPE 1997, Trondheim, Norwegen, pp. 2578-2583. 
[116] J. L. Guardado, and K.J. Comick, "Calculation of Machine Winding Electrical Parameters at High Frequency for Switching Transient," IEEE Trans. on Energy Conversion, Vol. 11, No. 1, Mar. 1996, pp. 33-40.

[117] Boglietti, E. Carpaneto, "Induction Motor High Frequency Model," Conf. Rec. IEEE-IAS Annual Meeting, 1999, pp. 1551-1558.

[118] Boglietti, A. Cavagnino, M. lazzari, "Experimental High Frequency Parameter Identification of AC Electrical Motors," IEMDC 2005.

[119] S.D. Sudhoff, J.L. Tichenor, J.L. Drewniak, "Wide-Bandwidth Multi-Resolutional Analysis of a Surface-Mounted PM Synchronous Machine," IEEE Trans. Energy Conversion, Vol. 14, No. 4, Dec. 1999, pp. 1011-1018.

[120] S.D. Sudhoff, K.A, Coraine, and H.J, Hegner, "A Flux-Weakening Strategy for Current-Regulated Surface-Mounted Permanent Magnet Machine Drives," IEEE Trans, on EC, Vol. 10, No. 3, Sept. 1995, pp. 431-437.

[121] K.A. Corzine, S.D. Sudhoff, "A Hybrid Observer for High Performance brushless DC Drives," IEEE Trans. Energy Conversion, Vol. 11, No. 2, June 19, pp. 318323.

[122] S. Chen, T. A. Lipo and D. Fitzgerald, "Source of Induction Motor Bearing Currents Caused by PWM Inverters," IEEE Trans. Energy Conversion, Vol.11, No. 1, Mar. 1996, pp. 25-32.

[123] S. Chen, T. A. Lipo and D. Fitzgerald, "Modeling of Motor Bearing Currents in PWM Inverter Drives," IEEE Trans. on Industry Application., Vol. 32, No. 6, Nov./Dec. 1996, pp. 1365-1370.

[124] F. de Leon and A. Semlyen, "Reduced order model for transformer transients," IEEE Trans. on Power Delivery, Vol.7, No.1, Jan. 1992, pp.361-369.

[125] H. Zhu, J. Lai, Y. Tang, A. Hefner, D. Berning, and C. Chen, "Analysis of Conducted EMI Emissions From PWM Inverter Based On Empirical Models And Comparative Experiments," IEEE PESC June 1999, pp. 111-123.

[126] E. Person, "Transient Effects in Applications of PWM Inverters to Induction Motors," IEEE Trans. on Industry Applications, Vol.28, No.5, Sep/Oct 1992, pp.1095-1101.

[127] H. Bonnett, "Analysis Of Impact of Pulse Width Modulated Inverter Voltage Waveforms On AC Induction Motors," IEEE Trans. on Industry Applications, Vol. 32, No.2, Mar/Apr 1996, pp.386-392. 
[128] R. Kerkman, D. Leggate, and G. Skibinski, “Interaction Of Drive Modulation and Cable Parameters On AC Motor Transients," Proceedings of 31 st IEEE Industry Applications Society Conference (IAS'96), Vol.1, pp.143-152, San Diego, CA, USA, 1996.

[129] F. Moreira, T. A. Lipo, G. Venkataramanan, and S. Benet, "Modeling And Evaluation Of Dv/Dt Filters For AC Drives With High Switching Speed," Proceedings of 9th European conference of power electronics and applications (EPE'01) Aug.27-29, Graz, Austria, 2001.

[130] L. Gubbala, A. von Jouanne, P. N. Enjeti, C. Singh, and H. A. Toliyat, "Voltage Distribution In The Windings Of An AC Motor Subjected To High dv/dt PWM Voltages," in IEEEPESC Conf. Proc, 1995, pp. 579-585.

[131] G. Skibinski, "Design Methodology of a Cable Terminator to Reduce Reflected Voltage on AC Motors," in IEEE IAS Conf. Proc, 1996, pp.153-161.

[132] D. B. Hyypio, "Simulation of cable and winding response to steep-fronted voltage waves," Proceedings of IEEE IAS Conf., 1995, pp. 800-806.

[133] A. Von Jouanne, D. Rendusara, and P. N. Enjeti, "Filtering techniques to minimize the effect of long motor leads on PWM inverter-fed AC motor drive systems." IEEE Trans. on Industry Applications, Vol. 32, No. 4, July/Aug.1996, pp.919-926.

[134] C.J. Melhorn and L. Tang, "Transient effects of PWM drives on induction motors," Proceedings of IEEE/I\&CPS Conf. Rec, May 1995, pp. 1-7.

[135] R. J. Kerkman, D. Leggate, D. Schlegel, and G. Skibinski, "PWM inverters and their influence on motor over-voltages," Proceedings of IEEE APEC Conf. Rec, 1997, pp.103-113.

[136] G. Skibinski, D. Leggate, and R. J. Kerkman, "Cable characteristics and their influence on motor over-voltages," Proceedings of IEEE APEC Conf. Rec, 1997, pp. 114-121.

[137] A. Hussain and G. Joss, "Modeling and simulation of traveling waves in induction motor drives," Proceedings of IEEE APEC Conf. Rec, 1997, pp. 128-134.

[138] K.J. Cornick and T.R. Thompson, "Steep fronted switching voltage transients and their distribution in motor windings; Part 1: System measurements of steep fronted switching voltage transients," IEE proceedings, Vol. 129, part B, March 1982, pp.45-55. 
[139] K. Gupta, B.A. Lloyd, G.C. Stone, S. Campbell, D. Sharma, N. Nilsson, "Turn insulation capability of large AC motors; part 1: Surge monitoring," IEEE paper No. 87WM199-3, 1987.

[140] D.H. Hwang, J.W. Jeon, Y.J. Kim, S.W. Bae, D.H. Kim, M.H. Kim, and I.W. Lee, "Evaluation of Insulation Systems for IGBT PWM Inverter-Fed LowVoltage Induction Motors", Proceedings of the 2002 IEEE International Symposium on Industrial Electronics, L'Aquila, Italy, Vol. 4, July 8-11,2002, pp. 1154-1159.

[141] J.M. Bentley and P.J. Link, "Evaluation of Motor Power Cables for PWM AC Drives", IEEE Trans. on Industry Applications, Vol. 33, No. 2, Mar./Apr. 1997, pp. 342-358.

[142] D.H. Hwang, Y.J. Kim, S.W. Bae, D.H. Kim, and I.W. Lee, "A Study on Voltage Distribution in Stator Winding of Low-Voltage Induction Motor Driven by IGBT PWM Inverter", Proceedings of the ICEE, Korea, Vol. 2, July 7-11,2002, pp. 1006-1010.

[143] A. Von Jounne, and P. N. Enjeti, "Design consideration for an inverter output filter to mitigate the effects of long motor leads in ASD applications," IEEE Trans. on Industry Applications, Vol.33, No.5, Sep/Oct. 1997, pp.1138-1145.

[144] S. J. Kim and S. K. Sul, "A novel filter design for suppression of high voltage gradient in voltage- fed PWM inverter," Proceedings of 12th IEEE annual Applied Power Electronics Conference and Exposition (APEC 1997), Vol. 1, Atlanta, USA, 1997, pp.122-127.

[145] G. Skibinski, R. Kerkman, D. Leggate, J. Pankau, and D. Schlegel, "Reflected wave modeling techniques for PWM AC motor drives," Proceedings of 13th IEEE Annual Applied Power Electronics Conference and Exposition," (APEC'98), Vol.2, CA, USA, 1998, pp.1021-1029.

[146] M.T. Wright, S.J. Yang, K. McLeay, "General theory of fast-fronted interturn voltage distribution in electrical machine windings," IEE Proc. Part. B, Vol. 130, No. 4, July 1983, pp. 245-256.

[147] P. G. McLaren and H. Oraee, "Multiconductor transmission line theory model for the line-end coil of large ac machines," Proc Inst. Elect. Eng., vol. 132, Part B, May 1985, pp. 149-156.

[148] J. L. Guardado, K.J. Cornick, "A Computer Model for Calculating Steep-Fronted Surge Distribution in Machine Windings," IEEE Trans, Vol.EC-4, No. 1, March 1989, pp. 95-101. 
[149] Y. Tang, "Analysis of steep-fronted voltage distribution and turn insulation failure in inverter fed ac motor," in IEEE IAS Conf. Rec, 1997, pp. 509-516.

[150] D.H. Hwang, K.C. Lee, Y.J. Kim et al., " Voltage stresses on stator windings of induction motors driven by IGBT PWM inverter," 38th IAS annual meeting, Oct. 2003, vol.1, pp. 439-444.

[151] F. Piriou, and A. Razek, "Simulation of electromagnetic systems by coupling of magnetic and electric equations," Mathematics and Computer in Simulation, No.31, 1989, pp.189-194.

[152] S. J. Salon, M. J. DeBortoli and R. Palma, "Coupling Of Transient Fields, Circuits, And Motion Using Finite Element Analysis," J. Electromagnetic Waves \& Appl., Vol. 4, Nov, 1990, pp. 1077-1108.

[153] P. Zhou, W. N. Fu, D. Lin, S. Stanton and Z. J. Cendes, "Numerical Modeling of Magnetic Devices," IEEE Trans. on Magnetics, vol. 40, July 2004, pp. 18031809.

[154] P. Belforte, M. Chiampi, and M. Tartaglia, "A Finite Element Computation Procedure For electromagnetic fields under Different Supply Conditions," IEEE Trans. on Magnetics, vol.21, No.6, 1985, pp.2284-2287.

[155] H. Lindfors, and J. Luomi, "A General Method for the Numerical Solution of Coupled Magnetic Field and Circuit Equations," Proceedings of the International Conference on Electrical Machines, 1988, pp. 141-146.

[156] G. Bedrosian, "A New Method for Coupling Finite Element Field Solutions with External Circuits and Kinematics," IEEE Trans. on Magnetics, Vol. 29, March 1993, pp. 1664-1668.

[157] S. Kanerva, "Data Transfer Methodology between A FEA Program and a System Simulator," in Proceedings of IEEE-ICEMS Fifth Int. Conf., 2001, pp. 11211124.

[158] P. Eustache, G. Meunier, and J.L Coulomb, "Finite Element Toolbox for Generic Coupling," IEEE Trans. on Magnetics, Vol. 32, No. 3, May 1996, pp. 1461-1464.

[159] K. Hameyer, J. Driesen, H. De Gersem, and R. Belmans, "The Classification of Coupled Field Problems," IEEE Trans. on Magnetics, Vol. 35, No. 3, May 1999, pp.1618-1621.

[160] Shen, G. Meunier, J. L. Coulomb, and J. C. Sabonnadiere, "Solution of Magnetic Fields and Electrical Circuits Combined Problems," IEEE Trans. on Magnetics, Vol.MAG-21, No. 6, November 1985, pp. 2288-2291. 
[161] Vassent, G. Meunier, and A. Foggia, "Simulation of Induction Machines Using Complex Magnetodynamic Finite Element Method Coupled with the Circuit Equations," IEEE Trans. on Magnetics, Vol. 27, No. 5, September 1991, pp. 4246-4249.

[162] Vassent, G. Meunier, A. Foggia, and G. Reyne, "Simulation of Induction Machine Operation Using a Step by Step Finite Element Method Coupled with the Circuits and Mechanical Equations," IEEE Trans. on Magnetics, Vol. 27, No. 6, November 1991, pp.5232-5234.

[163] G. Strangas, and K. R. Theis, "Shaded Pole Motor Design and Evaluation Using Coupled Field and Circuit Equations," IEEE Trans. on Magnetics, Vol. MAG-21, No. 5, September 1985, pp. 1880-1882.

[164] E. G. Strangas, "Coupling the Circuit Equations to the Non-Linear Time Dependent Field Solution in Inverter Driven Induction Motors," IEEE Trans. on Magnetics, Vol.MAG-21, No. 6, November 1985, pp. 2408-2411.

[165] E. G. Strangas, and T. Ray, "Combining Field and Circuit Equations for the Analysis of Permanent Magnet AC Motor Drives," IEEE Industry Applications Society Annual Meeting, volume 1, Pittsburgh, USA, October 2-7, 1988, pp. 710.

[166] T. W. Preston, A.B.J Reece, and P.S Sangha, "Induction Motor Analysis by TimeStepping Techniques," IEEE Trans. on Magnetics, Vol. 24, No. 1, January 1988, pp.471-474.

[167] A. Arkkio, "Finite Element Analysis of Cage Induction Motors Fed by Static Frequency Converters," IEEE Trans. on Magnetics, Vol. 26, No. 2, March 1990, pp. 551-554.

[168] A Tsukerman, A. Konrad, and J. D. Lavers, "A Method for Circuit Connections in Time Dependent Eddy Current Problem," IEEE Trans. on Magnetics, Vol. 28, No. 2, March 1992, pp. 1701-1704.

[169] Piriou, and A. Razek, "Coupling of Saturated Electromagnetic Systems to NonLinear Power Electronic Devices," IEEE Trans. on Magnetics, Vol. 24, No. 1, January 1988, pp.274-277.

[170] F. Piriou, and A. Razek, "A Model for Coupled Magnetic-Electric Circuits in Electric Machines with Skewed Slots," IEEE Trans. on Magnetics, Vol. 26, No. 2, March 1990, pp. 1096-1100. 
[171] F. Piriou, and A. Razek, "Numerical Simulation of a Non-Conventional Alternator Connected to a Rectifier," IEEE Trans. on Energy Conversion, Vol. 5, No. 3, September 1990, pp. 512-518.

[172] N. Sadowski, B. Carly, Y. Lefevre, M. Lajoie-Mazenc, and S. Astier, "Finite Element Simulation of Electrical Motors Fed by Current Inverters," IEEE Trans. on Magnetics, Vol. 29, No. 2, March 1993, pp. 1683-1688.

[173] A. Demenko, "Time-Stepping FE Analysis of Electric Motor Drives with Semiconductor Converters," IEEE Trans. on Magnetics, Vol. 30, No. 5, September 1994, pp. 3264-3267.

[174] S. L Ho, W. N. Fu, H. L Li, H. C. Wong, and H. Tan, "Performance Analysis of Brushless DC Motors Including Features of the Control Loop in the Finite Element Modeling," IEEE Trans. on Magnetics, Vol. 37, No. 5, Sept. 2001, pp. $3370-3374$.

[175] M.A. Jabbar, H. N. Phyu, and Z. J. Liu, "Analysis of the Starting Process of a Disk Drive Spindle Motor by Time Stepping Finite Element Method," IEEE Trans. on Magnetics, Vol. 40, No. 4, July 2004, pp. 3204-3206.

[176] P. Kuo-Peng, J. P.A Bastos, N. Sadowski, and R. Carlson, "Analysis of a Combined Converter-Electromagnetic Device by Taking into Account its Control Loop," IEEE Trans. on Energy Conversion, Vol. 14, No. 4, December 1999, pp. $1430-1434$.

[177] L. Roel Ortiz, N. Sadowski, P. Kuo-Peng, N.J. Batistela, and J.P.A Bastos, "Coupling Static Converter With Control Loop and Non-Linear Electromagnetic Devices," IEEE Trans. on Magnetics, Vol. 37, No. 5, September 2001, pp. 3514 3517.

[178] T. Pham, P. Wendling, S. Salon, H. Acikgoz, “ Transient Finite Element Analysis of Induction Motor With External Circuit Connections And Electromechanical Coupling," IEEE Trans. on Energy Conversion, Vol. 14, No.4, Dec. 99, pp.14071411.

[179] G. Skibinski, R. Tallam, R. Reese, B. Buchholz, and R. Lukaszewski, "Common Mode and Differential Mode Analysis of Three Phase Cables for PWM AC Drives, " Proceedings of IEEE Industry Applications Conference Forty-First IAS Annual Meeting, Oct. 2006, pp. 880-888.

[180] Flux2D V9.2 User Manual, Magsoft Corporation 2003.

[181] Flux2D to Simulink Technology, Flux2D User Manual, Magsoft Corporation 2003. 
[182] D.J. Wilcox, M. Conlon, and W.G. Hurley, "Calculation of Self and Mutual Impedances for Coils on Ferromagnetic Core," IEE Proceedings, Vol. 135, Pt. A, No.7, Sept. 1988, pp. 470-476.

[183] D.J. Wilcox, W.G. Hurley and M. Conlon, "Calculation of Self and Mutual Impedances between Sections of Transformer Windings," IEE Proceeding, Vol. 136, Pt. C, No. 5, Sept. 1989, pp. 308-314.

[184] F. De Leon, and A. Semlyen, "Efficient Calculation of Elementary Parameters of Transformers," IEEE Trans. on Power Delivery, Vol.7, No. 1, Jan. 1992, pp.376383.

[185] R. Asensi, J. A. Cobos, O. Garcia, R. Prieto and J. Uceda, "A Full Procedure to Model High Frequency Transformer Windings," IEEE Power Electronics Specialists' Conference Proceedings, June1994, pp. 856-863.

[186] R. Prieto, R. Asensi, J. A. Cobos, O. Garcia, and J. Uceda, "Model of the capacitance effects in magnetic components," IEEE power electronics specialists' conference proceedings, June1995, pp. 678-683.

[187] V. A. Niemela, G. R. Skutt, A. M. Urling, Y. N. Chang et al., "Calculating the short circuit impedances of a multi winding transformer from its geometry," IEEE power electronics specialists' conference proceedings, 1989.

[188] J. Pleite, R. Prieto, R. Asensi, J. A. Cobos, and E. Olias, "Obtaining a frequency dependent and distributed effects model of magnetic components from actual measurements," IEEE Trans. on Magnetics, Vol. 35, No. 6, Nov. 1999, pp. 44904502.

[189] J. P. Vandelac and P. D. Ziogas, "A novel approach for minimizing high frequency transformer copper losses," IEEE Trans. on Power Electronics, vol.3, July 1988 , pp. 266-277.

[190] J.D. lavers and V Bolborici, "Loss comparison in the design of high frequency inductors and transformers," IEEE Trans. on Magnetics, vol. 35, Sept. 1999, pp. 3541-3543.

[191] F. Tourkhani and P. Viarouge, "Accurate analytical model of winding losses in round litz wire winding," IEEE Trans. on Magnetics, vol.37, Jan. 2001, pp.538543.

[192] A. Podoltsev, I. N. Kucheryavaya, and B. Lebedev, "Analysis of effective resistance and eddy current losses in multiturn winding of high frequency magnetic components," IEEE Trans. on Magnetics, Vol.39, No.1, Jan. 2003, pp. 539-548. 
[193] S. Mallat, "A Wavelet Tour of Signal Processing," Academic Press; 2nd edition, 1999.

[194] I. Daubechies," Ten Lectures on Wavelets," SIAM: Society for Industrial and Applied Mathematics, 1992.

[195] G. String and T. Nguyen, "Wavelets and Filter Banks," Cambridge, MA: Wellesley-Cambridge Press, 1996.

[196] V. K. Madisetti, and D. B. Williams, "Digital Signal Processing Handbook," CRC Press, 1999.

[197] O. A. Mohammed, S. Ganu, N. Abed, S. Liu and Z. Liu, "High Frequency PM Synchronous Motor Model Determined by FE Analysis," IEEE Trans. on Magnetics, Vol. 42, April 2006, pp. 1291-1294.

[198] O. A. Mohammed, S. Ganu, N. Abed, S. Liu and Z. Liu, "High Frequency PM Synchronous Motor Model Determined by FE Analysis," Compumag 2005.

[199] O. A. Mohammed, S. Ganu, S. Liu, Z. Liu and N. Abed, "Study of High Frequency Model of Permanent Magnet Motor," IEMDC 2005.

[200] O. A. Mohammed, S. Ganu, N. Abed, S. Liu, Z. Liu, "FE-based Physical Phase Variable Model of the RSPS Motor for Integrated Drive Applications," EMTS 2006.

[201] O. A. Mohammed, S. Ganu, N. Abed, S. Liu, Z. Liu, "High Frequency Phase Variable Model of Electric Machines from Electromagnetic Field Computation," ACES 2006.

[202] O. A. Mohammed, S. Ganu, N. Abed, Z. Liu and S. Liu, "High Frequency Phase Variable Model of Electric Machines for the Simulation of Drive Interactions," IGTE 2006.

[203] Y. Yin, and H. W. Dommel, "Calculation of frequency-dependent impedances of underground power cables with finite element method," IEEE Trans. on Magnetics, Vol. 25, No.4, July 1989, pp. 3025-3027.

[204] L. Bobrow, "Elementary Linear Circuit Analysis," pp.575-577.

[205] A. Lima, H. Dommel, and R. Stephan, "Modeling Adjustable Speed Drives With Long Feeders," IEEE Trans. on Industrial Electronics, Vol.47, No.3, June 2000, pp.549-556. 
[206] O. V. Thorsen, and M. Dalva, "Modeling Of Converter-Fed Motor Drives For Analyzing Motor Transients," IEEE Conference, 2001, pp.1139-1146.

[207] C. Dufour, S. Abourida, and J. Bélanger, "Hardware-In-the-Loop Simulation of Power Drives with RT-LAB," Opal-RT Technologies, Inc, White Paper.

[208] S. Abourida, C. Dufour, and J. Bélanger, "Real-Time HIL Simulation of a Complete PMSM Drive at $10 \mu$ s Time Step," Opal-RT Technologies, Inc, White Paper.

[209] S. Abourida, C. Dufour, and J. Bélanger, "Real-Time and Hardware-In-The-Loop Simulation of Electric Drives and Power Electronics: Process, problems and solutions," International Power Electronics Conference, 2005.

[210] S. Abourida, C. Dufour, and J. Bélanger, "Hardware-In-the-Loop Simulation of Finite-Element Based Motor Drives with RT-LAB and JMAG," Opal-RT Technologies, Inc, White Paper.

[211] C. Dufour, "FPGA-Based Real-Time Simulation of Permanent Magnet Synchronous Motor Drive for Vehicular Applications," Opal-RT Technologies, Inc, White Paper.

[212] S. Abourida, C. Dufour, J. Belanger, and V. Lapointe, "Real time, PC- Based Simulator of Electric Systems and Drives," International Conference On Power System Transients 2003.

[213] RT-Lab, version 8.2 User Guide.

[214] RT-Events, Version 2.2 User Guide

[215] Ned Mohan, "Electric Drives- An Integrative Approach," MNPERE, 2001.

[216] Ned Mohan, "Advanced Electric Drives: Analysis, Control and Modeling Using Simulink," MNPERE, 2001.

[217] N. Mohan, W.P. Robbins, P. Imbertson, T.M. Undeland, R.C. Panaitescu, A.K. Jain, P. Jose, and T. Begalke, "Restructuring of First Courses in Power Electronics and Electric Drives That Integrates Digital Control," IEEE Trans. on Power Electronics, Vol. 18, Issue 1, Part 2, Jan. 2003, pp.429- 437.

[218] N. Mohan, "DSP Based Electric Drives Laboratory," User manual, University of Minnesota, July 2004.

[219] Wavelet Toolbox User's Guide, Mathworks, Version 2, 2002. 
[220] B. Bose, "Modern Power Electronics and AC Drives," Prentice Hall 2002.

[221] O. A. Mohammed, S. Ganu, N. Abed, Z. Liu, S. Liu, "High Frequency Modeling of Permanent Magnet Synchronous Motor Drive," IEMDC 2007, pp. .

[222] O. A. Mohammed, S. Ganu, Z. Liu, N. Abed, S. Liu, "Conducted Electromagnetic Interference Prediction in Integrated Motor Drive," ACES 2007.

[223] S. Abourida, C. Dufour, J. Belanger, V. Lapointe, "Real time, PC- Based Simulator of Electric Systems and Drives," International conference on power system transients 2003.

[224] P.S. Shiakolas, and D. Piyabongkarn, "Development of a Real-Time Digital Control System With a Hardware-in-the-Loop Magnetic Levitation Device for Reinforcement of Controls Education," IEEE Trans. on education IEEE Trans, vol. 46, Feb. 2003, pp. 79-87.

[225] O. Mohammed, and R. Sebastien "A Real-time Electromagnetic Analysis of Electric Machines for Educational Purposes and Laboratory Implementation," IEEE Trans. on Magnetics, vol.34, No. 5, Sept. 1998, pp. 3628-3631. 


\section{VITA}

\section{NAGY YOUSSEF ABED}

1973

Born, Mansoura, Dakahlia, Egypt

$1991-1996$

Bachelor of Engineering in Electrical Engineering, Mansoura University, Egypt. First Rank in the class.

1996-1999

M.S. in Electrical Engineering, Mansoura University, Mansoura, DK, Egypt

1996-2003

Research Assistance, Mansoura University, Egypt

2003-2007

Doctoral candidate. in Electrical Engineering, Florida International University, Miami, Florida, USA

\section{SELECTED PUBLICATIONS}

1. N.Y. Abed, and O. A. Mohammed, "Modeling and Characterization of Transformers Internal Faults using Finite Element and Discrete Wavelet Transforms", IEEE Transactions on Magnetics, Volume 43, Issue 4, April 2007, pp. 1425-1428.

2. O. A. Mohammed, N.Y. Abed, and S. Liu "Investigation of the Harmonic Behavior of Three Phase Transformer Under Nonsinusoidal Operation Using Finite Element and Wavelet Packets", IEEE Transactions on Magnetics, Volume 42, Issue 4, April 2006, pp. 967-970.

3. O. A. Mohammed, N.Y. Abed, and S. Ganu "Modeling and characterization of induction motor internal faults using finite element and discrete wavelet transforms", IEEE Transactions on Magnetics, Volume 42, Issue 4, Oct. 2006, pp. $3434-3436$.

4. O. A. Mohammed, Z. Liu, S. Liu, and N.Y. Abed "Internal Short Circuit Fault Diagnosis for PM Machines Using FE-based Phase Variable Model and Wavelet Analysis", IEEE Transactions on Magnetics, Vol. 3, NO.4, April 2007, pp.17291732.

5. O. A. Mohammed, Liu, Z., S. Liu, and N.Y. Abed, "Finite-Element-Based Nonlinear Physical Model of Iron-Core Transformers for Dynamic Simulations" IEEE Transactions on Magnetics, Volume 42, Issue 10, April 2006, pp.1027-1030. 
6. O. A. Mohammed, S. Ganu, N.Y. Abed, S. Liu and Z. Liu, "High Frequency PM Synchronous Motor Model Determined by FE Analysis," IEEE transactions on Magnetics, April 2006, pp.1291-1294..

7. O. A Mohammed, Z Liu, S. Liu, and N.Y. Abed "Electric machine operational modeling utilizing coupled electromagnetic and electric drives simulations," International Journal for Computation and Mathematics in Electrical Engineering (COMPEL), Volume24 (2), pp. 495-508.

8. O. A. Mohammed, N.Y. Abed, and S. Ganu "Modeling and characterization of induction motor internal faults using finite element and discrete wavelet transforms", INTERMAG Conference, 2006

9. O. A. Mohammed, N. Y. Abed "Modeling and Characterization of Transformers Internal Faults using Finite Element and Discrete Wavelet Transforms" CEFC Conference, Miami 2006.

10. O. A. Mohammed, N. Y. Abed, and S. Ganu, "Application of Finite element to High Frequency transformer modeling”, CEFC Conference, Miami 2006.

11. O. A. Mohammed, N. Y. Abed, and S. Liu, "Coupled Physical finite element analysis of distribution Transformer for on-line Fault studies" CEFC Conference, Seoul 2004.

12. O. A. Mohammed, N. Y. Abed, and S. Ganu, "Real-Time Simulations of Electrical Machine Drives with Hardware-in-the-Loop", IEEE PES General Meeting 2007.

13. O. A. Mohammed, S. Ganu, S. Liu, Z. Liu and N.Y. Abed, "Study of High Frequency Model of Permanent Magnet Motor," International electric machines and drives conference, IEMDC 2005.

14. O.A. Mohammed, N.Y. Abed, S. Ganu, and Shuo Liu, " Acoustic noise signal evaluation due to magnetostrictive effects in electrical equipment" IEEE/ACES International Conference on Wireless Communications and Applied Computational Electromagnetics, 2005.

15. O. A. Mohammed, S. Ganu, N.Y. Abed, S. Liu, Z. Liu, "High Frequency Phase Variable Model of Electric Machines from Electromagnetic Field Computation," Applied computational electromagnetics society, ACES 2006.

16. O. A. Mohammed, S. Liu, Z. Liu, N.Y. Abed, and S. Ganu, "Innovations in Teaching Energy Systems utilizing an Integrated Simulation with Hardware in the Loop," IEEE Power Engineering Society General Meeting, PES 2005. 